\title{
MULTI-SCALE FAILURE ANALYSIS OF LAMINATED COMPOSITES USING THE BOUNDARY ELEMENT METHOD
}

\author{
By
}

\section{Rene Quispe Rodríguez}

A thesis submitted to the Faculty of Graduate and Posdoctoral Affairs in partial fulfillment of the requirements for the degree of Doctor of Philosophy

Ottawa-Carleton Institute for Mechanical and Aerospace Engineering Department of Mechanical and Aerospace Engineering Carleton University Ottawa, Ontario

March, 2016

CCopyright

2016, Rene Quispe Rodríguez 


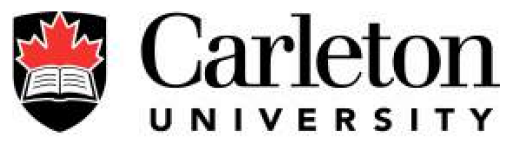

\title{
Falha multiescala de compósitos laminados usando o método dos elementos de contorno
}

\author{
Multi-scale failure analysis of laminated \\ composites using the boundary element method
}




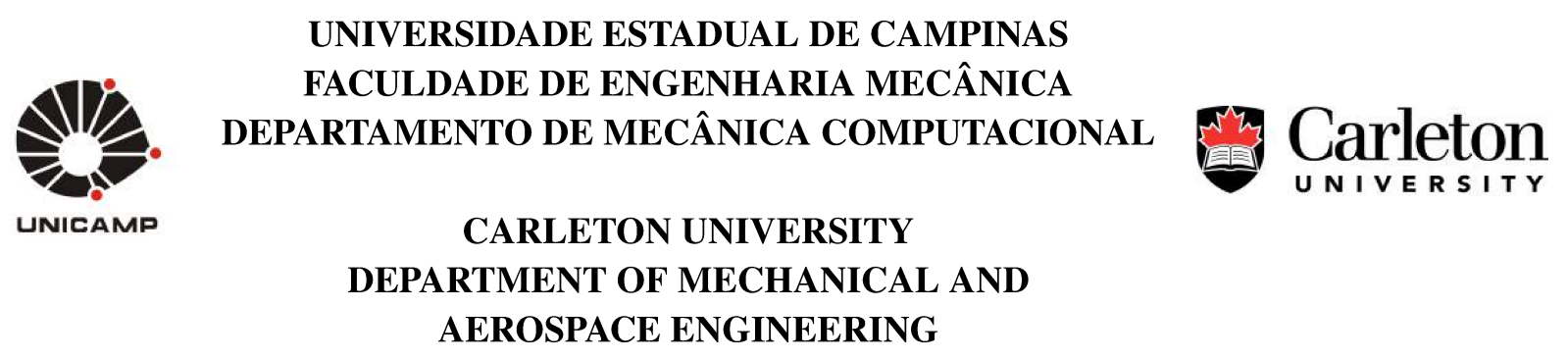

Rene Quispe Rodríguez

Falha multiescala de compósitos laminados usando o método dos elementos de contorno

Tese apresentada, mediante um acordo de Cotutela, (a) à Faculdade de Engenharia Mecânica da Universidade Estadual de Campinas (UNICAMP), Brasil, e (b) à Faculdade de Pós-Graduação e Assuntos de Pós-Doutorado, Carleton University, Canadá, como parte dos requisitos exigidos para obtenção do título de Doutor em Engenharia Mecânica, na Área de Mecânica dos Sólidos e Projeto Mecânico da UNICAMP, e para a obtenção do título de Doutor em Filosofia (Ph.D.) em Engenharia Mecânica da Carleton University.

Orientador UNICAMP: Prof. Dr. Paulo Sollero

Orientador Carleton University: Prof. Dr. Choon-Lai Tan

Co-orientador UnB: Prof. Dr. Éder Lima de Albuquerque

ESTE EXEMPLAR CORRESPONDE À VERSÃO FINAL DA TESE DEFENDIDA PELO ALUNO RENE QUISPE RODRÍGUEZ E ORIENTADA PELO PROF. DR. PAULO SOLLERO E PELO PROF. DR. CHOONLAI TAN.

Prof. Dr. Paulo Sollero

Prof. Dr. Choon-Lai Tan

CAMPINAS

2016 


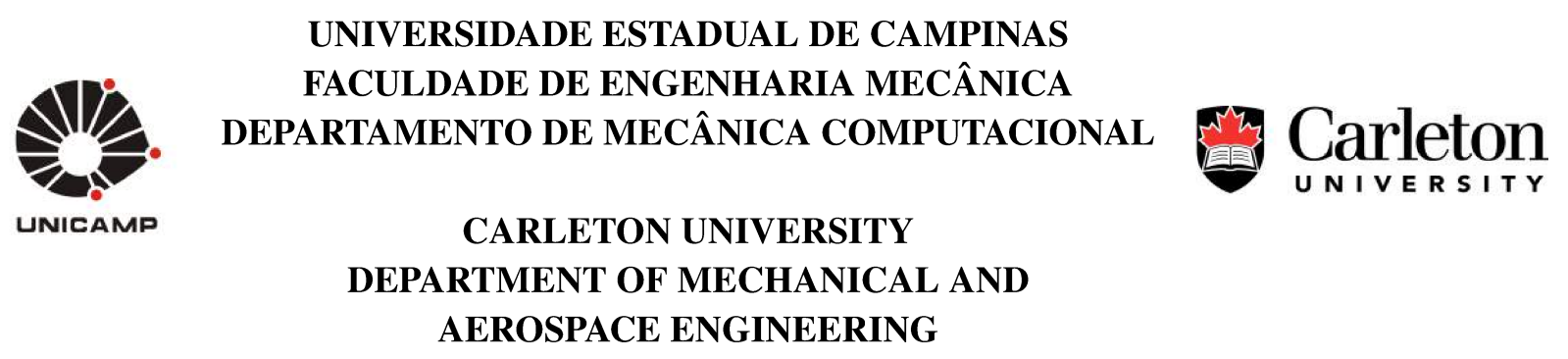

Rene Quispe Rodríguez

Multi-scale failure analysis of laminated composites using the boundary element method

\begin{abstract}
Thesis presented, under a Cotutelle Agreement, to (a) the School of Mechanical Engineering, University of Campinas (UNICAMP), Brazil, and (b) Faculty of Graduate and Posdoctoral Affairs, Carleton University, Canada, in partial fulfilment of the requirements for the degree of Doctor in Mechanical Engineering, in the Area of Solid Mechanics and Mechanical Design at UNICAMP, and the degree of Doctor of Philosophy (Ph.D.) in Mechanical Engineering at Carleton University.
\end{abstract}

Advisor UNICAMP: Prof. Dr. Paulo Sollero

Advisor Carleton University: Prof. Dr. Choon-Lai Tan

Co-advisor UnB: Prof. Dr. Éder Lima de Albuquerque

THIS COPY CORRESPONDS TO THE FINAL VERSION OF THE THESIS DEFENDED BY THE STUDENT RENE QUISPE RODRÍGUEZ AND ADVISED BY PROF. DR. PAULO SOLLERO AND BY PROF. DR. CHOON-LAI TAN.

Prof. Dr. Paulo Sollero

Prof. Dr. Choon-Lai Tan

CAMPINAS

2016 
Agência(s) de fomento e $\mathbf{n}^{\circ}$ (s) de processo(s): FAPESP, 2012/24297-0; CNPq, 148688/2011-0

Ficha catalográfica

Universidade Estadual de Campinas

Biblioteca da Área de Engenharia e Arquitetura

Elizangela Aparecida dos Santos Souza - CRB 8/8098

Quispe Rodríguez, Rene, 1987-
Q487m Multi-scale failure analysis of laminated composites using the boundary

element method / Rene Quispe Rodríguez. - Campinas, SP : [s.n.], 2016.

Orientadores: Paulo Sollero e Choon-Lai Tan.

Coorientador: Éder Lima de Albuquerque.

Tese (doutorado) - Universidade Estadual de Campinas, Faculdade de Engenharia Mecânica.

Em cotutela com Carleton University

1. Falhas de sistemas. 2. Compósitos. 3. Multiescala. 4. Métodos de elementos de contorno. 5. Aceleração (Mecânica). I. Sollero, Paulo,1950-. II. Tan, Choon-Lai. III. Albuquerque, Éder Lima,1972-. IV. Universidade Estadual de Campinas. Faculdade de Engenharia Mecânica. V. Carleton University. VI. Título.

\section{$\underline{\text { Informações para Biblioteca Digital }}$}

Título em outro idioma: Falha multiescala de compósitos laminados usando o método dos elementos de contorno

Palavras-chave em inglês:

System failures

Composites

Multiscale modeling

Boundary elements method

Acceleration (Mechanical)

Área de concentração: Mecânica dos Sólidos e Projeto Mecânico

Titulação: Doutor em Engenharia Mecânica

Banca examinadora:

Paulo Sollero [Orientador]

Choon-Lai Tan [Orientador]

Euclides de Mesquita Neto

Sergio Franscino Muller de Almeida

Xin Wang

Data de defesa: 31-03-2016

Programa de Pós-Graduação: Engenharia Mecânica 
UNIVERSIDADE ESTADUAL DE CAMPINAS

FACULDADE DE ENGENHARIA MECÂNICA

DEPARTAMENTO DE MECÂNICA COMPUTACIONAL

CARLETON UNIVERSITY

DEPARTMENT OF MECHANICAL AND AEROSPACE

ENGINEERING

Ph.D. THESIS

\section{Multi-scale failure analysis of laminated composites using the boundary element method}

Falha multiescala de compósitos laminados usando o método dos elementos de contorno

Author: Rene Quispe Rodríguez

Advisor UNICAMP: Prof. Dr. Paulo Sollero

Advisor Carleton University: Prof. Dr. Choon-Lai Tan

Co-advisor UnB: Prof. Dr. Éder Lima de Albuquerque

The examiners bench composed by the following members approved this thesis:

Prof. Dr. Paulo Sollero

DMC/FEM/UNICAMP

Prof. Dr. Choon-Lai Tan

M\&AE/Carleton University

Prof. Dr. Euclides de Mesquita Neto

DMC/FEM/UNICAMP

Prof. Dr. Sergio Franscino Muller de Almeida

IEM/ITA

Prof. Dr. Xin Wang

M\&AE/Carleton University 


\section{Acknowledgements}

To my dear son, René Junior Quispe Couto, for giving me happiness during the last two years of my studies and for being the ultimate reason for finishing this work.

To my family for their love and affection.

To my friends.

To my PhD advisors: Professor Paulo Sollero and Professor Choon-Lai Tan, for their unwavering support, friendship and dedication throughout this work.

To my PhD co-advisor, Professor Eder Lima de Albuquerque, for all discussions, friendship and dedication throughout this work

To University of Campinas for the opportunity to carry out this work.

To Carleton University for providing the academic environment and support during the one-year period of the project.

To University of Brasilia for the academic environment and support during some stages of the project.

To CNPq for the financial support.

To FAPESP for the financial support.

To CENAPAD/UNICAMP for the support of computational facilities.

To the Center for Computational Engineering and Science - CCES/UNICAMP for the support and use of the computational facilities. 
"My mission in life is not merely to survive, but to thrive; and to do so with some passion, some compassion, some humor, and some style."

M. Angelou 


\section{Resumo}

RODRÍGUEZ, René. Falha multiescala de compósitos laminados usando o método dos elementos de contorno. 2015. XXXp. Tese (Doutorado). Faculdade de Engenharia Mecânica, Universidade Estadual de Campinas, Campinas, Brasil.

O projeto tem como objetivo principal a construção de ferramentas computacionais que sejam capazes de facilitar a modelagem multiescala de falhas em compósitos laminados. Primeiro, para a modelagem na meso-escala, o método dos elementos de contorno (BEM) é usado, onde a solução fundamental anisotrópica 3D baseada em séries duplas de Fourier foi aplicada. A vantagem mais significativa do uso da representação de séries de Fourier e as suas derivadas é que os coeficientes de Fourier precisam ser calculados uma única vez independentemente do número de pontos campo. Este fato reduz os esforços computacionais, além de, facilitar a implementação em um código do BEM. Os efeitos dinâmicos no meio contínuo foram incluídos na equação de equilíbrio. Estes efeitos induziram integrais de domínio na equação integral de contorno (BIE). O Método de Dupla Reciprocidade (DRM) e o Método de Integração Radial (RIM) foram implementados para a transformação destas integrais de domínio em integrais de contorno. Adicionalmente, foram usadas técnicas de aceleração do BEM, tais como a Aproximação Cruzada Adaptativa (ACA). A combinação da solução anisotrópica 3D e o ACA permitiram um ganho substancial na rapidez de processamento, assim como na armazenagem de memória, fato importante quando são analisados problemas de grande porte como é um problema multiescala. A seguir, o critério multiescala foi implementado e aplicado a compósitos laminados. Na análise micro-escala, um potencial específico para materiais epoxi foi usado. A seguir, a regra de Cauchy-Born foi usada para acoplar as escalas no modelo multiescala 3D. Alguns exemplos numéricos foram apresentados para mostrar a validade do critério de falha. O projeto contemplou um período de um ano na Carleton University em Ottawa, Canada, sob a supervisão do Professor Choon-Lai Tan, especialista em mecânica da fratura, formulação de materiais anisotrópicos e elementos de contorno. Este período no exterior permitiu ao aluno uma formação acadêmica de boa qualidade, assim como permitirá a obtenção de um duplo-diploma. Mediante um termo de co-tutela o estudante, quando aprovado, será reconhecido com o grau de doutor na Carleton University e na UNICAMP.

Palavras-chave: Critérios de falha; Compósitos; Multiescala; Método dos elementos de contorno; Métodos de aceleração 


\begin{abstract}
RODRÍGUEZ, René. Multiscale failure analysis of laminated composites using the boundary element method. 2015. XXXp. Thesis (PhD). School of Mechanical Engineering, University of Campinas, Campinas, Brazil.

This project has as its main objective the development of the computational tools capable of facilitating multi-scale failure analysis in laminated composites. First, for the continuum meso-scale, the boundary element method (BEM) is used, in which the anisotropic 3D fundamental solutions based on double Fourier series was employed. The most significant advantage of using this Fourier series representation and its derivatives is that Fourier coefficients need to be evaluated just once independent of the number of field points. This fact reduced the computational efforts, besides facilitating the ease of implementation into a BEM code. The dynamic effects in the continuum media were included in the equilibrium equation. These effects induced domain integrals in the boundary integral equation (BIE). The Dual Reciprocity Method (DRM) and the Radial Integration Method (RIM) were implemented for the transformation of the domain integrals into boundary integrals. Additionally, an acceleration technique namely the Adaptive Cross Approximation (ACA) was used with the BEM. The combination of the 3D anisotropic fundamental solutions with ACA resulted in a substantial memory and processing speed gain, that is of main importance when huge numerical problems, such as multi-scale problems, are being analized. Next, the failure multi-scale criterion was implemented and applied to laminated composites. For the micro-scale analysis, a specific potential for epoxy materials was used. Then, the Cauchy-Born rule was used to couple the scales on the 3D multi-scale model. Some numerical examples were presented in order to show the validity of the failure criterion. The project involved a one-year period at Carleton University in Ottawa, Canada, under the supervision of Prof. Dr. Choon-Lai Tan, specialist in fracture mechanics, anisotropic material formulation and boundary elements. This period in Canada provided the student with a strong academic formation, besides, providing the possibility of obtaining a double diploma. Through a cotutelle agreement, the student will receive a Ph.D. degree from Carleton University as well as from UNICAMP.
\end{abstract}

Keywords: Failure criteria; Composites; Multi-scale; Boundary element method; Acceleration methods 


\section{List of Figures}

1.1 Fragment of polymer (oligomer) modeled by MD; (a) Polyethylene (PE); (b) Polypropylene (PP); (c) Polyacrylic acid (PAA); (d) Polurea (PU), (YANOVSKY, 2005).

1.2 Comparison of real fiber arrangement and idealized fiber arrays; (a) Fiber array in real composite; (b) square fiber array; (c) hexagonal fiber array; (d) diamond fiber array, (JIN et al., 2008).

1.3 An engine block discretized using FEM and BEM; (a) FEM (363,000 volume elements/1.5 million DOFs); (b) BEM (42,000 surface elements/DOFs),

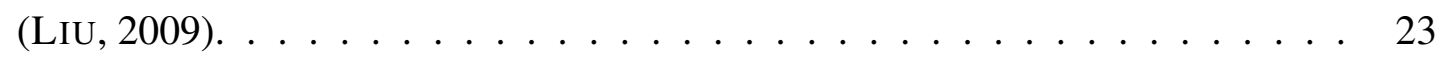

2.1 Lamina principal axes $\left(x_{1}\right.$ and $\left.x_{2}\right)$ and laminate reference axes $\left(\bar{x}_{1}\right.$ and $\left.\bar{x}_{2}\right) \ldots \quad \ldots 38$

2.2 (a) Shaking-Geometry Composite, (b) deviation of the fibers about the regular square lattice. . . . . . . . . . . . . . . . . . 41

3.1 Normal vectors at the field point $Q \ldots \ldots \ldots \ldots$. . . . . . . 44

3.2 (a) Eight-node discontinuous quadratic element. . . . . . . . . . . . . . 54

3.3 Multi-domain 2D example with two sub-regions. . . . . . . . . . . 56

3.4 Multi-domain example with four fibers. . . . . . . . . . . . . 58

3.5 (a) Equivalent beam model; (b) BEM mesh (Symmetry). . . . . . . . . . . . . 64

3.6 Normalized transverse displacements. . . . . . . . . . . . . . . 65

3.7 Normalized direct stresses at $x_{1}=2 H, 3 H, 4 H, 5 H \ldots \ldots \ldots \ldots 6$

3.8 (a) geometry of Example C; (b) BEM mesh. . . . . . . . . . . . . . . . 67

3.9 Comparison of the $u_{1}$ displacement around the circumferential hole in the mid-

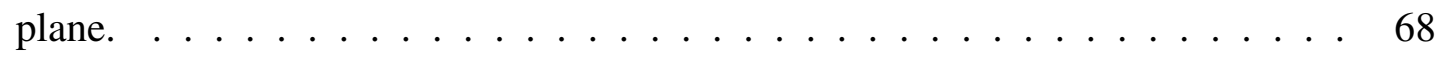

3.10 (a) geometry of example 3; (b) BEM mesh. . . . . . . . . . . . . . . . 69

3.11 Normalized total displacement comparison. . . . . . . . . . . . . . 70

3.12 (a) Geometry and boundary conditions for example A; (b) BEM mesh. . . . . . 71

3.13 Normalized transverse displacements. . . . . . . . . . . . . . 72

3.14 Normalized normal stresses at $x_{1}=4 H, 6 H, 8 H \ldots \ldots \ldots 72$

3.15 Normal displacement comparison. . . . . . . . . . . . . . . 73

4.1 Cell domain discretization with integration cells $\Omega_{k}$ in a $2 \mathrm{D}$ problem. . . . . . 76

4.2 General 3D domain differential. . . . . . . . . . . . . . . 79

4.3 Bar stretched by its own weight. . . . . . . . . . . . . 85

$4.4 u_{3}$ displacements for a bar stretched by its own weight. . . . . . . . . . 86

4.5 Transverse displacement comparison. . . . . . . . . . . . . . 86 
4.6 Displacement in $x . \quad \ldots \ldots \ldots \ldots$

$4.7 u$ displacement along the $x$ axis. . . . . . . . . . . . . . . . 90

$4.8 v$ displacement along the $y$ axis. . . . . . . . . . . . . . . 90

$4.9 w$ displacement along the $z$ axis. . . . . . . . . . . . . . . 91

4.10 Geometry, boundary conditions and mesh of the isotropic bar. . . . . . . . . . 92

4.11 Mesh for the RIM analysis. . . . . . . . . . . . . . . . . . . . 93

$4.12 u_{1}(t)$ displacement comparison at $(50,5,5) \mathrm{mm} \ldots \ldots \ldots$. . . . . . . . . 94

4.13 Geometry, boundary conditions and mesh of the isotropic bar. . . . . . . . . . 95

$4.14 u_{1}(t)$ displacement comparison at $(50,5,5) \mathrm{mm} \ldots \ldots \ldots \ldots$

5.1 Clusters created in the first two levels (KuRZ et al., 2007) . . . . . . . . . . . 98

5.2 Admissible cluster pair (KuRz et al., 2007) . . . . . . . . . . . . . . 99

5.3 Example for the application of ACA in multidomain problems. . . . . . . . . 103

5.4 (a) Collocation matrix for the four inclusion example, (b) contributions for physical nodes for the inclusion example . . . . . . . . . . . . . . . . . . . . 104

5.5 Geometry of the bar showing the elements. . . . . . . . . . . . . . 105

5.6 Cluster division in a tree format, better known as cluster tree. . . . . . . . . 106

5.7 Cluster division - geometrical display. . . . . . . . . . . . . . . 107

5.8 Block generation. . . . . . . . . . . . . . . . . . . . . 108

5.9 Illustrative figure showing the regular lattice (a), and the shaking-geometry configuration (b). . . . . . . . . . . . . . . . . . . . . 109

5.10 Block-wise representation of the collocation matrix for different admissibility parameters $(\eta$ ): (a) $\eta=0.6$, (b) $\eta=0.8$, (c) $\eta=1.0$, (d) $\eta=\sqrt{2.0}$ and (e)

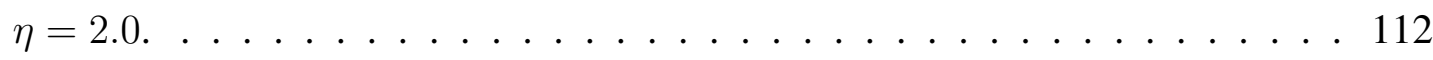

5.11 (a) Speed-up ratio as a function of the number of elements, (b) Speed-up ratio as a function of $\eta \ldots \ldots \ldots$. . . . . . . . . . . . . . . . 113

5.12 (a) BEM coarsest mesh; (b) BEM most refined mesh. . . . . . . . . . . . . . 115

5.13 BEM and ACA cpu times. . . . . . . . . . . . . . . 116

5.14 (a) BEM most refined mesh; (b) BEM coarsest mesh. . . . . . . . . . . . . . . 117

5.15 Conventional BEM and ACA cpu times. . . . . . . . . . . . . . 118

5.16 (a) Fiber-Matrix model with 36 fibers; (b) unit cell with a randomly oriented short fiber. . . . . . . . . . . . . . . . . . . . . . . . . . 119

5.17 Fiber-matrix model with an isotropic material. . . . . . . . . . . . . . 120

5.18 Numerical comparison with the analytical solution. . . . . . . . . . . 120

5.19 Displacement on the $x_{3}$ direction for the most refined case. . . . . . . . . . . 122

$5.20 \mathrm{Cpu}$ times comparison between conventional BEM and ACA. . . . . . . . . 123

6.1 Epoxy compound represented as short chains of beads (YANG et al., 2014). . 125

6.2 Bond potential curves. . . . . . . . . . . . . . . . 126 
6.3 (a) Integration over half-space, (b) Molecule-body interaction in plane xz. . . . 127

6.4 Bond potential curves. . . . . . . . . . . . . . . . . . . . 128

6.5 Derivative of the bond potential curves. . . . . . . . . . . . . . . . . 129

6.6 Nodal displacements for a interface 3D element. . . . . . . . . . . . . . . . 131

6.7 Multi-scale analysis flowchart. . . . . . . . . . . . . . . 135

6.8 Cohesive traction forces components. . . . . . . . . . . . . . . 136

6.9 Cohesive normal force, when the imposed displacement is in the $X$ coordinate axis. . . . . . . . . . . . . . . . . . . 137

6.10 Cohesive normal force, when the imposed displacement is in the $Y$ coordinate axis. . . . . . . . . . . . . . . . . . . . . . 138

6.11 Cohesive normal force, when the imposed displacement is in the $Z$ coordinate axis.

6.12 (a) Laminate composite model with a $0^{\circ} / 90^{\circ}$ distribution and 4 fibers per lamina;

(b) Discretization of the interface at the laminate composite. . . . . . . . . . . 141

6.13 Boundary conditions of the composite laminate . . . . . . . . . . . . . . 142

6.14 (a) Laminate implementation issue due to discretization, (b) Laminate interface. 143

6.15 Laminate composite with a $0^{\circ} / 0^{\circ}$ fiber distribution; (a) Boundary conditions, (b) Fiber discretization and initial failure (red). . . . . . . . . . . . . . . . . . 144

6.16 Multi-scale failure: (a) Time step 1, $P=1400 \mathrm{MPa}, t=0 \mathrm{~ms}$; (b) Time step 50, $P=2665 \mathrm{MPa}, t=24 \mathrm{~ms}$; (c) Time step 70, $P=3181 \mathrm{MPa}, t=34.5 \mathrm{~ms} \ldots \ldots$

6.17 Multi-scale failure: (a) Time step 100, $P=3955 \mathrm{MPa}, t=49.5 \mathrm{~ms}$; (b) Time step $111, P=4239 \mathrm{MPa}, t=55 \mathrm{~ms}$; (c) Time step 128, $P=4677 \mathrm{MPa}, t=$ $63.5 \mathrm{~ms}$

6.18 Multi-scale failure: (a) Time step 148, $P=5194 \mathrm{MPa}, t=73.5 \mathrm{~ms}$; (b) Time step $164, P=5607 \mathrm{MPa}, t=81.5 \mathrm{~ms}$; (c) Time step $180, P=6019 \mathrm{MPa}$, $t=89.5 \mathrm{~ms}$

6.19 Short-fiber composite example; (a) Boundary conditions, (b) Fiber discretization and initial failure (red).

6.20 Multi-scale failure: (a) Time step 1, $P=3400 \mathrm{MPa}, t=0 \mathrm{~ms}$; (b) Time step 25, $P=4636 \mathrm{MPa}, t=12 \mathrm{~ms}$; (c) Time step 50, $P=5924 \mathrm{MPa}, t=24.5 \mathrm{~ms}$

6.21 Multi-scale failure: (a) Time step 70, $P=6954 \mathrm{MPa}, t=34.5 \mathrm{~ms}$; (b) Time step $71, P=7006 \mathrm{MPa}, t=35 \mathrm{~ms}$; (c) Time step 72, $P=7057 \mathrm{MPa}, t=35.5 \mathrm{~ms} \quad . \quad 152$

6.22 Multi-scale failure: (a) Time step 73, $P=7109 \mathrm{MPa}, t=36 \mathrm{~ms}$; (b) Time step 74, $P=7160 \mathrm{MPa}, t=36.5 \mathrm{~ms}$; (c) Time step 75, $P=7212 \mathrm{MPa}, t=37 \mathrm{~ms} \quad$.

6.23 Laminate composite with a $0^{\circ} / 0^{\circ}$ fiber distribution; (a) Boundary conditions, (b) Fiber discretization and initial failure (red). 
6.24 Multi-scale failure: (a) Time step 1, $P=8100 \mathrm{MPa}, t=0 \mathrm{~ms}$; (b) Time step 25, $P=9409 \mathrm{MPa}, t=12 \mathrm{~ms}$; (c) Time step 50, $P=10773 \mathrm{MPa}, t=24.5 \mathrm{~ms} \quad \ldots 156$

6.25 Multi-scale failure: (a) Time step 58, $P=11209 \mathrm{MPa}, t=28.5 \mathrm{~ms}$; (b) Time step 59, $P=11264 \mathrm{MPa}, t=29 \mathrm{~ms}$; (c) Time step 60, $P=11318 \mathrm{MPa}, t=$

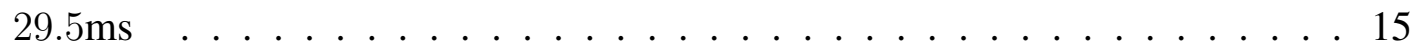

6.26 Multi-scale failure: (a) Time step 61, $P=11373 \mathrm{MPa}, t=30 \mathrm{~ms}$; (b) Time step 62, $P=11427 \mathrm{MPa}, t=30.5 \mathrm{~ms}$; (c) Time step 63, $P=11482 \mathrm{MPa}, t=31 \mathrm{~ms}$ 


\section{List of Tables}

3.1 Computed values of the Green's function $U_{i j}$ at $(r=1.0, \theta=\pi / 3, \phi=\pi / 4) . \quad$. 61

3.2 Computed first order derivative, $U_{i j, l}$, at $(r=1.0, \theta=\pi / 3, \phi=\pi / 4) . \ldots 62$

3.3 Computed second order derivatives, $U_{i j, l k}$, at $(r=1.0, \theta=\pi / 3, \phi=\pi / 4) \ldots \quad \ldots 3$

4.1 Displacements $u_{3}$ for DRM, RIM and comparison to the analytical solution. . . 87

5.1 CPU time ratios for each case . . . . . . . . . . . . . . . . 110

5.2 Computed effective conductivity for each case . . . . . . . . . . . . 114

5.3 Coordinates of the random nodes. . . . . . . . . . . . . . . . . 122

5.4 Nodal comparison between conventional BEM and ACA for random nodes. . . 123 


\section{TABLE OF CONTENTS}

$\begin{array}{ll}\text { List of Figures } & 11\end{array}$

$\begin{array}{ll}\text { List of Tables } & 15\end{array}$

TABLE OF CONTENTS

1 Introduction $\quad 18$

1.1 Motivation . . . . . . . . . . . . . . . . . 26

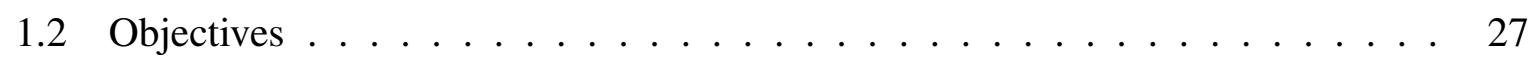

1.3 Contributions . . . . . . . . . . . . . . . 27

1.4 Thesis organization $\ldots \ldots \ldots \ldots \ldots \ldots$

2 Constitutive equations and equilibrium for anisotropic materials 31

2.1 Introduction . . . . . . . . . . . . . . . 31

2.2 Anisotropic elasticity . . . . . . . . . . . . . . 31

2.3 Fiber reinforced lamina - constitutive equations . . . . . . . . . . 36

2.4 Composite homogenization for multiple inclusion problems . . . . . . . . . 40

3 BEM for 3D anisotropic elasticity

3.1 Introduction . . . . . . . . . . . . . . . . . 43

3.2 The 3D anisotropic fundamental solutions . . . . . . . . . . . . 43

3.3 Fourier series representation $\ldots \ldots \ldots \ldots$. . . . . . . . . . . 47

3.4 Derivatives of the anisotropic fundamental solution . . . . . . . . . . . . 49

3.5 Discretization of the BIE . . . . . . . . . . . . . . 53

3.6 Multi-domain analysis for fiber-matrix problems . . . . . . . . . . 56

3.7 Numerical examples . . . . . . . . . . . . . . . . . . . . . 60

3.7.1 Example A: Validation of the anisotropic fundamental solution and its derivatives ..................... 60

3.7.2 Example B: BEM implementation - Isotropic material . . . . . . . . . . 64

3.7.3 Example C: BEM implementation - Alpha quartz crystal . . . . . . . . 66

3.7.4 Example D: BEM implementation - Generally anisotropic material . . . 68

3.7.5 Example E: Multi-domain approach . . . . . . . . . . . . 70 
4 The Boundary Element Method for 3D elastodynamics

4.1 Introduction . . . . . . . . . . . . . . . . . . . . . . . . 74

4.2 Integral representation . . . . . . . . . . . . . . . . . . 74

4.3 Cell integration method . . . . . . . . . . . . . . . . . 75

4.4 Dual Reciprocity Formulation $(\mathrm{DRM}) \ldots \ldots$. . . . . . . . . . 77

4.5 The Radial Integration Method (RIM) ～. . . . . . . . . . . . . . . 78

4.6 Dynamic analysis: transient analysis . . . . . . . . . . . . . . . . 82

4.6.1 Direct analysis and the Houbolt's algorithm . . . . . . . . . . . 82

4.7 Numerical examples . . . . . . . . . . . . . . . . . . . . . . . . . . . . . 84

4.7.1 Example A: Simple body force - gravity . . . . . . . . . . . . . . 84

4.7.2 Example B: Body forces on multi-domain anisotropic materials . . . 87

4.7.3 Example C: Transient dynamic analysis - Heaviside step load . . . . . . 91

4.7.4 Example D: Transient dynamic analysis - ramp load . . . . . . . . . . . 94

5 Fast BEM approach $\quad 97$

5.1 Introduction . . . . . . . . . . . . . . . . . . . . . . 97

5.2 Fast BEM approach . . . . . . . . . . . . . . . . . . . . . . . 97

5.2.1 Hierarchical clustering . . . . . . . . . . . . . . . 98

5.2.2 Adaptive Cross Approximation - ACA . . . . . . . . . . . . . . 99

5.3 ACA applied to a multiple inclusion 2D potential theory problem . . . . . . . . 103

5.4 Numerical examples . . . . . . . . . . . . . . . . . . . . . . . . . 104

5.4.1 Example A: Hierarchical clustering in fast BEM . . . . . . . . . . . 105

5.4.2 Example B: ACA applied to a multiple inclusion problem in 2D potential theory . . . . . . . . . . . . . . . . . 108

5.4.3 Example C: ACA applied to an anisotropic elastostatics problem . . . . 114

5.4.4 Example D: ACA applied to another generally anisotropic problem in elastostatics . . . . . . . . . . . . . . . . . 116

5.4.5 Example E: Fiber-matrix model . . . . . . . . . . . . . 118

6 Multi-scale failure analysis of laminated composites $\quad 124$

6.1 Introduction . . . . . . . . . . . . . . . . . . . . . . . . 124

6.2 Cohesive zone atomistic-molecular potential . . . . . . . . . . . . . . 124

6.3 Effective deformation gradient in the cohesive zone . . . . . . . . . . . . . 129

6.4 Cauchy-Born rule . . . . . . . . . . . . . . . . . . . . 132

6.5 Cohesive forces and failure criterion . . . . . . . . . . . . . . . . 134

6.6 Numerical examples . . . . . . . . . . . . . . . . . . . . . . 139

6.6.1 Laminate composite model . . . . . . . . . . . . . . . . . . 140

6.6.2 Implementation issues . . . . . . . . . . . . . . . . . . . 142 
6.6.3 Example A: Laminate composite . . . . . . . . . . . . . . . . . . . . 143

6.6.4 Example B: Fiber composite model . . . . . . . . . . . . . . . 149

6.6.5 Example C: Laminate Composite with a central flaw . . . . . . . . . . 154

7 Concluding Remarks 159

7.1 Final conclusions . . . . . . . . . . . . . . . . . . . . 159

7.2 Suggestions for further research f . . . . . . . . . . . . . . . 160

$\begin{array}{ll}\text { References } & 162\end{array}$ 


\section{Introduction}

The use of composite materials has increased considerably over recent years due to their relatively superior properties such as lightness, flexibility, durability, adaptability and mechanical resistance, among others. For example, the Brazilian composite industry billed US\$ 1.37 billion in 2012, a $4.6 \%$ growth when compared to the previous year, as stated by the Assosiação Latino-Americana de Materiais Compósitos (ALMANCO) ${ }^{1}$. Hence, accurately predicting its mechanical properties and failure prediction of a composite is of great importance. Most failure criteria are based on experimental data and generally applied to a specific type of material or load conditions. In many cases, the failure criteria give information of when the failure occurs, but do not give details of the specific failure location. A microscale approach appears as a good solution to locate the site of failure. Moreover, the main difficulty is to correctly couple the microscale with the whole structure being analysed. This type of analysis, for considering more than one scale is called multi-scale analysis. Multi-scale modeling is an area that is in rapid evolution. Despite the fact that multi-scale problems have been studied for a long time in mathematics, a recent tendency is the direct application of these principles in applied sciences, such as: material science, chemistry, fluid dynamics and biology, among others. According to Horstemeyer (2009), muti-scale modeling has several advantages, such as,

- Multi-scale modeling can reduce the product development time by alleviating costly trial and error iterations, hence, reducing the number of costly large systems scale experiments.

- Multi-scale modeling can reduce product costs through material innovations and process design.

- Multi-scale modeling can increase product quality and performance by providing more accurate predictions of response to design loads.

- Multi-scale modeling can help medical practice in making diagnostic and prognostic evaluations relate to the human body.

The development of new numerical techniques and increasing computational capacity has reached a stage where critical multi-scale problems can be addressed. However, computational cost is still a significant factor mainly because of the large number of degrees of freedom involved to obtain accurate solutions. This has motivated the development of acceleration techniques in order to decrease the computational cost and storage requirements with the same degree of accuracy.

\footnotetext{
${ }^{1}$ http: //www.almaco.org.br/
} 
Composites are usually made of polymeric materials and recently polymers have been analysed using multi-scale models. In the absence of strengthening or stiffening materials, its structure depends on the volume fractions of crystalline and/or amorphous constituents. Ismail et al. (2005) performed a hierarchical averaging scheme using wavelet transforms to study the multi-scale mechanical behavior of polymers, while, Theodurou (2005) used a hierarchical multi-scale approach to study the mechanical behavior of amorphous polymers. Yu and Fish (2002) developed a muti-scale, multiphysics concurrent method for analyzing viscoelasticity using asymptotic analysis to capture the spatially length scales. Molecular Dynamics (MD) were also used by Yanovsky (2005) to study micromechanical behavior of heterogeneous composite media taking into consideration atomic-molecular formations, as can be observed in Figure 1.1.

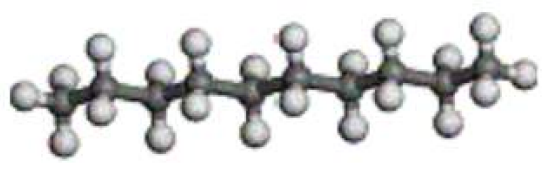

(a)

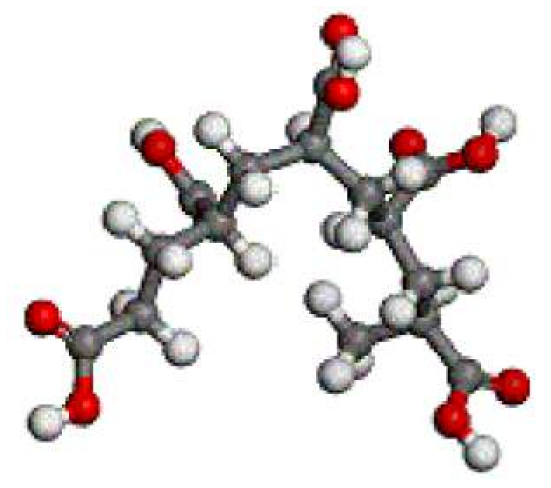

(c)

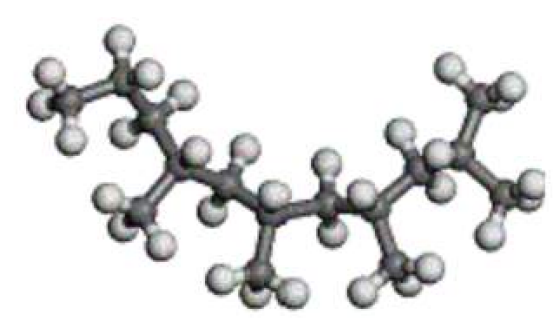

(b)

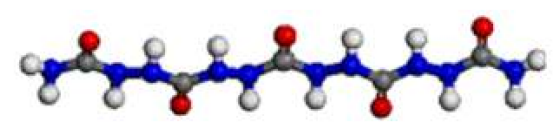

(d)

Figure 1.1: Fragment of polymer (oligomer) modeled by MD; (a) Polyethylene (PE); (b) Polypropylene (PP); (c) Polyacrylic acid (PAA); (d) Polurea (PU), (YANOVsKY, 2005).

MD was also used by Curgul et al. (2007) to perform simulations of amorphous polymer nanofibers to study the size-dependence properties. The fibers comprised chains with lengths ranging between 50 and 300 carbon atoms. They concluded that glass transition temperature of these amorphous nanofibers decreased with a decreasing fiber diameter. Composites made of polymers have been also extensively studied in recent years. Diverse multi-scale formulations have been developed, such as, top-down internal state variable approaches, self-consistent (homogenization) theories, and nanoscale quantum-molecular scale methods. The earliest works 
of multi-scale damage modeling of composites were probably those of Halpin et al. (1970), (HALPIN, 1972) and Hahn and Tsai (1974). In these models, polymer cracking, fiber breakage, interface debonding between the fiber and polymer matrix and delamination between ply layers were studied. Each of the different failure modes was represented by a length scale failure criterion formulated within a continuum, being, an early form of a hierarchical multi-scale method. Later, Halpin and Kardos (1976) described the relations of the Halpin-Tsai equations with selfconsistent methods and the micromechanics of Hill, (HILL, 1965). A numerical procedure for multiscale modeling to account for global and local interactions of composites was presented by Fish and Shek (2000). Later, Fish and Yu (2001) introduced a non-local damage model in a three-scale concurrent multi-scale methodology.

The homogenization theory have also been widely used in composite failure analysis. Belsky et al. (1995), for example, presented a homogenization method that included critical deformation modes, vibration and buckling modes; and delamination, debonding and microbuckling failure modes. Hassani (1998), Hassani and Hinton (1998a) and Hassani and Hinton (1998b) published a set of papers focusing on the analytical and numerical methods for the theory of homogenization and optimization. They showed that these methods can be used for topological features, such as geometries and microstructures, to bridge two different length scales. McCartney (2003) presented a compilation of several models for damage analysis in laminated composites. Homogenization techniques were used for determining ply properties for undamaged and initially damaged composite laminates. Thermal residual stresses were also considered. Matous et al. (2008) performed a multi-scale analysis on heterogeneous thin layers using homogenization theory for cohesive failure. Jin et al. (2008) used homogenization to model micro-stress distributions in unidirectional composites. They introduced the concept of amplification factor to correlate micro and macro stresses. Moreover, different unit cell models arrays were consider and validated with FEM. Some of the considered arrays can be observed in Figure 1.2.

Aboudi (1989), Aboudi (2004) and Paley and Aboudi (1992) developed various micromechanical FEM simulations at the mesoscale with the Generalized Method of Cells (GMC). Williams and Tippetts (2004) reviewed multi-scale approaches for polymer-based composites and proposed a three-scale method. The macro-scale was associated with the plate thickness and the meso-scale associated with the lamina thickness. A cohesive zone model was implemented with an adaptive meshing. The lowest length scale corresponded to a material point within each lamina, and was modeled by a stochastic homogenization theory. Composite nanomaterials were also widely studied. For example, Quian et al. (2002) developed multi-scale, multi-physics numerical tools to address simulations of carbon nanotubes and their effects in composite prop- 
(a)

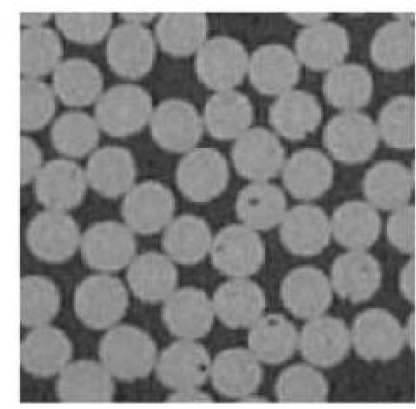

(c)

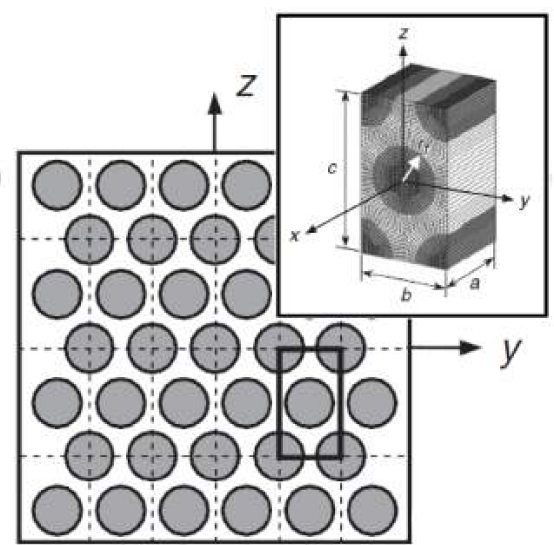

(b)
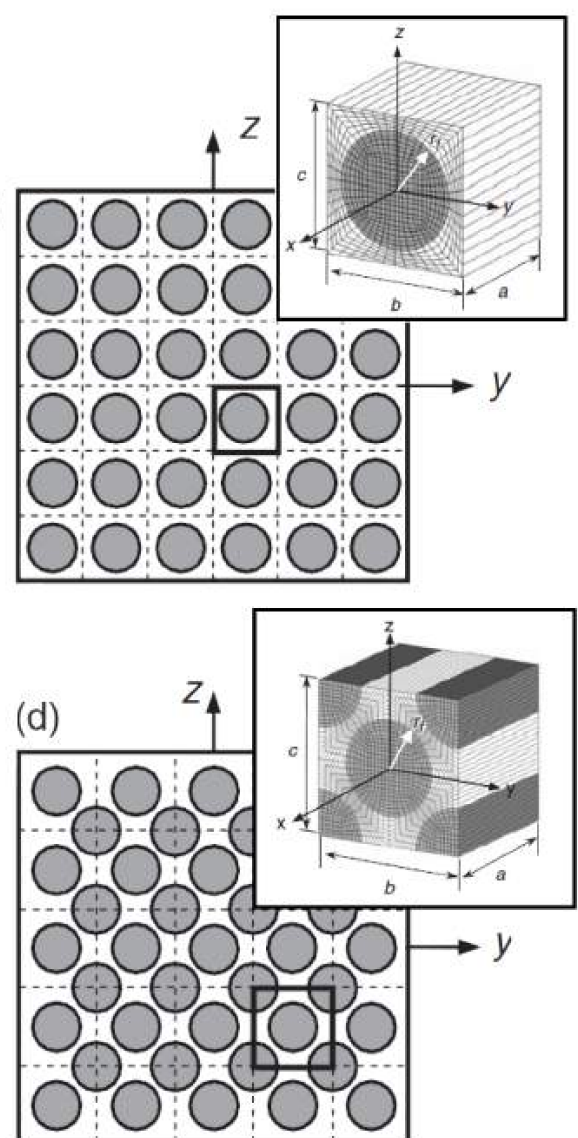

Figure 1.2: Comparison of real fiber arrangement and idealized fiber arrays; (a) Fiber array in real composite; (b) square fiber array; (c) hexagonal fiber array; (d) diamond fiber array, (JIN et al., 2008).

erties, such as Young's modulus, bending stiffness, buckling and strength, among others. Maiti (2008) also used multi-scale modeling of carbon nanotubes for microelectronics applications. Wescott (2006) used atomistic simulations, mesoscale simulations and FEM to examine the effects of nanotubes that were dispersed in polymers. A more detailed analysis of multi-scale modeling of diverse materials can be found in (HORSTEMEYER, 2009).

More recently, Gosh and Chaudhuri (2013) used the Cohesive Zone Model (CZM) with fracture mechanics to model failure in concrete composites. Kastner et al. (2013) modeled a representative volume element (RVE) with the extended finite element method (XFEM) to account for failure at the cohesive zone and applied their model to textile-reinforced composites. The fracture energy at the mixed-mode is also widely spread and applied, e.g., to graphite nanoplatelet polymer composites (SAFAEI et al., 2015), to cross-ply laminates (YANG et al., 2011) and to massive parallel simulations of composites (VIGUERAS et al., 2015), among others. However, few works take into account the real behaviour at the microscale. A 
multiscale cohesive zone model (MCZM) that considers the atomistic scale was proposed by (ZENG AND LI, 2010). More recently, this technique was coupled with BEM (GALVIS AND SOLLERO, 2014) for analysing failure by decohesion in 2D polycrystalline materials.

The Boundary Element Method (BEM) is in general very convenient for solving physical problems, especially those involving infinite or semi-infinite domains, such as those found in acoustic, soil-structure interaction, etc; and for stress concentration problems. This is mainly due to the use of the so-called fundamental solution, which is an analytical free-space solution of the governing differential equations for a source point excitation, hence, representing farfields and stresses very accurately, (KöGL AND GAUL, 2000a). The BEM is also attractive due to the reduction in the dimensionality of the numerical problem. In the case of two-dimensional problems, only the line-boundary of the total domain needs to be discretized into elements, while for three-dimensional problems, only the domain surface needs to be discretized. Therefore, when compared to other domain-based analysis techniques, a boundary analysis results in a substantial reduction in modelling effort, (ALIABADI, 2002). As an example, Liu (2009) modelled an engine-block to conduct a thermal analysis and compared results obtained with the Finite Element Method (FEM) and the Boundary Element Method (BEM). For the FEM analysis, he used the commercial software ANSYS with more than 363,000 volume elements and 1.5 million DOFs. For the BEM analysis, he used the Fast Multipole Method (FMM) with 42,000 constant surface elements (triangular constant elements) and the same number of DOFs, as can be seen in Figure 1.3. On a desktop PC, the FEM solution took 50 min to complete, whereas the BEM solution took only 16 min with a difference in the computed results of the temperature fields of less than $1 \%$. 


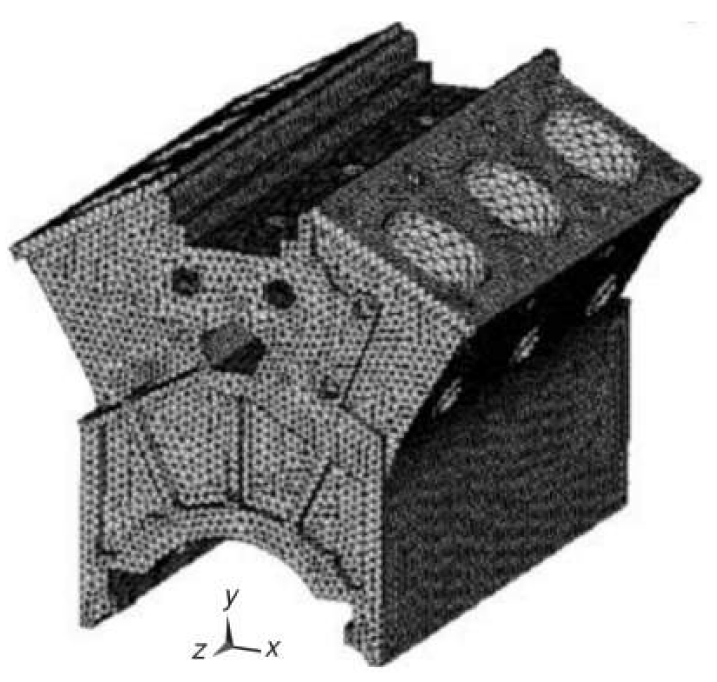

(a)

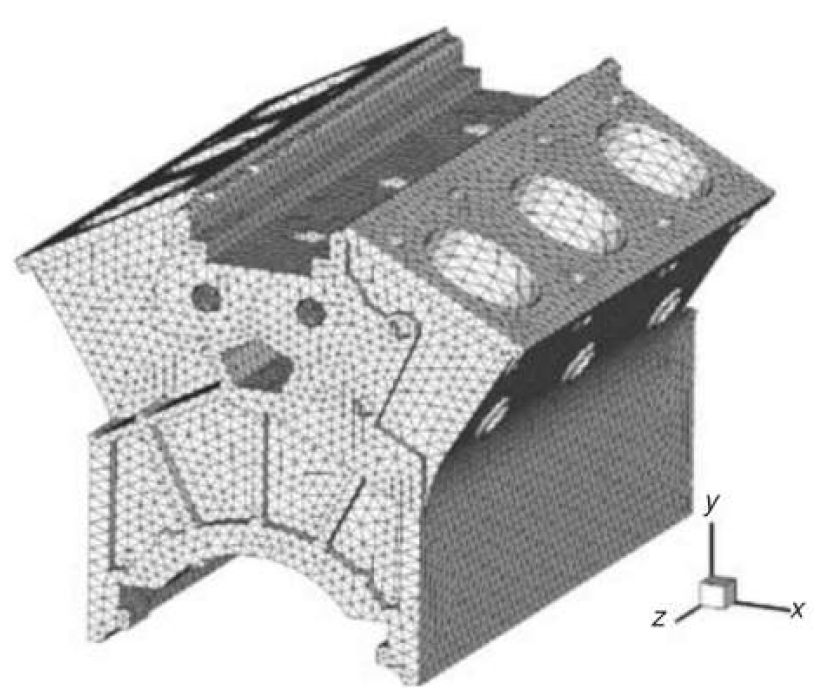

(b)

Figure 1.3: An engine block discretized using FEM and BEM; (a) FEM (363,000 volume elements/1.5 million DOFs); (b) BEM (42,000 surface elements/DOFs), (LIU, 2009).

The efficient computation of the fundamental solution, or Green's function, is crucial in the direct formulation of the BEM. For isotropic elasticity it can be represented in relatively simple explicit forms. In the case of general anisotropic solids, however, the Green's function is significantly more mathematically involved, particularly in 3D. The anisotropic fundamental solution in 3D was first presented by (LIFSHITZ AND ROZENZWEIG, 1947), but is not of closed form. This fundamental solution was employed by (WILSON AND CRUSE, 1978) in the first implementation for 3D general anisotropy in BEM. There were significant challenges in the development of efficient algorithms for the accurate numerical evaluation of this quantity and its derivatives. Attempts to improve on this matter have continued over the years, particularly to simplify analytically the Green's function into forms more amenable for numerical computations, see, e.g., (SALES AND GRAY, 1998), (TONON et al., 2001), (PHAN et al., 2004), (WANG AND DENDA, 2007). Explicit, closed form expressions of the Green's function and its derivatives were derived by (TING AND LEE, 1997) and (LEE, 2003) in terms of the Stroh's eigenvalues. However, the presence of high-order tensors and highly complex mathematical expressions for the derivatives, although straightforward to implement, may still be less than ideal for very efficient computations. These expressions were left unnoticed by the BEM community until (TAVARA et al., 2008) implemented it in BEM for the special case of transverse isotropy, and (SHIAH et al., 2008) and (TAN et al., 2009) did so for general anisotropy. Recognizing the periodic nature of the Green's function of (TING AND LEE, 1997) when expressed in spherical coordinates, the latter authors have very recently reformulated this fundamental solution and its derivatives by representing them by double Fourier series (Tan et al., 2013). This development 
has been shown to significantly reduce the computational effort for the accurate evaluation of these quantities. Recently, Shiah and Tan (2014) applied the Fourier series representation for solving the BIE for 3D thermoelasticity.

The Boundary Integral Equation (BIE) for elastodynamics may contain domain integrals. These integrals cannot be directly evaluated without destroying the notion of the BEM as a boundary solution technique and require a further transformation into boundary integrals. Some methods for this integral transformation are available in the literature, such as, the Fourier expansions, the Galerkin Vector Technique, the Multiple Reciprocity Method, the Cell integration method, the Dual Reciprocity Method (DRM) and the Radial Integration Method (RIM), among others. The latter two methods will be presented in the following chapters. The DRM is basically a generalized way of constructing some particular solutions that are used to solve non-linear and time-dependent problems, (Partridge et al., 1992). The DRM was introduced by Nardini and Brebbia (1982) for solving elastodynamic problems. It was further extended to time-dependent difussion problems by Wrobel and Brebbia (1987). A complete bibliography of the DRM applications is available in Partridge et al. (1992). Kögl and Gaul (2000a) introduced the DRM for dynamic analysis of anisotropic elastic solids. Albuquerque et al. (2002) further applied it to time dependent problems in anisotropic materials. Matsumoto et al. (2005) used the DRM for analysis of thermoelastic deformations in materials with properties that depend on temperature. The DRM was coupled with the multi-domain approach by Natalini and Popov (2007); they named the approach as DRM-MD and applied it to 3D advection-diffusion problems. Medeiros and Partridge (2007) applied the DRM to the method of fundamental solutions (MFS) and solve linear elastic 3D problems. The hybrid boundary node method (HBNM) was also coupled with the DRM by Yan et al. (2009). They applied the method to free vibration analysis. More recently, the DRM was applied to analyse free vibration of fluid-coupled Kirchhoff plates by Ugurlu (2015).

The RIM was initially proposed by Gao (2001). This approach is based on pure mathematical treatments, so it could be applied to transform any domain integral without the necessity of using the Laplace operator or particular solutions, (Gao, 2002). The RIM was applied to: dynamic problems of anisotropic plates by Albuquerque et al. (2007a), transient heat conduction problems by Yang and Gao (2010), numerical arbitrary singular domain integrals evaluation by Gao and Peng (2011) and acoustic eigenvalue problems by Qu et al. (2013). The last author used the RIM in order to eliminate the frequency dependency of the coefficient matrices in the traditional BEM. More recently, the RIM was applied to solving on-phase solidification and freezing problems by Yu et al. (2014); to dynamic coupled thermoelastic analysis under thermal shock loading by Gao et al. (2015) and for solving non-Fourier heat conduction problems 
by Yao et al. (2015).

There are few works that analyses elastodynamics problems in anisotropic materials using the BEM. Wang and Achenbach (1996) analysed scattering of elastic waves due to a crack in an infinite bi-dimensional anisotropic media. Saez and Dominguez (1999) presented a formulation of the scattering of harmonic waves due to a crack in three-dimensional transversely isotropic media using a numerical fundamental solution proposed by Wang and Achenbach (1995). This fundamental solution accounted for anisotropic materials, however it was used for transversely isotropic materials. Zhang (2000) presented an elastodynamic transient analysis for cracks in an infinite medium over the incidence of antiplane waves. Albuquerque and Sollero (1998) showed a transient formulation for two-dimensional elastodynamic problems. Then, this formulation was extended to account for fracture problems, (Albuquerque et al., 1999b),(Albuquerque et al., 1999a). Albuquerque et al. (2001) proposed a BEM formulation with DRM for elastodynamic anisotropic materials in the Laplace domain.

Over the years, the boundary element method (BEM) has been demonstrated to be a powerful numerical tool for the analysis and solution of many physical and engineering problems. However, a big disadvantage of BEM when compared with other numerical methods is the resultant fully populated and unsymmetric overall system matrix. For very large systems, this has implications on the memory requirements and solution times. Many research studies have been carried out to speed up the solution process. One popular approach is the Fast Multipole Method (FMM) (RoKHLIN, 1985); however, the main disadvantage of this method is the need of the harmonic expansion of the kernels. From the algebraic point of view, the integration of a degenerate kernel over a cluster of suitably selected boundary elements corresponds to the approximation of the related matrix block by a low rank block (BEBENDORF, 2000). As a consequence, it is possible to use purely algebraic algorithms to generate the approximation of suitable blocks of the collocation matrix, using only few entries of the original blocks (BEBENDORF AND RJASANOw, 2003). This technique is referred to as the Adaptive Cross Approximation (ACA). The ACA uses matrix hierarchization to reduce the storage requirement and the computational effort in the BEM analysis. The original ACA algorithm was proposed by Bebendorf (2000), and in Bebendorf and Rjasanow (2003). Several ACA algorithms and variations, as the so-called ACA+, are available in the literature (GRASEDYCK, 2005), (BEBENDORF AND GRZHIBOVSKIS, 2006), (KURZ et al., 2007). The algorithm used in this work for the low rank approximations is the same as that presented by Kurz et al. (2007). Moreover, results obtained after the low rank approximation of the admissible blocks by ACA, can be further recompressed, taking advantage of the reduced singular value decomposition (SVD) (Grasedyck, 2005), thereby decreasing the storage requirements. This also serves as a good pre-conditioner 
for iterative numerical solvers. Some works related to this topic may be found in (BENEDETTI et al., 2009), (HACKBUSCH et al., 2004). The ACA was applied to several BEM problems. It was applied to 3D anisotropic crack problems by Benedetti et al. (2009), to acoustic problems by Brancati et al. (2009), to three-dimensional elastodynamic crack problems by Benedetti and Aliabadi (2010). Milazzo et al. (2012) presented a fast BEM approach based on ACA to deal with anisotropic time-harmonic 3D elastodynamics. The same approach was further applied by Mallardo et al. (2012) to simulate noise control in an aircraft cabin. Rodríguez et al. (2013) applied the ACA to multiple inclusion potential problems, a fast BEM approach was also implemented. This scheme was successfully applied to the analysis of anisotropic solids using fundamental solutions based on double Fourier series. The ACA was also successfully applied to high order BEM for Maxwell problems by Rjasanow and Weggler (2013) and to the meshless method MFS for potential problems. More recently, Fu and Popov (2015) modified the ACA to account for the dynamic modelling of multiple bubbles.

\subsection{Motivation}

The present work has as its main motivations, the following topics:

- The BEM anisotropic implementation for elastostatics has been a subject of great interest for decades. The efficient and accurate computation of the fundamental solution and its derivatives is a big concern for the case of 3D general anisotropic solids because of their mathematical complexity.

- The non-symmetric and fully populated nature of BEM system equation matrices involve relatively high computer memory requirements and solution times for very large numerical problems such as those found in a multi-scale problem.

- The Boundary Integral Equation (BIE) for elastodynamics contains domain integrals. These integrals require to be transformed to the boundary in order to apply the BEM.

- Most material failure criteria are based on experimental data and generally applied to a specific type of material or load conditions. In many cases, there is information of when the failure occurs but not of the failure location. 


\subsection{Objectives}

The present work has as its main objective the development of computational tools capable of facilitating multi-scale failure analysis in composites through numerical modelling using the Boundary Element Method (BEM). The meso-scale will be modelled using the fundamental solution based on double Fourier series and a fast BEM approach based on hierarchical matrices and the Adaptive Cross Approximation (ACA). Moreover, dynamic effects will be considered by means of the Dual Reciprocity Method (DRM) and the Radial Integration Method (RIM). Finally, realistic combinations of fiber-matrix models will be tested. More specifically, these objectives include:

- Implementation of the 3D generally anisotropic fundamental solution based on double Fourier series.

- Implementation of a fast BEM approach based on hierarchical matrices and the Adaptive Cross Approximation (ACA), as well as to adapt the method to multi-domain problems.

- Implementation of dynamic inertial effects in the equation of motion by means of the DRM and the RIM methods.

- Analyse failure in laminate composites with real material properties using a multi-scale approach.

- Develop a robust BEM programming code for multi-scale analysis using Fortran 90, the GNU compiler gfortran and multiprocessor routines based on the OpenMP interface.

○ Publication in international journals and conference proceedings.

\subsection{Contributions}

Some of the main novel contributions of the present Ph.D. thesis are:

- The combination of a Fast BEM approach with the double Fourier representation of the displacement fundamental solution and its derivatives.

- The ACA within the Fast BEM approach adaptation to 2D multiple inclusion potential problems, 3D single-domain, 3D multi-domain, short fibers-matrix models and composite laminates. 
- Dynamic effects are treated in 3D anisotropic elasticity. The transformation of the domain integrals in the boundary integral equation into boundary ones was by the DRM and the RIM.

- To the best of author's knowledge the present thesis is the first work to deal with multiscale failure in the dynamic analysis of general anisotropy with a fast BEM approach.

The above-mentioned contributions resulted in some publications in conferences and international journals. The thesis has hitherto generated the following complete paper publications in conferences:

1. Galvis, A.F., Rodríguez, R.Q. and Sollero, P. Intergranular fracture in polycrystalline materials using the dual reciprocity boundary element method. Accepted for publication in the 16th International Conference on Boundary Element and Meshless Techniques BETEQ 2015, Valencia, Spain, 2015.

2. Rodríguez, R.Q., Tan, C.L., Sollero, P. and Albuquerque, E.L. Acceleration scheme for multi-domain 3D generally anisotropic solids using double Fourier series and adaptive cross approximation. Proceedings of the XXXV Iberian Latin American Congress on Computational Methods in Engineering - CILAMCE 2014, Fortaleza-Ceara, Brazil, 2326 Nov. 2014.

3. Rodríguez, R.Q., Tan, C.L., Sollero, P. and Albuquerque, E.L. Analysis of 3D anisotropic solids using fundamental solutions based on Fourier series and the adaptive cross approximation method, Proceedings of the 15th International Conference on Boundary Element and Meshless Techniques - BETEQ 2014, pp. 87-92, Florence, 15-17 July 2014.

4. Galvis, A.F., Rodríguez, R.Q., Sollero, P. and Albuquerque, E.L. Multidomain Formulation of BEM Analysis Applied to Large-Scale Polycrystalline Materials, Proceedings of the 14th International Conference on Boundary Element and Meshless Techniques BETEQ 2013, pp.32-37, Paris, France, 2013.

5. Rodríguez, R.Q., Galvis, A.F., Sollero, P. and Albuquerque, E.L. Analysis of Multiple inclusion potential problems by the Adaptive Cross Approximation(ACA), Proceedings of the 14th International Conference on Boundary Element and Meshless Techniques BETEQ 2013, pp.225-230, Paris, France, 2013.

6. Moraes, D.H., Rodríguez, R.Q., Sollero, P. and Albuquerque, E.L., Multiscale Analysis of Failure in Composite Materials, 1 st Brazilian Conference on Composite Materials BCCM1, Natal, Brasil, 2012. 
7. Rodríguez, R.Q., Sollero, P. and Albuquerque, E.L., Analysis of Anisotropic Symmetric Plates by the Adaptive Cross Approximation, Proceedings in Advances in Boundary Element \& Meshless Techniques - BETEQ 2012, pp. 303-310, 2012.

8. Gama, A.B., Ozelo, R.R.M., Rodríguez, R.Q. and Sollero P., Multiscale analyses applied to rubber compounds: A literature survey, III Simposio en Mecánica de Materiales y Estructuras Continuas - SMEC 2011, Colombia, 2011.

The thesis work has also generated the following extended abstract publication at a conference:

1. Rodríguez, R.Q., Sollero, P., Tan, C.L. and Albuquerque, E.L. ACA applied to 3D anisotropic stress analysis of fiber-matrix composites using the multi-domain BEM, International Conference on Computational \& Experimental Engineering and Sciences ICCES 2015, Reno NV, USA, 20-24 July 2015.

The thesis work also generated the following publications in journals:

1. Rodríguez, R.Q., Tan, C.L., Sollero, P. and Albuquerque, E.L. Analysis of 3D Anisotropic Solids Using Fundamental Solutions Based on Fourier Series and the Adaptive Cross Approximation Method . Computer Modeling in Engineering \& Sciences, 102, 359$372,2014$.

2. Rodríguez, R.Q., Galvis, A.F., Sollero, P. and Albuquerque, E.L. Analysis of Multiple Inclusion Potential Problems by the Adaptive Cross Approximation Method. Computer Modeling in Engineering \& Sciences, 96, pp. 259-274, 2013.

3. Galvis, A.F., Rodríguez, R.Q., Sollero, P. and Albuquerque, E.L. Multidomain Formulation of BEM Analysis Applied to Large-Scale Polycrystalline Materials. Computer Modeling in Engineering \& Sciences, 96, pp. 103-115, 2013.

\subsection{Thesis organization}

The present thesis is composed of seven chapters. In the first chapter, a brief state of the art is presented. Then, the motivation, objectives and contributions of the work are described. In the 
second chapter, the governing equations for elastic anisotropic materials are briefly reviewed. The third chapter presents the BEM for 3D elastostatics; in this chapter the generally anisotropic fundamental solution based on double Fourier series is further elaborated. The multi-domain analysis is also explored and adapted to account for fiber-matrix problems. Numerical results are shown in order to validate the mentioned formulation. In the fourth chapter, the BEM for 3D elastodynamics is briefly reviewed. Two methods, namely the Dual Reciprocity Method (DRM) and the Radial Integration Method (RIM), are presented for transforming domain integrals into boundary integrals for the inertial dynamic components in the equation of motion. Numerical examples are also presented in this chapter. In the next chapter, the fast BEM approach is studied. The acceleration process is accounted by means of hierarchical clustering and low rank approximations using the Adaptive Cross Approximation (ACA). Numerical examples are shown and a brief illustration of how the method works is also presented. In the sixth chapter, the multi-scale failure criterion is exposed. The cohesive law based on an adapted intermolecular potential is adopted and numerical examples with realistic combinations of fiber-matrix materials for composite laminates are shown. Finally, the conclusions of the work, as well as, suggestions for further research are presented in the last chapter. 


\section{Constitutive equations and equilibrium for anisotropic materials}

\subsection{Introduction}

The analytical and numerical formulations implemented in this thesis will be presented and discussed in the chapters that follow. It is useful, however, to, first, review a few of the key governing equations in anisotropic elasticity and in the mechanics of composite laminates. The former will be expanded further when the implementation of the BEM for 3D general anisotropic elasticity is presented in the next chapter. Also reviewed here, in Section 2.5, is the homogenization of the thermal conductivity of a composite with multiple inclusions in $2 \mathrm{D}$. Although a deviation from elasticity, it provides the relevant background for a validation example treated in Chapter 3 when dealing with the ACA scheme with BEM.

\subsection{Anisotropic elasticity}

The classical Cauchy's equation of motion may be as,

$$
\sigma_{i j, j}+\rho b_{i}-\rho \ddot{u}_{i}=0,
$$

where $\sigma_{i j}$ is the Cauchy stress tensor, $b_{i}$ are the body forces, $\rho$ is the mass density and $u_{i}$ is the displacement vector. The two dots denote double differentiation with respect to time. This expression comes from the conservation of linear momentum. The conservation of angular momentum also establishes that

$$
\sigma_{i j}=\sigma_{j i}
$$

In linear elasticity, the displacement vector and its derivatives are considered as infinitesimals. The infinitesimal strain tensor,

$$
\varepsilon_{i j}=\frac{1}{2}\left(u_{i, j}+u_{j, i}\right),
$$


describes the kinematics of deformation. For given displacements, $u_{i}$, the strain components, $\varepsilon_{i j}$, can be determined from Equation (2.3). However, for prescribed strain components this expression represents a system of partial differential equations that is not expected to have an unique solution. Therefore, in order to ensure uniqueness of displacements, some restrictions must be imposed on the strain components $\varepsilon_{i j}$. These restrictions are known as the compatibility conditions,

$$
\varepsilon_{i j, k l}+\varepsilon_{k l, i j}-\varepsilon_{i k, j l}-\varepsilon_{j l, i k}=0
$$

The relation between stress and displacement is given by the generalized Hooke's law for an anisotropic body as follows,

$$
\sigma_{i j}=C_{i j k l} \varepsilon_{k l}
$$

where $C_{i j k l}$ is the material stiffness tensor and it has the symmetry $C_{i j k l}=C_{j i k l}=C_{k l i j}$. This reduces the number of independent elastic constants from 81 to 21. Equation (2.5) can be rewritten in matrix form as,

$$
\left\{\begin{array}{l}
\sigma_{11} \\
\sigma_{22} \\
\sigma_{33} \\
\sigma_{23} \\
\sigma_{13} \\
\sigma_{12}
\end{array}\right\}=\left\{\begin{array}{llllll}
C_{1111} & C_{1122} & C_{1133} & C_{1123} & C_{1113} & C_{1112} \\
C_{1122} & C_{2222} & C_{2233} & C_{2223} & C_{2213} & C_{2212} \\
C_{1133} & C_{2233} & C_{3333} & C_{3323} & C_{3313} & C_{3312} \\
C_{1123} & C_{2223} & C_{3323} & C_{2323} & C_{2313} & C_{2312} \\
C_{1113} & C_{2213} & C_{3313} & C_{2313} & C_{1313} & C_{1312} \\
C_{1112} & C_{2212} & C_{3312} & C_{2312} & C_{1312} & C_{1212}
\end{array}\right\}\left\{\begin{array}{c}
\varepsilon_{11} \\
\varepsilon_{22} \\
\varepsilon_{33} \\
2 \varepsilon_{23} \\
2 \varepsilon_{13} \\
2 \varepsilon_{12}
\end{array}\right\}
$$

Equation (2.5) can also be written as,

$$
\varepsilon_{i j}=S_{i j k l} \sigma_{k l}
$$

where $S_{i j k l}$ is known as the elastic flexibility or compliance tensor. As for the stiffness tensor, it has 21 independent elements. This tensor can also be written in matrix form, 


$$
\left\{\begin{array}{c}
\varepsilon_{11} \\
\varepsilon_{22} \\
\varepsilon_{33} \\
2 \varepsilon_{23} \\
2 \varepsilon_{13} \\
2 \varepsilon_{12}
\end{array}\right\}=\left\{\begin{array}{cccccc}
S_{1111} & S_{1122} & S_{1133} & S_{1123} & S_{1113} & S_{1112} \\
S_{1122} & S_{2222} & S_{2233} & S_{2223} & S_{2213} & S_{2212} \\
S_{1133} & S_{2233} & S_{3333} & S_{3323} & S_{3313} & S_{3312} \\
2 S_{1123} & 2 S_{2223} & 2 S_{3323} & 4 S_{2323} & 4 S_{2313} & 4 S_{2312} \\
2 S_{1113} & 2 S_{2213} & 2 S_{3313} & 4 S_{2313} & 4 S_{1313} & 4 S_{1312} \\
2 S_{1112} & 2 S_{2212} & 2 S_{3312} & 4 S_{2312} & 4 S_{1312} & 4 S_{1212}
\end{array}\right\}\left\{\begin{array}{l}
\sigma_{11} \\
\sigma_{22} \\
\sigma_{33} \\
\sigma_{23} \\
\sigma_{13} \\
\sigma_{12}
\end{array}\right\}
$$

Using the reduced tensorial notation (Voigt notation), see e.g. Lekhnitskii (1981), Equation (2.8) can be rewritten as,

$$
\left\{\begin{array}{l}
\varepsilon_{1} \\
\varepsilon_{2} \\
\varepsilon_{3} \\
\varepsilon_{4} \\
\varepsilon_{5} \\
\varepsilon_{6}
\end{array}\right\}=\left\{\begin{array}{llllll}
a_{11} & a_{12} & a_{13} & a_{14} & a_{15} & a_{16} \\
a_{12} & a_{22} & a_{23} & a_{24} & a_{25} & a_{26} \\
a_{13} & a_{23} & a_{33} & a_{34} & a_{35} & a_{36} \\
a_{14} & a_{24} & a_{34} & a_{44} & a_{45} & a_{46} \\
a_{15} & a_{25} & a_{35} & a_{45} & a_{55} & a_{56} \\
a_{16} & a_{26} & a_{36} & a_{46} & a_{56} & a_{66}
\end{array}\right\}\left\{\begin{array}{c}
\sigma_{1} \\
\sigma_{2} \\
\sigma_{3} \\
\sigma_{4} \\
\sigma_{5} \\
\sigma_{6}
\end{array}\right\}
$$

where,

$$
\left\{\begin{array}{l}
\varepsilon_{1} \\
\varepsilon_{2} \\
\varepsilon_{3} \\
\varepsilon_{4} \\
\varepsilon_{5} \\
\varepsilon_{6}
\end{array}\right\}=\left\{\begin{array}{c}
\varepsilon_{11} \\
\varepsilon_{22} \\
\varepsilon_{33} \\
2 \varepsilon_{23} \\
2 \varepsilon_{13} \\
2 \varepsilon_{12}
\end{array}\right\}
$$

and, 


$$
\left\{\begin{array}{l}
\sigma_{1} \\
\sigma_{2} \\
\sigma_{3} \\
\sigma_{4} \\
\sigma_{5} \\
\sigma_{6}
\end{array}\right\}=\left\{\begin{array}{l}
\sigma_{11} \\
\sigma_{22} \\
\sigma_{33} \\
\sigma_{23} \\
\sigma_{13} \\
\sigma_{12}
\end{array}\right\}
$$

The elastic coefficients of the compliance tensor can be further expressed in terms of engineering constants,

$$
\begin{array}{ll}
a_{11}=1 / E_{1} & a_{12}=\nu_{12} / E_{1}=-\nu_{21} / E_{2} \\
a_{13}=-\nu_{31} / E_{1}=-\nu_{13} / E_{3} & a_{14}=\eta_{23,1} / E_{1}=\eta_{1,23} / G_{23} \\
a_{15}=\eta_{32,1} / E_{1}=\eta_{1,32} / G_{23} & a_{16}=\eta_{12,1} / E_{1} \\
a_{22}=1 / E_{2} & a_{23}=\nu_{32} / E_{2}=-\nu_{23} / E_{3} \\
a_{24}=\eta_{23,1} / E_{2}=\nu_{23,3} / G_{23} & a_{25}=\eta_{31,2} / E_{2}=\eta_{2,31} / G_{13} \\
a_{26}=\eta_{12,2} / E_{2}=\eta_{2,12} / G_{12} & a_{33}=1 / E_{3} \\
a_{34}=\eta_{23,3} / E_{3}=\eta_{3,23} / G_{12} & a_{35}=\eta_{31,1} / E_{3}=\eta_{3,31} / G_{13} \\
a_{36}=\eta_{12,3} / E_{3}=\eta_{3,12} / G_{12} & a_{44}=1 / G_{23} \\
a_{45}=\zeta_{32,23} / G_{23}=\zeta_{23,31} / G_{13} & a_{46}=\zeta_{12,23} / G_{23}=\zeta_{23,12} / G_{12} \\
a_{55}=1 / G_{13} & a_{56}=\zeta_{12,31} / G_{13}=\zeta_{31,12} / G_{12} \\
a_{66}=1 / G_{12} &
\end{array}
$$

where $E_{k}$ are the longitudinal elasticity modulus, or Young's modulus, along the respective coordinate axis $x_{k}, G_{i j}$ is the shear modulus, or Coulomb modulus, for planes defined by $x_{i} x_{j}$ coordinate axes. $\nu_{i j}$ are the Poisson's ratios, which characterize the contraction in the direction of one axis when tension is applied in the direction of another axis. These constants are wellknown for an isotropic body. The constants $\eta_{i j, k}$ are known as the mutual influence coefficients of the first kind; they denote the extension in the direction of $x_{k}$ coordinate produced by shearing stresses acting in the coordinate plane $x_{i} x_{j}$. The constants $\eta_{i, j k}$ are known as the mutual influence coefficients of the second kind and denote the shear in the coordinate plane $x_{j} x_{k}$ due to a normal stress acting on the $x_{i}$ coordinate direction. Finally, $\zeta_{i j, k l}$ are called Chentsov's coefficients and they represent the shear deformation in planes parallel to the coordinates planes produced by the shearing stresses acting in the other planes parallel to the coordinate planes.

It is worth to point out that a further reduction of the number of independent elastic con- 
stants is possible if there are symmetries in the material. If there exists symmetry with respect to three mutually perpendicular axes, the material is called orthotropic and the number of independent constant is reduced to nine. If the material exhibits a rotational elastic symmetry about one of the coordinate axe, the material is named transversely isotropic and the number of independent constants is reduced to five. Finally, if the symmetry exists in all directions, the material is called isotropic and only two independent constants are required to describe the material behavior.

Substituting Equations (2.3) and (2.5) into Equation (2.1), we obtain the linear field equations of elastodynamics,

$$
C_{i j k l} u_{k, j l}+p_{i}=0
$$

where $p_{i}=\rho\left(b_{i}-\ddot{u}_{i}\right)$ is the term with all body forces. Equation (2.13) describes the motion of a linear-elastic body in terms of the displacement and are known as Navier's equations (GAUL et al., 2003).

If a unit concentrated force $\mathbf{f}=\mathbf{1}$ is applied at the origin $\mathbf{x}=\mathbf{0}$, when $p_{i}=0$ (i.e. zero body force and in statics condition), Equation (2.13) can be written as, (TING AND LEE, 1997),

$$
C_{i j k l} u_{k, l j}=-\delta\left(x_{1}\right) \delta\left(x_{2}\right) \delta\left(x_{3}\right) f_{i}
$$

where $\delta(x)$ is the Dirac delta function. The solution to Equation (2.14) is the fundamental solution and is the simplest conceivable non-trivial solution to the homogeneous equation in Equation (2.13)

Taking the Fourier transform of Equation (2.14) with respect to $x_{1}, x_{2}$ and $x_{3}$, solving for the transformed $u_{k}$ and performing the inverse transform leads to

$$
\mathbf{u}=\frac{1}{(2 \pi)^{3}} \iiint \mathbf{Z}^{-1}\left(\mathbf{n}^{*}\right) \mathbf{f} e^{-i \mathbf{n}^{*} \cdot \mathbf{x}} \mathrm{d} n_{1}^{*} \mathrm{~d} n_{2}^{*} \mathrm{~d} n_{3}^{*}
$$

where 


$$
Z_{i k}\left(\mathbf{n}^{*}\right)=C_{i j k l} n_{j}^{*} n_{l}^{*}
$$

Equation (2.16) is reducible to, (LIFSHITZ AND RoZENZWEIG, 1947),

$$
\mathbf{u}=\frac{1}{8 \pi^{2} r} \int_{s} \mathbf{Z}^{-1}\left(\mathbf{n}^{*}\right) \mathbf{f} \mathrm{d} s
$$

where $r=|\mathbf{x}|$ and the integral is taken around the unit circle $\left|\mathbf{n}^{*}\right|=1$ on the plane normal to $\mathbf{x}$. Equation (2.17) can be also written as,

$$
\begin{gathered}
\mathbf{u}=\mathbf{G}(\mathbf{x}) \mathbf{f}, \\
\mathbf{G}(\mathbf{x})=\frac{1}{8 \pi^{2} r} \int_{s} \mathbf{Z}^{-1}\left(\mathbf{n}^{*}\right) \mathrm{d} s,
\end{gathered}
$$

where $\mathbf{G}(\mathbf{x})$ is the Green's function for the infinite space. A further discussion and analysis of this expression will be given in the next chapter.

\subsection{Fiber reinforced lamina - constitutive equations}

A lamina consists of aligned stiff fibers embedded in a resin matrix and several laminae, with fibers in each array differently, are bonded together to form a laminate. Each lamina is usually considered as transversely isotropic or in some cases as orthotropic. Its principal axes are usually also at an angle with respect to the main reference axes of the laminate. The reader is referred to (ASHTON et al.; ZHEN AND BREBBIA, 1968; 1988) for more details. For illustration the orthotropic case will be considered for deducing the stress-strain relation for the lamina. The Hooke's law for a homogeneous orthotropic lamina which has its axes aligned with the reference axes , may be written as 


$$
\left\{\begin{array}{l}
\sigma_{1} \\
\sigma_{2} \\
\sigma_{3} \\
\sigma_{4} \\
\sigma_{5} \\
\sigma_{6}
\end{array}\right\}=\left\{\begin{array}{cccccc}
c_{11} & c_{12} & c_{13} & 0 & 0 & 0 \\
c_{12} & c_{22} & c_{23} & 0 & 0 & 0 \\
c_{13} & c_{23} & c_{33} & 0 & 0 & 0 \\
0 & 0 & 0 & c_{44} & 0 & 0 \\
0 & 0 & 0 & 0 & c_{55} & 0 \\
0 & 0 & 0 & 0 & 0 & c_{66}
\end{array}\right\}\left\{\begin{array}{l}
\varepsilon_{1} \\
\varepsilon_{2} \\
\varepsilon_{3} \\
\varepsilon_{4} \\
\varepsilon_{5} \\
\varepsilon_{6}
\end{array}\right\}
$$

where the components $c_{i j}$ are the components of the elasticity tensor or stiffness matrix, which is the inverse of the compliance matrix, or

$$
\left[Q_{i j}\right]=\left[a_{i j}\right]^{-1}
$$

The components of the stiffness matrix are given by, (Ashton et al., 1968),

$$
\begin{array}{lll}
c_{11}=\frac{1-\nu_{23} \nu_{32}}{E_{1} E_{3} \Delta} & c_{12}=\frac{\nu_{23}+\nu_{31} \nu_{23}}{E_{2} E_{3} \Delta} & c_{13}=\frac{\nu_{31}+\nu_{21} \nu_{32}}{E_{2} E_{3} \Delta} \\
c_{22}=\frac{1-\nu_{31} \nu_{13}}{E_{3} E_{1} \Delta} & c_{23}=\frac{\nu_{32}+\nu_{31} \nu_{12}}{E_{3} E_{1} \Delta} & c_{33}=\frac{1-\nu_{12} \nu_{21}}{E_{1} E_{2} \Delta} \\
c_{44}=2 G_{23} & c_{55}=2 G_{31} & c_{66}=2 G_{12}
\end{array} .
$$

Equation (2.20) has nine independent elastic constants, which are the Young's moduli $E_{1}$, $E_{2}$ and $E_{3}$, the shear moduli $G_{12}, G_{23}$ and $G_{13}$, and the Poisson's ratios $\nu_{12}, \nu_{23}$ and $\nu_{13}$. The values of $\nu_{21}, \nu_{32}$ and $\nu_{31}$ can be obtained from the following equivalence,

$$
\begin{aligned}
& \nu_{21} E_{1}=\nu_{12} E_{2} \\
& \nu_{23} E_{3}=\nu_{32} E_{2} \\
& o n \nu_{31} E_{1}=\nu_{13} E_{3}
\end{aligned} .
$$

A lamina whose principal and reference axes are aligned is called specially orthotropic. When the axes are not aligned, see Figure 2.1, the constitutive equation for each lamina must be transformed to the laminate reference axes. 


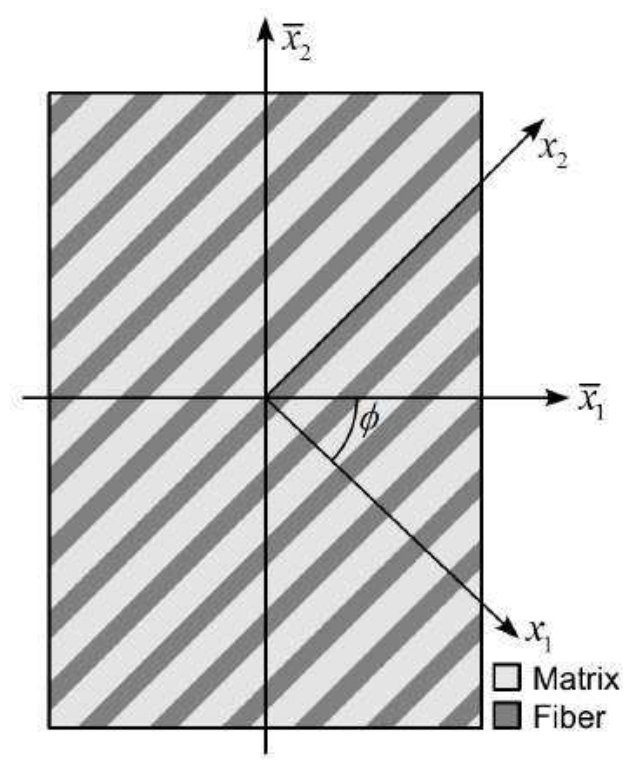

Figure 2.1: Lamina principal axes $\left(x_{1}\right.$ and $\left.x_{2}\right)$ and laminate reference axes $\left(\bar{x}_{1}\right.$ and $\left.\bar{x}_{2}\right)$.

The transformation can be expressed by

$$
\left[\sigma_{i j}\right]=\left[\bar{c}_{i j}\right] \varepsilon_{j}
$$

where $\left[\bar{c}_{i j}\right]$ is the contracted Voigt notation of $C_{i j k l}$. Thus,

$$
\left[\bar{c}_{i j}\right]_{l}=[T]_{l}\left[c_{i j}\right]_{l}[T]_{l}^{\mathrm{T}}
$$

is the stiffness matrix after the rotation of fibers. The index $l$ in Equations (2.24) and (2.25) refers to an individual lamina in the laminate. The transformation matrix $[T]_{l}$ is given by, (TING, 1996),

$$
[T]_{l}=\left[\begin{array}{cc}
{[T]_{1}} & 2[T]_{2} \\
{[T]_{3}} & {[T]_{4}}
\end{array}\right]
$$

where 


$$
\begin{gathered}
{[T]_{1}=\left[\begin{array}{lll}
\Omega_{11}^{2} & \Omega_{12}^{2} & \Omega_{13}^{2} \\
\Omega_{21}^{2} & \Omega_{22}^{2} & \Omega_{23}^{2} \\
\Omega_{31}^{2} & \Omega_{32}^{2} & \Omega_{33}^{2}
\end{array}\right],} \\
{[T]_{2}=\left[\begin{array}{lll}
\Omega_{12} \Omega_{13} & \Omega_{13} \Omega_{11} & \Omega_{11} \Omega_{12} \\
\Omega_{22} \Omega_{23} & \Omega_{23} \Omega_{21} & \Omega_{21} \Omega_{22} \\
\Omega_{32} \Omega_{33} & \Omega_{33} \Omega_{31} & \Omega_{31} \Omega_{32}
\end{array}\right],} \\
{[T]_{3}=\left[\begin{array}{lll}
\Omega_{21} \Omega_{31} & \Omega_{22} \Omega_{32} & \Omega_{23} \Omega_{33} \\
\Omega_{31} \Omega_{11} & \Omega_{32} \Omega_{12} & \Omega_{33} \Omega_{13} \\
\Omega_{11} \Omega_{21} & \Omega_{12} \Omega_{22} & \Omega_{13} \Omega_{23}
\end{array}\right],} \\
{[T]_{4}=\left[\begin{array}{lll}
\Omega_{22} \Omega_{23}+\Omega_{23} \Omega_{32} & \Omega_{23} \Omega_{31}+\Omega_{21} \Omega_{33} & \Omega_{21} \Omega_{32}+\Omega_{22} \Omega_{31} \\
\Omega_{32} \Omega_{13}+\Omega_{33} \Omega_{12} & \Omega_{33} \Omega_{11}+\Omega_{31} \Omega_{13} & \Omega_{31} \Omega_{12}+\Omega_{32} \Omega_{11} \\
\Omega_{12} \Omega_{23}+\Omega_{13} \Omega_{22} & \Omega_{13} \Omega_{21}+\Omega_{11} \Omega_{23} & \Omega_{11} \Omega_{22}+\Omega_{12} \Omega_{21}
\end{array}\right] .}
\end{gathered}
$$

where the matrix $\Omega_{i j}$ depends on the rotation reference axis,

$$
\begin{aligned}
& {\left[\Omega_{i j}\right]_{1}=\left[\begin{array}{ccc}
1 & 0 & 0 \\
0 & \cos \theta & \sin \theta \\
0 & -\sin \theta & \cos \theta
\end{array}\right],} \\
& {\left[\Omega_{i j}\right]_{2}=\left[\begin{array}{ccc}
\cos \theta & 0 & -\sin \theta \\
0 & 1 & 0 \\
\sin \theta & 0 & \cos \theta
\end{array}\right],} \\
& {\left[\Omega_{i j}\right]_{3}=\left[\begin{array}{ccc}
\cos \theta & \sin \theta & 0 \\
-\sin \theta & \cos \theta & 0 \\
0 & 0 & 1
\end{array}\right] .}
\end{aligned}
$$

In the above, $\left[\Omega_{i j}\right]_{1}$ corresponds to a rotation on the $x_{1}$ coordinate axis, $\left[\Omega_{i j}\right]_{2}$ corresponds to a rotation around the $x_{2}$ axis, and $\left[\Omega_{i j}\right]_{3}$ corresponds to a rotation around the $x_{3}$ axis. $\theta$ is the 
direction of rotation around the selected axis.

\subsection{Composite homogenization for multiple inclusion problems}

A brief review of the theory of composite homogenization is presented here. Some analytical formulas are shown with the intention of future validation of the BEM model when dealing with the acceleration scheme.

In a general multiple inclusion problem the center of each fiber can be randomly deviated within a circle of diameter $d$ whereas these circles themselves form a regular square lattice of the period $l$. This kind of a microstructure is usually referred as shaking-geometry composite (Berlyand And Mityushev, 2001), (Berlyand And Mityushev, 2005). As pointed out by (ANDRIANOV et al., 2008) and (KALAMKAROV et al., 2009), the random shaking of the fibers about the periodic lattice could be due to production or deliberate technical reasons. The deviation parameter $\delta=d / l$ describes the non-regularity rate of the structure and its maximum value $\delta_{\max }$ is determined by the case when fibres almost touch each other. Higher values of $\delta$ are not allowed since they would lead to inter-penetration of the fibers (percolation). A simple geometrical calculation give, (KALAMKAROV et al., 2009),

$$
\delta_{\max }=1-\sqrt{\frac{c}{c_{\max }}}
$$

where $c=\pi a^{2} / l^{2}$ is the volume fraction of the fibers, $c_{\max }=\pi / 4$ is the maximal volume fraction of the fibers in the case of a perfectly regular material $(\delta=0)$.

Kozlov (KoLZLOV, 1989) showed for the case of dilute components that a regular lattice possesses the extreme effective properties among the corresponding shaking-geometry random structures. Later, Berlyand and Mityushev (BERLyAnd AND Mityushev, 2001), (BERlyAnd AND Mityushev, 2005) generalized Kozlov's result for the case of non-dilute components. Therefore, a solution for the perfectly regular lattice can be considered as a lower bound on the effective transport coefficient, (KALAMKAROV et al., 2009), (ANDRIANOV et al., 2008). The upper bound can be obtained using the security-spheres approach. Originally created by Keller et al. (KELLER et al., 1967), in this approach, the upper bound can be obtained by replacing the input non-regular assembly of fibres of radius $a$ by the regular lattice of fibres of radius $a+d / 2$, as shown in Figure 2.2 (b). Further details of the security-spheres approach can 
be found in (RUBENFELD AND KELlER, 1969), (TORQUATO AND RUBINSHTEIN, 1991) and (TORQUATO, 2002).
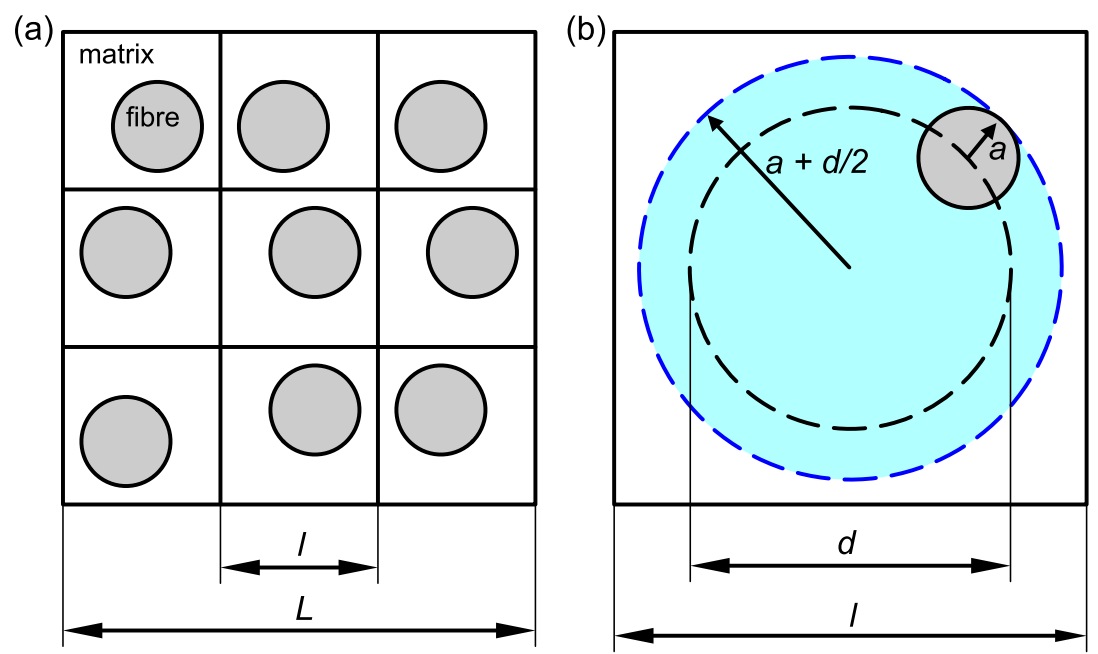

Figure 2.2: (a) Shaking-Geometry Composite, (b) deviation of the fibers about the regular square lattice.

Introducing lower $K_{1}$ and upper $K_{2}$ bounds for $\langle k\rangle$ such that $K_{1} \leqslant\langle k\rangle \leqslant K_{2}$, and let the conductivity of the perfectly regular material be denoted as a function $K_{0}$ of the fiber volume fraction $c$. Then, the lower bound $K_{1}$ is given by the solution in the perfectly regular case at $\delta=0$, (Berlyand And Mityushev, 2001), (Berlyand And Mityushev, 2005) and (KOLZLOV, 1989).

$$
K_{1}=\left.\langle k\rangle\right|_{\delta=0}
$$

For the regular case, the formulation proposed by Perrins et al. (PERRINS et al., 1979) is used. Several models for the regular case are implemented and studied by Kalakarov et al. (KALAMKAROV et al., 2009) and Andrianov et al. (ANDRIANOv et al., 2008). The analytical expression for the conductivity, as defined by (PERRINS et al., 1979), is:

$$
\left.\langle k\rangle\right|_{\delta=0}=K_{1}=\frac{1-2 c}{\left[T+c-\frac{0.305827 c^{4} T}{T^{2}-1.402958 c^{8}}-\frac{0.013362 c^{8}}{T}\right]},
$$


where

$$
T=\frac{1+k_{f} / k_{m}}{1-k_{f} / k_{m}}
$$

To obtain the upper bound $K_{2}$, the original nonregular assembly of fibers of radius $a$ is replaced by a regular lattice of fibers of radius $a+d / 2$. This estimation yields

$$
K_{2}=\left.\langle k\rangle\right|_{\delta=0}\left(c=c+2 \delta \sqrt{c c_{\max }}+\delta^{2} c_{\max }\right) .
$$

It is worth to point out that the percolation threshold is reached at the maximum volume fraction $c_{\max } \approx 0.41$, (MOLCHANOV, 1991). 


\section{BEM for 3D anisotropic elasticity}

\subsection{Introduction}

The analytical and numerical formulation of the BEM for 3D general anisotropic elasticity is reviewed in this chapter. First, a brief introduction of the general anisotropic fundamental solutions as derived by Lifshitz and Rozenzweig (1947) and, Ting and Lee (1997) is presented. Then, the Fourier series representation of the displacement fundamental solution and its derivatives, as proposed by Shiah et al. (2012a) and Tan et al. (2013), is reviewed. The multi-domain BEM approach using this latter fundamental solution is implemented. The adaptation of this multi-domain approach to model fibre-matrix problems is also discussed. Numerical examples are presented in order to test the validity of the implemented formulation.

\subsection{The 3D anisotropic fundamental solutions}

The boundary integral equation (BIE) for elastostatics relates the nodal displacements, $u_{i}$, and tractions, $t_{i}$, at the boundary $\Gamma$ of a homogeneous elastic domain in the absence of body forces can be expressed as,

$$
C_{i j} u_{i}+\int_{\Gamma} T_{i j} u_{i} d S=\int_{\Gamma} U_{i j} t_{i} d \Gamma
$$

where $i, j=1,2,3$, the value of $C_{i j}$ depends on the geometry at the source point. $U_{i j}$ and $T_{i j}$ are the fundamental solutions for displacements and tractions, respectively.

The displacement fundamental solution or Green's function, $\mathbf{U}(\mathbf{x})=U_{i j}(P, Q)$, is defined as the displacement response in the $x_{i}$ direction at the field point $Q$ due to a unit load applied in the $x_{j}$ direction at the source point $P$ in a homogeneous infinite body. This solution for the case of 3D generally anisotropic materials was first derived by Lifshitz and Rozenzweig (1947), and can be written as

$$
U_{i j}=\frac{1}{8 \pi^{2} r} \int_{0}^{2 \pi} \mathbf{Z}^{-1} d \psi
$$


where $r$ is the distance between the source and the field point. The integrand $\mathbf{Z}^{-1}$ is the inverse of the matrix $\mathbf{Z}$, that can be expressed in terms of the elastic stiffness tensor of the anisotropic material $C_{i j k s} \equiv$ C, (SHIAH et al., 2012b). Ting and Lee (1997) showed that the Green's function can be also expressed in terms of the Barnett-Lothe tensor, $\mathbf{H}[\mathbf{x}]$. In the solution first derived by Lifshitz and Rozenzweig (1947), $\mathbf{H}[\mathbf{x}]$ is expressed as a contour integral around a unit circle at the field point $Q$ on a oblique plane whose normal is the position vector $\mathbf{x}_{Q}$, as shown in Figure 3.1.

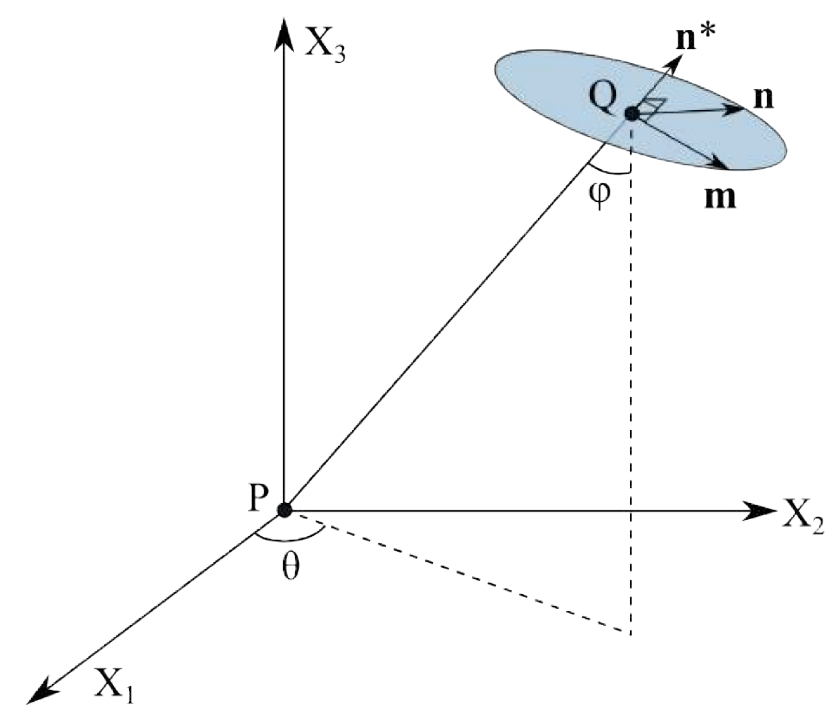

Figure 3.1: Normal vectors at the field point $Q$.

The tangential vectors $\mathbf{n}$ and $\mathbf{m}$ are mutually orthogonal unit vectors and $\left[\mathbf{n}, \mathbf{m}, \mathbf{n}^{*}\right]$ form a right-handed triad, where $\mathbf{n}^{*}=\mathbf{x}_{Q} / r$. The tangential vectors can be expressed in spherical coordinates as,

$$
\begin{aligned}
& \mathbf{n}=(\cos \phi \cos \theta, \cos \phi \sin \theta,-\sin \phi), \\
& \mathbf{m}=(-\sin \theta, \cos \theta, 0)
\end{aligned}
$$

where $0 \leq \theta<2 \pi$ and $0 \leq \phi \leq \pi$.

Consider a spherical coordinate system, the explicit form of the Green's function can also be expressed as follows, 


$$
\mathbf{U}(r, \theta, \phi)=\frac{1}{4 \pi r} \mathbf{H}(\theta, \phi)
$$

This expression depends only on the spherical angles $(\theta, \phi)$ and can be further expressed in terms of the Stroh's eigenvalues as,

$$
\mathbf{H}(\theta, \phi)=\frac{1}{|\boldsymbol{\kappa}|} \sum_{n=0}^{4} q_{n} \hat{\Gamma}^{(n)}
$$

where the quantities $q_{n}, \hat{\Gamma}^{(n)}$ and $\kappa$ are given by

$$
\begin{aligned}
& q_{n}= \begin{cases}\frac{-1}{2 \beta_{1} \beta_{2} \beta_{3}}\left[\Re\left\{\sum_{t=1}^{3} \frac{p_{t}^{n}}{\left(p_{t}-\bar{p}_{t+1}\right)\left(p_{t}-\bar{p}_{t+2}\right)}\right\}-\delta_{n 2}\right] & \text { for } n=0,1,2, \\
\frac{-1}{2 \beta_{1} \beta_{2} \beta_{3}}\left[\Re\left\{\sum_{t=1}^{3} \frac{p_{t}^{n-2} \bar{p}_{t+1} \bar{p}_{t+2}}{\left(p_{t}-\bar{p}_{t+1}\right)\left(p_{t}-\bar{p}_{t+2}\right)}\right\}\right] & \text { for } n=3,4,\end{cases} \\
& \hat{\Gamma}_{i j}^{(n)}=\widetilde{\Gamma}_{(i+1)(j+1)(i+2)(j+2)}^{(n)}-\widetilde{\Gamma}_{(i+1)(j+2)(i+2)(j+1)}^{(n)}, \quad(i, j=1,2,3), \\
& \boldsymbol{\kappa} \equiv \kappa_{i k}=C_{i j k s} m_{j} m_{s}
\end{aligned}
$$

where $C_{i j k s}$ are, as before, the elastic stiffness tensor of the anisotropic material. Additionally, $\hat{\Gamma}^{(n)}$ is the adjoint of the matrix $\Gamma^{(n)}$, which is defined as

$$
\boldsymbol{\Gamma}(p)=\mathbf{Q}+p \mathbf{V}+p^{2} \boldsymbol{\kappa}
$$

where

$$
\mathbf{V}=\left(\mathbf{R}+\mathbf{R}^{\mathrm{T}}\right)
$$


and

$$
\mathbf{Q} \equiv Q_{i k}, \quad \mathbf{R} \equiv R_{i k}=C_{i j k s} n_{j} m_{s}
$$

The Stroh's eigenvalues, $p_{i}$, are the roots of the sextic equation which is obtained from setting the determinant $|\boldsymbol{\Gamma}(p)|=0$. The roots appear as three pairs of complex conjugates and can be written as

$$
p_{v}=\alpha_{v}+\mathbf{i} \beta_{v}, \quad \beta_{v}>0, \quad(v=1,2,3),
$$

where $\alpha_{v}$ and $\beta_{v}$ are both real, and $\mathbf{i}=\sqrt{-1}$. The over-bar on $p_{i}$ denotes the corresponding conjugate. Shiah et al. (2008) have demonstrated that after some algebraic manipulation, the fourth-order tensor $\widetilde{\boldsymbol{\Gamma}}^{(n)}$ can be reduced to

$$
\begin{aligned}
& \widetilde{\Gamma}_{p q r s}^{(4)}=\kappa_{p q} \kappa_{r s}, \\
& \widetilde{\Gamma}_{p q r s}^{(3)}=V_{p q} \kappa_{r s}+\kappa_{p q} V_{r s}, \\
& \widetilde{\Gamma}_{p q r s}^{(2)}=\kappa_{p q} Q_{r s}+\kappa_{r s} Q_{p q}+V_{p q} V_{r s}, \\
& \widetilde{\Gamma}_{p q r s}^{(1)}=V_{p q} \kappa_{r s}+V_{r s} Q_{p q}, \\
& \widetilde{\Gamma}_{p q r s}^{(0)}=Q_{p q} Q_{r s} .
\end{aligned}
$$

The calculation of the anisotropic fundamental solution $U_{i j}$ is relatively simple and straightforward in its implementation; the only numerical approach required is the evaluation of the Stroh's eigenvalues from the sextic equation, Equation (3.9). The BIE for elastostatics also requires the evaluation of the traction fundamental solution, $T_{i j}$. This may be carried out by using the following relation,

$$
T_{i j}=\left(\sigma_{i k} N_{k}\right)_{j}
$$

where $\sigma_{i k}$ is the fundamental solution for stresses at a field point due to a concentrated force applied in the $x_{j}$ direction at the source point, and $N_{k}$ is the outward normal vector at the field point. Stresses $\sigma_{i k}$ can be determined using the generalized Hooke's law, 


$$
\boldsymbol{\sigma}_{j}=\mathrm{C} \varepsilon_{j}
$$

where stresses $\sigma_{j}$ and strains $\varepsilon_{l}$ may be expressed as,

$$
\begin{aligned}
& \boldsymbol{\sigma}_{l}=\left(\sigma_{11}, \sigma_{22}, \sigma_{33}, \sigma_{23}, \sigma_{13}, \sigma_{12}\right)_{l}^{\mathrm{T}}, \\
& \boldsymbol{\varepsilon}_{l}=\left(\varepsilon_{11}, \varepsilon_{22}, \varepsilon_{33}, 2 \varepsilon_{23}, 2 \varepsilon_{13}, 2 \varepsilon_{12}\right)_{l}^{\mathrm{T}} .
\end{aligned}
$$

The corresponding strain components $\left(\varepsilon_{i k}\right)_{l}$ are computed using the strain-displacement relations:

$$
\left(\varepsilon_{i k}\right)_{l}=\frac{\left(U_{i l, j}+U_{j l, i}\right)}{2}
$$

It is evident that the first derivative of the anisotropic fundamental solution is needed, $U_{i j, k}$. Moreover, the second derivative $U_{i j, k l}$ may be required for the calculation of stresses at interior points. The second derivative of the displacement fundamental solution appears when calculating the first derivative of the traction fundamental solution $T_{i j, k}$. The latter appearing when applying spatial differentiation of the Somigliana's identity at interior points.

\subsection{Fourier series representation}

The efficient and fast evaluation of $\mathbf{U}(\mathbf{x})$ and its derivatives is essential in order to maintain the BEM as a good numerical tool for treating large 3D practical engineering problems in general anisotropic elasticity. Shiah et al. (2012a) proposed an alternative and accurate numerical scheme to compute these quantities and further Tan et al. (2013) implemented them into a BEM code for stress analysis. This approach takes advantage of periodic nature of the BarnettLothe tensor, $\mathbf{H}(\theta, \phi)$, representing it by double Fourier series around $\theta$ and $\phi$, as follows,

$$
H_{u v}(\theta, \phi)=\sum_{m=-\infty}^{\infty} \sum_{n=-\infty}^{\infty} \lambda_{u v}^{(m, n)} e^{i(m \theta+n \phi)}, \quad(u, v=1,2,3)
$$

where $\alpha$ is an integer number, large enough to yield the desired accuracy. The Fourier coeffi- 
cients $\lambda_{u v}^{(m, n)}$ are given by

$$
\lambda_{u v}^{(m, n)}=\frac{1}{4 \pi^{2}} \int_{-\pi}^{\pi} \int_{-\pi}^{\pi} H_{u v}(\theta, \phi) e^{-i(m \theta+n \phi)} d \theta d \phi,
$$

The Fourier coefficients have to be determined. They can be obtained by numerical integration by, e.g., Gaussian quadrature. The $k$ abscissa points, $\lambda_{u v}^{(m, n)}$, may be re-written as

$$
\lambda_{u v}^{(m, n)}=\frac{1}{4} \sum_{p=1}^{k} \sum_{q=1}^{k} w_{p} w_{q} f_{u v}^{(m, n)}\left(\pi \xi_{p}, \pi \xi_{q}\right),
$$

where $w$ and $\xi$ are the weights and Gauss points, respectively and $f_{u v}^{(m, n)}(\theta, \phi)$ represents the integrand of $\lambda_{u v}^{(m, n)}$. In short, the fundamental displacement solution can also be written as

$$
U_{u v}(r, \theta, \phi)=\frac{1}{4 \pi r} \sum_{m=-\alpha}^{\alpha} \sum_{n=-\alpha}^{\alpha} \lambda_{u v}^{(m, n)} e^{i(m \theta+n \phi)}
$$

Numerical experiments by Tan et al. (2013) have shown that values of $k=64$ and $\alpha=16$ will be adequate to evaluate even the most highly anisotropic materials. The most significant advantage of using this Fourier series representation of the Green's function is that the Fourier series coefficients, $\lambda_{u v}^{(m, n)}$, are only evaluated once. This further reduces the computational efforts, besides the relative simplicity of the implementation into a BEM code.

A reduction of the Fourier coefficients that need to be evaluated was also accounted by Tan et al. (2013). The quantity, $\lambda_{u v}^{(m, n)}$, can be separated into real, $R_{u v}^{(m, n)}$, and imaginary parts $I_{u v}^{(m, n)}$, as follows,

$$
\lambda_{u v}^{(m, n)}=R_{u v}^{(m, n)}+\mathbf{i} I_{u v}^{(m, n)} .
$$

The imaginary part of $H_{u v}(\theta, \phi)$ must vanish. Equation (3.18) can be rewritten as 


$$
H_{u v}(\theta, \phi)=\sum_{m=-\alpha}^{\alpha} \sum_{n=-\alpha}^{\alpha} h_{u v}^{(m, n)}(\theta, \phi)
$$

where $h_{u v}^{(m, n)}(\theta, \phi)$ is defined by

$$
h_{u v}^{(m, n)}(\theta, \phi)=R_{u v}^{(m, n)} \cos m \theta+n \phi-I_{u v}^{(m, n)} \sin m \theta+n \phi .
$$

It can be noted from Equation (3.19) that $\lambda_{u v}^{(m, n)}$ and $\lambda_{u v}^{(-m,-n)}$ are complex conjugates, i.e., $\lambda_{u v}^{(-m,-n)}=\bar{\lambda}_{u v}^{(m, n)}$. Substituting into Equation (3.24), it can be reduced to

$$
\begin{aligned}
H_{u v}(\theta, \phi)= & 2 \sum_{m=1}^{\alpha}\left\{\sum_{n=1}^{\alpha} h_{u v}^{(m, n)}(\theta, \phi)+\sum_{n=-\alpha}^{-1} h_{u v}^{(m, n)}(\theta, \phi)\right\} \\
& +2 \sum_{n=1}^{\alpha} h_{u v}^{(0, n)}(\theta, \phi)+2 \sum_{m=1}^{\alpha} h_{u v}^{(m, 0)}(\theta, \phi)+R_{u v}^{(0,0)}
\end{aligned}
$$

Substituting Equation (3.24) and Equation (3.25) into Equation (3.4), the fundamental solution $\mathrm{U}$ can be expressed as

$$
U_{u v}=\frac{1}{2 \pi r}\left\{\begin{array}{c}
\sum_{m=1}^{\alpha} \sum_{n=1}^{\alpha}\left[\begin{array}{c}
\left(\widetilde{R}_{u v}^{(m, n)} \cos m \theta-\widetilde{I}_{u v}^{(m, n)} \sin m \theta\right) \cos n \phi \\
-\left(\hat{R}_{u v}^{(m, n)} \sin m \theta+\hat{I}_{u v}^{(m, n)} \cos m \theta\right) \sin n \phi
\end{array}\right] \\
+\sum_{m=1}^{\alpha}\left(\begin{array}{l}
R_{u v}^{(0, m)} \cos m \phi-I_{u v}^{(0, m)} \sin m \phi \\
+R_{u v}^{(m, 0)} \cos m \theta-I_{u v}^{(m, 0)} \sin m \theta
\end{array}\right)+\frac{R_{u v}^{(0,0)}}{2}
\end{array}\right\}
$$

where $\widetilde{R}_{u v}^{(m, n)}, \hat{R}_{u v}^{(m, n)}, \widetilde{I}_{u v}^{(m, n)}$, and $\hat{I}_{u v}^{(m, n)}$ are given by

$$
\begin{aligned}
& \widetilde{R}_{u v}^{(m, n)}=R_{u v}^{(m, n)}+R_{u v}^{(m,-n)}, \\
& \hat{R}_{u v}^{(m, n)}=R_{u v}^{(m, n)}-R_{u v}^{(m,-n)}, \\
& \widetilde{I}_{u v}^{(m, n)}=I_{u v}^{(m, n)}+I_{u v}^{(m,-n)}, \\
& \hat{I}_{u v}^{(m, n)}=I_{u v}^{(m, n)}-I_{u v}^{(m,-n)} .
\end{aligned}
$$




\subsection{Derivatives of the anisotropic fundamental solution}

Lee (2009), using partial differentiations of the Green's function in spherical coordinates, eliminated the necessity of working with high order tensors, as those seen in (TING AND LEE, 1997) and (LEE, 2003). The displacement derivatives can be obtained by working in spherical coordinates and applying the chain rule. All explicit forms are given in (SHIAH et al.; SHIAH et al., 2010; 2012b) and (TAN et al., 2013). The displacement derivatives can be written in spherical coordinates as,

$$
U_{i j, l}=\frac{\partial U_{i j}}{\partial r} \frac{\partial r}{\partial x_{l}}+\frac{\partial U_{i j}}{\partial \theta} \frac{\partial \theta}{\partial x_{l}}+\frac{\partial U_{i j}}{\partial \phi} \frac{\partial \phi}{\partial x_{l}}
$$

The partial derivatives of $U_{i j}$ with respect to the spherical coordinates $r, \theta$ and $\phi$ can be obtained as

$$
\frac{\partial U_{i j}}{\partial r}=\frac{-U_{i j}}{r}, \quad \frac{\partial U_{i j}}{\partial \theta}=\frac{I_{1}-J_{1}}{4 \pi^{2} r}, \quad \frac{\partial U_{i j}}{\partial \phi}=\frac{I_{2}-J_{2}}{4 \pi^{2} r}
$$

where the closed-form expressions of $I_{1}, I_{2}, J_{1}$ and $J_{2}$ are given in (LEE, 2009). Differentiating once again Equation (3.28) and using Equation (3.29), we have,

$$
\begin{gathered}
\frac{\partial^{2} U_{i j}}{\partial r^{2}}=\frac{U_{i j}}{r^{2}}-\frac{\partial U_{i j}}{\partial r} \quad \frac{\partial^{2} U_{i j}}{\partial r \partial \theta}=-\frac{1}{r^{2}} \frac{\partial U_{i j}}{\partial \theta} \quad \frac{\partial^{2} U_{i j}}{\partial r \partial \phi}=-\frac{1}{r^{2}} \frac{\partial U_{i j}}{\partial \phi} \\
\frac{\partial^{2} U_{i j}}{\partial \theta^{2}}=\frac{1}{4 \pi^{2} r}\left(\frac{\partial I_{i j}^{\prime}}{\partial \theta}-\frac{\partial J_{i j}^{\prime}}{\partial \theta}\right), \quad \frac{\partial^{2} U_{i j}}{\partial \phi^{2}}=\frac{1}{4 \pi^{2} r}\left(\frac{\partial I^{\prime \prime}{ }_{i j}}{\partial \phi}-\frac{\partial{J^{\prime \prime}}_{i j}}{\partial \phi}\right), \\
\frac{\partial^{2} U_{i j}}{\partial \theta \partial \phi}=\frac{1}{4 \pi^{2} r}\left(\frac{\partial I^{\prime \prime}{ }_{i j}}{\partial \theta}-\frac{\partial J^{\prime \prime}{ }_{i j}}{\partial \theta}\right)
\end{gathered}
$$

Components of Equation (3.31) are expressed in terms of the Stroh's eigenvalues and their closed-forms are given in (SHIAH et al.; TAN et al., 2010; 2013). Partial differentiations are carried out on the Fourier series. Substituting Equation (3.20) in Equation (3.28) yields 


$$
U_{i j, l}=\frac{1}{4 \pi r^{2}}\left\{\begin{array}{cc}
\sum_{m=-\alpha}^{\alpha} \sum_{n=-\alpha}^{\alpha} \lambda_{i j}^{(m, n)} e^{i(m \theta+n \phi)}\left[\begin{array}{c}
-\cos \theta(\sin \phi-i n \cos \phi) \\
-i m \sin \theta / \sin \phi
\end{array}\right] & \text { for } l=1 \\
\sum_{m=-\alpha}^{\alpha} \sum_{n=-\alpha}^{\alpha} \lambda_{i j}^{(m, n)} e^{i(m \theta+n \phi)}\left[\begin{array}{c}
-\sin \theta(\sin \phi-i n \cos \phi) \\
+i m \cos \theta / \sin \phi
\end{array}\right] & \text { for } l=2 \\
\sum_{m=-\alpha}^{\alpha} \sum_{n=-\alpha}^{\alpha} \lambda_{i j}^{(m, n)} e^{i(m \theta+n \phi)}[-(\cos \phi+i n \sin \phi)] & \text { for } l=3
\end{array}\right.
$$

However, the re-formulated form of $\mathbf{U}$, Equation (3.26), can be applied into Equation (3.28) to also obtain a reduction on the number of terms of $\mathbf{U}^{\prime}$. Letting $\omega_{l}(\theta, \phi), \omega_{l}^{\prime}(\theta, \phi)$ and $\omega_{l}^{\prime \prime}(\theta, \phi)$ to represent spatial differentiations as,

$$
\begin{gathered}
\omega_{l}(\theta, \phi)=r \frac{\partial r}{\partial x_{l}}= \begin{cases}\sin \phi \cos \theta, & (\text { for } l=1), \\
\sin \phi \sin \theta, & (\text { for } l=2), \\
\cos \phi, & (\text { for } l=3),\end{cases} \\
\omega_{l}^{\prime}(\theta, \phi)=r \frac{\partial \theta}{\partial x_{l}}= \begin{cases}-\sin \theta / \sin \phi, & (\text { for } l=1), \\
\cos \theta \sin \phi, & (\text { for } l=2), \\
0, & (\text { for } l=3),\end{cases} \\
\omega_{l}^{\prime \prime}(\theta, \phi)=r \frac{\partial \phi}{\partial x_{l}}= \begin{cases}\cos \phi \cos \theta, & (\text { for } l=1), \\
\cos \phi \sin \theta, & (\text { for } l=2), \\
-\sin \phi, & (\text { for } l=3),\end{cases}
\end{gathered}
$$

Thus, the Fourier series expression for the first derivative $\mathbf{U}^{\prime}$ can be represented by 


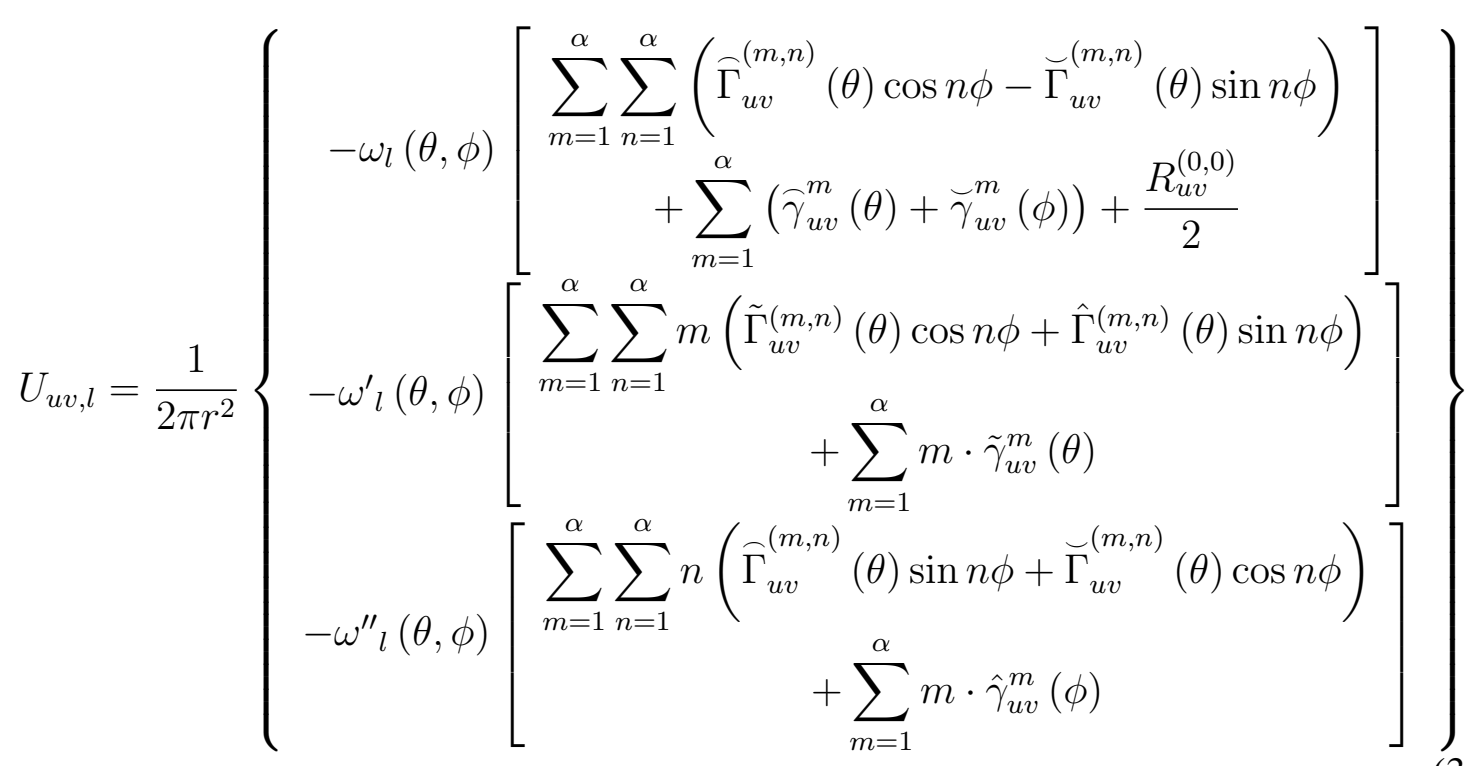

where

$$
\begin{aligned}
& \hat{\Gamma}_{u v}^{(m, n)}(\theta)=\widetilde{R}_{u v}^{(m, n)} \cos m \theta-\widetilde{I}_{u v}^{(m, n)} \sin m \theta, \\
& \breve{\Gamma}_{u v}^{(m, n)}(\theta)=\hat{R}_{u v}^{(m, n)} \sin m \theta+\hat{I}_{u v}^{(m, n)} \cos m \theta, \\
& \widetilde{\Gamma}_{u v}^{(m, n)}(\theta)=\widetilde{R}_{u v}^{(m, n)} \sin m \theta+\widetilde{I}_{u v}^{(m, n)} \cos m \theta, \\
& \hat{\Gamma}_{u v}^{(m, n)}(\theta)=\hat{R}_{u v}^{(m, n)} \cos m \theta-\hat{I}_{u v}^{(m, n)} \sin m \theta, \\
& \hat{\gamma}_{u v}^{m}(\theta)=R_{u v}^{(m, 0)} \cos m \theta-I_{u v}^{(m, 0)} \sin m \theta, \\
& \breve{\gamma}_{u v}^{m}(\phi)=R_{u v}^{(0, m)} \cos m \phi-I_{u v}^{(0, m)} \sin m \phi, \\
& \widetilde{\gamma}_{u v}^{m}(\theta)=R_{u v}^{(m, 0)} \sin m \theta+I_{u v}^{(m, 0)} \cos m \theta, \\
& \hat{\gamma}_{u v}^{m}(\phi)=R_{u v}^{(0, m)} \sin m \phi+I_{u v}^{(0, m)} \cos m \phi,
\end{aligned}
$$

As well as in the calculation of $\mathbf{U}$, Equation (3.26), the series sum interval has been reduced to half of the original. Additionally, many of the calculated coefficients are small when compared to others in the series and can be disregarded from the summation. For some cases, as high as $90 \%$ of the coefficients can be disregarded without any further loss of accuracy when finding $\mathbf{U}$ and $\mathbf{U}^{\prime}$.

Similarly, $2^{\text {nd }}$ order derivatives can be obtained by applying the chain rule in the spherical coordinate system and working with reduced expressions of the Fourier series. Due to limitation of space, expressions for the second derivatives will not be presented here. However, all explicit 
forms are available in (SHIAH et al.; TAN et al., 2010; 2013).

\subsection{Discretization of the BIE}

The BIE given in Equation (3.1) can be discretized using some appropriate type of element, as follows, (Kane, 1994),

$$
C_{i k} u_{i}+\sum_{E=1}^{N E L} \int_{\Gamma_{E}} t_{i k}\left(\sum_{N=1}^{N N E L} h^{(N)} u_{i}^{(N)}\right) \mathrm{d} \Gamma=\sum_{E=1}^{N E L} \int_{\Gamma_{E}} u_{i k}\left(\sum_{N=1}^{N N E L} h^{(N)} t_{i}^{(N)}\right) \mathrm{d} \Gamma
$$

or

$$
\begin{aligned}
C_{i k} u_{i}+ & \sum_{E=1}^{N E L}\left(\sum_{N=1}^{N N E L} \int_{-1}^{+1} \int_{-1}^{+1} t_{i k} h^{(N)} g \mathrm{~d} \xi \mathrm{d} \eta\right)^{(E)} u_{i}^{(N)} \\
= & \sum_{E=1}^{N E L}\left(\sum_{N=1}^{N N E L} \int_{-1}^{+1} \int_{-1}^{+1} u_{i k} h^{(N)} g \mathrm{~d} \xi \mathrm{d} \eta\right)^{(E)} t_{i}^{(N)}
\end{aligned}
$$

where $N E L$ is the number of elements, $N$ is the node number of the analysed element $E$ and $N N E L$ is the total number of nodes in the element $E$. The shape functions are represented by $h^{(N)}$ and are dependent of the parametric position of the node $(\xi, \eta)$.

In the present work, the eight-node discontinuous quadratic element is considered, as can be seen in Figure 3.2. 


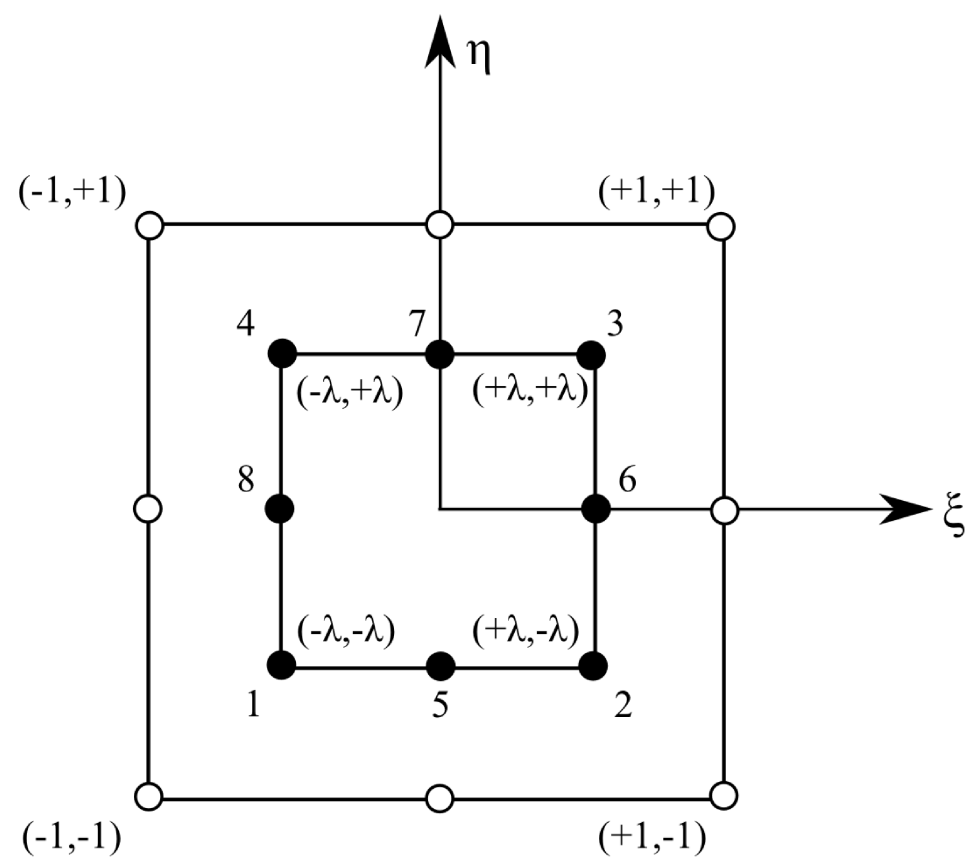

Figure 3.2: (a) Eight-node discontinuous quadratic element.

The surface response are interpolated within an element from nodal values using shape functions. The shape functions for the eight-node quadratic discontinuous element are:

$$
\begin{aligned}
h^{(1)} & =\frac{1}{4 \lambda^{3}}(\lambda-\xi)(\lambda-\eta)(-\xi-\eta-\lambda) \\
h^{(2)} & =\frac{1}{4 \lambda^{3}}(\lambda+\xi)(\lambda-\eta)(\xi-\eta-\lambda) \\
h^{(3)} & =\frac{1}{4 \lambda^{3}}(\lambda+\xi)(\lambda+\eta)(\xi+\eta-\lambda) \\
h^{(4)} & =\frac{1}{4 \lambda^{3}}(\lambda-\xi)(\lambda+\eta)(-\xi+\eta-\lambda) \\
h^{(5)} & =\frac{1}{2 \lambda^{3}}\left(\lambda^{2}-\xi^{2}\right)(\lambda-\eta) \\
h^{(6)} & =\frac{1}{2 \lambda^{3}}\left(\lambda^{2}-\eta^{2}\right)(\lambda+\xi) \\
h^{(7)} & =\frac{1}{2 \lambda^{3}}\left(\lambda^{2}-\xi^{2}\right)(\lambda+\eta) \\
h^{(8)} & =\frac{1}{2 \lambda^{3}}\left(\lambda^{2}-\eta^{2}\right)(\lambda-\xi)
\end{aligned}
$$

where $\lambda$ is the discontinuous parameter of the element, as can be seen in Figure 3.2. 
The expression in Equation (3.57) can be represented in matrix form as,

$$
\sum_{E=1}^{N E L}[F]^{(\mathbf{d}, E)}\{u\}^{E}=\sum_{E=1}^{N E L}[G]^{(\mathbf{d}, E)}\{t\}^{E}
$$

where

$$
\begin{aligned}
& {[F]^{(\mathbf{d}, E)}=\int_{-1}^{+1} \int_{-1}^{+1}[T]^{\mathrm{T}}[H] g \mathrm{~d} \xi \mathrm{d} \eta} \\
& {[G]^{(\mathbf{d}, E)}=\int_{-1}^{+1} \int_{-1}^{+1}[U]^{\mathrm{T}}[H] g \mathrm{~d} \xi \mathrm{d} \eta}
\end{aligned}
$$

and

$$
\begin{aligned}
& {[T]^{\mathrm{T}}=\left[\begin{array}{ccc}
t_{11} & t_{21} & t_{31} \\
t_{12} & t_{22} & t_{32} \\
t_{13} & t_{23} & t_{33}
\end{array}\right] ; \quad[U]^{\mathrm{T}}=\left[\begin{array}{lll}
u_{11} & u_{21} & u_{31} \\
u_{12} & u_{22} & u_{32} \\
u_{13} & u_{23} & u_{33}
\end{array}\right]} \\
& {[H]^{\mathrm{T}}=\left[\begin{array}{cccccccccc}
h^{(1)} & 0 & 0 & h^{(2)} & 0 & 0 & & h^{(8)} & 0 & 0 \\
0 & h^{(1)} & 0 & 0 & h^{(2)} & 0 & \cdots & 0 & h^{(8)} & 0 \\
0 & 0 & h^{(1)} & 0 & 0 & h^{(2)} & & 0 & 0 & h^{(8)}
\end{array}\right]} \\
& \{u\}^{(E)}=\left[\begin{array}{llllllllll}
u_{1}^{(1)} & u_{2}^{(1)} & u_{3}^{(1)} & u_{1}^{(2)} & u_{2}^{(2)} & u_{3}^{(2)} & \cdots & u_{1}^{(8)} & u_{2}^{(8)} & u_{3}^{(8)}
\end{array}\right]^{\mathrm{T}} \\
& \{t\}^{(E)}=\left[\begin{array}{llllllllll}
t_{1}^{(1)} & t_{2}^{(1)} & t_{3}^{(1)} & t_{1}^{(2)} & t_{2}^{(2)} & t_{3}^{(2)} & \cdots & t_{1}^{(8)} & t_{2}^{(8)} & t_{3}^{(8)}
\end{array}\right]^{\mathrm{T}}
\end{aligned}
$$

The entries for $[U]$ and $[T]$ are quantities involving the components of the fundamental solutions of displacements and tractions, respectively. The values of the integrals of Equation (3.42) can be numerically evaluated using Gaussian quadrature. The information of the integration points such as coordinates, weights, isoparametric shape functions and its derivatives are computed a priori and re-used by means of the reusable intrinsic sample point (RISP) algorithm. The RISP algorithm and the integration scheme used in this work are described in Kane (1994). Equation (3.39) represents a set of linear algebraic equations which can be solved, after 
the appropriate boundary conditions are applied, by, e.g., Gaussian elimination.

\subsection{Multi-domain analysis for fiber-matrix problems}

The multi-domain approach is first reviewed. The BIE, shown in Equation (3.1) and later in its discretized form in Equation (3.56), govern the behavior of any portion of the analysed body. This allows the development of multiple boundary integral relations that are associated with portions of overall body, as shown in Figure 3.3, (KANE, 1994).

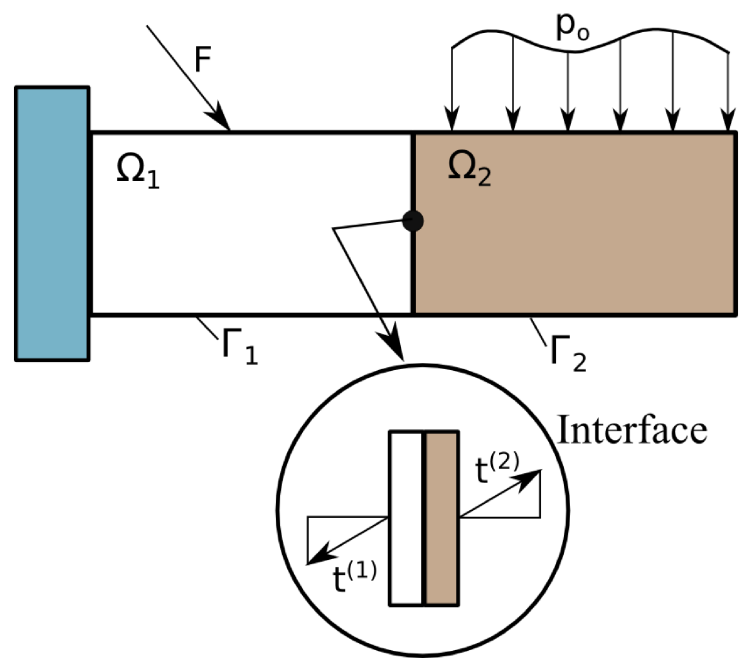

Figure 3.3: Multi-domain 2D example with two sub-regions.

The corresponding BIEs for the two sub-regions model can be represented as,

$$
\begin{aligned}
& C_{i j} u_{i}+\int_{\Gamma_{1}} T_{i j} u_{i} \mathrm{~d} \Gamma_{1}=\int_{\Gamma_{1}} U_{i j} t_{i} \mathrm{~d} \Gamma_{1}, \\
& C_{i j} u_{i}+\int_{\Gamma_{2}} T_{i j} u_{i} \mathrm{~d} \Gamma_{2}=\int_{\Gamma_{2}} U_{i j} t_{i} \mathrm{~d} \Gamma_{2},
\end{aligned}
$$

The resulting linear equations from the discretized BIEs can also be expressed as,

$$
\begin{aligned}
& {\left[F^{1}\right]\left\{u^{1}\right\}=\left[G^{1}\right]\left\{t^{1}\right\}} \\
& {\left[F^{2}\right]\left\{u^{2}\right\}=\left[G^{2}\right]\left\{t^{2}\right\}}
\end{aligned}
$$


After the BEM is applied to each sub-region, or sub-domain, the system coupling can be formulated by considering displacement compatibility and traction equilibrium at the interfaces between sub-regions. The compatibility condition requires that nodal displacements for a region $i$, at an interface between region $i$ and region $j$, must be equal to the displacement components evaluated in region $j$ at the same interface. The equilibrium between traction vectors at the interface nodes must be also considered. These conditions can be represented as,

$$
\begin{gathered}
u_{i j}^{i}=u_{i j}^{j} \\
t_{i j}^{i}=-t_{i j}^{j}
\end{gathered}
$$

where the double subscripts is used to denote the vector in question is a collection of components on the interface between region $i$ and region $j$.

Finally, applying the displacement compatibility and the traction equilibrium at the interface, the following matrix equation system can be obtained,

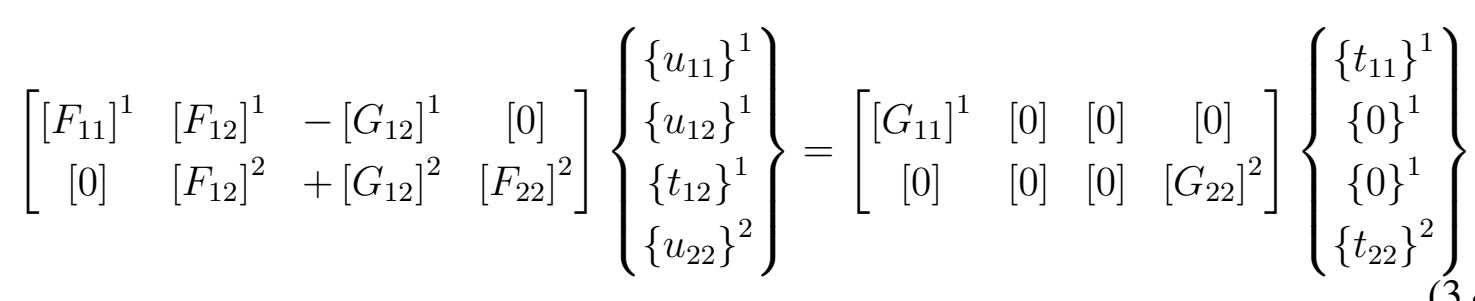

where the sub-indices 12 represent the evaluated sub-matrices $[F]$ or $[G]$ at the interface between regions 1 and 2. The sub-indices 11 and 22 represent the evaluated sub-matrices for regions 1 and 2, respectively. Further details on the multi-domain approach can be found in (KANE, 1994).

The multi-domain BEM approach is a natural choice when solving fiber-matrix problems, due to the possibility of analysing each region individually. Figure 3.4 shows a small example with four fibers, analysed here in order to illustrate the implementation. 


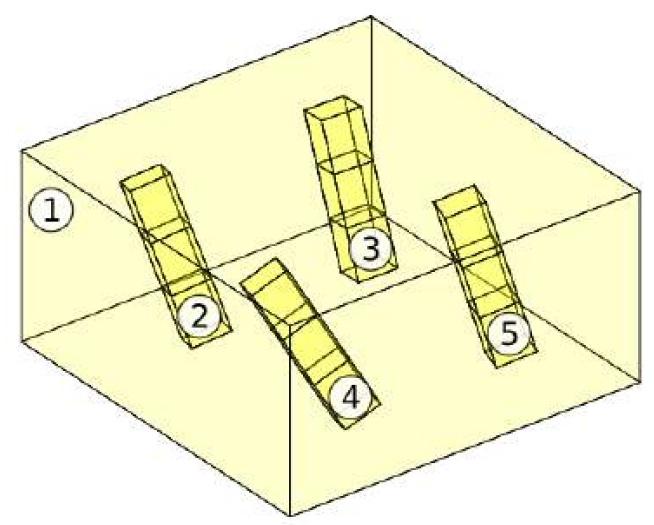

Figure 3.4: Multi-domain example with four fibers.

Five sub-regions were created for this example, namely, the matrix and the four fibers. The multi-domain collocation matrix, $\left[H_{c o l l}\right]$, generated may be written as follows:

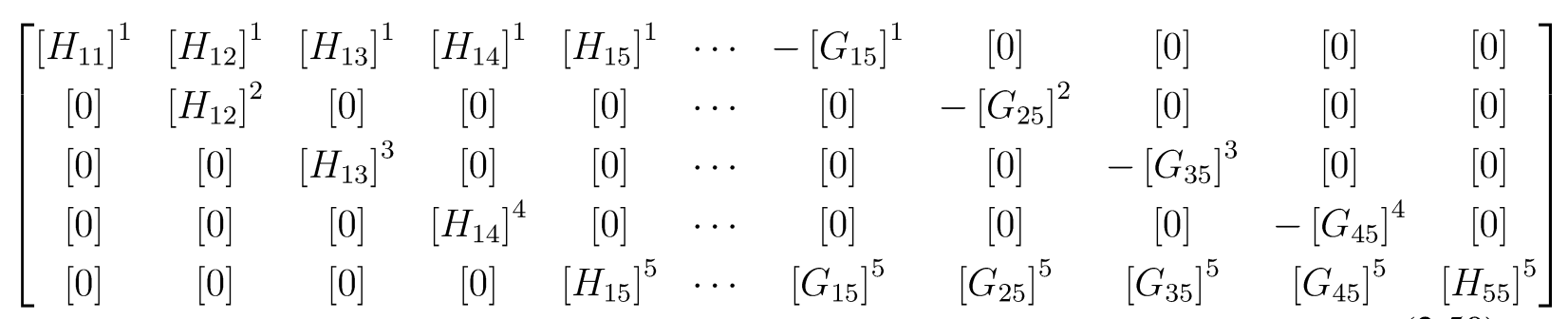

where $\left[H_{i j}\right]^{e}$ and $\left[G_{i j}\right]^{e}$ represent the incidences in matrices $[H]$ and $[G]$ between sub-regions $i$ and $j$ in the case where quantities are associated to a specific region $e$. The order of the subregion incidences are determined by first simply listing all the permutations, (KANE, 1994), for this example,

$$
\begin{array}{llllllllllllllll}
11 & 12 & 13 & 14 & 15 & 21^{*} & 22 & 23 & 24 & 25 & \cdots & 51^{*} & 52^{*} & 53^{*} & 54^{*} & 55
\end{array}
$$

where the digits represent the subregions being analysed. For the permutation where the first digit is higher than the second digit $\left(^{*}\right)$, blocks of traction components, $\left[G_{i j}\right]^{e}$, are created; otherwise, blocks of displacement components, $\left[H_{i j}\right]^{e}$, are created. The negative sign is due to opposite directions of the traction vector when analysing different regions at the interface. Since 
there is no contact among fibers here, the matrices with both indices $i$ and $j$ corresponding to fibers are null. Hence, the system can be further compressed as,

$$
\left[H_{\text {coll }}\right]=[[A], \quad[B], \quad[C]]
$$

where,

$$
\begin{aligned}
& {[A]=\left[\begin{array}{ccccc}
{\left[H_{11}\right]^{1}} & {\left[H_{12}\right]^{1}} & {\left[H_{13}\right]^{1}} & {\left[H_{14}\right]^{1}} & {\left[H_{15}\right]^{1}} \\
{[0]} & {\left[H_{12}\right]^{2}} & {[0]} & {[0]} & {[0]} \\
{[0]} & {[0]} & {\left[H_{13}\right]^{3}} & {[0]} & {[0]} \\
{[0]} & {[0]} & {[0]} & {\left[H_{14}\right]^{4}} & {[0]} \\
{[0]} & {[0]} & {[0]} & {[0]} & {\left[H_{15}\right]^{5}}
\end{array}\right]} \\
& {[B]=\left[\begin{array}{cccc}
{[0]} & {[0]} & {[0]} & {[0]} \\
{\left[H_{22}\right]^{2}} & {[0]} & {[0]} & {[0]} \\
{[0]} & {\left[H_{33}\right]^{3}} & {[0]} & {[0]} \\
{[0]} & {[0]} & {\left[H_{44}\right]^{4}} & {[0]} \\
{[0]} & {[0]} & {[0]} & {\left[H_{55}\right]^{5}}
\end{array}\right]} \\
& {[C]=\left[\begin{array}{cccc}
-\left[G_{12}\right]^{1} & -\left[G_{13}\right]^{1} & -\left[G_{14}\right]^{1} & -\left[G_{15}\right]^{1} \\
{\left[G_{12}\right]^{2}} & {[0]} & {[0]} & {[0]} \\
{[0]} & {\left[G_{13}\right]^{3}} & {[0]} & {[0]} \\
{[0]} & {[0]} & {\left[G_{14}\right]^{4}} & {[0]} \\
{[0]} & {[0]} & {[0]} & {\left[G_{15}\right]^{5}}
\end{array}\right] .}
\end{aligned}
$$

The sub-matrices, $\left[H_{i j}\right]^{e}$ and $\left[G_{i j}\right]^{e}$, are obtained applying the BEM to each $e$ region individually. The Adaptive Cross Approximation (ACA) technique can also be used to this end. It is worth to point out that, usually, the fibers have a very small number of elements and consequently the ACA would not present any advantage on the approximation. Thus, the ACA will be applied to the region which contains the matrix medium. For regular multi-domain problems no compression can be made and the system of Equation (3.50) should be used instead. 


\subsection{Numerical examples}

Five numerical examples are presented to demonstrate the successful implementation of the BEM with the Fourier series form of the fundamental solutions discussed earlier above. In the first example, the anisotropic fundamental solution based on Fourier series and its derivatives are validated with solutions presented by Ting and Lee (1997) and Shiah et al. (2012a). A generally anisotropic material is used to this end. The 3D anisotropic fundamental solution and derivatives have been implemented into a BEM code. This BEM code is tested first with an isotropic material but using the algorithm for anisotropy, Example B; then, with alpha-quartz crystal (trigonal system), Example C; and with a generally anisotropic material, Example D. In the latter example the fully populated stiffness matrix is obtained by successively rotation of the material principal axes. Finally, in Example E, the multi-domain approach is tested with four regions. In this example, an isotropic material is considered in order to properly compare the results with the analytical solution. However, the analysis was carried out using the algorithm for general anisotropy.

\subsubsection{Example A: Validation of the anisotropic fundamental solution and its derivatives}

In this example the anisotropic displacement fundamental solution and its derivatives are compared with the solution presented by Ting and Lee (1997) and Shiah et al. (2012a). The material chosen is $\mathrm{Al}_{2} \mathrm{O}_{3}$ and it has the following non-zero stiffness coefficients, (Huntington, 1958):

$$
\begin{array}{lll}
C_{11}=465 \mathrm{GPa}, & C_{33}=563 \mathrm{GPa}, & C_{44}=233 \mathrm{GPa}, \\
C_{12}=124 \mathrm{GPa}, & C_{13}=117 \mathrm{GPa}, & C_{14}=101 \mathrm{GPa},
\end{array}
$$

In order to test a fully anisotropic material the principal material axes of the $\mathrm{Al}_{2} \mathrm{O}_{3}$ crystal are successively rotated about the global Cartesian $x_{1}, x_{2}$ and $x_{3}$ directions by $30^{\circ}, 45^{\circ}$ and $60^{\circ}$ clockwise, respectively. These successive rotations yield a fully populated stiffness matrix, as follows, 


$$
\mathbf{C}=\left[\begin{array}{cccccc}
544.8 & 153.6 & 57.3 & 10.5 & 65.7 & -81.2 \\
153.6 & 531.1 & 28.4 & -14.7 & -18.1 & 89.7 \\
57.3 & 28.4 & 654.4 & 19.8 & -6.4 & 10.4 \\
10.5 & -14.7 & 19.8 & 106.4 & 24.8 & 13.3 \\
65.7 & -18.1 & -6.4 & 24.8 & 167.9 & 22.5 \\
-81.2 & 89.7 & 10.4 & 13.3 & 22.5 & 243.5
\end{array}\right] \mathrm{GPa}
$$

The source point is locate at the origin of the coordinate system. The field point, where the solutions are compared, is located at the spherical coordinates $(r=1.0, \theta=\pi / 3, \phi=\pi / 4)$.

Table 3.1 shows the numerical components of the anisotropic fundamental solution $U_{i j}$ at the field point $(r=1.0, \theta=\pi / 3, \phi=\pi / 4)$. The solution presented by Ting and Lee (1997) is used for comparison. It can be seen from Table 3.1 that a good accuracy is obtained even with $\alpha=10$; and there is excellent accuracy when $\alpha=20$, the maximum absolute difference for this case is $4.69 \times 10^{-3} \%$, for $U_{12}$.

Table 3.1: Computed values of the Green's function $U_{i j}$ at $(r=1.0, \theta=\pi / 3, \phi=\pi / 4)$.

\begin{tabular}{ccccc}
\hline$U_{i j}(i, j)$ & Ting \& Lee (1997) & $\alpha=10$ & $\alpha=15$ & $\alpha=20$ \\
\hline$(1,1)$ & 0.336396 & 0.336431 & 0.336390 & 0.336395 \\
$\mid$ Diff. $\% \mid$ & - & $1.04 \mathrm{E}-02$ & $1.78 \mathrm{E}-03$ & $2.97 \mathrm{E}-04$ \\
$(1,2)$ & 0.021313 & 0.021433 & 0.021299 & 0.021314 \\
$\mid$ Diff. $\% \mid$ & - & $5.63 \mathrm{E}-01$ & $6.57 \mathrm{E}-02$ & $4.69 \mathrm{E}-03$ \\
$(1,3)$ & -0.006535 & -0.006627 & -0.006522 & -0.006535 \\
$\mid$ Diff. $\% \mid$ & - & $-1.41 \mathrm{E}+00$ & $-1.99 \mathrm{E}-01$ & $0.00 \mathrm{E}+00$ \\
$(2,2)$ & 0.364307 & 0.364747 & 0.364252 & 0.364306 \\
$\mid$ Diff. \% & - & $1.21 \mathrm{E}-01$ & $1.51 \mathrm{E}-02$ & $2.74 \mathrm{E}-04$ \\
$(2,3)$ & -0.008461 & -0.008409 & -0.008468 & -0.008460 \\
$\mid$ Diff. \% & - & $6.15 \mathrm{E}-01$ & $8.27 \mathrm{E}-02$ & $1.18 \mathrm{E}-02$ \\
$(3,3)$ & 0.375787 & 0.376262 & 0.375729 & 0.375790 \\
$\mid$ Diff. $\% \mid$ & - & $1.26 \mathrm{E}-01$ & $1.54 \mathrm{E}-02$ & $7.98 \mathrm{E}-04$ \\
\hline
\end{tabular}

Table 3.2 shows the numerical components of the first derivative of the anisotropic fundamental solution $U_{i j, l}$. The solution presented by Shiah et al. (2012a) is used for comparison. For the Fourier series approximation a value of $\alpha=20$ is used. Great accuracy is also achieved for the first derivative; the maximum absolute difference is $6.72 \times 10^{-3} \%$ for $U_{22,1}$. 
Table 3.2: Computed first order derivative, $U_{i j, l}$, at $(r=1.0, \theta=\pi / 3, \phi=\pi / 4)$.

\begin{tabular}{|c|c|c|c|c|}
\hline \multicolumn{2}{|c|}{$U_{i j, l}(i, j)$} & \multirow{2}{*}{$\begin{array}{c}\text { Shiah et al. (2012) } \\
-0.046003\end{array}$} & $\begin{array}{c}\text { Fourier Series } \\
\quad \alpha=20\end{array}$ & \multirow{2}{*}{$\begin{array}{l}\mid \text { Diff. \% } \mid \\
4.35 \mathrm{E}-03\end{array}$} \\
\hline \multirow{6}{*}{$1=1$} & $(1,1)$ & & -0.046005 & \\
\hline & $(1,2)$ & 0.066954 & 0.066954 & $0.00 \mathrm{E}+00$ \\
\hline & $(1,3)$ & 0.075717 & 0.075717 & $0.00 \mathrm{E}+00$ \\
\hline & $(2,2)$ & -0.014878 & -0.014879 & $6.72 \mathrm{E}-03$ \\
\hline & $(2,3)$ & 0.052830 & 0.052830 & $0.00 \mathrm{E}+00$ \\
\hline & $(3,3)$ & -0.141984 & -0.141987 & $2.11 \mathrm{E}-03$ \\
\hline \multirow{6}{*}{$1=2$} & $(1,1)$ & -0.216084 & -0.216083 & $4.63 \mathrm{E}-04$ \\
\hline & $(1,2)$ & 0.021511 & 0.021511 & $0.00 \mathrm{E}+00$ \\
\hline & $(1,3)$ & -0.019238 & -0.019238 & $0.00 \mathrm{E}+00$ \\
\hline & $(2,2)$ & -0.116196 & -0.116194 & $1.72 \mathrm{E}-03$ \\
\hline & $(2,3)$ & -0.034654 & -0.034654 & $0.00 \mathrm{E}+00$ \\
\hline & $(3,3)$ & -0.429531 & -0.429530 & 2.33E-04 \\
\hline \multirow{6}{*}{$1=3$} & $(1,1)$ & -0.265599 & -0.265599 & $0.00 \mathrm{E}+00$ \\
\hline & $(1,2)$ & -0.082248 & -0.082248 & $0.00 \mathrm{E}+00$ \\
\hline & $(1,3)$ & -0.011956 & -0.011956 & $0.00 \mathrm{E}+00$ \\
\hline & $(2,2)$ & -0.407140 & -0.407140 & $0.00 \mathrm{E}+00$ \\
\hline & $(2,3)$ & 0.015561 & 0.015561 & $0.00 \mathrm{E}+00$ \\
\hline & $(3,3)$ & -0.088471 & -0.088471 & $0.00 \mathrm{E}+00$ \\
\hline
\end{tabular}

Finally, Table 3.3 shows the numerical values of the second derivative of the anisotropic fundamental solution $U_{i j, l k}$. The solution using finite differences and by Fourier series, both presented by Shiah et al. (2012a), are used for comparison. For the Fourier series approximation, a value of $\alpha=20$ is used. Good accuracy is still observed, although being the second derivative, it is less accurate than before, as was indeed expected. The maximum absolute difference when the result was compared with finite differences (F.D.) was $0.539 \%$ for $U_{23,33}$, while the maximum absolute difference when compared with the Fourier series approximation of Shiah et al. (2012a) was $0.0169 \%$ for $U_{12,23}$. 
Table 3.3: Computed second order derivatives, $U_{i j, l k}$, at $(r=1.0, \theta=\pi / 3, \phi=\pi / 4)$.

\begin{tabular}{|c|c|c|c|c|c|c|}
\hline \multirow{2}{*}{\multicolumn{2}{|c|}{$U_{i j, l k}(i, j)$}} & \multirow[b]{2}{*}{ Finite Differences } & \multirow[b]{2}{*}{ Shiah et al (2012) } & \multirow[b]{2}{*}{ Present } & \multicolumn{2}{|c|}{ 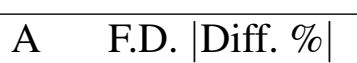 } \\
\hline & & & & & $\mathrm{B}$ & Fourier |Diff. \%| \\
\hline \multirow{6}{*}{$1 \mathrm{k}=11$} & $(1,1)$ & -0.151482 & -0.151511 & -0.151467 & $\mathrm{~A}$ & $9.90 \mathrm{E}-03$ \\
\hline & $(1,2)$ & -0.172021 & -0.172278 & -0.172273 & B & $2.90 \mathrm{E}-03$ \\
\hline & $(1,3)$ & -0.092932 & -0.092992 & -0.092996 & A & $6.89 \mathrm{E}-02$ \\
\hline & $(2,2)$ & -0.390608 & -0.391084 & -0.391029 & $\mathrm{~B}$ & $1.41 \mathrm{E}-02$ \\
\hline & $(2,3)$ & 0.019137 & 0.019072 & 0.019067 & A & $3.66 \mathrm{E}-01$ \\
\hline & $(3,3)$ & -0.339161 & -0.338994 & -0.338940 & $\mathrm{~B}$ & $1.59 \mathrm{E}-02$ \\
\hline \multirow{6}{*}{$\mathrm{lk}=12$} & $(1,1)$ & 0.047583 & 0.047548 & 0.047529 & $\mathrm{~A}$ & $1.13 \mathrm{E}-01$ \\
\hline & $(1,2)$ & 0.045261 & 0.045218 & 0.045215 & $\mathrm{~B}$ & $6.63 \mathrm{E}-03$ \\
\hline & $(1,3)$ & -0.173575 & -0.173608 & -0.173606 & A & $1.79 \mathrm{E}-02$ \\
\hline & $(2,2)$ & 0.155377 & 0.155318 & 0.155294 & $\mathrm{~B}$ & $1.55 \mathrm{E}-02$ \\
\hline & $(2,3)$ & -0.065790 & -0.065840 & -0.065837 & A & $7.14 \mathrm{E}-02$ \\
\hline & $(3,3)$ & 0.538232 & 0.538167 & 0.538140 & $\mathrm{~B}$ & $5.02 \mathrm{E}-03$ \\
\hline \multirow{6}{*}{$\mathrm{lk}=13$} & $(1,1)$ & 0.164674 & 0.164693 & 0.164693 & $\mathrm{~A}$ & $1.15 \mathrm{E}-02$ \\
\hline & $(1,2)$ & -0.142523 & -0.142395 & -0.142395 & B & $0.00 \mathrm{E}+00$ \\
\hline & $(1,3)$ & -0.017374 & -0.017316 & -0.017316 & A & $3.34 \mathrm{E}-01$ \\
\hline & $(2,2)$ & 0.102947 & 0.103112 & 0.103111 & B & $9.70 \mathrm{E}-04$ \\
\hline & $(2,3)$ & -0.102038 & -0.101943 & -0.101943 & A & $9.31 \mathrm{E}-02$ \\
\hline & $(3,3)$ & 0.104942 & 0.105023 & 0.105026 & B & $2.86 \mathrm{E}-03$ \\
\hline \multirow{6}{*}{$1 \mathrm{k}=22$} & $(1,1)$ & -0.003451 & -0.003365 & -0.003365 & $\mathrm{~A}$ & $2.49 \mathrm{E}+00$ \\
\hline & $(1,2)$ & -0.082709 & -0.082685 & -0.082681 & B & 4.84E-03 \\
\hline & $(1,3)$ & 0.045166 & 0.045196 & 0.045194 & A & $6.20 \mathrm{E}-02$ \\
\hline & $(2,2)$ & -0.080658 & -0.080367 & -0.080358 & B & $1.12 \mathrm{E}-02$ \\
\hline & $(2,3)$ & 0.095531 & 0.095401 & 0.095398 & A & $1.39 \mathrm{E}-01$ \\
\hline & $(3,3)$ & 0.728906 & 0.728187 & 0.728187 & B & $0.00 \mathrm{E}+00$ \\
\hline \multirow{6}{*}{$1 \mathrm{k}=23$} & $(1,1)$ & 0.590386 & 0.590318 & 0.590324 & $\mathrm{~A}$ & $1.05 \mathrm{E}-02$ \\
\hline & $(1,2)$ & -0.011834 & -0.011844 & -0.011846 & B & $1.69 \mathrm{E}-02$ \\
\hline & $(1,3)$ & 0.102070 & 0.102076 & 0.102076 & A & $5.88 \mathrm{E}-03$ \\
\hline & $(2,2)$ & 0.320864 & 0.320592 & 0.320592 & B & $0.00 \mathrm{E}+00$ \\
\hline & $(2,3)$ & 0.048173 & 0.048318 & 0.048319 & A & $3.03 \mathrm{E}-01$ \\
\hline & $(3,3)$ & 0.314492 & 0.315187 & 0.315196 & B & $2.86 \mathrm{E}-03$ \\
\hline \multirow{6}{*}{$1 \mathrm{k}=33$} & $(1,1)$ & 0.157583 & 0.157652 & 0.157647 & $\mathrm{~A}$ & $4.06 \mathrm{E}-02$ \\
\hline & $(1,2)$ & 0.314114 & 0.314087 & 0.314089 & B & $6.37 \mathrm{E}-04$ \\
\hline & $(1,3)$ & -0.045879 & -0.045924 & -0.045925 & A & $1.00 \mathrm{E}-01$ \\
\hline & $(2,2)$ & 0.822114 & 0.822369 & 0.822371 & B & $2.43 \mathrm{E}-04$ \\
\hline & $(2,3)$ & -0.034699 & -0.034885 & -0.034886 & A & 5.39E-01 \\
\hline & $(3,3)$ & -0.074520 & -0.075238 & -0.075247 & B & $1.20 \mathrm{E}-02$ \\
\hline
\end{tabular}




\subsubsection{Example B: BEM implementation - Isotropic material}

In this example, the proper implementation of the anisotropic fundamental solution based on Fourier series into a BEM code is tested. A short beam of length $2 L$ and square crosssection of side $H$, where $L=5 H$, is subjected to a uniformly distributed pressure load on its top surface, as shown in Figure 3.5 (a). Both ends of the beam are constrained in the three coordinate directions. Advantage is taken of symmetry and only half the beam was modelled in the BEM analysis, as shown in Fig. 3.5 (b). For the numerical analysis, the Young's modulus $E$ was taken to be 1000 and Poisson's ratio $\nu=0.3$.

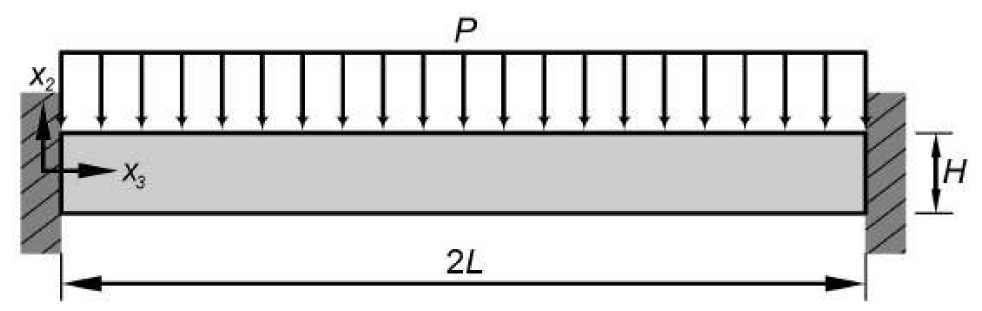

(a)

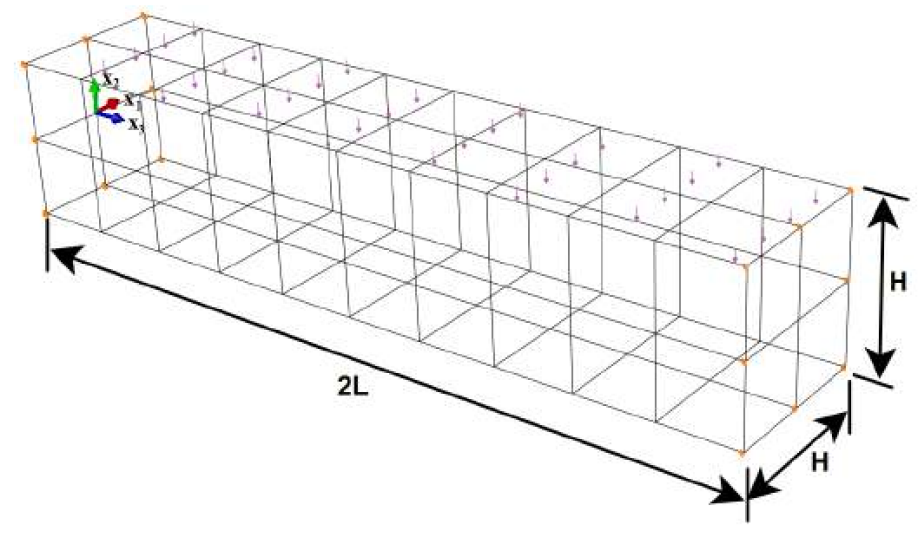

(b)

Figure 3.5: (a) Equivalent beam model; (b) BEM mesh (Symmetry).

A total of 88 quadratic quadrilateral continuous elements and 266 nodes are used to model the problem. For the Fourier series representation, $\alpha=16$ and 64 Gauss integration points were used. Normalized transverse displacements along the $x_{3}$-direction are computed and compared with the isotropic BEM formulation and simple beam theory, as shown in Fig. 3.6. 


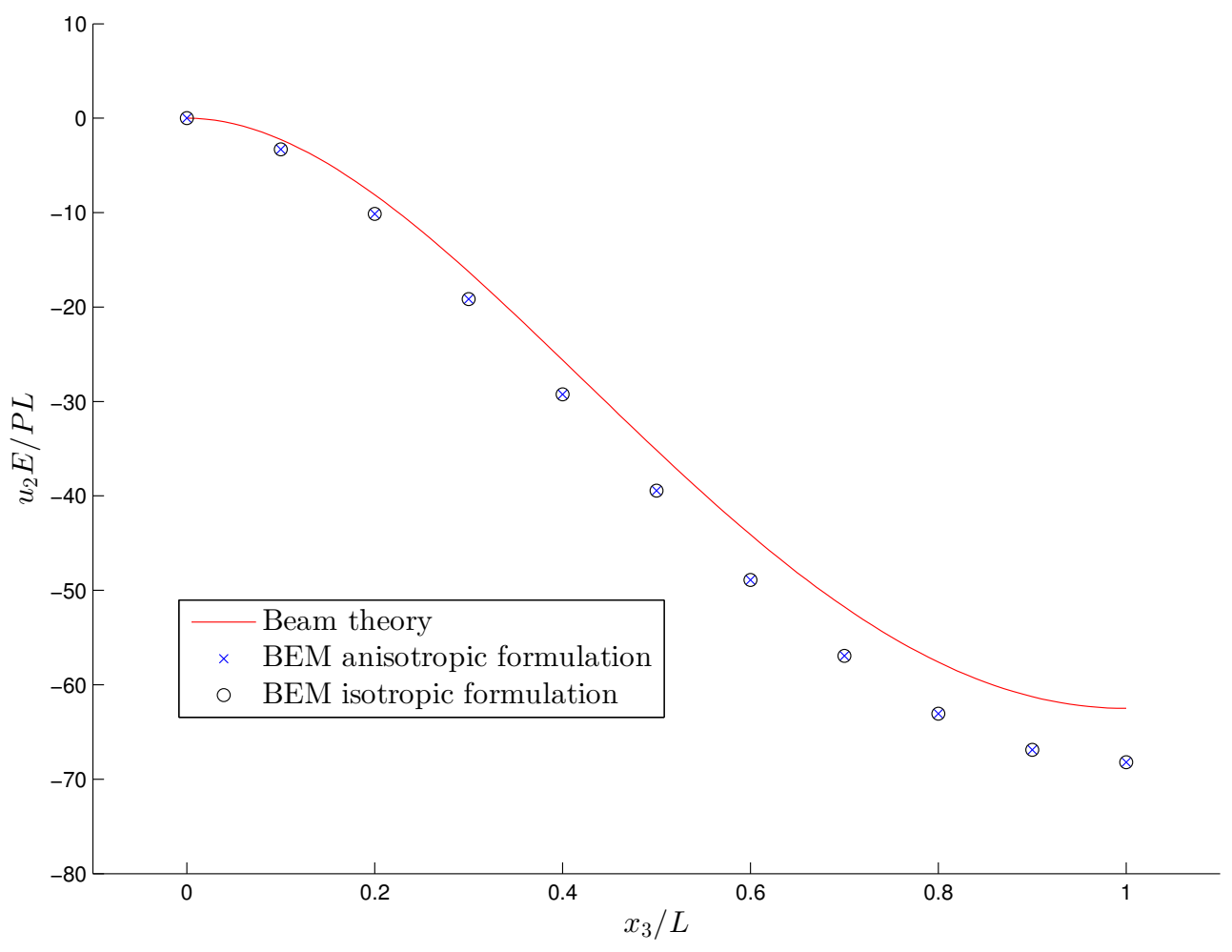

Figure 3.6: Normalized transverse displacements.

The normalized direct stress is also compared for cross-sections corresponding to $x_{3}=$ $2 H, 3 H, 4 H, 5 H$, as can be seen in Fig. 3.7. Even with the coarse BEM mesh, very good agreement of the results is observed when comparing the computed curves from the anisotropic and isotropic analyses. The maximum discrepancy between the numerical results and the analytical solution for simple beam theory is the normalized transverse displacement at $6.3 \%$ occurring at $x_{3}=L$. This discrepancy can be largely attributed to the non-zero Poisson's ratio used in the former and the fact that the beam is relatively short. 


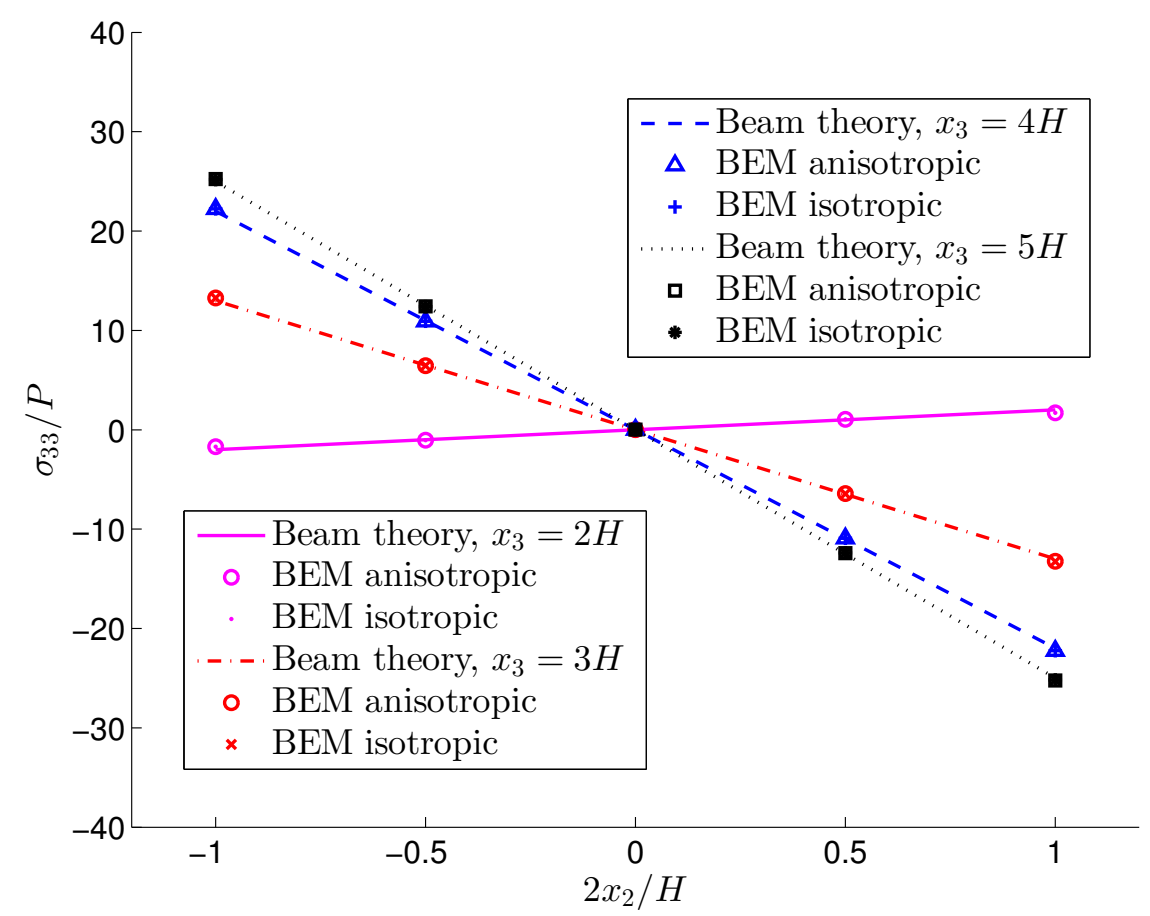

Figure 3.7: Normalized direct stresses at $x_{1}=2 H, 3 H, 4 H, 5 H$.

\subsubsection{Example C: BEM implementation - Alpha quartz crystal}

The physical problem in this example is a cube with a cylindrical hole. The radius of the hole is $r=0.3 H$, where $H$ is the length of its side, which are subjected to external pressure $P$, as shown in Fig. 3.8(a). The bottom and top surfaces are totally constrained in the three coordinate directions. The material considered is an alpha-quartz with the following stiffness matrix, (Huntington, 1958),

$$
\mathrm{C}=\left[\begin{array}{cccccc}
87.6 & 6.07 & 13.3 & 17.3 & 0 & 0 \\
6.07 & 87.6 & 13.3 & -17.3 & 0 & 0 \\
13.3 & 13.3 & 106.8 & 0 & 0 & 0 \\
17.3 & -17.3 & 0 & 57.2 & 0 & 0 \\
0 & 0 & 0 & 0 & 57.2 & 17.3 \\
0 & 0 & 0 & 0 & 17.3 & 40.765
\end{array}\right] \mathrm{GPa}
$$

A mesh of 1040 quadratic continuous quadrilateral elements was used, as shown in Fig. 
3.8 (b).
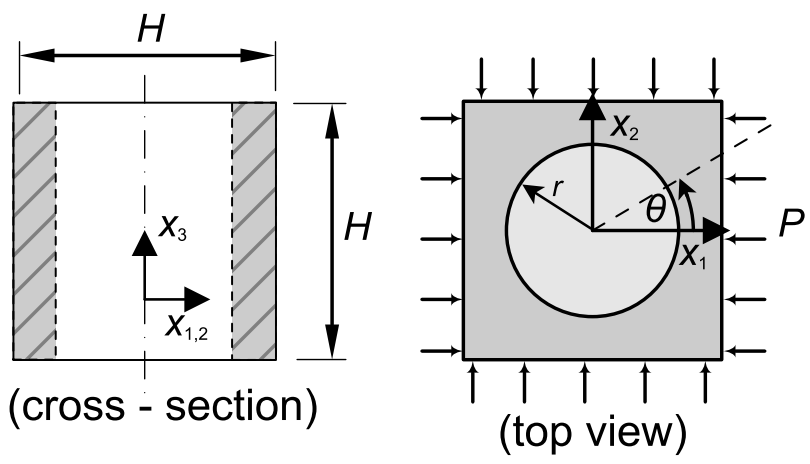

(a)

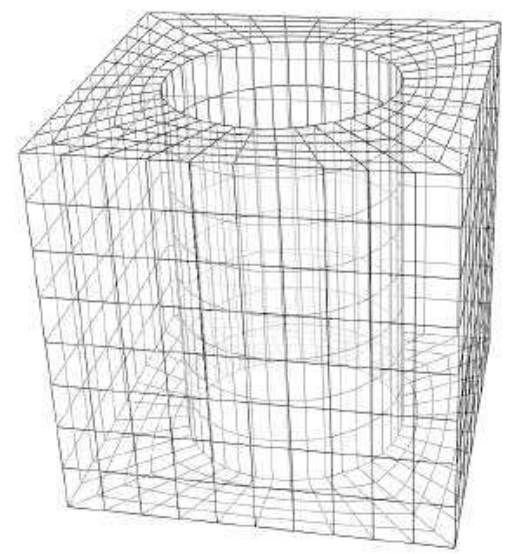

(b)

Figure 3.8: (a) geometry of Example C; (b) BEM mesh.

Results from the BEM anisotropic formulation are compared with the FEM results obtained by the commercial software Abaqus presented by Tan et al. (2013). The displacements in the $x_{1}$-direction on the internal cylindrical surface at $x_{3}=0.5 \mathrm{H}$ are compared along the circumferential position $\theta$. They are shown in Fig. 3.9, where it can be seen that there is good agreement between the two set of results. 


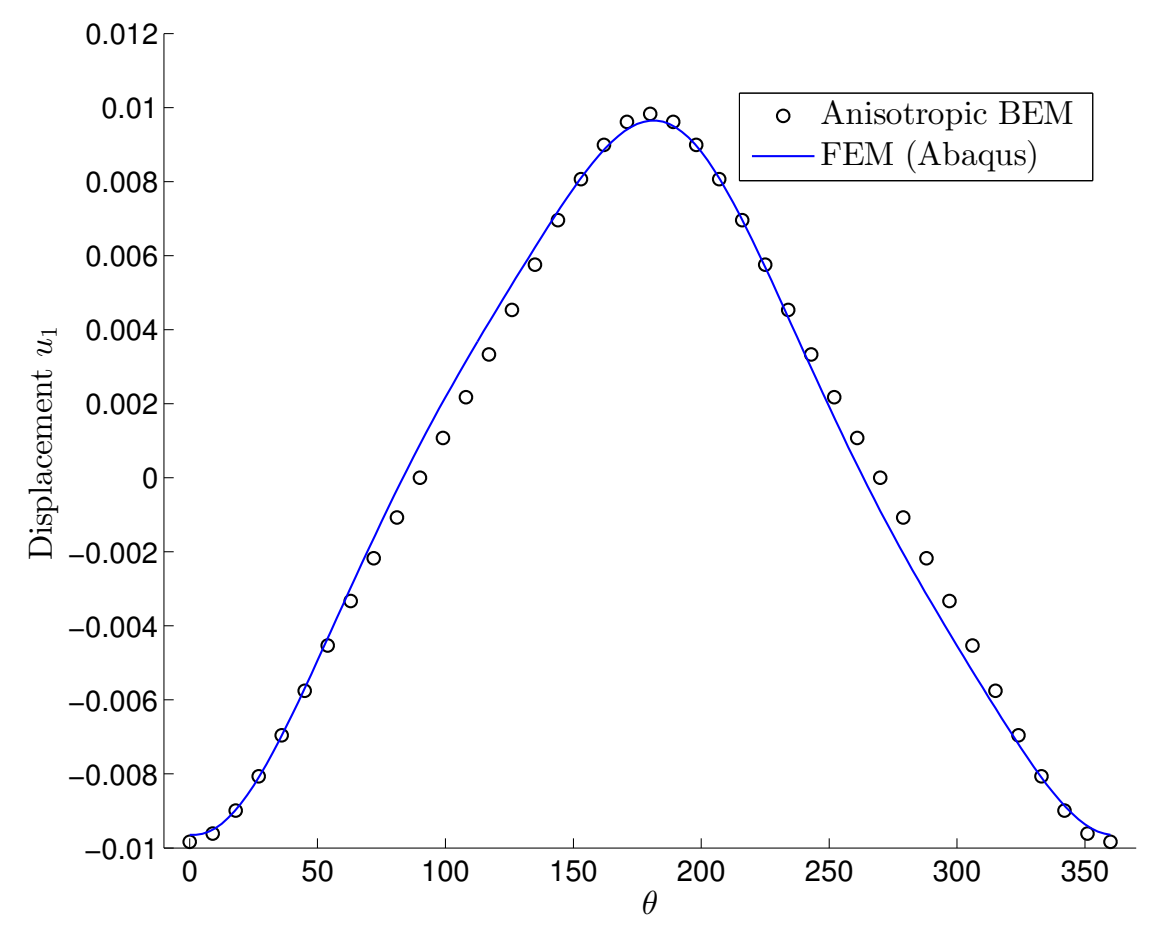

Figure 3.9: Comparison of the $u_{1}$ displacement around the circumferential hole in the mid-plane.

\subsubsection{Example D: BEM implementation - Generally anisotropic material}

The physical problem in this example is an internally pressurized cylinder made of an alpha-quartz crystal, as treated in (TAN et al., 2013). The main objective here is to verify the generally anisotropic fundamental solution when highly anisotropic materials are used. The problem considered is a cylinder subjected to internal pressure, $P$, with radius ratio $R_{2} / R_{1}=2$ and total length $2 H=8 R_{1}$, as shown in Figure 3.10(a). The external circumferential surface is constrained in the radial direction, while its two ends are fixed in the $x_{3}$ direction. The principal material axes of the alpha-quartz crystal are successively rotated about the global Cartesian $x_{1}$, $x_{2}$ and $x_{3}$ axis by $30^{\circ}, 45^{\circ}$ and $60^{\circ}$ clockwise, respectively. These successive rotations yield a fully populated stiffness matrix, as follows, 


$$
\mathbf{C}=\left[\begin{array}{cccccc}
111.8 & 14.8 & -5.2 & -0.3 & 11.0 & -14.0 \\
14.8 & 101.8 & -7.6 & 0.4 & -0.6 & 18.9 \\
-5.2 & -7.6 & 129.7 & 4.4 & 1.6 & 0.6 \\
-0.3 & 0.4 & 4.4 & 31.3 & 2.5 & 3.6 \\
11.0 & -0.6 & 1.6 & 2.5 & 37.9 & 1.3 \\
-14.0 & 18.9 & 0.6 & 3.6 & 1.3 & 55.2
\end{array}\right] \text { GPa }
$$

A mesh of 1232 quadratic continuous quadrilateral elements was analyzed, as shown in Fig. 3.10 (b).

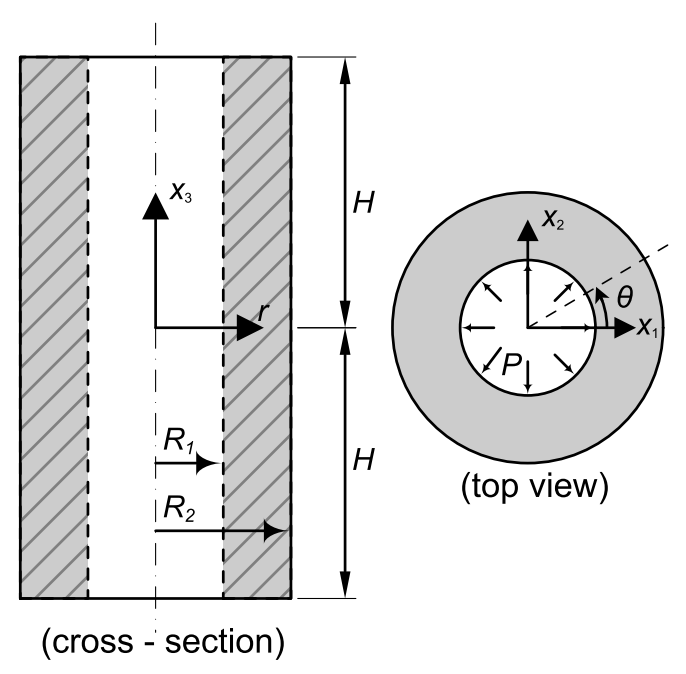

(a)

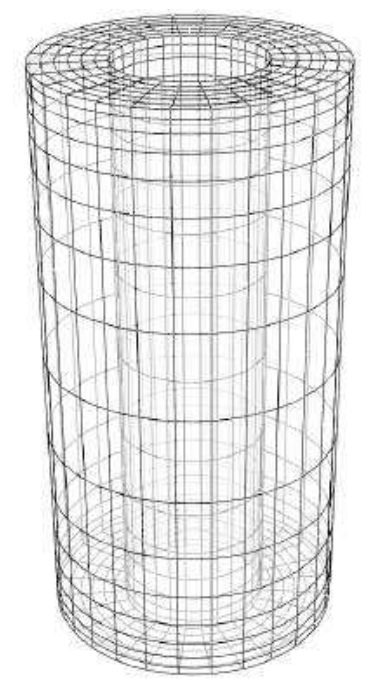

(b)

Figure 3.10: (a) geometry of example 3; (b) BEM mesh.

Results from the BEM anisotropic formulation are compared with the FEM results obtained by the commercial software ANSYS, carried out in (TAN et al., 2013). The normalized total displacement $\left(u_{T} C_{11} / P R_{1}\right)$ at $r=1.5 R_{1}$ on both ends is compared along the circumferential position $\theta$. The results are shown in Fig. 3.11, where again, excellent agreement of them is observed form the two different numerical methods. 


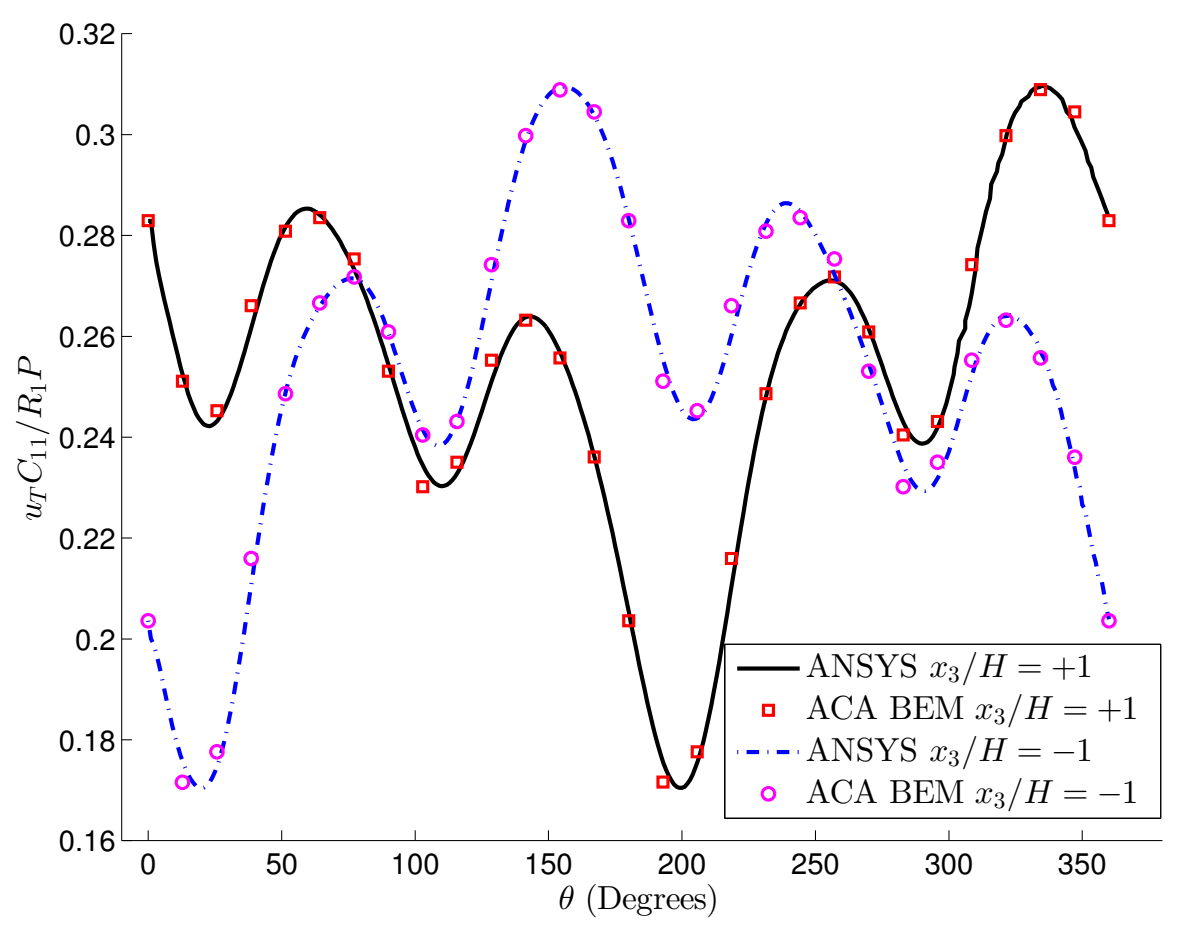

Figure 3.11: Normalized total displacement comparison.

\subsubsection{Example E: Multi-domain approach}

The correct implementation of the multi-domain approach applied to the 3D anisotropic formulation based on Fourier series is also tested. A beam of length $L$ and square cross-section of side $H$, where $L=12 H$, is subjected to a uniformly distributed pressure load on its top surface. One end of the beam is totally constrained in the three coordinate directions, while the other is constrained in the $x$ and $y$ direction, as shown in Fig. 3.12 (a). 


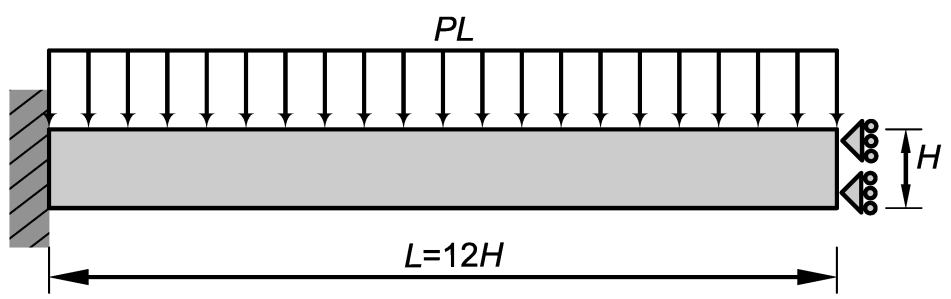

(a)

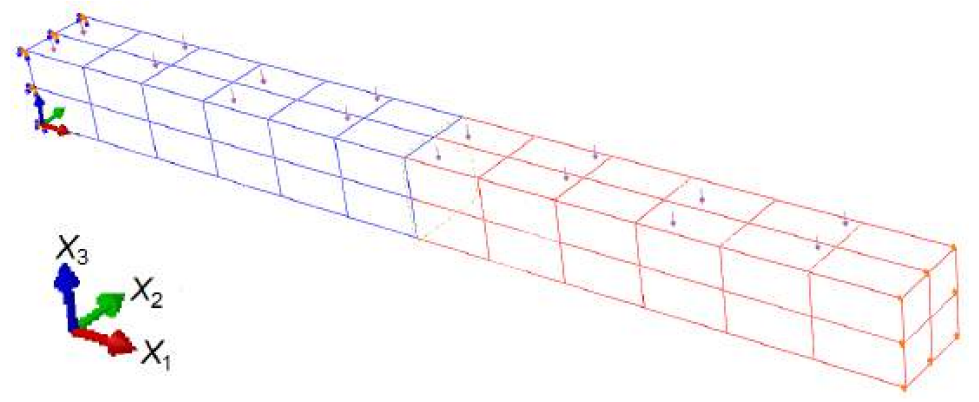

(b)

Figure 3.12: (a) Geometry and boundary conditions for example A; (b) BEM mesh.

A total of 112 quadratic quadrilateral elements and 319 nodes were used to model the problem. Two regions with the same anisotropic material were considered. The mesh and the region division can be seen in Fig. 3.12(b). The material considered is the same alpha-quartz crystal as in Example C.

Normalized transverse displacements along the $x_{1}$-direction are computed and compared with the simple region anisotropic BEM and the FEM method, as shown in Fig. (3.13). The normalized direct stress is also compared for cross-sections corresponding to $x_{1}=4 H, 6 H, 8 H$, as can be seen in Fig. 3.14. 


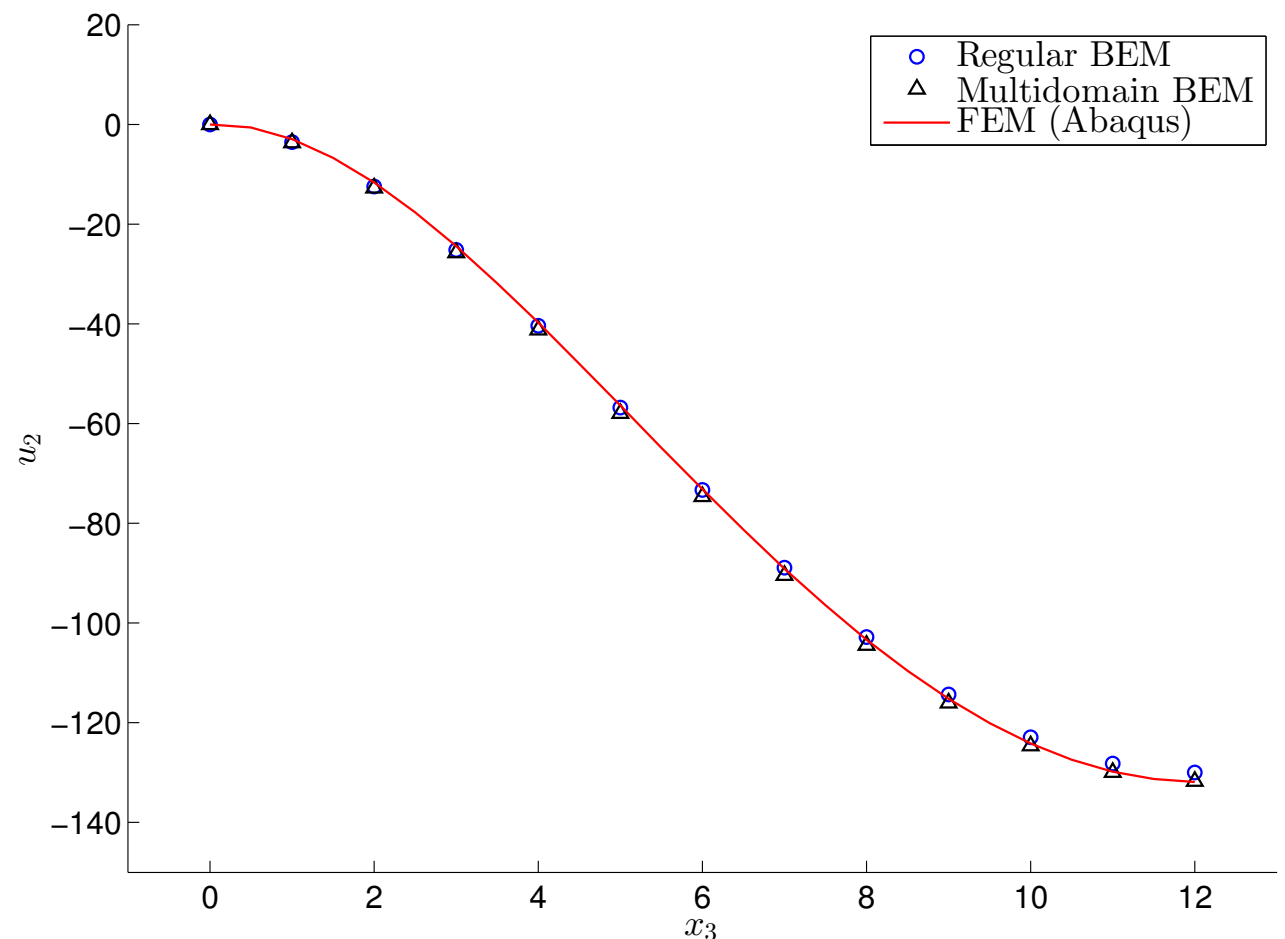

Figure 3.13: Normalized transverse displacements.

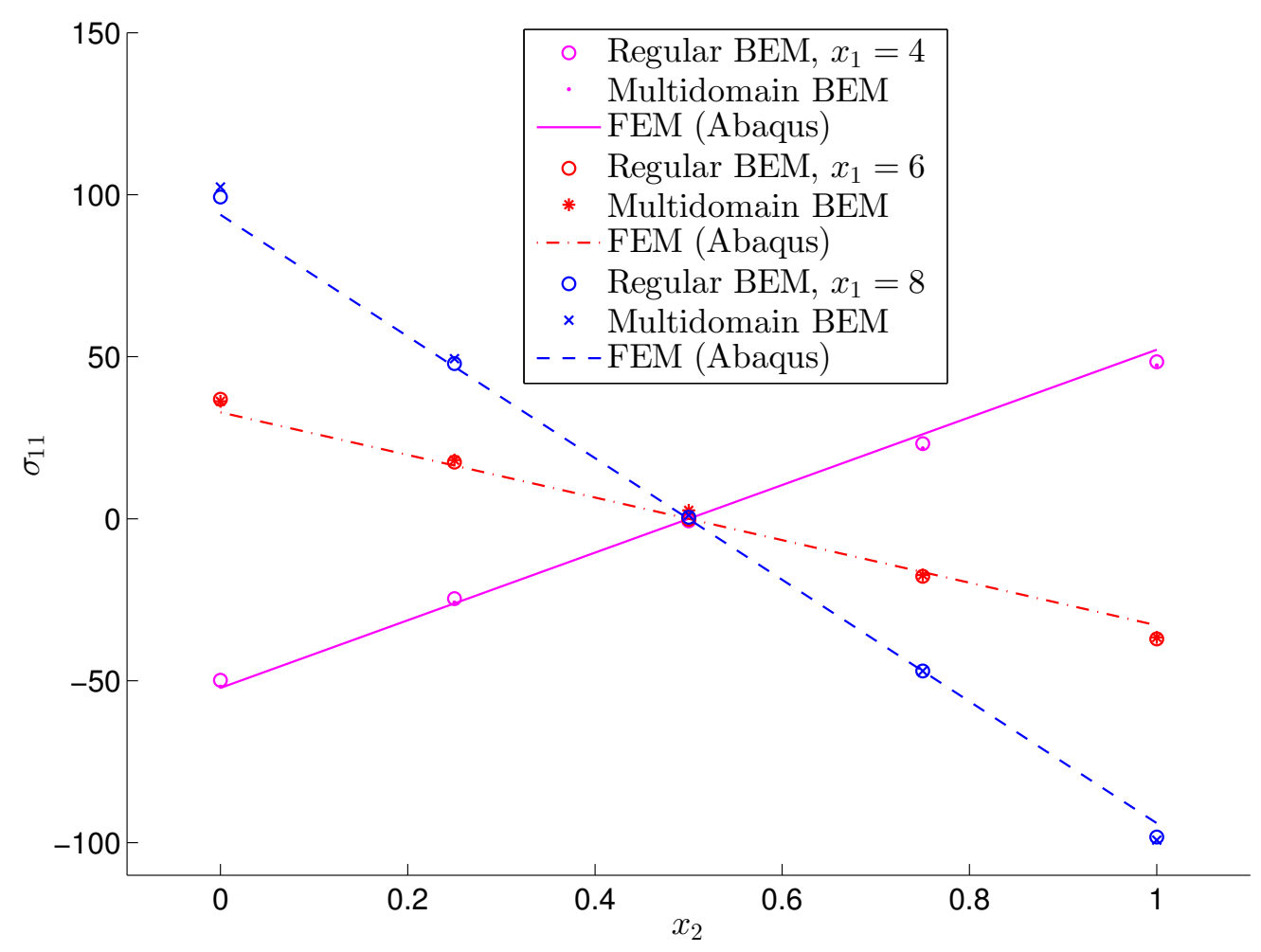

Figure 3.14: Normalized normal stresses at $x_{1}=4 H, 6 H, 8 H$. 
Good agreement is observed, despite the relatively coarse mesh used. The maximum discrepancy for displacements was $0.1 \%$ at $x_{1}=12 \mathrm{H}$, while the maximum discrepancy for the normal boundary stress is $5.4 \%$ at $x_{1}=8 H$ and $x_{2}=0$.

The same problem is considered but with different boundary conditions. Both ends of the beam are constrained in the three coordinate directions. The main objective here is to verify the generally anisotropic fundamental solution when it is used for different anisotropic materials within a multi-domain structure. An alpha-quartz crystal is considered for the first region, while, the principal material axes are rotated about the global Cartesian $x_{1}, x_{2}$ and $x_{3}$ axis by $30^{\circ}, 45^{0}$ and $60^{\circ}$ clockwise, respectively, for the second region. The successive rotations yield a fully populated stiffness matrix, as given in Equation (3.59).

The problem mesh is discretized with 832 quadratic quadrilateral elements. Results from the BEM anisotropic formulation are compared with the FEM results obtained by the commercial software Abaqus. The displacement $u_{3}$ is compared and results are shown in Fig. 3.15.

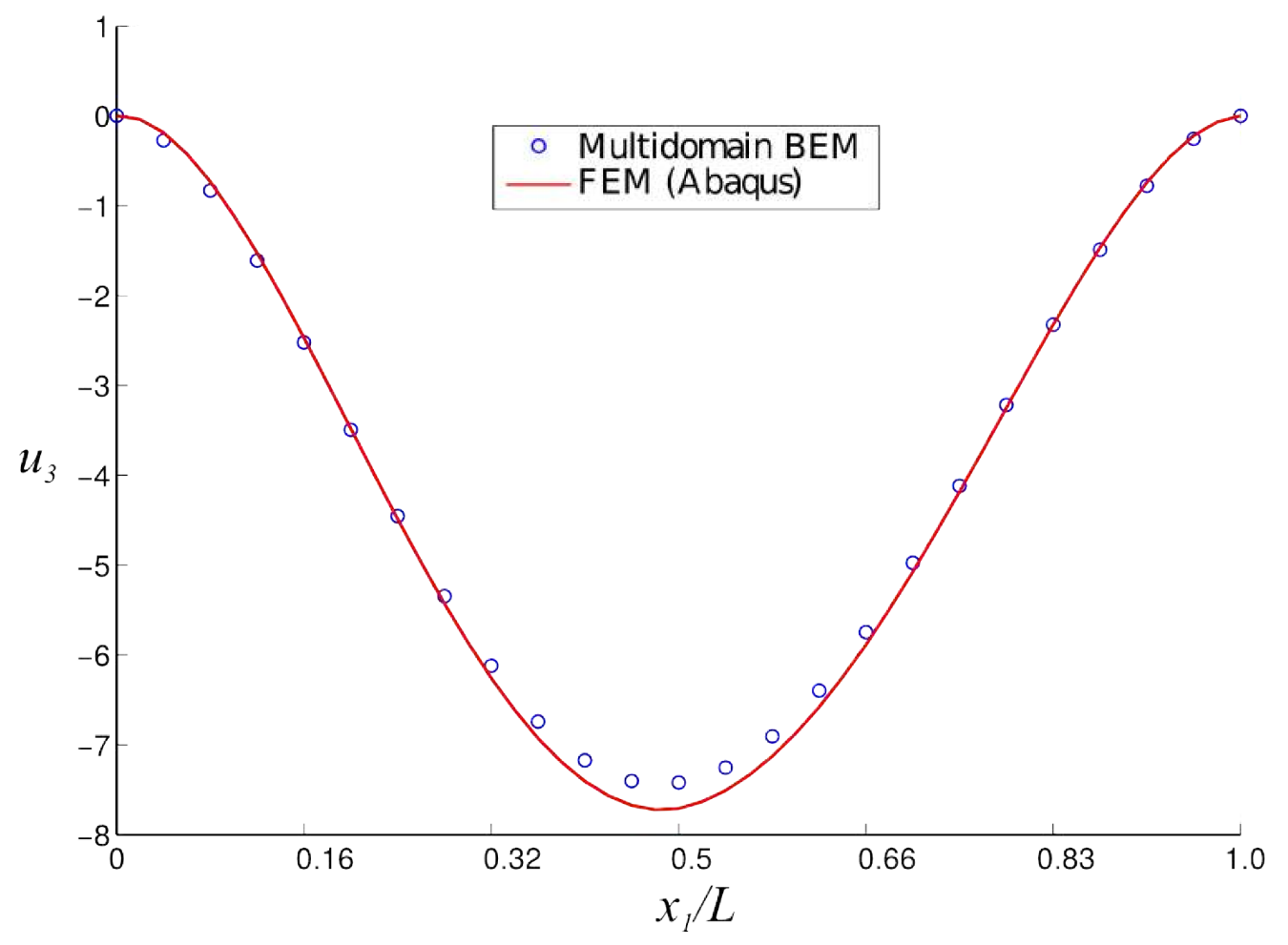

Figure 3.15: Normal displacement comparison. 


\section{The Boundary Element Method for 3D elastodynamics}

\subsection{Introduction}

In this chapter the BEM for 3D elastodynamics is studied. First, the integral elastodynamic representation, by means of the Boundary Integral Equation (BIE), is presented. Three methods are presented for the evaluation of the domain term in the elastodynamic BIE, namely the Dual Reciprocity Method (DRM), the Radial Integration Method (RIM) and the cell integration method. The direct analysis of dynamic problems and the Houbolt's algorithm for the time integration is then reviewed. Finally, numerical examples are presented to validate the implementation of the DRM and RIM for the transformation of the domain integrals in the BIE.

\subsection{Integral representation}

Two main approaches are known for the analysis of anisotropic materials in elastodynamics BEM problems. In the first approach, analytical fundamental solutions from elasto-statics are used and the inertial terms are considered as body forces. Domain integrals arising from inertial effects are transformed into boundary integrals. This approach will be used in the present work. In the second approach, numerical fundamental solutions are applied directly to elastodynamics. The use of these numerical fundamental solutions (Saez and Dominguez, 1999) demands a bigger computational effort and requires specific numerical treatments for good convergence.

The BIE for elastodynamics is obtained from the equilibrium relation for an elastodynamic state, applying the reciprocal theorem and integrating twice by parts. The BIE represents a reciprocal relation between an elastodynamic state and an elastostastic state, as follows,

$$
C_{k i}(\xi) u_{i}(\xi)+\int_{\Gamma} T_{k i} u_{i} \mathrm{~d} \Gamma=\int_{\Gamma} U_{k i} t_{i} \mathrm{~d} \Gamma-\int_{\Omega} \rho U_{k i}\left(b_{i}-\ddot{u}_{i}\right) \mathrm{d} \Omega
$$

where $C_{k i}(\xi)=\delta_{k i}$ when $\xi \in \Omega, C_{k i}(\xi)=0$ when $\xi \notin \Omega$, and $C_{k i}(\xi)=1 / 2 \delta_{k i}$ when $\xi \in \Gamma$ and the boundary is smooth at $\xi ; \rho\left(b_{i}-\ddot{u}_{i}\right)=p_{i}$ are the body forces (Dominguez, 1993). $U_{k i}$ and $T_{k i}$ are the elastostatics fundamental solutions for displacements and tractions, respectively. The main difficulty arises from evaluating the domain integral of Equation (4.1). 
The application of the BEM, in most cases, requires the fundamental solution for the problem to be previously known. This solution should consider all terms in the governing equation in order to obtain a formulation in which just the boundary is discretized. When this is not possible, the terms that were not consider in the fundamental solution will produce domain integrals, as that obtained in Equation (4.1). The domain integrals can also be evaluated or transformed into boundary ones. Otherwise, the boundary element formulation would lose its main characteristic which is the boundary only-discretization. The evaluation of the domain terms can also be accomplished by cell integration. The first alternative is to accomplish an exact transformation of the domain integrals into boundary integrals when the body forces are known, as shown by Venturini (1988) for isotropic plate bending problems, by Gao (2002) for anisotropic three-dimensional problems, and by Albuquerque et al. (2006) for anisotropic plate bending problems. The second alternative is to transfer the effects of the domain integrals into the boundary using approximation methods, such as the DRM or RIM. These methods are more general and could be applied to body forces that depend on the solution of the problems, such as those encountered in dynamic transient problems in which inertial effects are considered. The DRM has been successfully used in the dynamic analysis of anisotropic structures as presented by (Albuquerque et al., 2002), (Albuquerque et al., 2003a), (Albuquerque et al., 2003b) and (Albuquerque et al., 2004) for bi-dimensional problems, and by (Kögl and Gaul, 2000a), (Kögl and Gaul, 2000b) and (Kögl and Gaul, 2003) for three-dimensional problems. Due to the complexity of the governing equations of anisotropic materials, the analytical evaluation of particular solutions used in the DRM is restricted to some approximation functions, all of them are function of material properties, (ALBUQUERQUE et al., 2007b). In general, there are several methods in literature for the domain integral evaluation, such as, analytical integration of the domain integrals, the use of Fourier expansions, the Galerkin Vector Technique, the Multiple Reciprocity Method, high-order fundamental solutions, the cell integration method, the Dual Reciprocity Method (DRM), (PARTRIDGE et al., 1992), the Radial Integration Method (RIM), (GAO, 2002), among others. The three latter methods are the most popular among domain integral transformation methods. According to Gaul et al. (2003), among these methods, the DRM is the only general technique that can be used for all types of the domain term.

\subsection{Cell integration method}

In the cell integration method the domain $\Omega$ is subdivided into internal cells $\Omega_{k}$ in which the integral is accomplished, as shown in Figure 4.1. The cells have the appearance of a FEM mesh. When the body forces are known, the procedure has, however, an essential difference because there are no unknowns in the domain and the cells are only used to carry out the 
analytical integration or Gaussian quadrature, (GAUL et al., 2003).

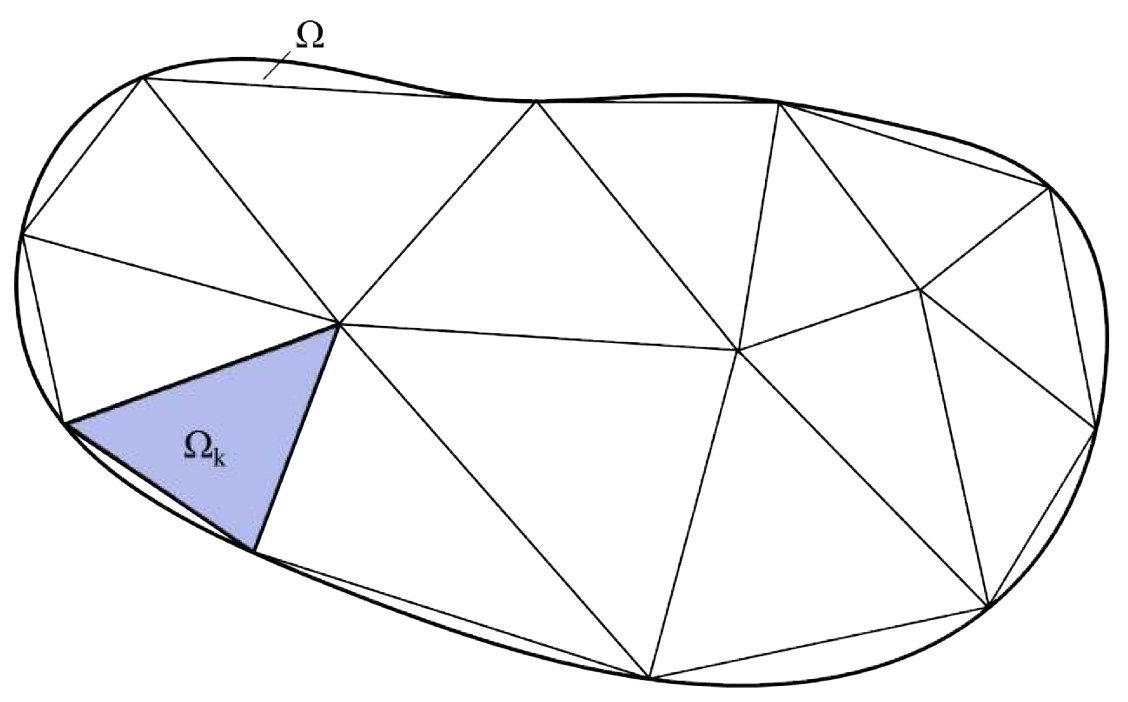

Figure 4.1: Cell domain discretization with integration cells $\Omega_{k}$ in a $2 \mathrm{D}$ problem.

In a general form, the cell integration of the domain integral of Equation (4.1) can be expressed as, (USECHE, 2007),

$$
\int_{\Omega} U_{k i} p_{i} \mathrm{~d} \Omega=\sum_{k=1}^{n c e l l s} \int_{\Omega_{k}} U_{k i} p_{i} \mathrm{~d} \Omega_{k}
$$

Generally, the evaluation of the discretized domain integrals can be obtained by numerical integration, e.g., using Gaussian quadrature in each cell. The domain integral in Equation (4.2) can be rewritten as, (Partridge et al., 1992),

$$
d_{i}=\int_{\Omega} U_{k i} p_{i} \mathrm{~d} \Omega=\sum_{k=1}^{n c e l l s}\left[\sum_{e=1}^{n p g} \omega_{e}\left(U_{k i} p_{i}\right)_{e}\right] \Omega_{k}
$$

where $e$ is the Gaussian integration point, $n p g$ is the total number of integration points, $\omega_{e}$ is the Gaussian icntegration weights and $\Omega_{k}$ is the area of the $k$ cell. $\left(U_{k i} p_{i}\right)$ needs to be evaluated at integration points $e$ on each cell. Further information on the cell integration method is available in (Kane, 1994). 
Recently, Carrer et al. (2013) applied the cell integration method to dynamic analysis of Timoshenko isotropic beams. Useche and Harnish (2016) also applied the cell integration method for modal analysis of doubly curved thick shallow shells. To the best of author's knowledge, there is no work in literature where the domain integrals that come from inertia effects are treated by cells for anisotropic materials. Although possible, it loses the main characteristics of a BEM formulation that is the discretization of boundary only.

\subsection{Dual Reciprocity Formulation (DRM)}

The BIE presented in Equation (4.1) is not a pure boundary representation of the displacement field $u_{i}$, because of the existence of domain integrals involving body forces and the inertia term. By the use of the DRM, it is possible to transform domain integrals into boundary integrals. The DRM was initially proposed by Nardini and Brebbia (NARDINI AND BREBBIA, 1982) and applied to elastodynamic problems. Wrobel and Brebbia (WROBEL AND BREBBIA, 1987) extended the DRM to time-dependent difussion problems. A complete bibliography of the DRM applications can be found in (PARTRIDGE et al., 1992).

In the DRM, the term $p_{i}$ is approximated as a sum of $\mathrm{N}$ tensor functions $f_{m k}^{j}$ and unknown functions $\alpha_{k}^{j}$, as follows,

$$
p_{i}=\rho\left(b_{i}-\ddot{u}_{i}\right) \approx \sum_{j=1}^{N} f_{m k}^{j} \alpha_{k}^{j}
$$

where $f_{m k}^{j}$ have to fulfil the equilibrium equation, in the D'Alembert sense,

$$
C_{m n r s} \hat{u}_{r k, n s}^{j}=f_{m k}^{j}
$$

The solutions of Equation (4.5), $\hat{u}_{r k}$, are known as particular solutions. The reciprocal integral relation can also be obtained between the fundamental solution and the particular solution, as follows,

$$
C_{l i} \hat{u}_{i n}^{m}+\int_{\Gamma} T_{l i} \hat{u}_{i n}^{m} \mathrm{~d} \Gamma=\int_{\Gamma} U_{l i} \hat{t}_{i n}^{m} \mathrm{~d} \Gamma-\int_{\Omega} f^{m} U_{l n} \mathrm{~d} \Omega
$$


A new representation for the displacement field in terms of particular solutions can be obtained by substituting Equation (4.6) and Equation (4.4) into Equation (4.1),

$$
C_{l i} u_{i}+\int_{\Gamma} T_{l i} u_{i} \mathrm{~d} \Gamma=\int_{\Gamma} U_{l i} t_{i} \mathrm{~d} \Gamma+\rho \sum_{m=1}^{M} \alpha_{n}^{m}\left\{C_{l i} \hat{u}_{i n}^{m}-\int_{\Gamma} U_{l i} \hat{t}_{i n}^{m} \mathrm{~d} \Gamma+\int_{\Gamma} T_{l i} \hat{u}_{i n}^{m} \mathrm{~d} \Gamma\right\}
$$

Equation (4.7) is the basis of DRM, in which, it can be observed the absence of domain integrals.

There are several methods for the calculation of particular solutions, such as, (AtKinson, 1985), (Grundemann, 1989), (GolberG, 1995), (KöGl And Gaul, 2000a). Most of them consider radial functions for the tensor functions $f_{m k}^{j}$ and solve the differential equation presented in Equation (4.1). Schclar (SCHCLAR, 1994) proposed a simpler approach, in which a radial function is chosen for $\hat{u}_{r k}$ and the tensor functions are obtained by derivation from Equation (4.5). This approach is also used in the present project. The particular solution, as recommended for anisotropic materials by (KÖGL AND GAUL, 2000a), is chosen as,

$$
\hat{u}_{r k}=\delta_{k n}\left(r^{2}+r^{3}\right)
$$

which yields the derivatives

$$
\begin{gathered}
\hat{u}_{r k, l}=\delta_{r k}\left(2 r+3 r^{2}\right) r_{, l}, \\
\hat{u}_{r k, l j}=\delta_{r k}\left((2+3 r) \delta_{l j}+3 r r_{, j} r_{, l}\right)
\end{gathered}
$$

\subsection{The Radial Integration Method (RIM)}

An alternative approach for transforming domain integrals into boundary integrals based on radial integration was proposed by Gao (GAO, 2001). This approach, as stated by (GAO, 2002), is based on pure mathematical treatments, so it could be applied to transform any domain integral without the necessity of using particular solutions. For the case of domain integrals consisting of known functions the transformation is straightforward and accurate, while 
for domain integrals in which unknown functions are involved the transformation is accomplished with the use of Radial Basis Functions (RBF) to approximate the unknown quantities as in the DRM. Transformations are simple and follow the same procedure in 2D and 3D problems, making the method more attractive and easier to implement.

Taking a general 3D domain $\Omega$ bounded by $\Gamma$, as shown in Figure (4.2).

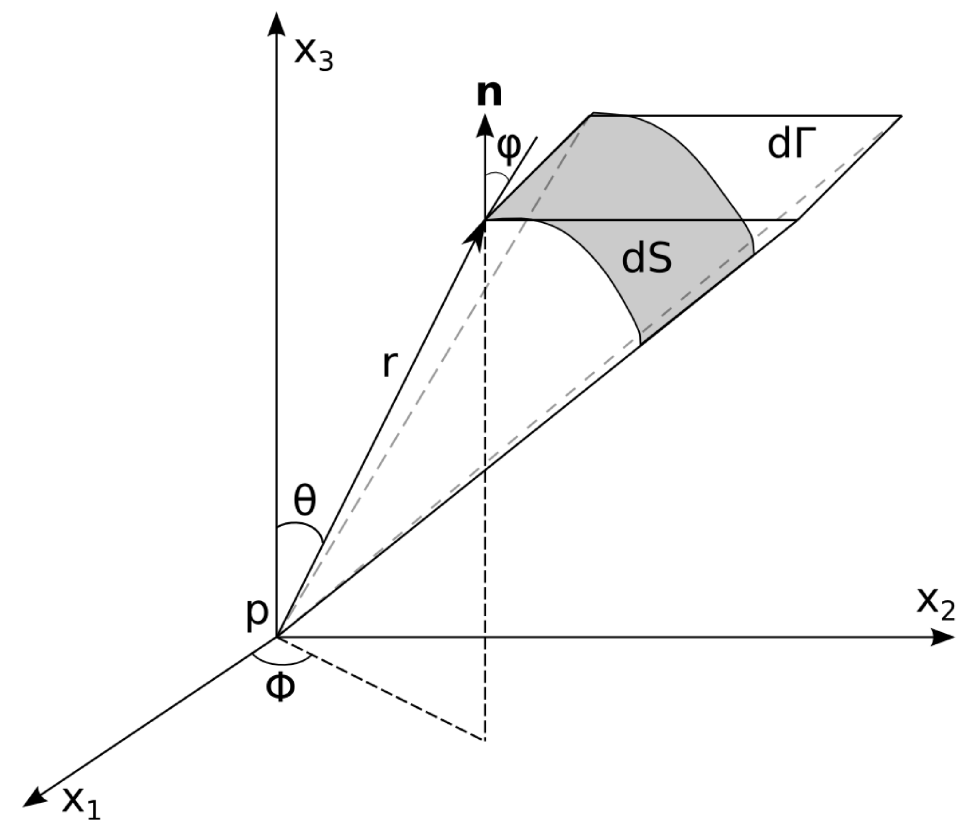

Figure 4.2: General 3D domain differential.

The Cartesian and the spherical coordinate systems are related as follows,

$$
\begin{aligned}
& r_{1}=x_{1}-x_{1}^{p}=r \sin \theta \cos \phi \\
& r_{2}=x_{2}-x_{2}^{p}=r \sin \theta \sin \phi,(0 \leq \theta \leq \pi, 0 \leq \phi \leq 2 \pi) \\
& r_{3}=x_{3}-x_{3}^{p}=r \cos \theta
\end{aligned}
$$

The origin is set at the source point $p . x_{i}^{p}$ represents the Cartesian coordinates at the source point, while $r$ represents the distance between the source and the field point $x_{i}$.

A differential domain $\mathrm{d} \Omega$ in spherical coordinates can be expressed as $\mathrm{d} \Omega=$ $r^{2} \sin \theta \mathrm{d} r \mathrm{~d} \theta \mathrm{d} \phi$. Then, for a general function $f(x)$, its domain integral can be written as 


$$
\int_{\Omega} f(x) \mathrm{d} \Omega=\int_{0}^{2 \pi} \int_{0}^{\pi} \int_{0}^{r(\theta, \phi)} f(r, \theta, \phi) r^{2} \mathrm{~d} r \sin \theta \mathrm{d} \theta \mathrm{d} \phi
$$

A function $F(\theta, \phi)$ can be defined as

$$
F(\theta, \phi)=\int_{0}^{r(\theta, \phi)} f(r, \theta, \phi) r^{2} \mathrm{~d} r
$$

The integral of Equation (4.11) can then be expressed as

$$
\int_{\Omega} f(x) \mathrm{d} \Omega=\int_{0}^{2 \pi} \int_{0}^{\pi} F(\theta, \phi) \sin \theta \mathrm{d} \theta \mathrm{d} \phi
$$

The differential area $\mathrm{d} S$ can be expressed in spherical coordinates by $\mathrm{d} S=r^{2} \sin \theta \mathrm{d} \theta \mathrm{d} \phi$. Also, from Figure (4.2), the relationship between the differential spherical surface $(\mathrm{d} S)$ and the differential real boundary surface $(\mathrm{d} \Gamma)$ can be written as

$$
\mathrm{d} S=\mathrm{d} \Omega \cos \varphi=\mathrm{d} \Gamma \frac{r_{i} n_{i}}{r(Q)}
$$

where $\varphi$ is the angle between $\mathbf{r}$ and the boundary surface $\mathrm{d} \Gamma$ outward normal $\mathbf{n}$, as can be seen in Figure (4.2). $Q$ indicates that the field point is on the boundary $\Gamma$. Substituting Equation (4.14) and Equation (4.12) into Equation (4.11) leads to

$$
\sin \theta \mathrm{d} \theta \mathrm{d} \phi=\frac{r_{i} n_{i}}{r^{3}} \mathrm{~d} \Gamma=\frac{1}{r^{2}(Q)} \frac{\partial r}{\partial n} \mathrm{~d} \Gamma
$$

where $\partial r / \partial n=r_{, i} n_{i}$ and $r_{i}=\partial r / \partial x_{i}=r_{i} / r$.

Finally, substituting Equation (4.15) into Equation (4.11) leads to

$$
\int_{\Omega} f(x) \mathrm{d} \Omega=\int_{\Gamma} \frac{1}{r^{\alpha}(Q)} \frac{\partial r}{\partial n} F(Q) \mathrm{d} \Gamma(Q)
$$


where

$$
F(Q)=\int_{0}^{r(Q)} f(x) r^{\alpha} \mathrm{d} r
$$

where $\alpha=1$ for 2D problems and $\alpha=2$ for 3D problems. The expression of Equation (4.16) is valid for the source point located at internal or boundary nodes. Moreover, weak singularities involved in the integrant $f(x)$ have been transformed to the boundary, as a result, no singularities exist at internal nodes for such integrants (GAO, 2002).

Similar to the DRM Section, the domain integral to be integrated is

$$
I(Q)=\int_{\Omega} U_{k i} p_{i} \mathrm{~d} \Omega
$$

In the RIM, the term $p_{i}$ is approximated as

$$
p_{i}=\rho\left(b_{i}-\ddot{u}_{i}\right) \approx \sum_{m=1}^{M} \gamma^{m} f^{m}
$$

Substituting Equation (4.19) into Equation (4.18) and transforming the differential domain $\mathrm{d} \Omega$ to spherical coordinates, we have

$$
I(Q)=\sum_{m=1}^{M} \gamma^{m} \int_{\Omega} f^{m} U_{k i}(Q, P) r^{2} \mathrm{~d} r \sin \theta \mathrm{d} \theta \mathrm{d} \phi
$$

Applying the relationship of Equation (4.15) into Equation (4.20) leads to

$$
I(Q)=\sum_{m=1}^{M} \gamma^{m} \int_{\Gamma} F^{m}(Q, P) \frac{\partial r}{\partial n} \frac{1}{r^{2}} \mathrm{~d} \Gamma
$$

where 


$$
F^{m}(Q, P)=\int_{0}^{r} f^{m} U_{k i}(Q, P) r^{2} \mathrm{~d} r
$$

\subsection{Dynamic analysis: transient analysis}

The solution of transient problems can be accounted by a large number of timestepping schemes, as presented in (WOOD, 1990). For elastodynamics, the Houbolt's algorithm, (HoubOLT, 1950), has demonstrated to yield better results when compared with other methods such as the Newmark algorithm, (NEWMARK, 1959), the central difference scheme, the Park's algorithm, (PARK, 1975), among others. This fact was demonstrated by Loeffler and Mansur (1987) for 2D problems and by Agnantiaris et al. (1996) for 3D problems and was attributed to the inherent numerical damping in the Houbolt's algorithm, which affects higher modes and hence minimize the problem resulting from dismissing complex eigenfrequencies, (GAUL et al., 2003).

There are two main approaches to deal with time integration when solving the equation of motion. The direct methods and the modal superposition approach. The direct methods employ an appropriate step by step procedure in which the equations of motion are satisfied at discrete time steps. In the modal superposition approach the equation of motion is transformed into a modal space in order to decouple the equations. Then, the step by step time integration is performed on the decoupled equations, and the solution is transformed back into the physical space. In the present work, just the direct analysis will be explored. For further information on the latter approach, please refer to (GAUL et al., 2003).

\subsubsection{Direct analysis and the Houbolt's algorithm}

In the direct analysis the total time period $T$ is divided into $N$ equal time steps $\Delta t$. Assuming that the solution at $t=0$ is known, it is possible to evaluate the solution at time step $n=1$ and subsequently the solution at time steps $n=2, n=3$, and so on. Hence, the solution at time step $n+1$ is obtained from the preceding time step $n$ or earlier time steps $n-1, n-2$, etc. As the equation of motion is a system of ordinary differential equations with constant coefficients, it can be solved using a variety of finite difference schemes. If the equation is solved at time step $n$, the time stepping scheme is called explicit, while, if it is solved at time step $n+1$, the time stepping scheme is called implicit. The latter scheme is usually attributed better 
stability properties, and, hence, it was implemented in the present work.

The resulting discretized equation can be written in matrix form for a time step $n+1$ as,

$$
\rho \mathbf{M} \ddot{\mathbf{u}}_{n+1}+\mathbf{H} \mathbf{u}_{n+1}=\mathbf{G} \mathbf{t}_{n+1}
$$

For its stability, the implicit integration scheme of Houbolt's was implemented. The Houbolt's algorithm is a backward-type finite difference scheme in which the velocity and the acceleration are approximated by displacement values at preceding time steps, as follows,

$$
\begin{gathered}
\dot{u}_{n+1} \approx \frac{1}{6 \Delta t}\left(11 u_{n+1}-18 u_{n}+9 u_{n-1}-2 u_{n-2}\right), \\
\ddot{u}_{n+1} \approx \frac{1}{\Delta t^{2}}\left(2 u_{n+1}-5 u_{n}+4 u_{n-1}-u_{n-2}\right)
\end{gathered}
$$

Substituting Equation (4.25) into Equation (4.23),

$$
\left[\frac{2}{\Delta t^{2}} \rho \mathbf{M}+\mathbf{H}\right] \mathbf{u}_{n+1}=\mathbf{G} \mathbf{t}_{n+1}+\frac{1}{\Delta t^{2}} \rho \mathbf{M}\left(5 \mathbf{u}_{n}-4 \mathbf{u}_{n-1}+\mathbf{u}_{n-2}\right)
$$

Displacements $\mathbf{u}_{n}, \mathbf{u}_{n-1}$ and $\mathbf{u}_{n-2}$ are known and, thus, the system of equations in Equation (4.26) can be solved using the boundary conditions at time step $(n+1)$. A new system of equations can be created by leaving all the set of unknown variables at the left hand side,

$$
\mathrm{Ax}_{n+1}=\mathbf{f}_{n+1}
$$

where $\mathbf{x}_{n+1}$ is the vector of unknown variables and $\mathbf{f}_{n+1}$ is the vector of known variables. The matrix $\mathbf{A}$ and the vector $\mathbf{f}_{n+1}$ are obtained from displacement values at previous steps and boundary conditions at step $n+1$. Once the system of Equation (4.27) is solved, $\mathbf{u}_{n+1}$ is known, and the solution can be obtained for the next time step. 


\subsection{Numerical examples}

Four numerical examples are presented in this section. In the first example, the DRM and the RIM are tested for the domain integral transformation when a known body force is considered for static problems. The known body force is a gravitational load $(0,0,-\gamma g)$. In the example $\mathrm{B}$, the multi-domain approach is tested for anisotropic materials when subjected to gravity load. In the example $\mathrm{C}$, the transient analysis for a step load is studied on an isotropic bar. Both methods, the DRM and the RIM, are explored. Finally, in example D, the same geometry and material properties are used, but with a transient ramp load. The DRM was used to this end. In all examples results were compared to analytical solutions and good agreement was observed in all cases. The time step $\Delta t$ and the mesh refinement are critical for the convergence of results. Some conclusions on the influence of these parameters are also presented.

\subsubsection{Example A: Simple body force - gravity}

In this example the static implementation of the domain integral transformation for a simple and known body force is tested. Both the results from the DRM and the RIM are compared to the analytical solution. The known body force is a gravitational load $(0,0,-\gamma g)$; where $g$ is the acceleration due to gravity and $\gamma$ is the specific weight of the material. The bar of length $2 u$ and square cross-section of side $1 u$ is subjected to its own weight, as shown in Figure 4.3. The top end of the bar is constrained in the $Z$ direction, while the bottom end of the bar is constrained in the $X$ and $Y$ directions. 


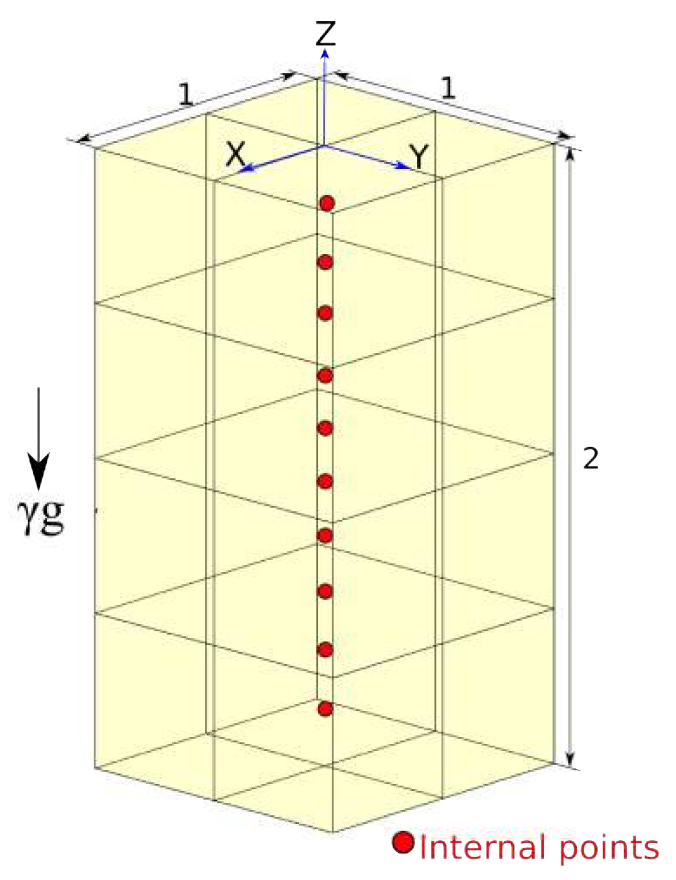

Figure 4.3: Bar stretched by its own weight.

The bar was modelled with two elements in the $X$ and $Y$ coordinate directions and four elements in the $Z$ coordinate direction. A total of 40 quadratic discontinuous elements and 320 physical nodes were created to model the problem. Moreover, 10 internal nodes were equally distributed generated at the $Z$ axis $(0,0,-Z)$, as can be also seen in Figure 4.3. The elastic properties for the problem are $E=10000, \nu=0.3$ and $\gamma=1$. The displacements in the $z$ direction, $u_{3}$, at internal nodes are compared to results obtained from the well-known analytical solution, (Timoshenko and Goodier, 1970)

$$
u_{3}=\frac{\gamma g(L-Z)^{2}}{2 E}+\frac{\nu \gamma g}{2 E}\left(X^{2}+Y^{2}\right)-\frac{\gamma g L^{2}}{2 E}
$$

where $L$ is the length of the bar. Results for the displacements $u_{3}$, are shown in Figure 4.4. Those obtained from DRM and RIM, as well as the error when compared with the analytical solution, are listed in Table 4.1. The same values are shown graphically in Figure 4.5. 


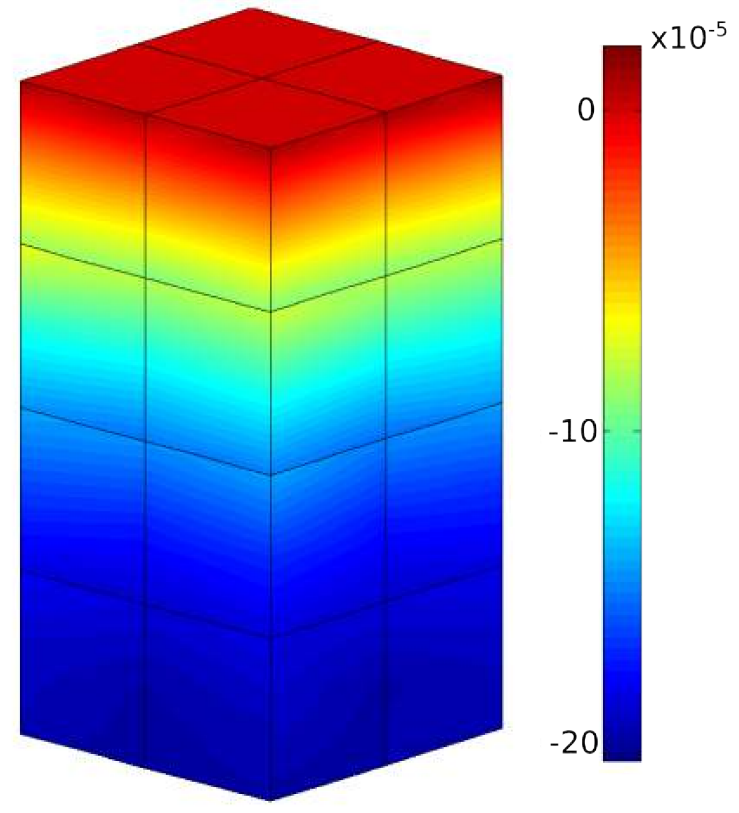

Figure 4.4: $u_{3}$ displacements for a bar stretched by its own weight.

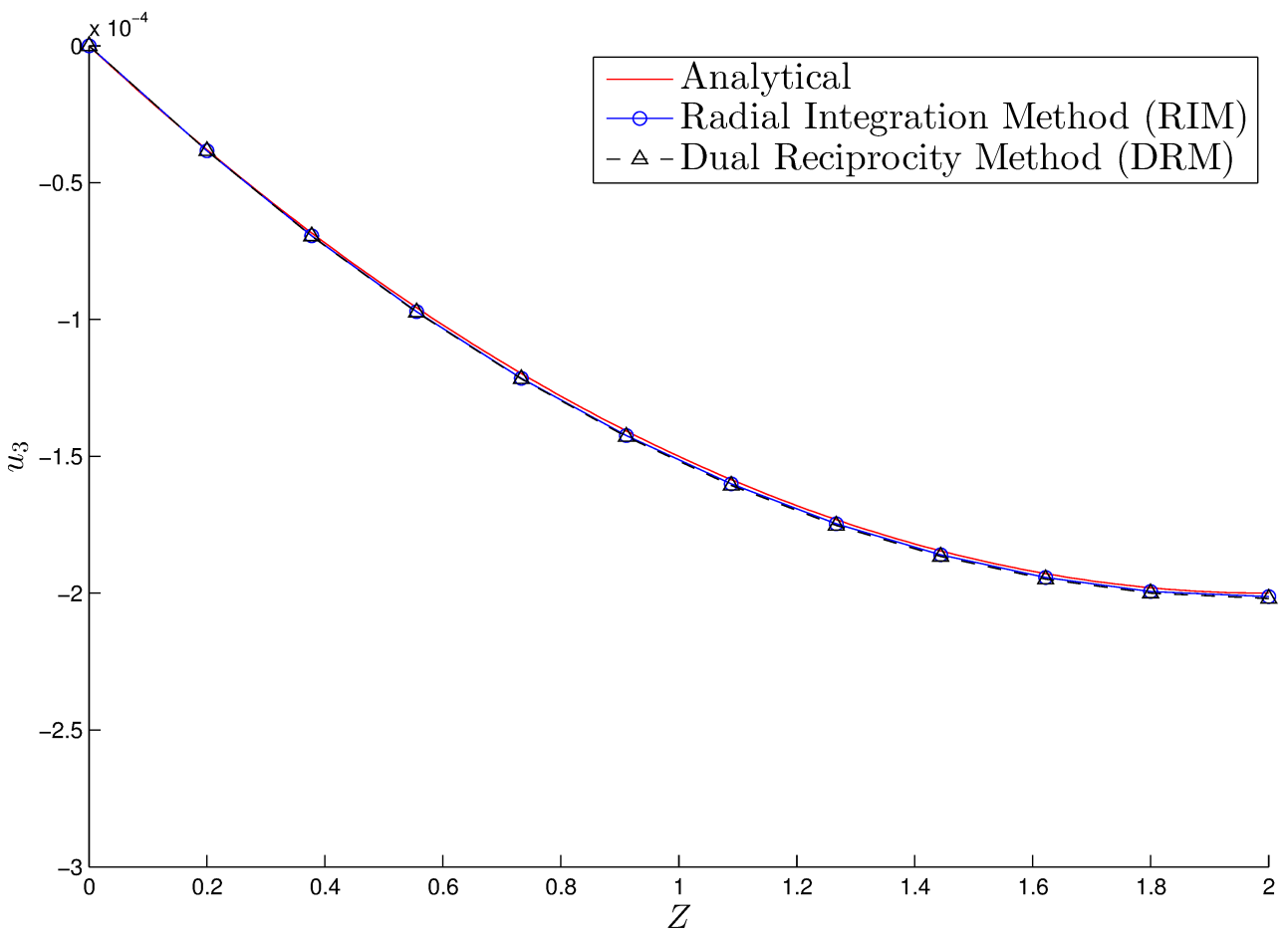

Figure 4.5: Transverse displacement comparison. 
Table 4.1: Displacements $u_{3}$ for DRM, RIM and comparison to the analytical solution.

\begin{tabular}{cccc}
\hline$Z$ & Analytical & DRM (\%Error) & RIM (\%Error) \\
\hline 0.200 & $0.380 \mathrm{E}-4$ & $0.384 \mathrm{E}-4(1.05 \%)$ & $0.384 \mathrm{E}-4(1.05 \%)$ \\
0.378 & $0.684 \mathrm{E}-4$ & $0.696 \mathrm{E}-4(1.75 \%)$ & $0.695 \mathrm{E}-4(1.61 \%)$ \\
0.556 & $0.957 \mathrm{E}-4$ & $0.974 \mathrm{E}-4(1.78 \%)$ & $0.972 \mathrm{E}-4(1.57 \%)$ \\
0.733 & $0.120 \mathrm{E}-3$ & $0.122 \mathrm{E}-3(1.67 \%)$ & $0.122 \mathrm{E}-3(1.67 \%)$ \\
0.911 & $0.141 \mathrm{E}-3$ & $0.143 \mathrm{E}-3(1.42 \%)$ & $0.142 \mathrm{E}-3(0.71 \%)$ \\
1.089 & $0.159 \mathrm{E}-3$ & $0.161 \mathrm{E}-3(1.26 \%)$ & $0.160 \mathrm{E}-3(0.63 \%)$ \\
1.267 & $0.173 \mathrm{E}-3$ & $0.175 \mathrm{E}-3(1.16 \%)$ & $0.175 \mathrm{E}-3(1.16 \%)$ \\
1.444 & $0.185 \mathrm{E}-3$ & $0.187 \mathrm{E}-3(1.08 \%)$ & $0.186 \mathrm{E}-3(0.54 \%)$ \\
1.622 & $0.193 \mathrm{E}-3$ & $0.195 \mathrm{E}-3(1.04 \%)$ & $0.194 \mathrm{E}-3(0.52 \%)$ \\
1.800 & $0.198 \mathrm{E}-3$ & $0.200 \mathrm{E}-3(1.01 \%)$ & $0.199 \mathrm{E}-3(0.51 \%)$ \\
\hline
\end{tabular}

Good agreement was observed when the results using the DRM and the RIM were compared to the analytical solution. The RIM showed to be relatively more accurate with errors of $(0.51 \%-1.67 \%)$, while the DRM gave errors of $(1.01 \%-1.78 \%)$. It is worth to point out that as body forces are known, the integration by the RIM is straightforward and no radial basis function was needed.

\subsubsection{Example B: Body forces on multi-domain anisotropic materials}

In the present example the multi-domain approach is tested for anisotropic materials when subjected to body forces such as gravity. As in Example A, this is an static problem since no dependence on time is considered. The DRM is used for the domain integral transformation. The geometry and boundary conditions are the same as in the previous example. The material is generally anisotropic and was obtained by successive rotations of the material axes of an alpha-quartz crystal. The global Cartesian axes $x_{1}, x_{2}$ and $x_{3}$ were rotated by $30^{\circ}, 45^{\circ}$ and $60^{\circ}$ clockwise, respectively. These successive rotations yield a fully populated stiffness matrix, which can be observed in Equation (3.59). The elastic compliance matrix, $a_{i j}$, can be obtained from the inverse of $C_{i j}$, 


$$
\mathbf{a}=\left[\begin{array}{cccccc}
99.4 & -20.5 & 3.0 & -0.6 & -30.4 & 32.9 \\
-20.5 & 109.6 & 5.6 & 1.8 & 8.9 & -43.1 \\
3.0 & 5.6 & 77.9 & -10.6 & -3.3 & -1.3 \\
-0.6 & 1.8 & -10.6 & 325.0 & -20.3 & -21.1 \\
-30.4 & 8.9 & -3.3 & -20.3 & 274.6 & -16.0 \\
32.9 & -43.0 & -1.3 & -21.0 & -16.0 & 205.8
\end{array}\right] \times 10^{-13} \mathrm{GPa}^{-1}
$$

The bar was modelled with a single region and with the multi-domain approach. For the single region, a total of 40 quadratic discontinuous elements and 320 physical nodes were created to model the problem. Moreover, 10 internal nodes were generated along its axis $(0,0,-Z)$. For the multi-domain approach, a total of 10 regions were created, resulting in 160 quadratic discontinuous elements and 1280 physical nodes. Three internal points per region were also considered for comparison purposes. Numerical results were compared to the analytical solution obtained by Lekhnitskii (1963). The displacements $u, v$ and $w$ are evaluated as,

$$
\begin{gathered}
u=\gamma g\left[-0.5 a_{35} z^{2}+\left(a_{13} x+0.5 a_{36} y\right)(l-z)\right] \\
v=\gamma g\left[-0.5 a_{34} z^{2}+\left(a_{23} y+0.5 a_{36} x\right)(l-z)\right] \\
w=\gamma g\left[0.5\left(a_{13} x^{2}+a_{23} y^{2}+a_{36} x y\right)+\left(a_{34} y+a_{35} x\right) l+0.5 a_{33} z(2 l-z)\right],
\end{gathered}
$$

where $x, y$ and $z$ are the nodal coordinates of the points to be analysed. From Equation (4.30), it can be observed that cross sections do not remain plane, but takes the shape of a second-degree polynomial surface. Likewise, in the case of general anisotropy, the axis become curved. Results for the displacements are shown in Figure 4.6. 

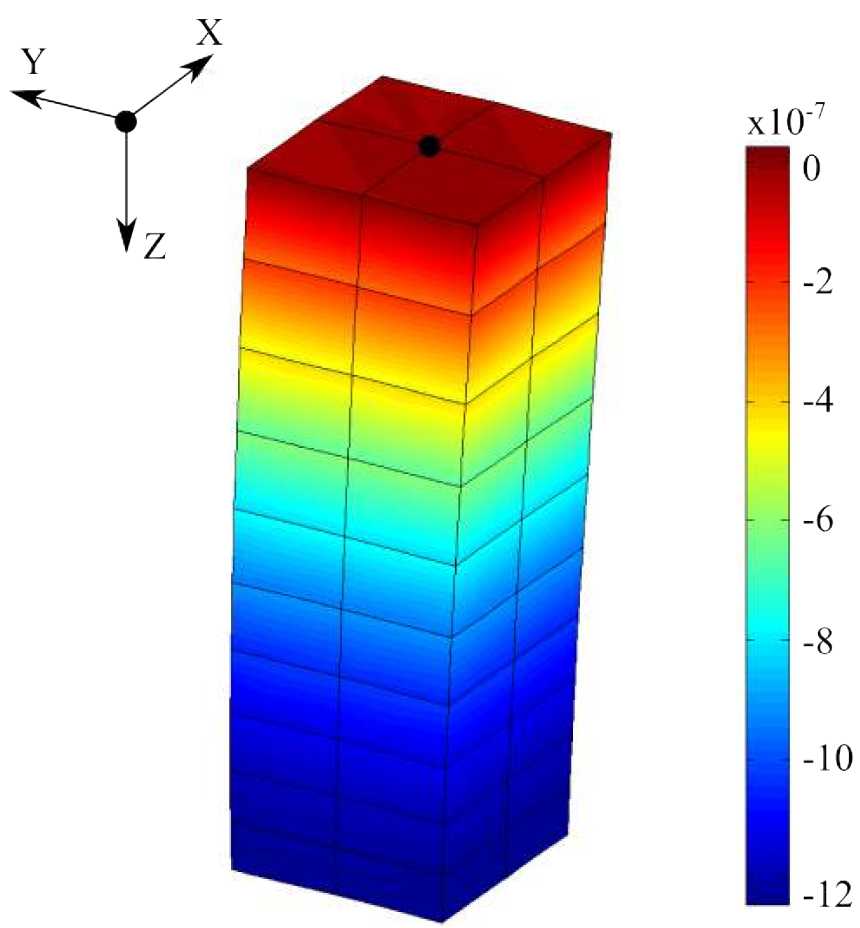

Figure 4.6: Displacement in $x$.

The comparisons to the analytical solution are shown in Figures 4.7, 4.8 and 4.9 for geometrical axes $x, y$ and $z$, respectively. 


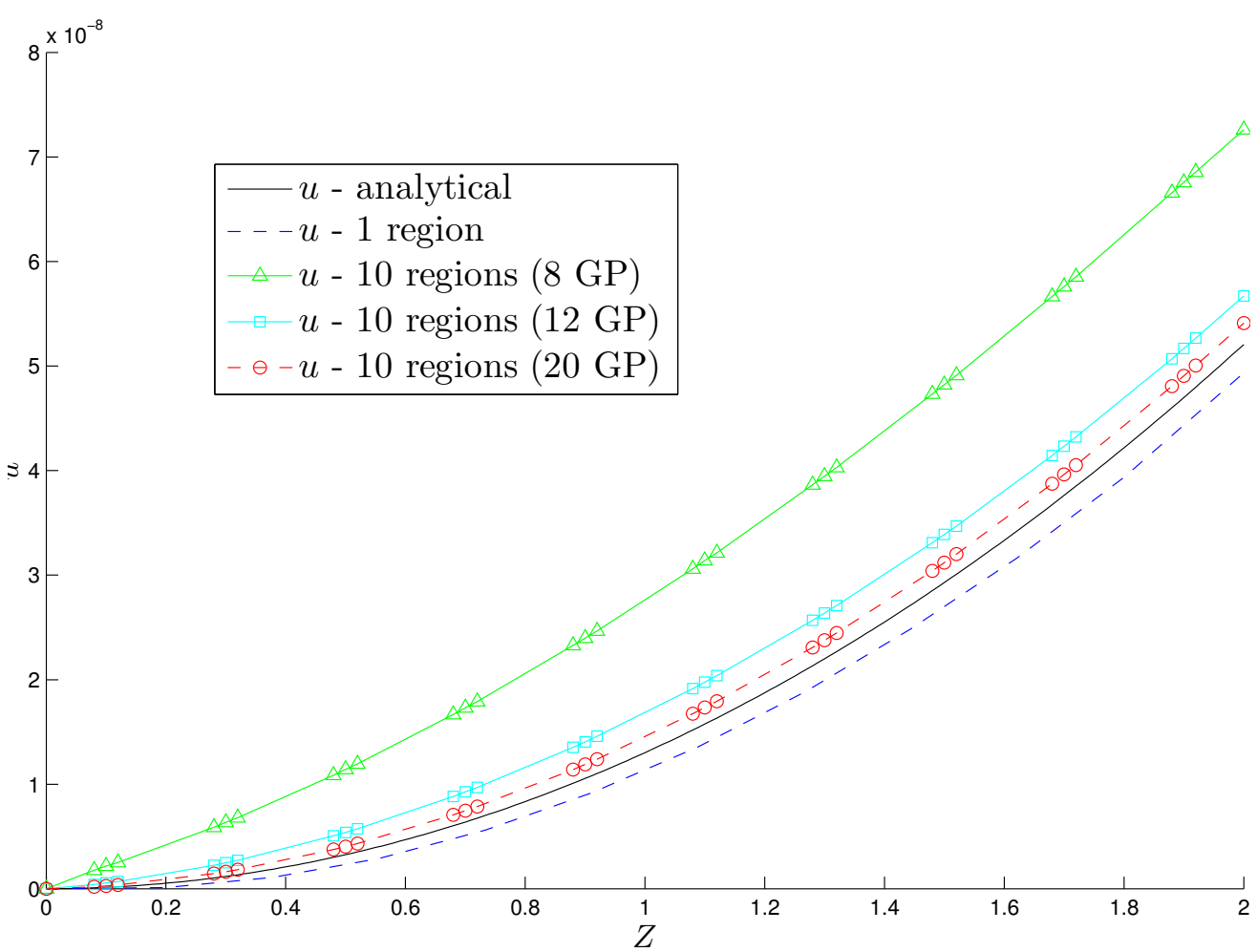

Figure 4.7: $u$ displacement along the $x$ axis.

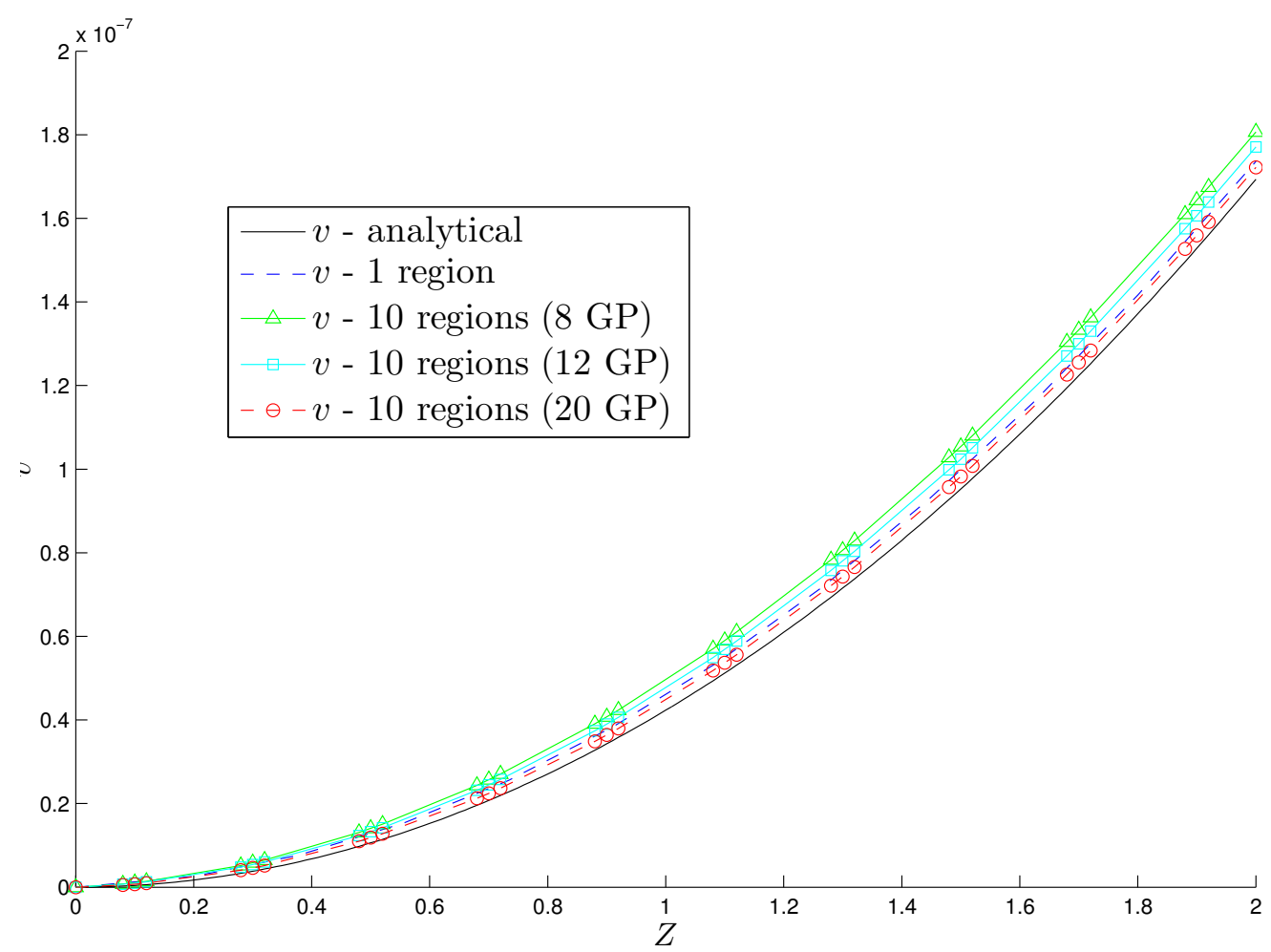

Figure 4.8: $v$ displacement along the $y$ axis. 


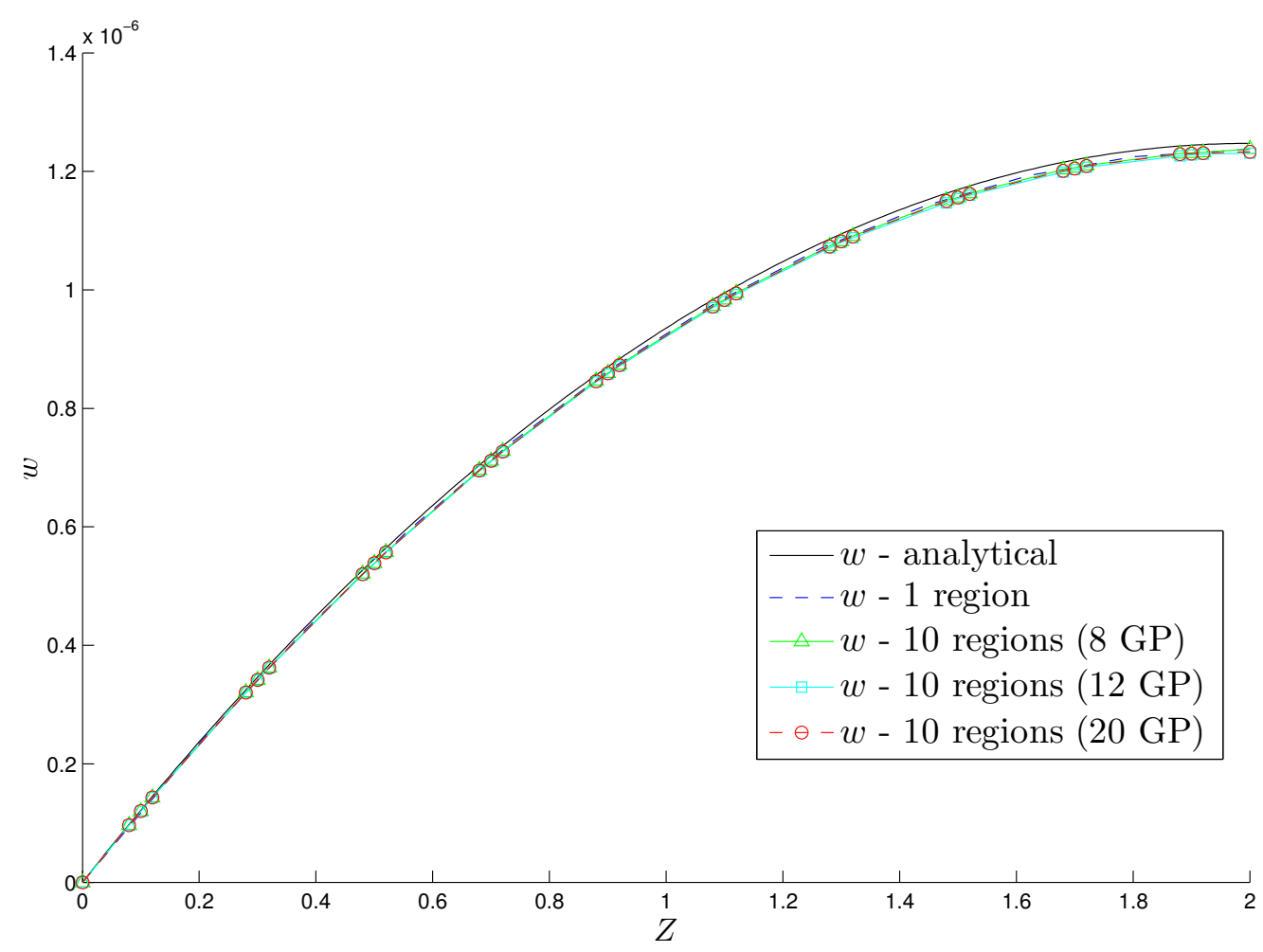

Figure 4.9: $w$ displacement along the $z$ axis.

Results, in general, are in good agreement with the analytical solution proposed by Lekhnitskii (1963). However, it is worth to point out that, for this problem, in order to maintain the accuracy of the anisotropic response it is necessary to increase the number of the Gauss points in the singular and non-singular BEM integration scheme when increasing the number of regions. For the case of 10 regions, $12 \times 12$ Gauss points per integrated section were needed to yield an acceptable accuracy for the $u$ displacements in the $x$ coordinate axis. For this case, an error of $8.4 \%$ was obtained, while the error for the single region ( $8 \times 8$ Gauss points) was $5.2 \%$. The error decreases as the number of Gauss points increases; for $u$ displacements, 20x20 Gauss points yield to an error of 3.9\%. For $v$ and $w, 12 \times 12$ Gauss points were enough to yield good results, error of $4.5 \%$ and $1.3 \%$, respectively.

\subsubsection{Example C: Transient dynamic analysis - Heaviside step load}

In this example the direct approach in the transient dynamic analysis is tested. The geometric model used is a thin isotropic bar, shown in Figure 4.10, with elasticity modulus $E=200000 \mathrm{~N} / \mathrm{mm}^{2}$, Poisson's ratio $\nu=0$, and mass density $\rho=7850 \mathrm{~kg} / \mathrm{m}^{3}$, (Gaul 
et al., 2003). The bar is clamped at $X_{1}=0 \mathrm{~mm}$ and is initially at rest. A Heaviside step load $\bar{t}_{1}(t)=100 \mathrm{~N} / \mathrm{mm}^{2}$ is applied at $X_{1}=100 \mathrm{~mm}$. The propagation of the elastic waves in the $X_{1}$ coordinate is calculated with the DRM and the RIM.

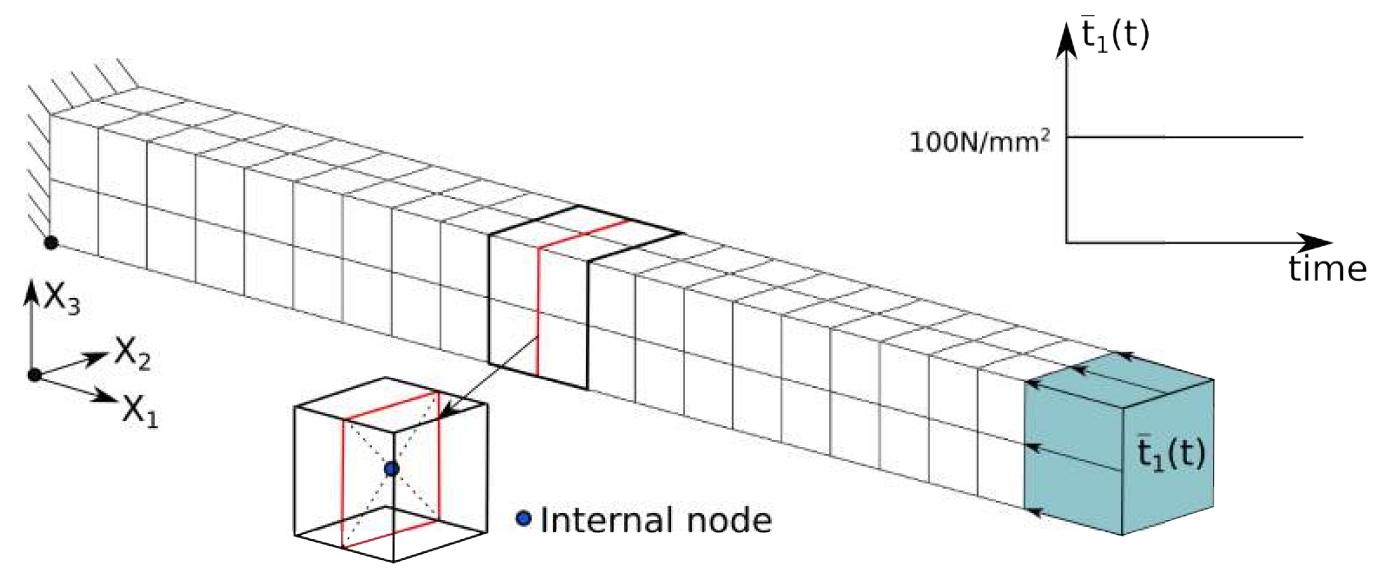

Figure 4.10: Geometry, boundary conditions and mesh of the isotropic bar.

The analytical solution of the transient undamped response of a prismatic bar to an axial load, presented by Clough and Penzien (2003), is

$$
u(x, t)=\sum_{n=1}^{\infty} \bar{\phi}_{n}(x) Y_{n}(t)
$$

where $\bar{\phi}_{n}(x)$ are the mode shapes and $Y_{n}(t)$ is the generalized-coordinate response, calculated as,

$$
\begin{gathered}
\bar{\phi}_{n}(x)=\sin \left[(2 n-1) \frac{\pi x}{2 L}\right] \\
Y_{n}(t)= \pm \frac{2 P_{0}}{m L \omega_{n}^{2}}\left(1-\cos \omega_{n} t\right) \quad\left\{\begin{array}{l}
+n=\text { even no. } \\
-n=\text { odd no. }
\end{array}\right.
\end{gathered}
$$

where $L$ is the bar length, $P_{0}$ is the load and $m$ is the mass per unit length. The circular frequency, $\omega_{n}$, is evaluated as, 


$$
\omega_{n}=(2 n-1) \frac{\pi}{2} \sqrt{\frac{E A}{m L^{2}}} \quad n=1,2, \ldots
$$

Finally, substituting Equation (4.32) and Equation (4.33) into Equation (4.31), the expression for the transient displacement response results in, (Clough and Penzien, 2003),

$$
u(x, t)=\frac{8 P_{0} L}{\pi^{2} E A} \sum_{n=1}^{\infty}\left\{ \pm\left(\frac{1-\cos \omega_{n} t}{(2 n-1)^{2}}\right) \sin \left[\frac{(2 n-1)}{2} \frac{\pi x}{L}\right]\right\}
$$

The bar was modelled with 168 quadratic discontinuous elements and 1344 physical nodes for the DRM. The computational requirements for applying the RIM to unknown body forces are largely higher than for the DRM. For this discretization, e.g., the DRM takes approximately 15 minutes for generating all BEM matrices, while the RIM was estimated to take approximately 10 days, 12 hours and 35 minutes for the same procedure. Thus, a new discretization for applying the RIM was necessary, as can be seen at Figure 4.11.

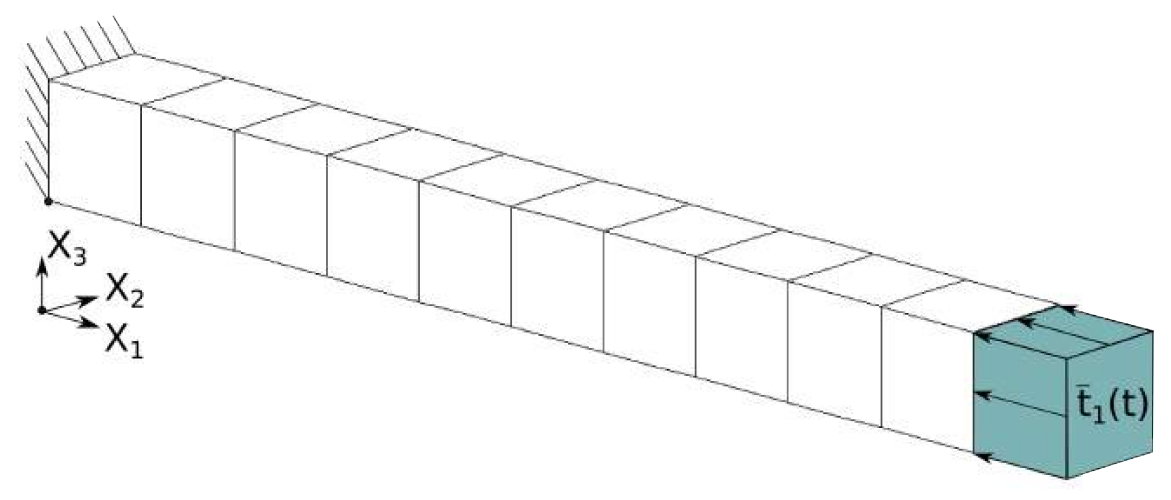

Figure 4.11: Mesh for the RIM analysis.

For the RIM discretization 42 quadratic discontinuous elements and 336 physical nodes were created. For both discretizations 11 internal points equally distributed at $\left(0.5,0.5, X_{1}\right)$ were considered. Nevertheless, even a poor discretization for the RIM gave more accurate results than the DRM when results were compared to the analytical solution. A smaller time step also was used when the RIM was applied. The displacement response $u_{1}(t)$ at $(50,5,5) \mathrm{mm}$ for both methods and the analytical solution are shown in Figure 4.12. 


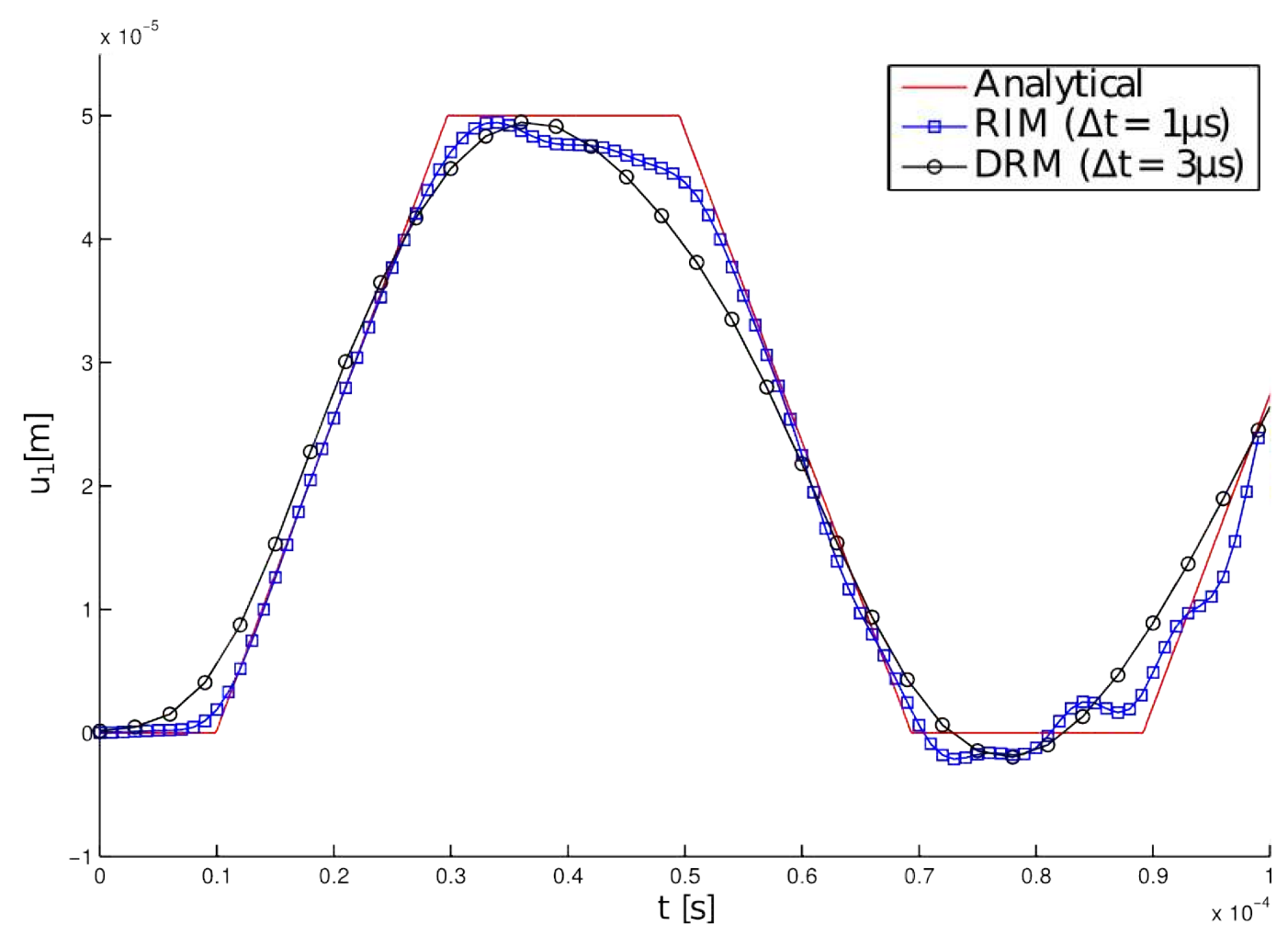

Figure 4.12: $u_{1}(t)$ displacement comparison at $(50,5,5) \mathrm{mm}$.

The purpose of showing different time steps on the results for the DRM and the RIM, Figure 4.12, is to show the best possible result for each method. Smaller time steps will not produce acceptable displacement results for the DRM, e.g., while bigger time steps will produce too smoothed results. Thus, it is observed that for the same mesh discretization and time intervals the RIM is more accurate than the DRM. Even for coarser meshes and small time intervals the RIM kept stable when compared to the DRM. However, a further convergence analysis is always recommended for each problem. Small time intervals can lead to a more accurate response, but increase the risk of instability, whereas larger values tend to yield inaccurate, smooth results. For a more complete analysis of stability on time integration refer to (GAUL et al., 2003). For now on the RIM will not be applied due to its high computational requirements when dealing with high number of degrees of freedom.

\subsubsection{Example D: Transient dynamic analysis - ramp load}

As the last example, the direct approach in the transient analysis is tested, but, for a ramp load. The geometric model is also a thin isotropic bar, shown in Figure 4.13, with elasticity 
modulus $E=200000 \mathrm{~N} / \mathrm{mm}^{2}$, Poisson's ratio $\nu=0$, and mass density $\rho=7850 \mathrm{~kg} / \mathrm{m}^{3}$, (Gaul et al., 2003). The bar is clamped at $X_{1}=0 \mathrm{~mm}$ and is initially at rest. A ramp load $\bar{t}_{1}(t)=100 \mathrm{~N} / \mathrm{mm}^{2} t$ is applied at $x_{1}=100 \mathrm{~mm}$. The propagation of the elastic waves in the $X_{1}$ coordinate is calculated with the DRM.

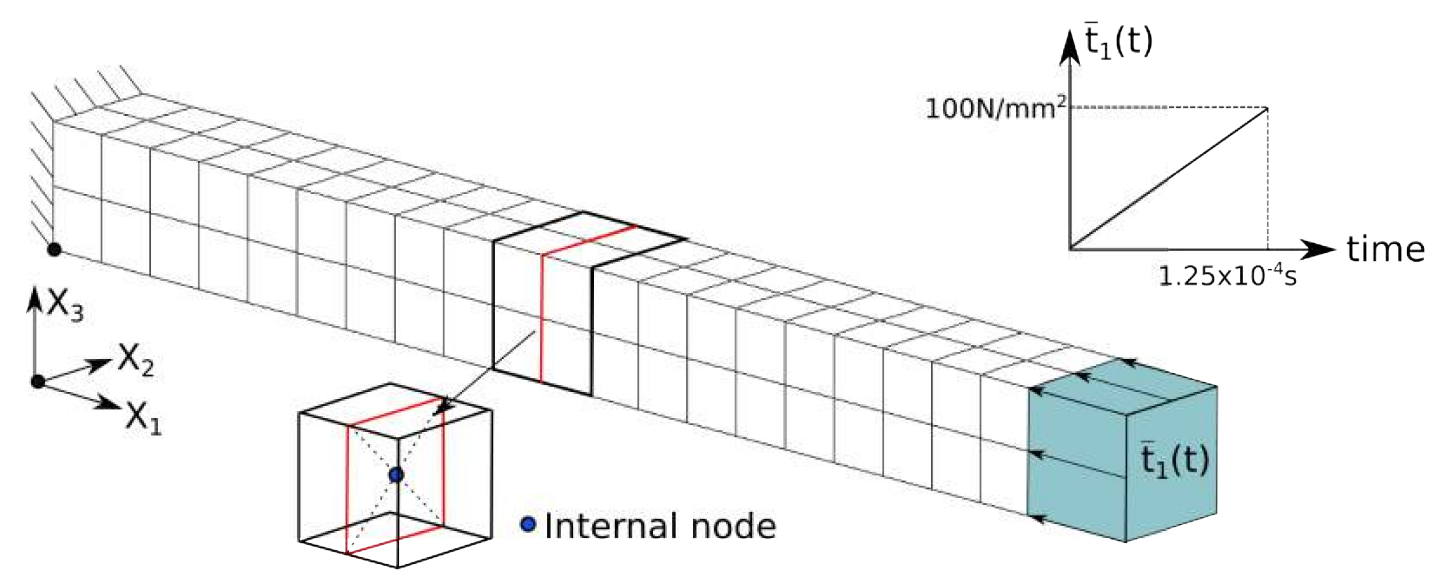

Figure 4.13: Geometry, boundary conditions and mesh of the isotropic bar.

The analytical solution for a transient ramp axial load follow the same procedure as the step load shown previously. However, with different boundary conditions for the equation of motion. The expression for the transient displacement response for this case is, (Clough and Penzien, 2003),

$$
u(x, t)=\frac{8 P_{0} L}{\pi^{2} E A} \sum_{n=1}^{\infty}\left\{ \pm\left(\frac{t-\sin \omega_{n} t}{\omega_{n}(2 n-1)^{2}}\right) \sin \left[\frac{(2 n-1)}{2} \frac{\pi x}{L}\right]\right\}
$$

with the same variables previously described at Example B. The bar was modelled with 168 quadratic discontinuous elements and 1344 physical nodes. Moreover, 11 internal points equally distributed at $\left(0.5,0.5, X_{1}\right)$ were also considered. The transient analysis consisted of a total time of $1.25 \times 10^{-4} \mathrm{~s}$, which represented 50 time steps with a time interval of $\Delta t=2.5 \mu \mathrm{s}$. The displacement response $u_{1}(t)$ at $(50,5,5) \mathrm{mm}$ and the analytical solution are shown in Figure 4.14. 


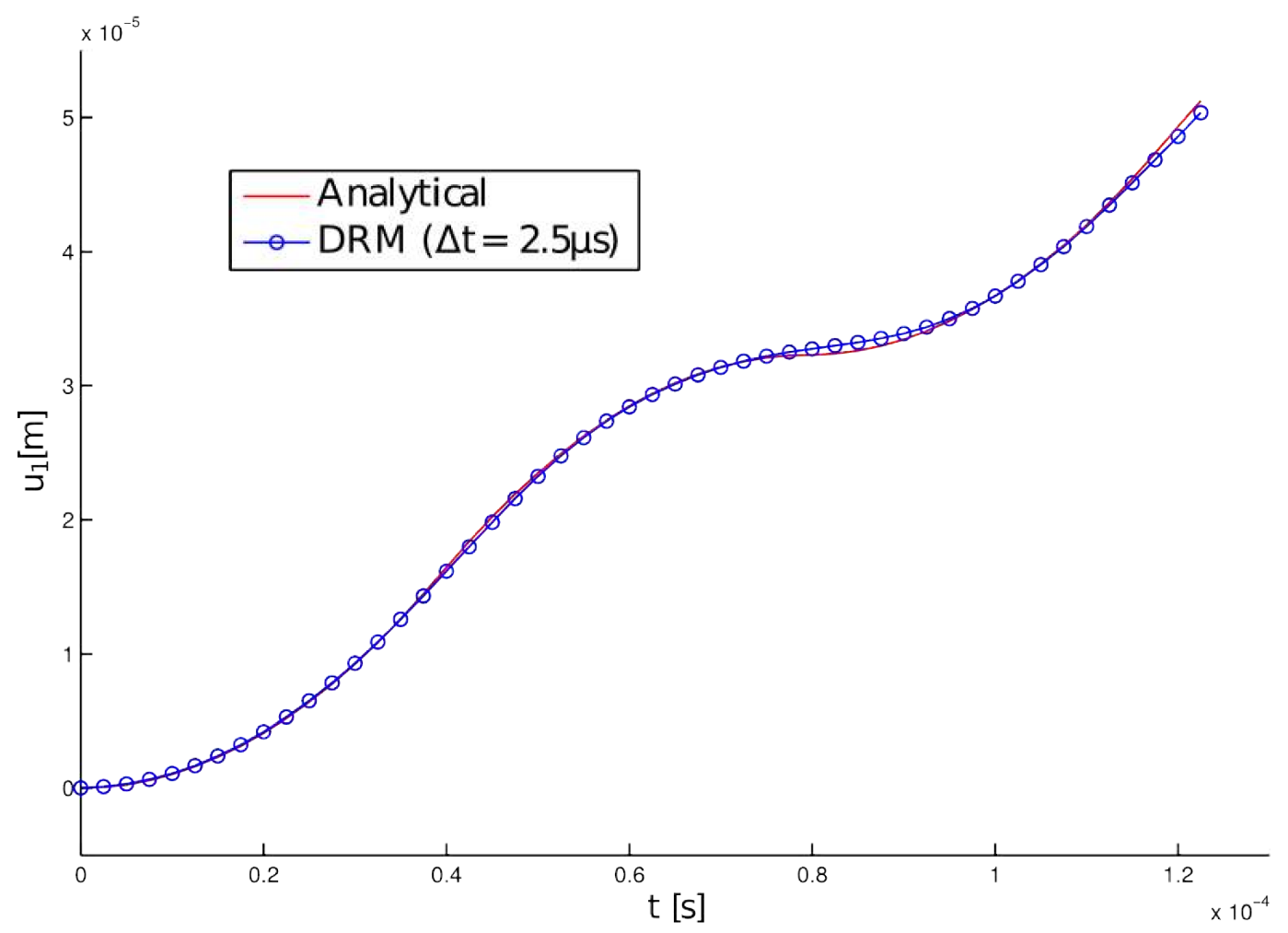

Figure 4.14: $u_{1}(t)$ displacement comparison at $(50,5,5) \mathrm{mm}$.

Good agreement is observed when the DRM was compared to the analytical solution. A maximum error of $1.88 \%$ was found at $t=8.5 \times 10^{-5} \mathrm{~s}$. As explained previously, small time intervals lead to a more accurate response, but increases the risk of instability. For this example, a value of $\Delta t=2.5 \mu$ s was found to be a good choice to achieve both accuracy and stability of the transient displacement response. 


\section{Fast BEM approach}

\subsection{Introduction}

Having dealt with the conventional BEM analysis in the previous three chapters, a fast BEM approach will now be studied. The main objective is to reduce the storage requirements and to speed up the matrix generation process. The steps involved are outlined first, the hierarchical clustering is illustrated in which the construction of clusters as well as the process of finding admissible blocks are shown. Then, the low rank approximation, by means of the Adaptive Cross Approximation (ACA), is presented. The ACA is applied to a 2D multiple inclusion potential problem as a demonstration of the scheme. Finally, a illustrative example showing the fast BEM process and the numerical results obtained are presented to validate the formulation.

\subsection{Fast BEM approach}

The objective of the Fast BEM approach is to reduce the computational storage requirements as well as to speed up the time required to complete all matrix operations. The Fast BEM approach is based on the hierarchical approximation of large dense matrices arising from some generating function having diagonal singularity, and it consists of three basic steps (Kurz et al., 2007):

(i) construction of clusters;

(ii) finding of possible admissible blocks; and

(iii) low rank approximation of admissible blocks.

The first two steps, (i) and (ii), are generally denominated as hierarchical clustering. The low rank approximation will be treated with the Adaptive Cross Approximation (ACA). Both, are briefly described below. 


\subsubsection{Hierarchical clustering}

The main reference in this section is (BENEDETTI et al., 2011), where a study of the use of hierarchical clustering and low rank approximations applied to large-scale 3D elastic problems is assessed. A formal definition and description of hierarchical matrices can also be found in (HACKBUSCH; HACKBUSCH AND NOWAK, 1999; 2000).

The construction of clusters was implemented based on the algorithm showed in (KURZ et al., 2007). First, the mass and centre of each cluster are stored, and the covariance matrix $C$ of the cluster is obtained, as follows,

$$
C=\sum_{k=1}^{n} g_{k}\left(x_{k}-X\right)\left(x_{k}-X\right)^{\mathrm{T}}
$$

where $n$ is the number of elements of the cluster, $g_{k}$ is the element length and $X$ is the centre of the cluster. The eigenvector $v_{1}$ corresponding to the largest eigenvalue of $C$ shows the direction of the largest extension of the cluster. A separation plane goes through the centre $X$ of the cluster and is orthogonal to $v_{1}$. This algorithm is applied recursively to the sons, dividing them into cluster with approximately the same number of elements. For example, Figure 5.1 shows the first two levels of separation of an exhaust manifold (KURZ et al., 2007). This algorithm will be applied recursively to the sons until they contain less than or equal to some prescribed number $n_{\min }$ of elements.
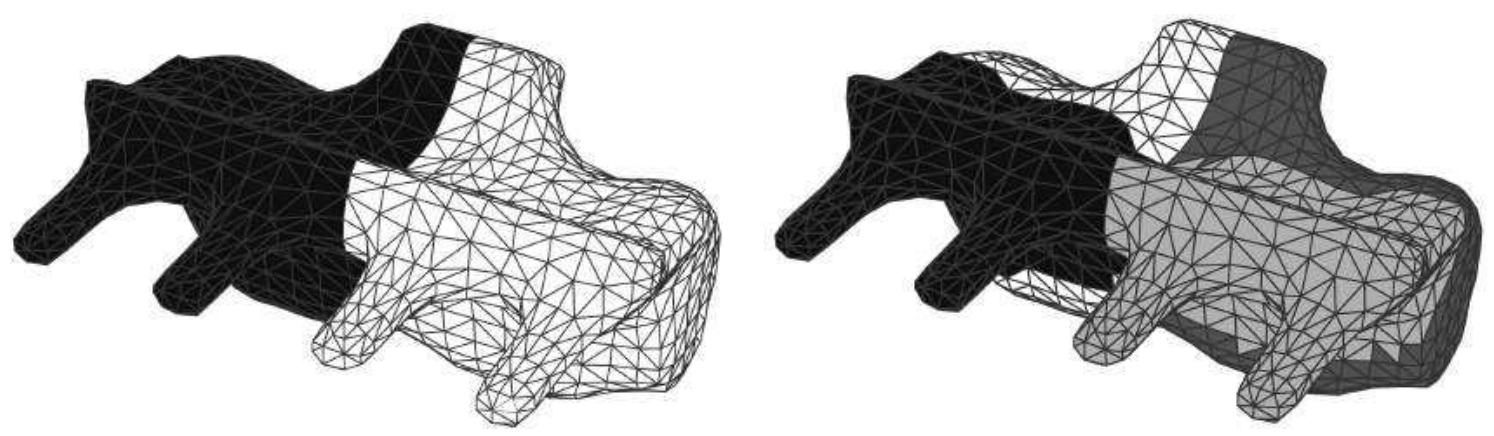

Figure 5.1: Clusters created in the first two levels (KURZ et al., 2007).

Next, cluster pairs which are geometrically well separated are identified and regarded as admissible cluster pairs, as can be seen in Figure 5.2. An appropriate admissibility criterion is the following simple geometrical condition. 
A pair of clusters $\left(C l_{x}, C l_{y}\right)$ is admissible if, (Kurz et al., 2007),

$$
\min \left(\operatorname{diam}\left(C l_{x}\right), \operatorname{diam}\left(C l_{y}\right)\right) \leq \eta \operatorname{dist}\left(C l_{x}, C l_{y}\right)
$$

where $\eta$ is called the admissibility parameter. This parameter influences the number of admissible blocks and the convergence speed of the ACA (Borm et al., 2003). As $\eta$ decreases, the distance has to be higher in order to account for the admissibility criterion, thus, implying in a more restrictive criterion. This parameter influences the number of admissible blocks on one hand, and the convergence speed of the adaptive approximation of low rank blocks on the other hand (BORM et al., 2003). A full study of this parameter was assessed by Benedetti et al. (BENEDETTI et al., 2011). They showed that the choice of $\eta$ directly affects the quality of the ACA-generated matrix and a good choice of this parameter results in a matrix closer to the optimal matrix produced by the coarsening procedure. This fact was also justified by the reduction in the number of blocks.

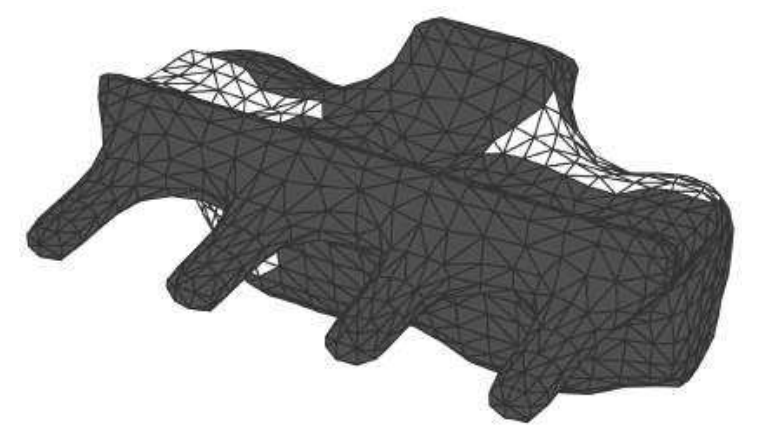

Figure 5.2: Admissible cluster pair (KURZ et al., 2007).

In the present work, the actual diameters and the distance between two clusters were calculated and no approximation, as suggested by (BENEDETTI et al.; KURZ et al.; GRASEDYCK; GIEBERMAN, 2011; 2007; 2005; 2001), was made. Approximations are easily computable; however, it would result in a more restrictive criterion. Once the clusters are defined and all admissible blocks are detected, the Adaptive Cross Approximation (ACA) is used to approximate by low rank these blocks. 


\subsubsection{Adaptive Cross Approximation - ACA}

In BEM analysis, the kernels of the BIE are computed and they are the coefficients of the system matrices. In a potential theory problem, the kernels are the potential and flux fundamental solutions, while in an elasticity problem, the kernels are the displacement $U_{j k}$ and traction $T_{j k}$ fundamental solutions. They both depend upon the positions of the source point $\mathbf{d}$ and the field point $\mathbf{x}$. The kernels $U_{j k}(\mathbf{d}, \mathbf{x})$ and $T_{j k}(\mathbf{d}, \mathbf{x})$ are two-point asymptotic smooth functions and singular when $\mathbf{d}=\mathbf{x}$. These functions can be approximated by a sum of products of two functions $u_{i}(\mathbf{d})$ and $v_{i}(\mathbf{x})$,

$$
\kappa(\mathbf{d}, \mathbf{x})=\sum_{i=1}^{k} u_{i}(\mathbf{d}) v_{i}(\mathbf{x})+R_{\kappa}(\mathbf{d}, \mathbf{x})
$$

where $\kappa$ can be either $u_{j k}$ or $t_{j k}, R_{\kappa}(\mathbf{d}, \mathbf{x})$ is the error of the approximation and $\left|R_{\kappa}(\mathbf{d}, \mathbf{x})\right| \leq \varepsilon_{\kappa}$ with $\varepsilon_{\kappa} \rightarrow 0$ for $k \rightarrow 0$.

The existence of low rank approximants is based on the asymptotic smoothness of the kernel functions, i.e., on the fact that kernels $U_{i j}$ and $T_{i j}$ are singular only when the source and field points are coincident, when $\mathbf{x}=\mathbf{y}$ (Bebendorf, 2000; Bebendorf and Rjasanow, 2003; Grasedyck, 2005). This is a sufficient condition for the existence of low rank approximants. A low rank block $M$ of size $m \times n$ has the following representation

$$
M_{k}=\sum_{i=1}^{k} a_{i} \cdot b_{i}^{T}=A \cdot B^{\mathrm{T}}
$$

where $A$ is a matrix of size $m \times k$ and $B$ is a matrix of size $n \times k$. For admissible blocks, $k$ is low and the representation shown in Equation (5.4) requires the storage of $(m+n) k$ real numbers instead of the of the $m \times n$ original block, speeding up the matrix-vector product of the corresponding block. For a detailed analysis, refer to (BORM et al.; GRASEDYCK AND HACKBUSCH, 2003; 2003).

The approximation block $M_{k}$ satisfies the relation $\left\|M-M_{k}\right\|_{F} \leq \varepsilon\|M\|_{F}$, where $\|\cdot\|_{F}$ represents the Frobenius norm and $\varepsilon$ is the prescribed relative error. The low rank blocks are constructed by computing and storing only some of the original blocks entries. Such entries 
allow the representation presented in equation (5.4) through suitable algorithms, namely, the Adaptive Cross Approximation (ACA). The ACA algorithm allows the selected collocation matrix accuracy $\varepsilon$ to be reached adaptively. In contrast to other methods like FMM, for example, the kernel of the integral operator for evaluating the ACA approximants is not replaced by other terms. Moreover, the calculated rows and columns are sufficient to evaluate the accuracy of the approximation without calculating all the coefficients in the whole block. One of main advantages of the ACA is that block entries can be easily evaluated by the existing code, whereas methods like the FMM require a complete re-coding.

The algorithm starts evaluating a random row $M^{\mathrm{T}} e_{k_{i+1}}$ and finding the location $(k+1, l+$ 1) of its pivot (maximum absolute value of the row coefficients). If the pivot is non-zero, the entire column $M e_{l_{i+1}}$ is evaluated, scaled by specific product and divided by the pivot. Hence, the calculated entries are checked with a stop criterion. If this criterion is not fulfilled, a new row $M^{\mathrm{T}} e_{k_{i+2}}$ in the same column is generated and the entire process is thus repeated until the criterion is satisfied. A record of the already calculated entries avoids the need to find the same previous pivots. The algorithm, as defined by (KURZ et al., 2007), is outlined below,

\section{ACA algorithm:}

1. Initialisation

$$
S_{0}=0, \quad I=\varnothing, \quad c=0 \in \Re^{N},
$$

\section{Recursion}

2.1 Choice of the next (not yet generated) row

$$
k_{i+1}=\min \{k: k \notin I\}, \quad I=I \cup\left\{k_{i+1}\right\},
$$

or stop if all rows are generated, i.e. $I=1, \ldots, N$,

2.2 Generation of the row

$$
a=M^{\mathrm{T}} e_{k_{i+1}},
$$

2.3 Row of the residues and the pivot column

$$
\begin{gathered}
R_{i}^{\mathrm{T}} e_{k_{i+1}}=a-\sum_{m=1}^{i}\left(u_{m}\right)_{k_{i+1}} v_{m} \\
l_{i+1}=\operatorname{ArgMax}\left|\left(R_{i}\right)_{k_{i+1} l}\right|
\end{gathered}
$$

2.4 Test 


$$
\text { if } \operatorname{Max}\left|\left(R_{i}\right)_{k_{i+1} l}\right|=0 \text { then go to } 2.1 \text {. }
$$

2.5 Normalizing constant

$$
\gamma_{i+1}=\left(\left(R_{i}\right)_{k_{i+1} l_{i+1}}\right)^{-1}
$$

2.6 Generation of the column, update of the control vector

$$
a=M e_{l_{i+1}}, \quad c=c+|a|,
$$

2.7 Column of the residues and the pivot row

$$
\begin{gathered}
R_{i} e_{l_{i+1}}=a-\sum_{m=1}^{i}\left(v_{m}\right)_{l_{i+1}} u_{m}, \\
k_{i+2}=\operatorname{ArgMax}\left|\left(R_{i}\right)_{k l_{i+1}}\right|,
\end{gathered}
$$

2.8 New vectors

$$
u_{i+1}=\gamma_{i+1} R_{i} e_{l_{i+1}}, \quad v_{i+1}=R_{i}^{\mathrm{T}} e_{k_{i+1}},
$$

2.9 New approximation

$$
S_{i+1}=S_{i}+u_{i+1} v_{i+1}^{\mathrm{T}}
$$

2.10 Recursion

$$
i=i+1 \text {, go to } 2.2 \text {. }
$$

Since the matrix $M$ will not be generated completely the norm of its approximant $S_{i}$ can be used to define a stop criterion. This norm can be computed recursively as follows,

$$
\left\|S_{i+1}\right\|_{F}^{2}=\left\|S_{i}\right\|_{F}^{2}+2 \sum_{m=1}^{i} u_{i+1}^{\mathrm{T}} u_{m} v_{m}^{\mathrm{T}} v_{i+1}+\left\|u_{i+1}\right\|_{F}^{2}\left\|v_{i+1}\right\|_{F}^{2}
$$

An appropriate stop criterion in Step 2.8 is then

$$
\left\|u_{r}\right\|_{F}\left\|v_{r}\right\|_{F} \leq \varepsilon\left\|S_{r}\right\|_{F}
$$

Since the whole matrix $M$ will not be generated while using the ACA algorithm, it is necessary to check the control vector $c$ updated after every column generation for zero components in not yet generated rows. If there is an index $i^{*} \notin I$ with $c_{i^{*}}=0$ then the row $i^{*}$ has 
not yet contributed to the matrix. It can happen that this row contains relevant information and, therefore, it is necessary to set $i=i+1, k_{i+1}=i^{*}$ and to restart the algorithm in Step 2.2. With this trivial modification, the algorithm can be used not only for dense matrices but also for reducible and even for sparse matrices (KURZ et al., 2007). To summarize, ACA allows a substantial reduction in storage requirements, besides speeding-up the matrix operations. This fact allows the possibility of dealing with large-scale problems, such as those encountered in multiscale problems.

\subsection{ACA applied to a multiple inclusion 2D potential theory problem}

The multidomain or multizone BEM approach is the appropriate technique to treat multiple inclusion problems. A potential theory problem will be considered here. The boundaries of the subdomain which represent each distinct region, are discretized into an assembly of elements. After the discretization into subdomains, the coupling can be made by considering the continuity of the potential and equilibrium of the flux at all the interface nodes. Figure 5.3 shows a multidomain potential problem with four inclusions.

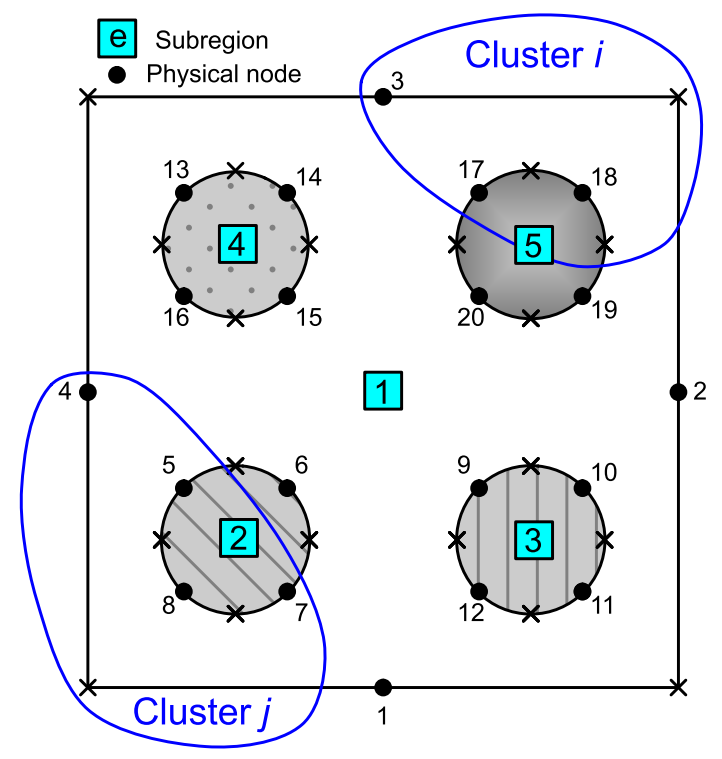

Figure 5.3: Example for the application of ACA in multidomain problems.

In Figure 5.3, subregion one (the physical matrix) has twenty physical nodes and each inclusion (say, fibers) has four physical nodes. However, the overall system matrix is not a 20x20 square matrix. This is because of the coupling of the potential and the potential gradient 
at each interface node between the matrix and the inclusions. In the problem above, there will be 16 (4 inclusions $x 4$ nodes per inclusion) additional unknowns to be determined. Figure 5.4 (a) shows the collocation matrix for this example. $\left[F_{i j}\right]^{e}$ and $\left[G_{i j}\right]^{e}$ represent the contribution in matrices $[F]$ and $[G]$ between subregions $i$ and $j$ in the case where quantities are associated to a specific region $e$. Figure 5.4 (b) shows the contributions for nodes of Cluster $i$ and Cluster $j$ of Figure 5.3. The equations for nodes 3,17 and 18 of Cluster $i$ are represented by rows 3, 17, 18,33 and 34 of the collocation matrix, and, those for nodes 4, 5, 7 and 8 are represented by columns $4,5,7,8,21,23$ and 24 of the collocation matrix, as can be observed in green from Figure 5.4 (b).

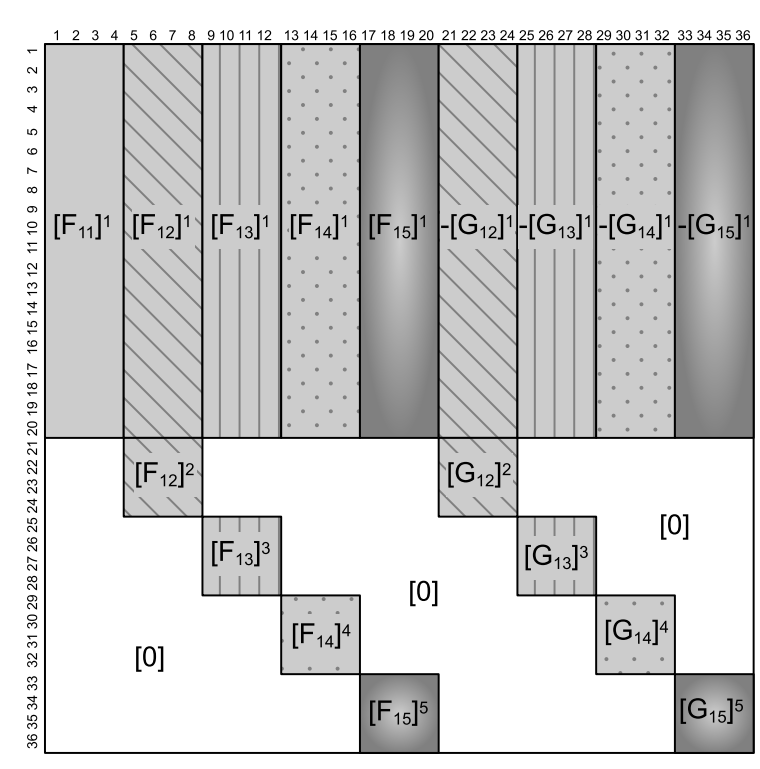

(a)

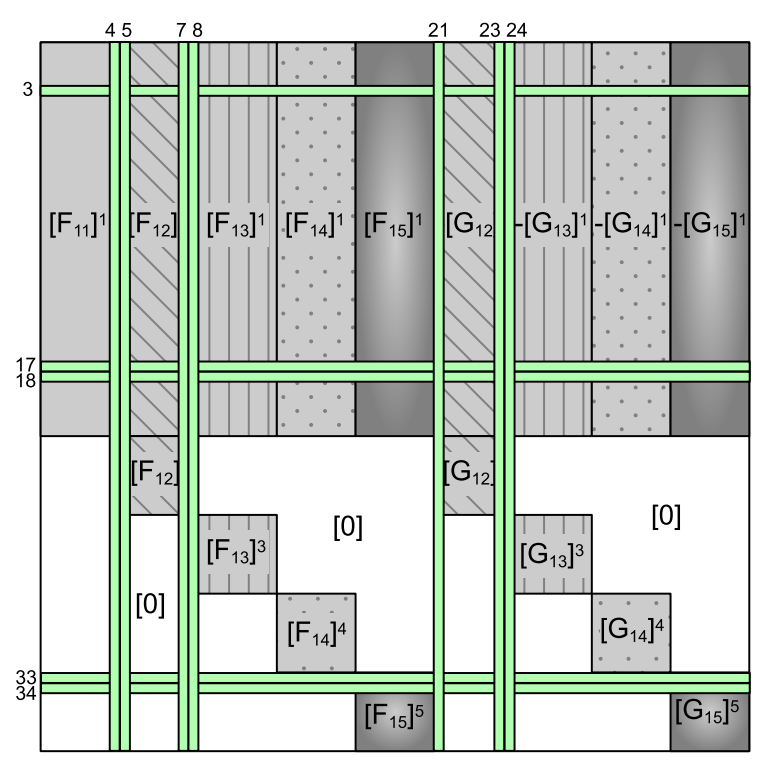

(b)

Figure 5.4: (a) Collocation matrix for the four inclusion example, (b) contributions for physical nodes for the inclusion example

In other words, at each of the interface nodes, there are contributions to additional rows and columns of the system collocation matrix. At an interface node " $n$ ", these contributions will be to $n$ and $\left(n+N_{i}\right)$ rows and columns where $N_{i}$ is the total number of interface nodes in the system. Further, the Adaptive Cross Approximation can be applied if Cluster $i$ and Cluster $j$ meet an admissibility criterion.

Applying the ACA to admissible blocks will cause substantial reduction in storage requirement since the ACA allows the construction of the collocation matrix by computing and storing only few original blocks entries. For example, if in this example the ACA attains the desired accuracy by just computing 3 out of the 5 rows of Cluster $i$, this will imply in 
$2 / 5 \times 100 \%=40 \%$ ( 2 rows will not be calculated) of memory savings for this block. Consequently, there will be less operations to construct the collocation matrix, which will also speed up the entire process.

\subsection{Numerical examples}

Five numerical examples are presented to demonstrate the different aspects of the fast BEM approach. In the first example, it is briefly illustrated with an application to a simple $3 \mathrm{D}$ bar. No numerical results are shown, as this example is presented for didactic purposes. In the second example, the ACA is applied to a multiple inclusion 2D heat conduction problem. Two different types of lattices are analysed and good agreement will be shown for both cases. Then, the ACA is applied to: a problem with an alpha-quartz material in Example C; and to a generally anisotropic material obtained by successive rotations of the stiffness matrix in Example D. Finally, the short fiber-matrix model is presented, tested with an isotropic material (for comparison purposes) and with realistic materials for both, fiber and matrix.

\subsubsection{Example A: Hierarchical clustering in fast BEM}

In this example a simple 3D bar is used to briefly illustrate the hierarchical clustering in a 3D structure. The geometry and elements are shown in Figure 5.5. 


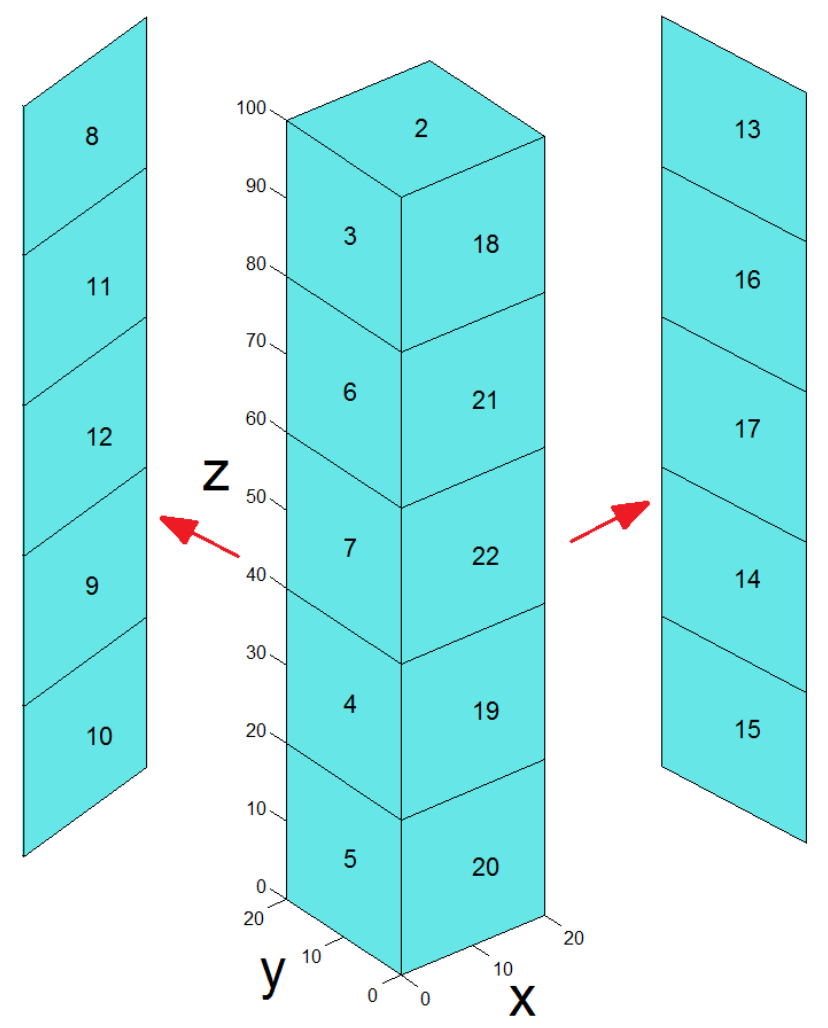

Figure 5.5: Geometry of the bar showing the elements.

As described in Section 5.1, the hierarchical approximation of matrices consists of three steps: Construction of clusters, finding of admissible blocks and low rank approximation of the admissible blocks.

First, clusters are constructed based on the algorithm as used by Kurz et al. (2007). Clusters are created recursively until every cluster has a maximum number of elements $n_{\text {max }}$; for this example a $n_{\max }$ of four is stated. Figure 5.6 shows the cluster division in a cluster tree format, while Figure 5.7 presents the cluster division in a geometrical format. 


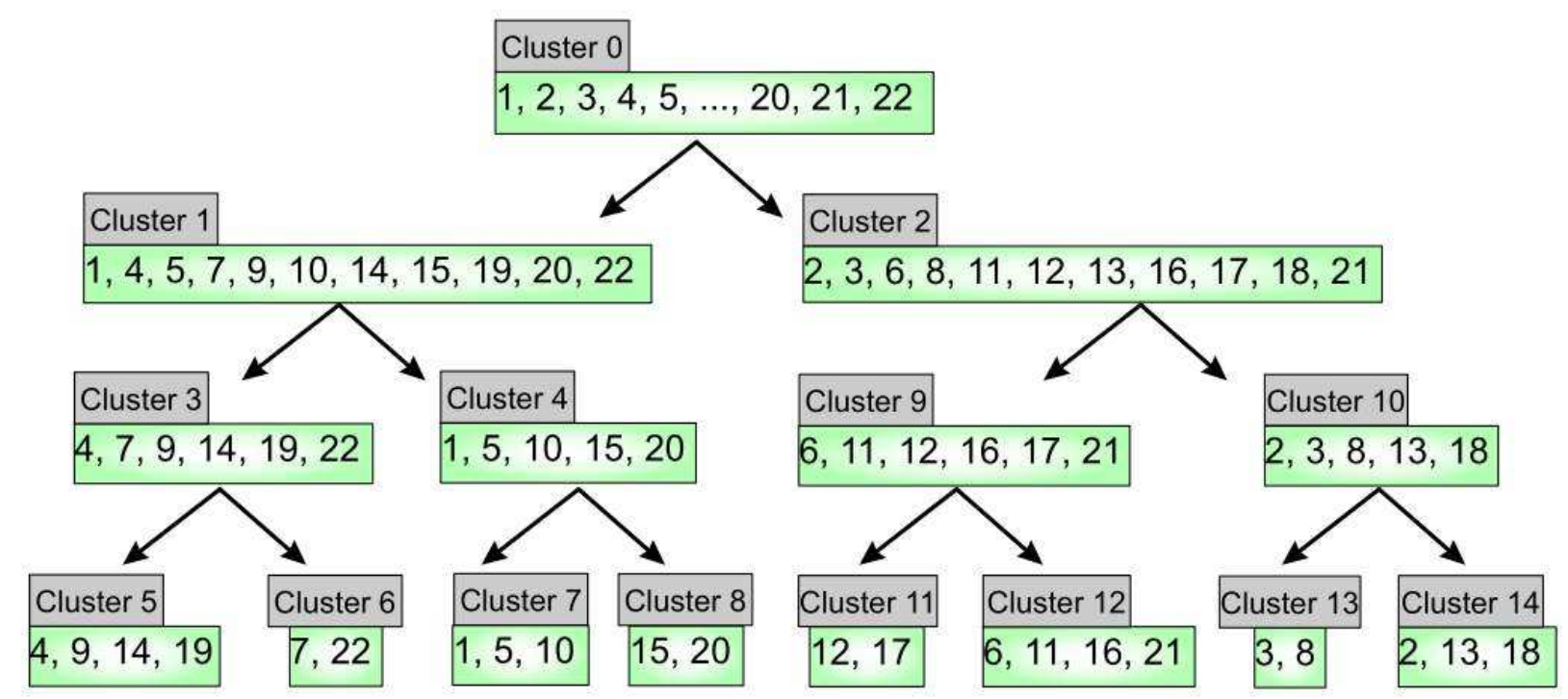

Figure 5.6: Cluster division in a tree format, better known as cluster tree.

As a result of the cluster division 14 clusters were created, from which 8 clusters cannot be further divided. This type of clusters are called leaves, as they resemble the leaves of the cluster tree. However, this information is not sufficient to apply ACA. the ACA is applied to blocks and not to clusters. The block division was implemented following (KURZ et al., 2007); there exist several other good references in the literature about block construction, see, e.g. (BEBENDORF AND RJASANOw, 2003), (GRASEDYCK, 2005), (BENEDETTI et al., 2008), (BRANCATI et al., 2009), (BENEDETTI et al., 2011). 

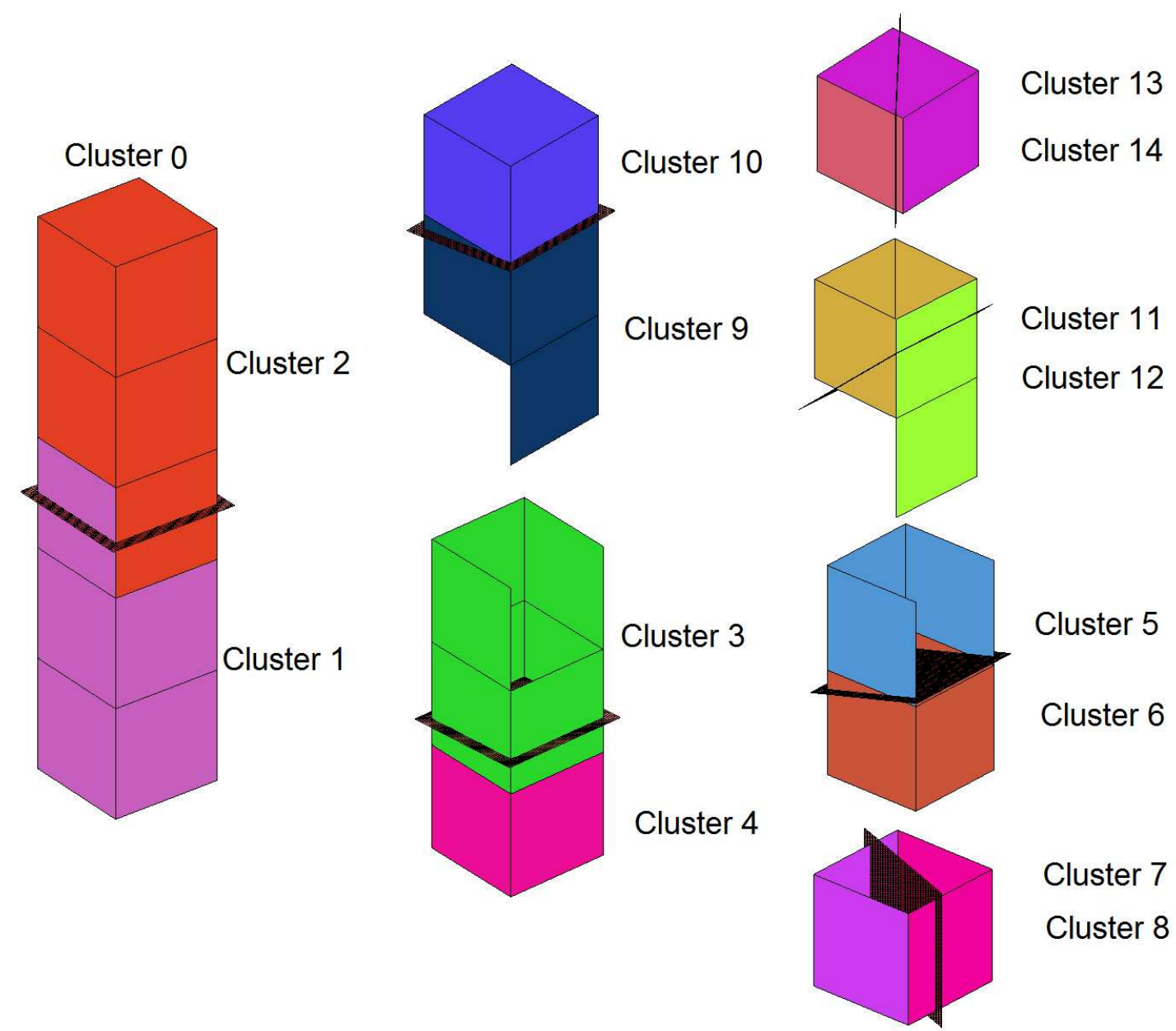

Figure 5.7: Cluster division - geometrical display.

The block division is also done recursively and the objective is to split the collocation matrix hierarchically into sub-blocks and further classify the sub-blocks into admissible or nonadmissible ones. In this process every block is divided into four sub-blocks until either the admissibility condition is satisfied or the block is small enough that it cannot be subdivided. It is worth to point out that every block is formed by two clusters and normally every cluster is formed by 2 sons. Figure 5.8 shows how the block generation is done. Blocks in green are blocks that cannot be divided since the clusters from where they are constructed are leaves. Blocks in yellow are admissible blocks. 


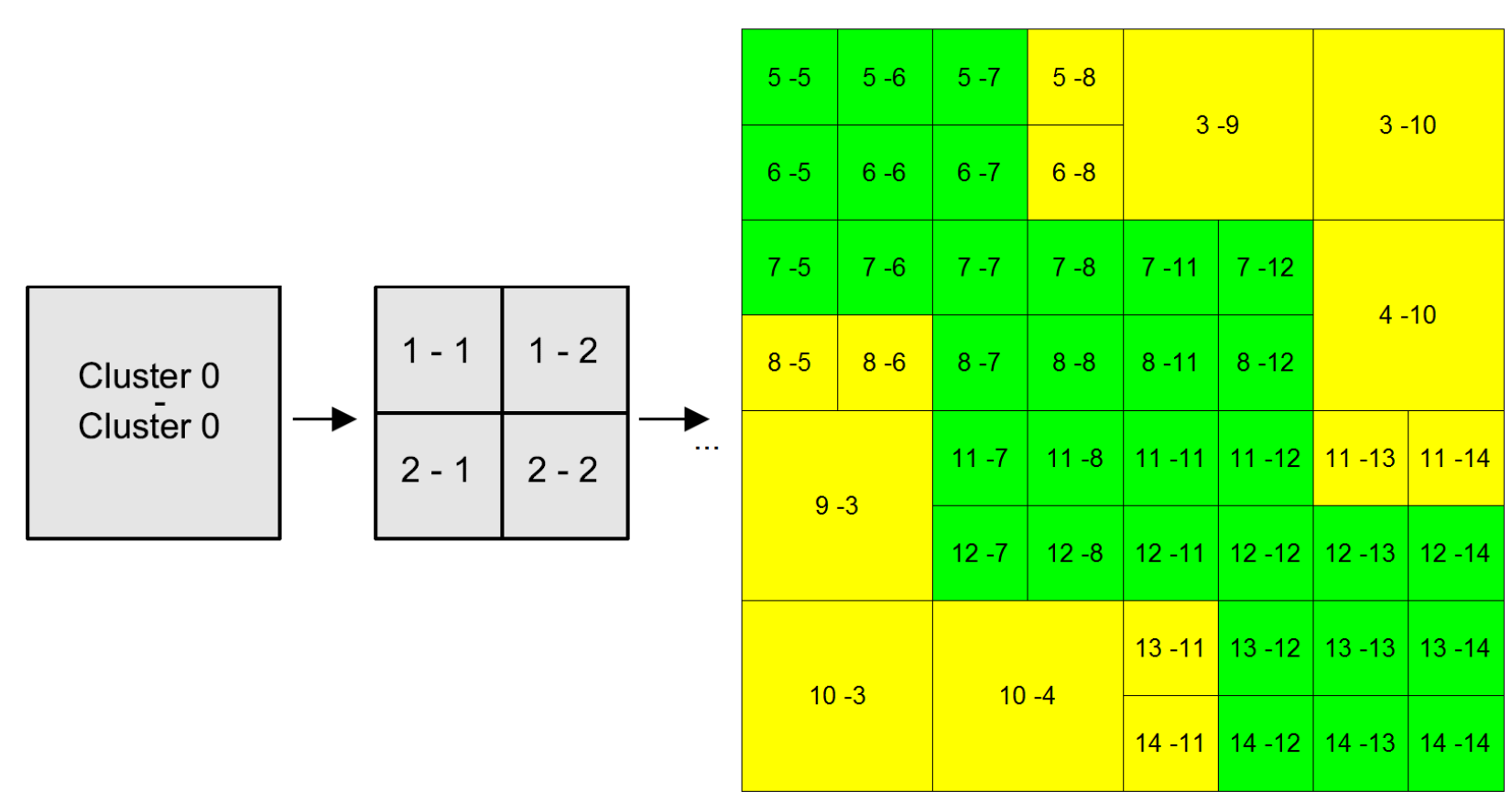

Figure 5.8: Block generation.

Finally, blocks that are admissible (yellow) allow the application of the Adaptive Cross Approximation.

\subsubsection{Example B: ACA applied to a multiple inclusion problem in 2D potential theory}

To test the efficiency of ACA in terms of the solution time and accuracy of the homogenization method described in Section 3.5, two configurations, as shown in Figure 5.9, are analyzed. In the first part, ACA is applied to a regular lattice composite. Six cases with different inclusion configurations are analyzed: 14x14, 18x18, 21×21, 24x24, 26x26 and 28x28 inclusions are modeled in a $1 \times 1$ plate. The plate is modeled with $2 \mathrm{D}$ constant elements. The lower and upper sides are prescribed zero flux condition, and the left and right sides have zero and one degree, respectively. An illustration with 10x10 inclusions is shown in Figure 5.9 (a), with the conditions considered. The fiber volume fraction $c$ is taken to be $12.57 \%$, the matrix conductivity $k_{m}$ is set to 1 and the fibre conductivity $k_{f}$ is set to 0.1 . 


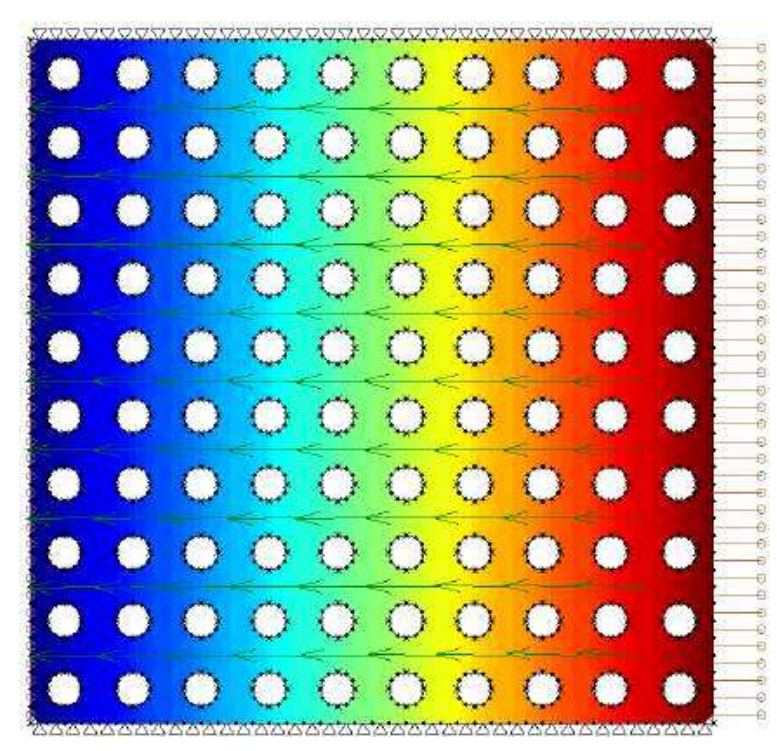

(a)

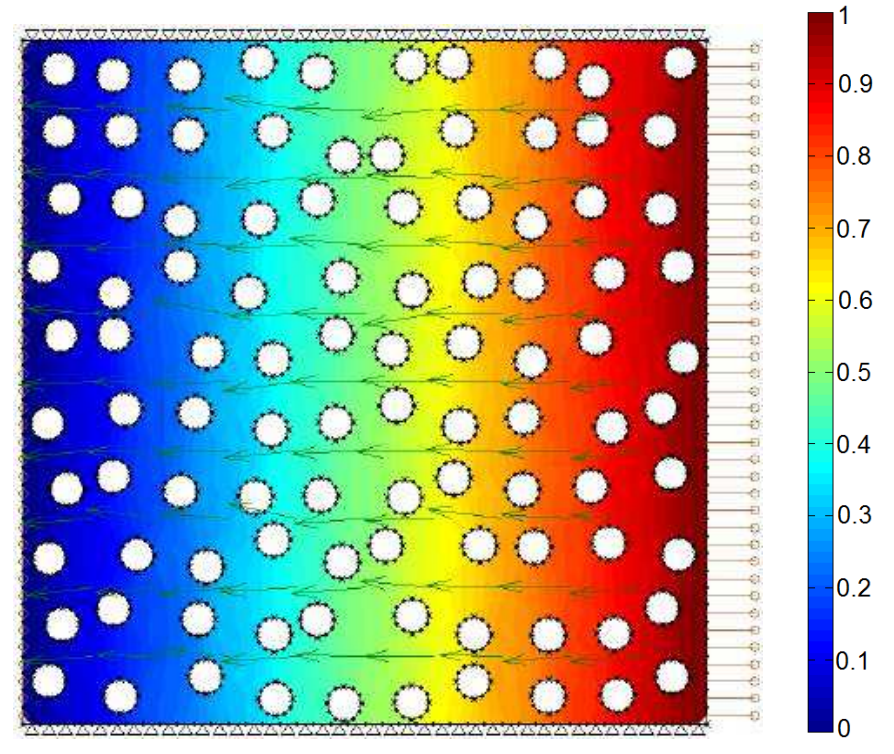

(b)

Figure 5.9: Illustrative figure showing the regular lattice (a), and the shaking-geometry configuration (b).

Figure 5.10 shows the block-wise representation of the collocation matrix for $\eta=0.6,0.8$, 1.0, $\sqrt{2.0}$ and 2.0. Every block is colored showing the ratio between the memory required for low rank representation and the memory in full rank format. The red color (ratio $=1$ ), means that the block was generated as full rank. A helpful colorbar is also shown in Figure 5.9. Table 5.1 shows CPU time ratios and the quantity of blocks generated by the hierarchical method for each case.

It is worth noting that due to the sparseness of the matrices generated by the multidomain method the solution of the system has been obtained using the solver PARDISO, included in the Intel Math Kernel Library (MKL). The selected ACA relative error for all cases was $10^{-5}$. A node by node check of the temperature and flux solution confirmed, that, for a selected accuracy, the average differences are within $\pm 0.1 \%$. Bigger percentage differences can occur for degrees of freedom whose standard solution values are smaller than the requested relative error. This consideration suggests that it is advisable to set the accuracy at the same order of magnitude as that of the smaller quantities of interest in the analysis (BENEDETTI et al., 2011). 
Table 5.1: CPU time ratios for each case

\begin{tabular}{|c|c|c|c|c|c|}
\hline Case & $\eta$ & Blocks & $\begin{array}{c}\text { Admiss. } \\
\text { Pairs }\end{array}$ & $\begin{array}{l}\text { CPU time } \\
\text { ratio }\end{array}$ & $\begin{array}{l}\text { Conventional } \\
\text { BEM time (s) }\end{array}$ \\
\hline \multirow{5}{*}{ Case 1 - 3256e } & 0.6 & 11058 & 300 & 2.16 & \multirow{5}{*}{1.89} \\
\hline & 0.8 & 10402 & 410 & 2.28 & \\
\hline & 1.0 & 8424 & 572 & 2.57 & \\
\hline & $\sqrt{2.0}$ & 2334 & 394 & 4.66 & \\
\hline & 2.0 & 368 & 140 & 6.52 & \\
\hline \multirow{5}{*}{ Case $2-5384 \mathrm{e}$} & 0.6 & 11338 & 256 & 1.41 & \multirow{5}{*}{7.63} \\
\hline & 0.8 & 10584 & 460 & 1.45 & \\
\hline & 1.0 & 8746 & 624 & 1.71 & \\
\hline & $\sqrt{2.0}$ & 2406 & 402 & 3.32 & \\
\hline & 2.0 & 378 & 148 & 4.37 & \\
\hline \multirow{5}{*}{ Case 3 - 7336e } & 0.6 & 11778 & 250 & 1.10 & \multirow{5}{*}{18.16} \\
\hline & 0.8 & 10848 & 470 & 1.20 & \\
\hline & 1.0 & 9062 & 658 & 1.41 & \\
\hline & $\sqrt{2.0}$ & 2424 & 402 & 3.04 & \\
\hline & 2.0 & 388 & 148 & 4.81 & \\
\hline \multirow{5}{*}{ Caso 4 - 9536e } & 0.6 & 11248 & 278 & 0.94 & \multirow{5}{*}{39.07} \\
\hline & 0.8 & 10554 & 438 & 1.02 & \\
\hline & 1.0 & 9062 & 658 & 1.41 & \\
\hline & $\sqrt{2.0}$ & 2424 & 402 & 3.04 & \\
\hline & 2.0 & 388 & 148 & 4.81 & \\
\hline \multirow{5}{*}{ Case 5 - 11176e } & 0.6 & 11685 & 196 & 0.84 & \multirow{5}{*}{62.80} \\
\hline & 0.8 & 11047 & 352 & 0.88 & \\
\hline & 1.0 & 8989 & 562 & 1.07 & \\
\hline & $\sqrt{2.0}$ & 2465 & 392 & 2.45 & \\
\hline & 2.0 & 337 & 138 & 3.43 & \\
\hline \multirow{5}{*}{ Caso $6-12944 \mathrm{e}$} & 0.6 & 11902 & 242 & 0.76 & \multirow{5}{*}{95.73} \\
\hline & 0.8 & 10916 & 422 & 0.79 & \\
\hline & 1.0 & 9090 & 610 & 0.97 & \\
\hline & $\sqrt{2.0}$ & 2517 & 406 & 2.15 & \\
\hline & 2.0 & 344 & 136 & 3.13 & \\
\hline
\end{tabular}

Figure 5.11 shows the CPU time ratio as a function of the number of elements, and as a function of the admissibility parameter $\eta$. Note again that the CPU time ratio is less than one 
only beyond a combination of the number of elements and $\eta$. For our numerical example, the method works better for a number of elements greater than 10,000 and just for the cases where $\eta$ is lower or equal to one. 


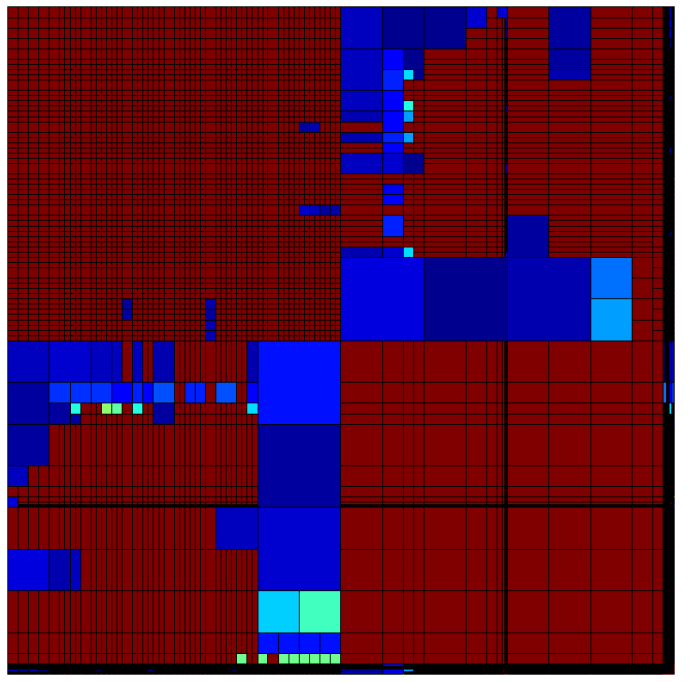

(a)

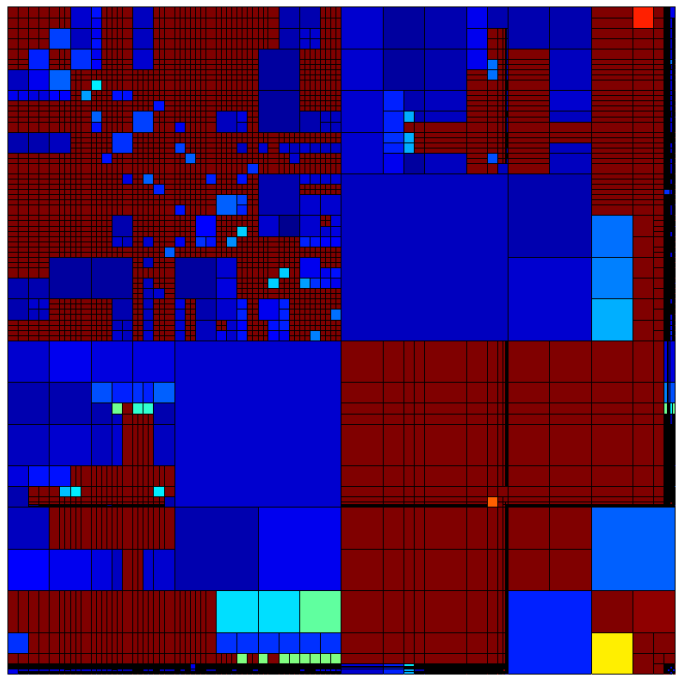

(c)

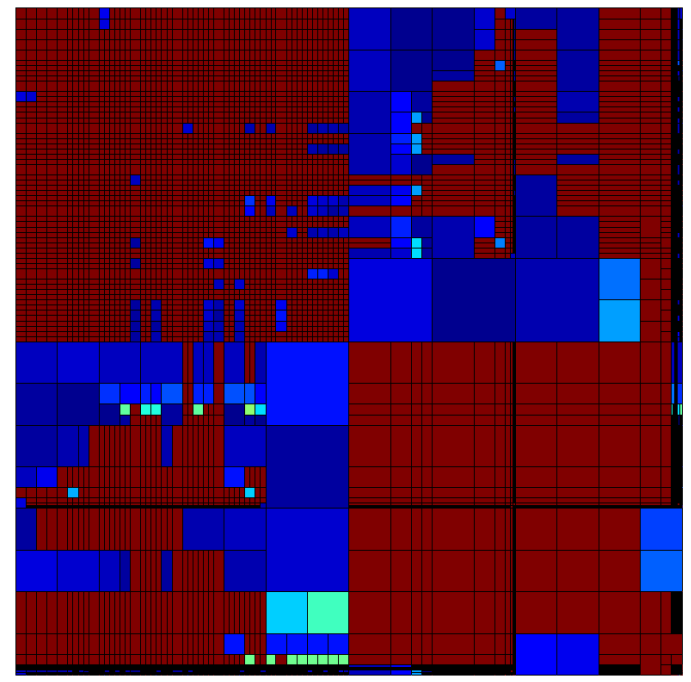

(b)

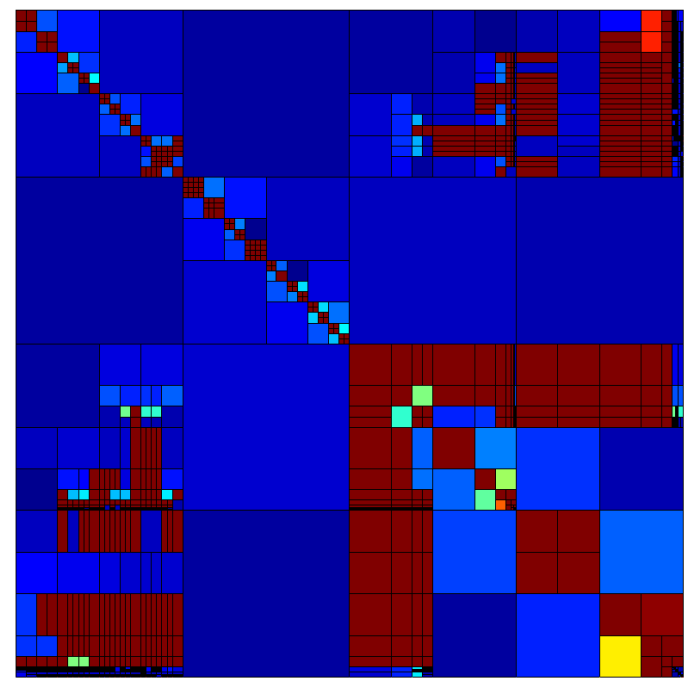

(d)

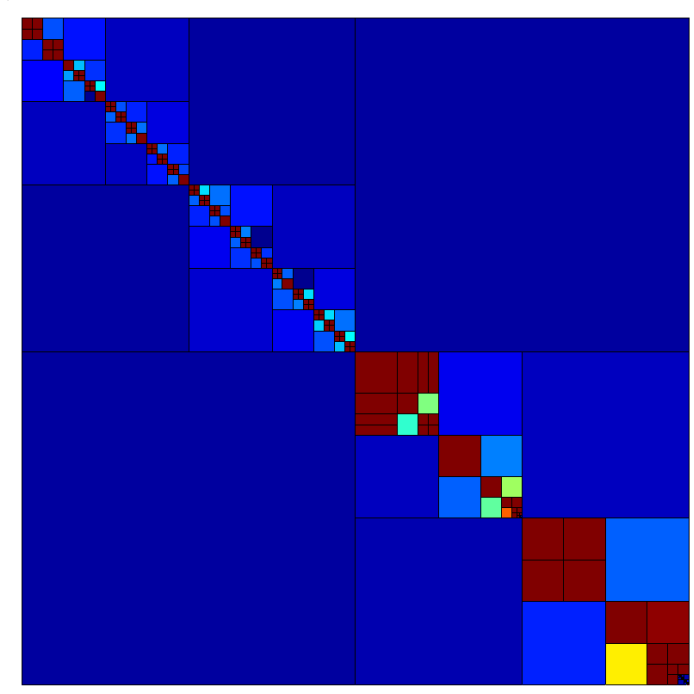

(e)

Figure 5.10: Block-wise representation of the collocation matrix for different admissibility parameters ( $\eta$ ): (a) $\eta=0.6$, (b) $\eta=0.8$, (c) $\eta=1.0$, (d) $\eta=\sqrt{2.0}$ and (e) $\eta=2.0$. 


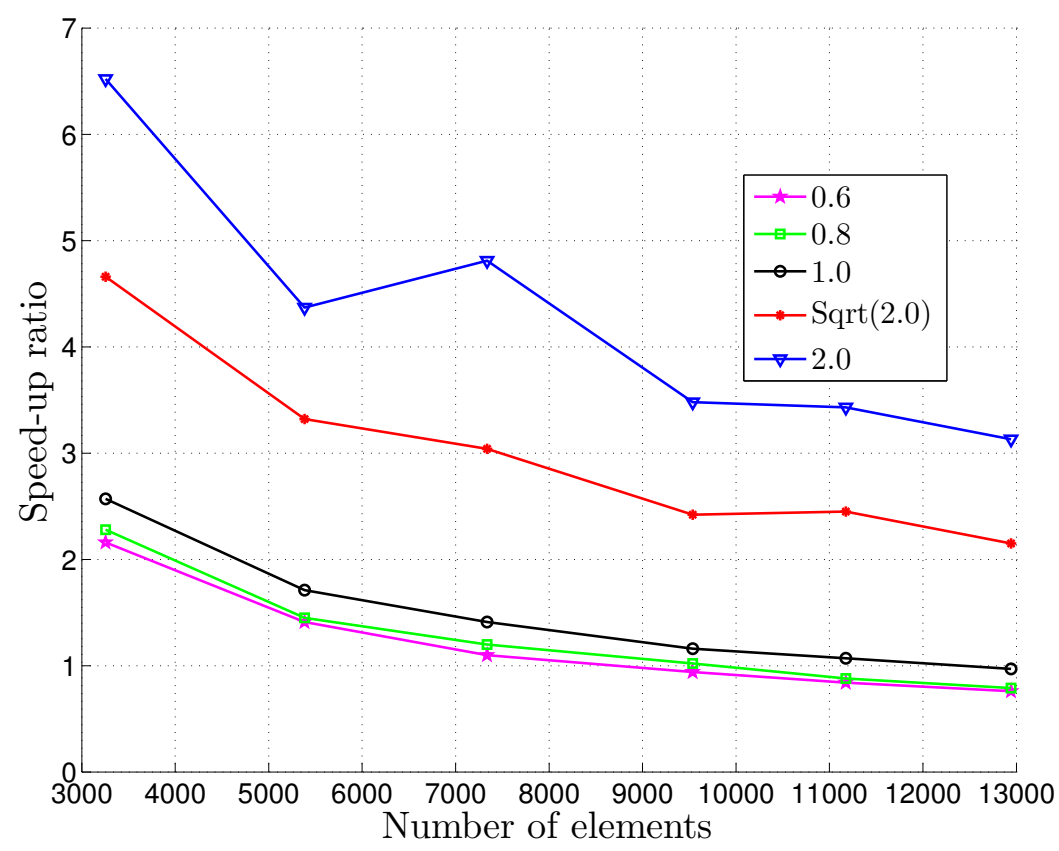

(a)

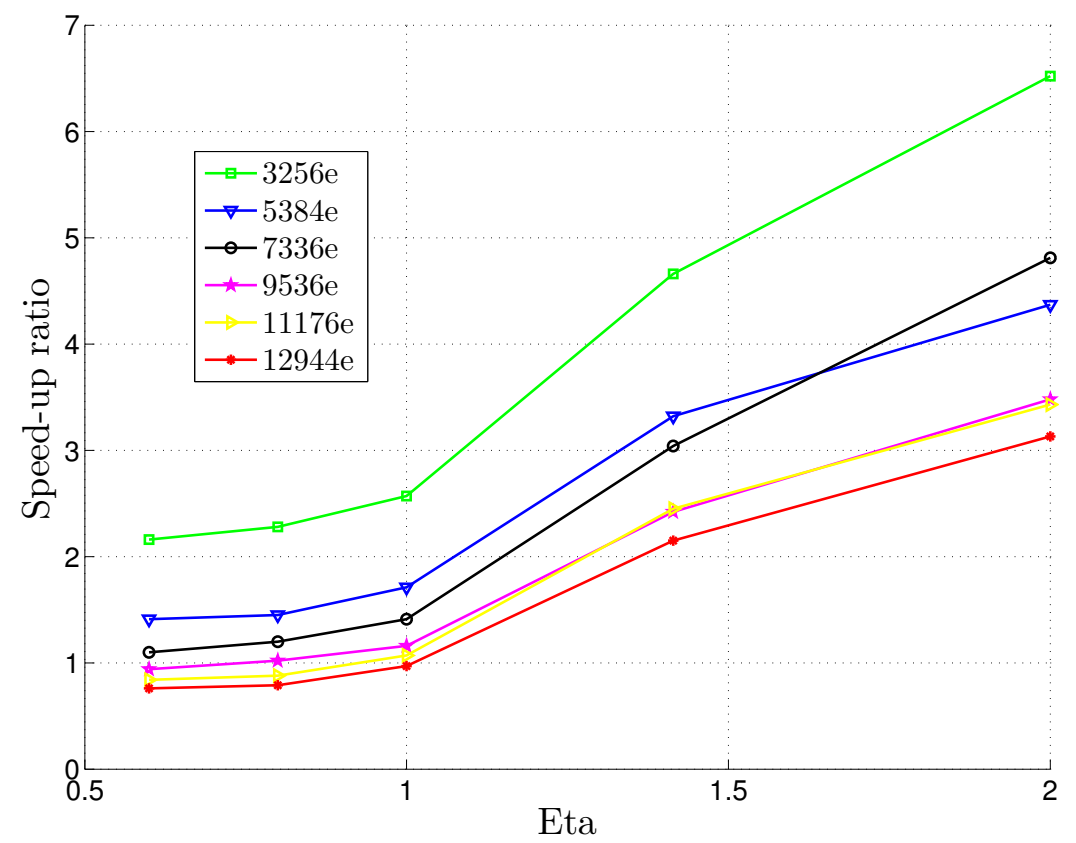

(b)

Figure 5.11: (a) Speed-up ratio as a function of the number of elements, (b) Speed-up ratio as a function of $\eta$.

Finally, the effective conductivity of the plate containing the shaking-geometry, Figure 5.9 (b), is analyzed by using the ACA. The effective conductivity shown in Table 5.2 is calculated 
applying Fourier law to the entire plate. Results are compared with the analytical formulation presented in Section 3.5. Table 5.2 lists the comparison between the implemented ACA model for random composites and the analytical formulation.

Table 5.2: Computed effective conductivity for each case

\begin{tabular}{ccccc}
\hline Case & $\begin{array}{c}\text { No of } \\
\text { Inclusions }\end{array}$ & Total DOFs & $\begin{array}{c}\text { No of } \\
\text { Elements }\end{array}$ & $\begin{array}{c}\text { Effective } \\
\text { Conductivity }\end{array}$ \\
\hline 1 & $14 \times 14$ & 6392 & 3256 & 0.8152 \\
2 & $18 \times 18$ & 10568 & 5384 & 0.8151 \\
3 & $21 \times 21$ & 14392 & 7336 & 0.8150 \\
4 & $24 \times 24$ & 18752 & 9536 & 0.8148 \\
5 & $26 \times 26$ & 21992 & 11176 & 0.8150 \\
6 & $28 \times 28$ & 25488 & 12944 & 0.8150 \\
\multicolumn{5}{l}{ Analytical estimates, Equation (2.38): } \\
\hline
\end{tabular}

\subsubsection{Example C: ACA applied to an anisotropic elastostatics problem}

The physical problem considered is the same as that shown in Chapter 3, Section 3.6.3, a cube with a cylindrical hole. However, the main objective here is to verify the ACA scheme. As mentioned before, the cube has a hole with a radius of $r=0.3 H$, where $H$ is the length of its side, which are subjected to external pressure $P$, as shown in Figure 3.8(a). The bottom and top surfaces are totally constrained in the three coordinate directions. The material considered is an alpha-quartz with the following stiffness matrix,

$$
\mathbf{C}=\left[\begin{array}{cccccc}
87.6 & 6.07 & 13.3 & 17.3 & 0 & 0 \\
6.07 & 87.6 & 13.3 & -17.3 & 0 & 0 \\
13.3 & 13.3 & 106.8 & 0 & 0 & 0 \\
17.3 & -17.3 & 0 & 57.2 & 0 & 0 \\
0 & 0 & 0 & 0 & 57.2 & 17.3 \\
0 & 0 & 0 & 0 & 17.3 & 40.765
\end{array}\right] \text { GPa }
$$

Four different meshes (192, 384, 704 and 1040 quadratic quadrilateral elements) were analyzed. Figure 5.12(a) shows the coarsest mesh, while Fig. 5.12(b) shows the most refined one employed. 


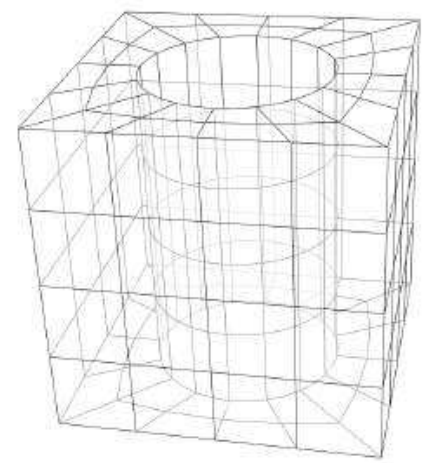

(a)

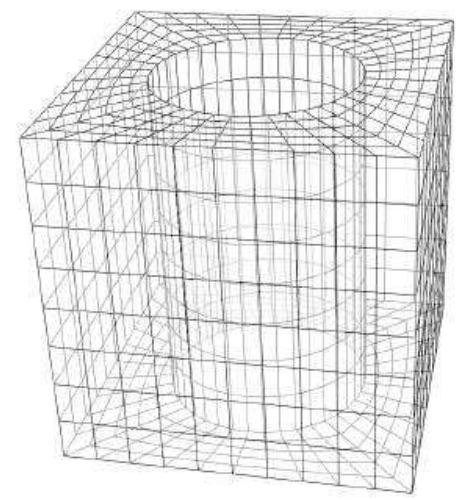

(b)

Figure 5.12: (a) BEM coarsest mesh; (b) BEM most refined mesh.

The ACA error tolerance is set to $\epsilon_{c}=10^{-4}$. Moreover, a SVD recompression is invoked to create a pre-conditioner matrix for the iterative solver, the generalized minimum residual method (GMRES). The recompression tolerance is set to $\epsilon_{c}=10^{-2}$. The maximum number of elements per cluster was set to 40 and the admissibility parameter $(\eta)$ to 0.8 . More details of the choice of these parameters are available in (RODRÍGUEZ et al.; RODRÍGUEZ et al.; BENEDETTI et al., 2012; 2013; 2009). For the most refined case (1040 elements) there were 95 clusters and 2304 blocks created, from which 872 were admissible pairs. Results from the BEM anisotropic formulation using the ACA (for the most refined mesh) were already compared with the FEM results in the Chapter 3, Example C, Figure (3.8). The computer cpu times are compared and are shown in Fig. 5.13. With the finest mesh, the ACA scheme becomes more efficient computationally in obtaining the same accurate results than the conventional BEM when the number of elements exceed about 600 boundary elements. 


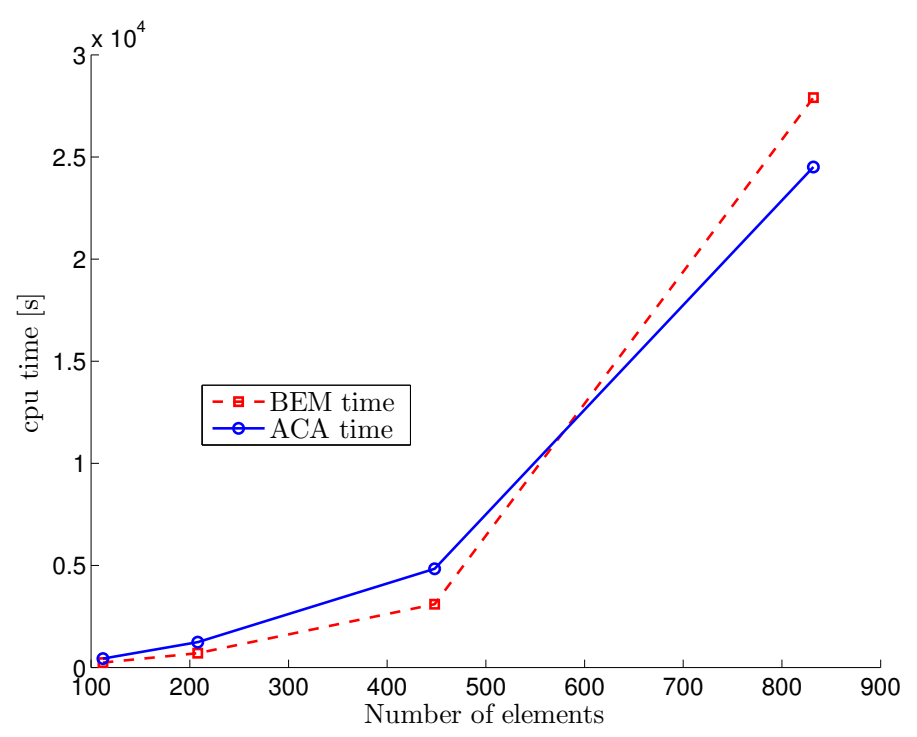

Figure 5.13: BEM and ACA cpu times.

\subsubsection{Example D: ACA applied to another generally anisotropic problem in elas- tostatics}

The physical problem is the same as the shown in Chapter 3, Section 3.6.4., Example D. The main objective is to verify the ACA scheme when highly anisotropic materials are considered. The physical problem is an internally pressurized cylinder made of an alpha-quartz crystal has internal pressure, $P$, with radius ratio $R_{2} / R_{1}=2$ and total length $2 H=8 R_{1}$, as shown in Figure 3.10 (a). The external circumferential surface is constrained in the radial direction, while its two ends are fixed in the $x_{3}$ direction. The principal material axes of the alpha-quartz crystal are successively rotated about the global Cartesian $x_{1}, x_{2}$ and $x_{3}$ axis by $30^{\circ}, 45^{\circ}$ and $60^{\circ}$ clockwise, respectively. These successive rotations yield a fully populated stiffness matrix, as follows,

$$
\mathbf{C}=\left[\begin{array}{cccccc}
111.8 & 14.8 & -5.2 & -0.3 & 11.0 & -14.0 \\
14.8 & 101.8 & -7.6 & 0.4 & -0.6 & 18.9 \\
-5.2 & -7.6 & 129.7 & 4.4 & 1.6 & 0.6 \\
-0.3 & 0.4 & 4.4 & 31.3 & 2.5 & 3.6 \\
11.0 & -0.6 & 1.6 & 2.5 & 37.9 & 1.3 \\
-14.0 & 18.9 & 0.6 & 3.6 & 1.3 & 55.2
\end{array}\right] \mathrm{GPa}
$$


Six different meshes (96, 216, 418, 680, 960 and 1232 quadratic quadrilateral elements) were analyzed. Figure 5.12(b) shows the coarsest mesh, while Fig. 5.14(b) shows the most refined one employed.

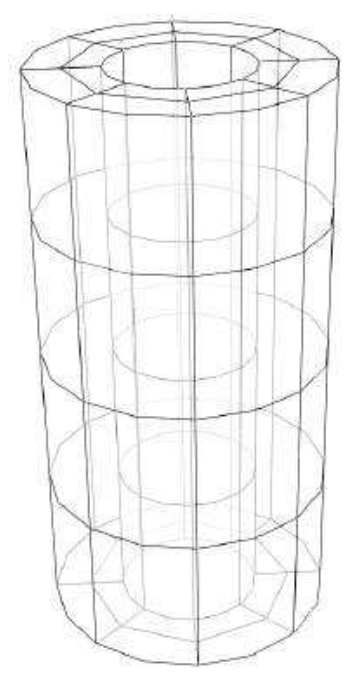

(a)

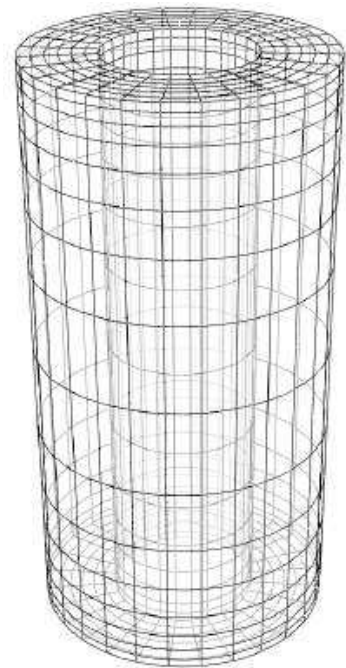

(b)

Figure 5.14: (a) BEM most refined mesh; (b) BEM coarsest mesh.

The ACA error tolerance is set to $\epsilon_{c}=10^{-4}$. The recompression tolerance is set to $\epsilon_{c}=10^{-2}$. The maximum number of elements per cluster was set to 60 and the admissibility parameter $(\eta)$ to 0.8 . For the most refined case (1232 elements) there were 55 clusters and 576 blocks created, from which 96 were admissible pairs. Results from the BEM anisotropic formulation using the ACA (for the most refined mesh) are compared with the FEM results obtained by the commercial software ANSYS, carried out in (TAN et al., 2013). The cpu times are also compared as shown in Fig. 5.15. Again, with high number of elements, the ACA scheme takes less cpu times than the conventional BEM. 


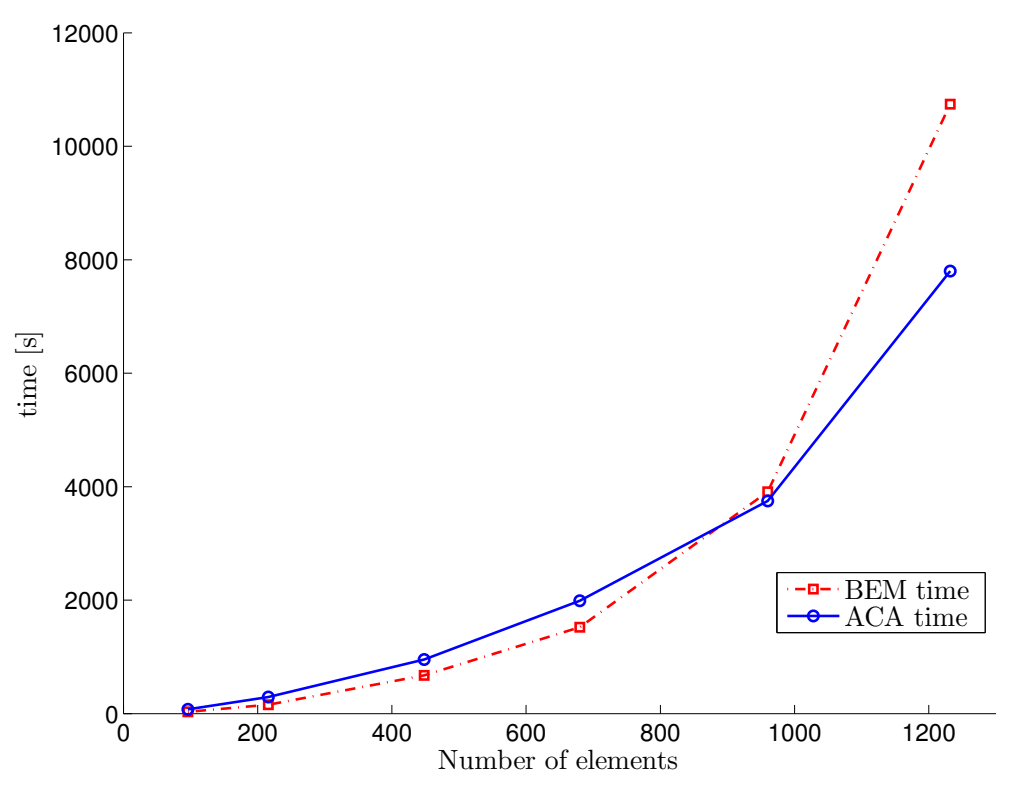

Figure 5.15: Conventional BEM and ACA cpu times.

\subsubsection{Example E: Fiber-matrix model}

In this example, the fiber-matrix model was implemented and tested. A general mesh generator was implemented. The fibers can be randomly oriented, as can be seen in Fig. 5.16. The length and cross-section of the short fibers are also input parameters; thus, the volume fraction can be also imposed. A fiber-matrix model with 36 short fibers is shown in Fig. 5.16(a), while a unit cell is shown in Fig. 5.16(b). 


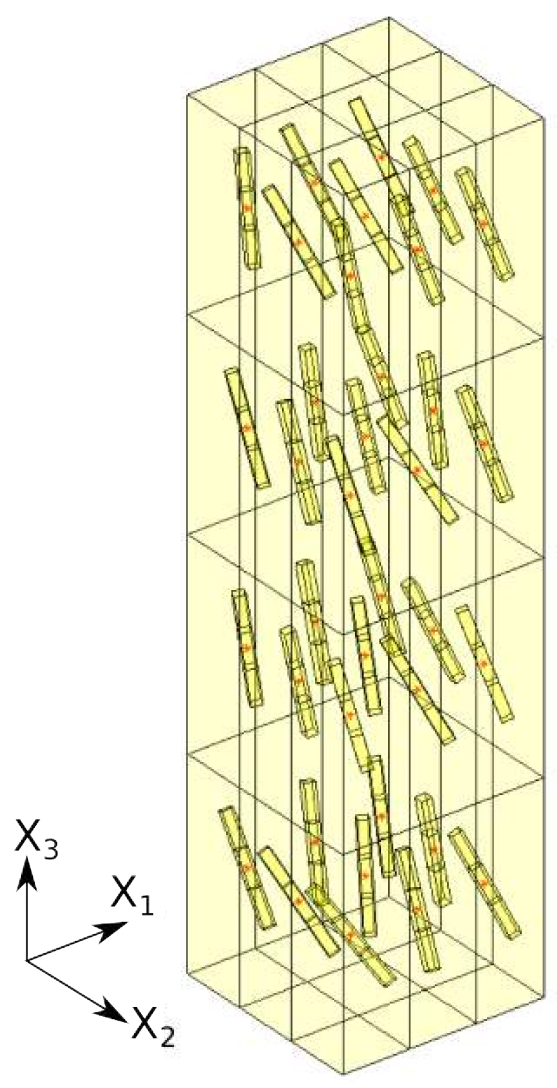

(a)

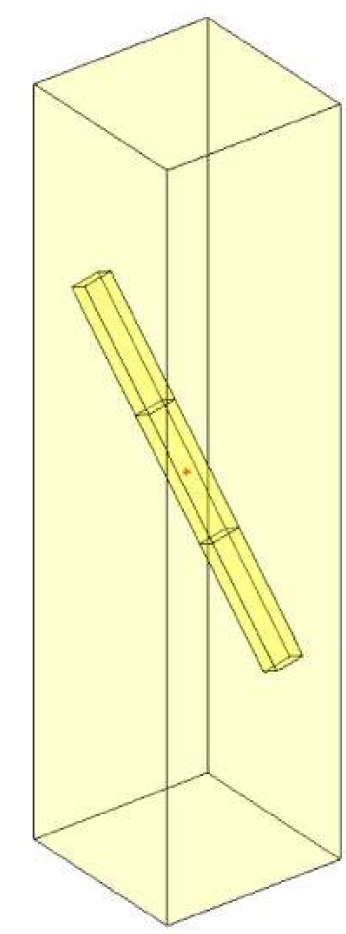

(b)

Figure 5.16: (a) Fiber-Matrix model with 36 fibers; (b) unit cell with a randomly oriented short fiber.

The model was tested with an isotropic material with $E=10000$ and $\nu=0.3$ for the short fibers and the matrix medium, however, the anisotropic 3D fundamental solution based on Fourier series was used. As usual, $\alpha=16$ and 64 Gauss integration points were used for the Fourier representation. An isotropic material was used in order to properly compare results with the analytical solution. For this first fiber-matrix example, a bar of length 4 units and square cross-section of unity side length is subjected to a uniformly distributed pressure load, $P=1$, on its top surface, while, its bottom surface is fully constrained in the three coordinate directions.

The well known analytical isotropic solution for the $u_{3}$ displacement is $P x_{3} / E$. Displacements along the $x_{3}$ direction for the fiber-matrix model are shown in Fig. 5.17. 


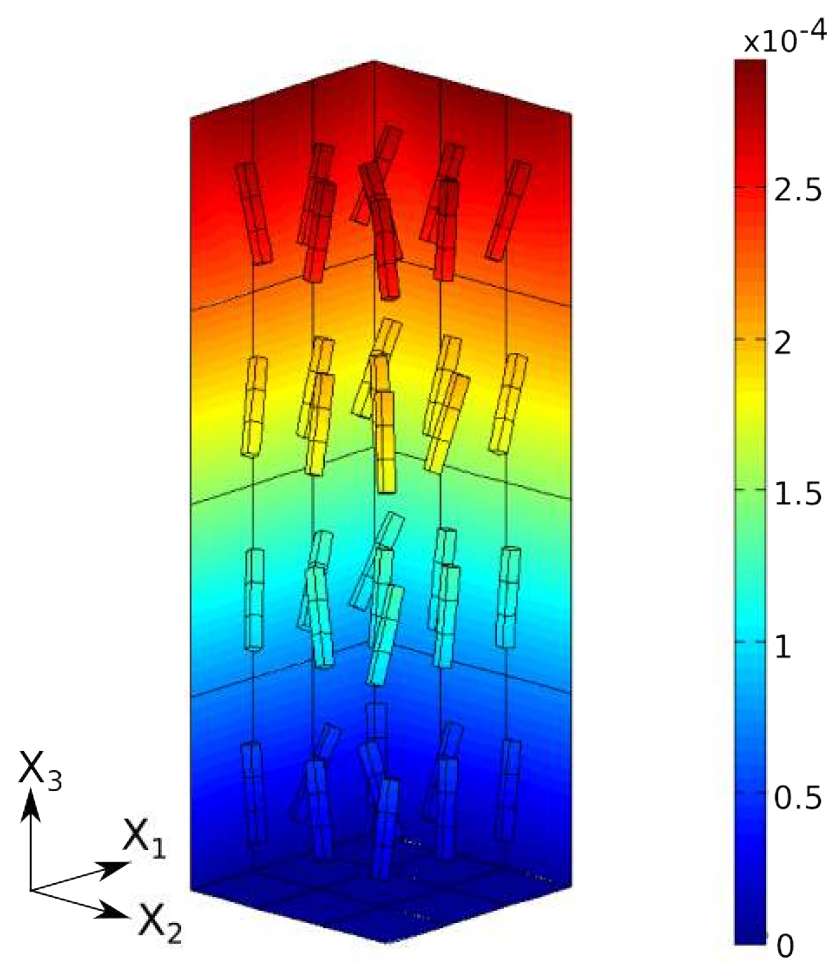

Figure 5.17: Fiber-matrix model with an isotropic material.

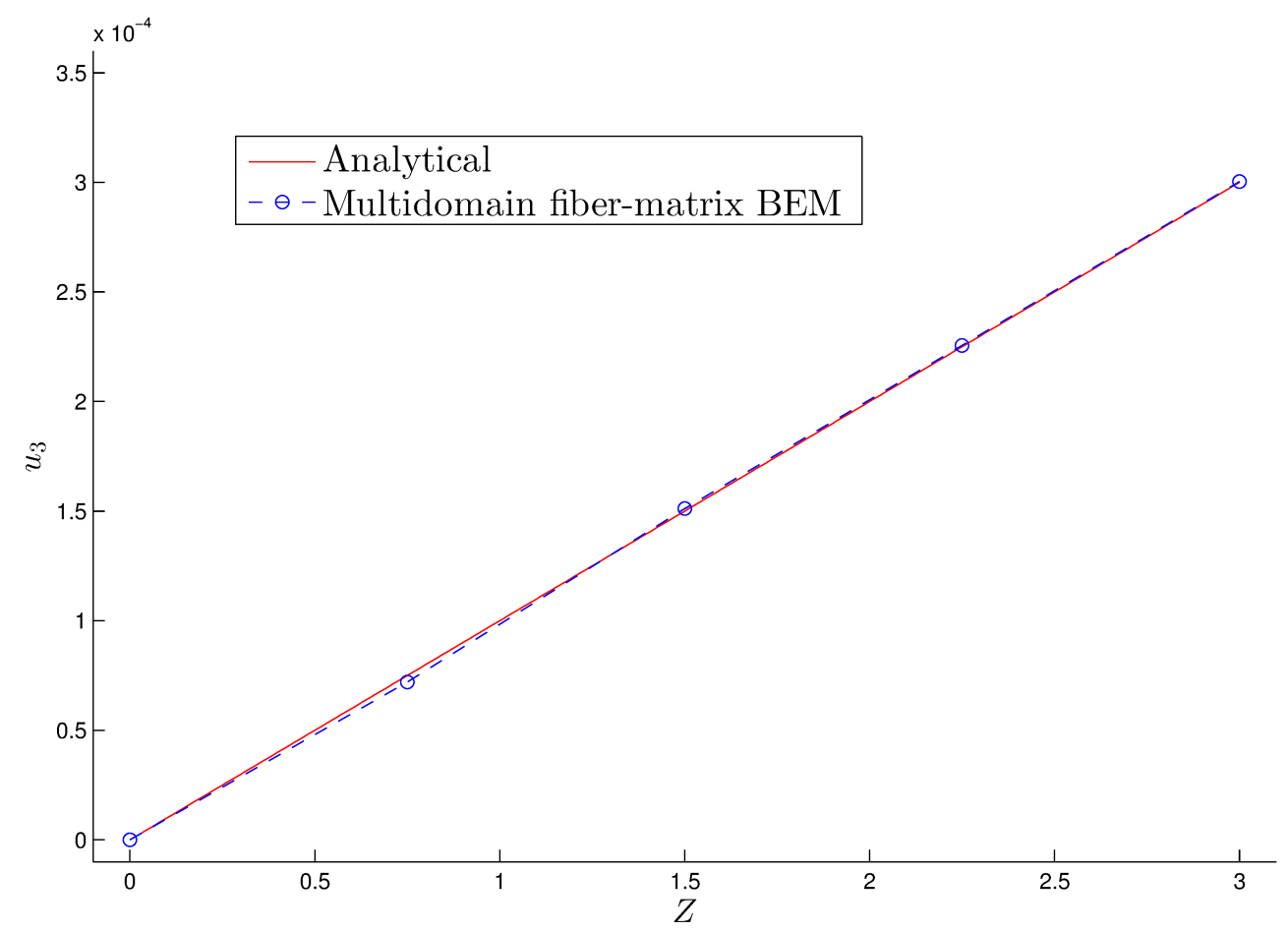

Figure 5.18: Numerical comparison with the analytical solution. 
Displacements at nodes $\left(0.5,0.5, x_{3}\right) \mathrm{u}$ are compared with the analytical solution, as shown in Fig. 5.18. Good agreement is observed when the results of the fiber-matrix multidomain model was compared with the analytical solution.

Next, the fiber-matrix model was tested with a more realistic combination of materials for both, fiber and matrix. Furthermore, the ACA method was applied in the generation of BEM matrices $[\mathrm{H}]$ and $[\mathrm{G}]$. For the fiber, a 3501-6 epoxy was considered. Material properties for the fiber were considered as isotropic with $E=4.2 \mathrm{GPa}$ and Poisson ratio $\nu=0.34$. For the matrix, a AS4 carbon fiber was considered. Material properties for the matrix were considered as transversely isotropic with $E_{1}=225 \mathrm{GPa}, E_{2}=15 \mathrm{GPa}, G_{12}=15 \mathrm{GPa}$ and $\nu_{12}=0.2$. More details on the properties of the chosen combination fiber-matrix can be seen in (SODEN et al., 1998).

In this example, a bar of length 3 units and square cross-section of side length unity was subjected to a uniformly distributed pressure load, $P=1$, on its top surface, while, its bottom surface is restricted in the 3 coordinate directions. In order to test the ACA method, five different cases were analysed $\left(4,8,12,18\right.$ and 27 fibers). The ACA error tolerance is set to $\epsilon_{c}=10^{-4}$. The maximum number of elements per cluster was set to 30 and the admissibility parameter, $\eta$, to 0.9. More details of the choice of these parameters are available in (BENEDETTI et al.; RODRÍGUEZ et al.; RODRÍGUEZ et al., 2009; 2012; 2013). For the most refined case (27 fibers, 6480 quadratic discontinuous nodes) there were 45 clusters and 487 blocks, from which 46 were admissible pairs. Results from the BEM anisotropic multidomain model using the ACA for the $u_{3}$ displacement are shown in Figure (5.19). 


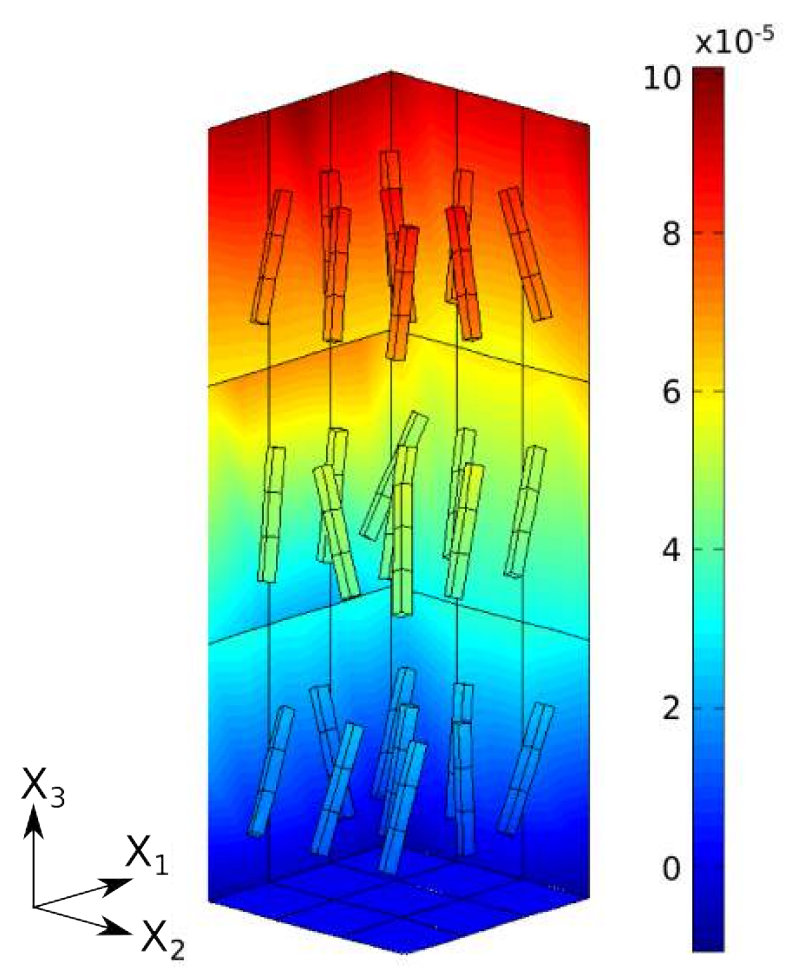

Figure 5.19: Displacement on the $x_{3}$ direction for the most refined case.

Table 5.4 shows comparison of the computed displacements $u_{1}, u_{2}$ and $u_{3}$ between conventional BEM and ACA. Five random nodes (349, 841, 1050, 3439 and 6052) were chosen for the comparison, as can be seen in Table 5.3. The percentage discrepancies of the numerical results are also as indicated in the Table 5.4.

Table 5.3: Coordinates of the random nodes.

\begin{tabular}{cc}
\hline Node & Coordinates $(x, y, z)$ \\
\hline 349 & $(0.000,0.176,1.050)$ \\
841 & $(0.163,0.528,0.577)$ \\
1050 & $(0.209,0.509,2.412)$ \\
3439 & $(0.791,0.817,2.583)$ \\
6052 & $(0.715,0.585,2.696)$ \\
\hline
\end{tabular}


Table 5.4: Nodal comparison between conventional BEM and ACA for random nodes.

\begin{tabular}{ccccccc}
\hline & BEM & ACA & BEM & ACA & BEM & ACA \\
\hline Node & $u_{1} x 10^{-6}$ & $u_{1} x 10^{-6}($ Err.\%) & $u_{2} x 10^{-6}$ & $u_{2} x 10^{-6}($ Err.\%) & $u_{3} x 10^{-4}$ & $u_{3} x 10^{-4}$ (Err.\%) \\
\hline 349 & -0.4341 & $-0.4325(0.37)$ & 26.750 & $26.790(0.15)$ & 0.2916 & $0.2912(0.14)$ \\
841 & 1.5440 & $1.5470(0.19)$ & -0.8381 & $-0.8246(1.61)$ & 0.1564 & $0.1564(0.00)$ \\
1050 & 1.1260 & $1.1270(0.09)$ & -0.2478 & $-0.2321(6.34)$ & 0.7561 & $0.7562(0.01)$ \\
3439 & -0.2270 & $-0.2335(2.86)$ & -5.1020 & $-5.0660(0.71)$ & 0.7826 & $0.7829(0.04)$ \\
6052 & -0.5833 & $-0.5849(0.27)$ & -0.5269 & $-0.5118(2.87)$ & 0.8506 & $0.8508(0.02)$ \\
\hline
\end{tabular}

The maximum discrepancy was $6.34 \%$ for the displacement in the $x_{2}$ direction at node 1050. For this case, the displacement was $-0.2478 \times 10^{-6} \mathrm{~mm}$ for the conventional BEM, being substantially less than the ACA tolerance of $\epsilon_{c}=10^{-4}$. As pointed out by (BENEDETTI et al., 2011), the ACA tolerance should be set to be compatible with the expected smallest response value. Figure (5.20) shows the comparison between cpu times for generating BEM matrices for conventional BEM and when the ACA method is applied. It can be noted that when the numerical problem size gets to be large, the ACA scheme becomes significantly more efficient than the conventional BEM.

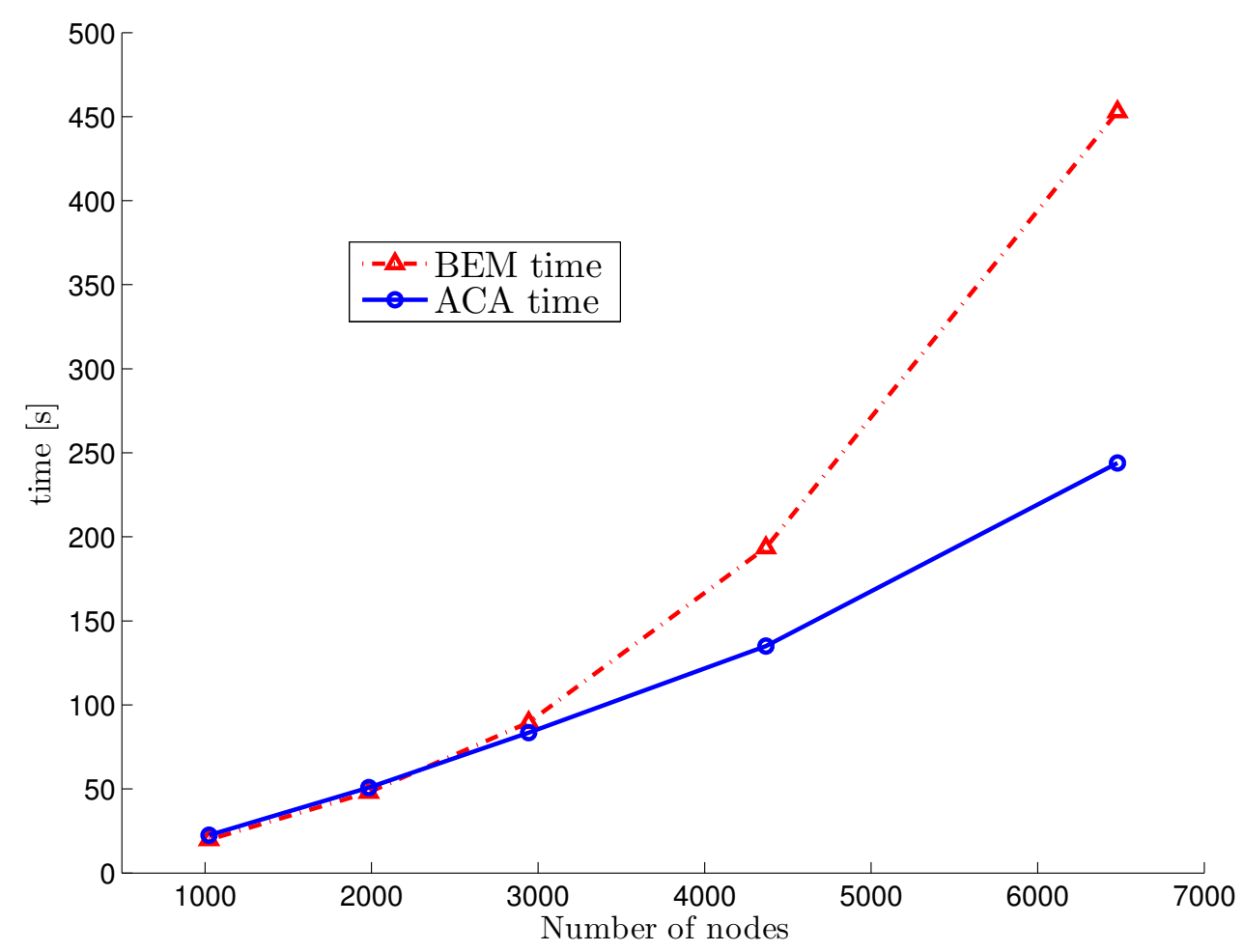

Figure 5.20: Cpu times comparison between conventional BEM and ACA. 


\section{Multi-scale failure analysis of laminated composites}

\subsection{Introduction}

As a final objective of the present study, a synthesis of all the work developed in the previous chapters is carried out with an appropriate failure criterion to demonstrate the use of the BEM for multi-scale failure analysis of composites. But first, the cohesive zone atomisticmolecular potential is reviewed. The potential considered is for the epoxy materials, and it is a combination of repulsive Lennard-Jones interactions and a quartic bond potential, adapted from Yang et al. (2014). The means to obtain numerically the deformation gradient in the cohesive zone, as well as, applying the Cauchy-Born rule is also described in this chapter. Then, the cohesive law that determines the failure criterion is presented. Finally, some numerical examples are presented to demonstrate the failure criterion to laminated composites. It is worth to point out that the failure criterion is based in a multi-scale coupling approach and not in fracture mechanics. Classical fracture mechanics parameters such as the stress intensity factor or the $\mathrm{J}$ integral are not required for the failure propagation, which is accounted by the removal of cohesive zones.

\subsection{Cohesive zone atomistic-molecular potential}

The cohesive strength in the cohesive zone of a continuum at the microscopic level can be evaluated by integrating all the intermolecular forces surrounding the bulk medium. For convenience, the bulk medium is considered to be rigid, so that the interaction between two material points inside the cohesive zone and the bulk medium can be lastly calculated. The development of a proper multiscale cohesive law should be based on the material physical decohesion such as atomistic and molecular interactions. These interactions could be physically represented by potentials. The embedded atom method (EAM) potential provides an accurate description of interatomic reactions considering the effects of electron density distribution, (DAW AND BASKES, 1983), (DAW AND BASKES, 1984). Nonetheless, it is highly dependent on the lattice structure and the availability of experimental data. Several EAM potentials are available in the literature, e.g., for face centered cubic (FCC) (YUAN et al., 2010), (OH AND JOHNSON, 1992), body centered cubic (BCC) (JOHNSON AND OH, 1989), and hexagonal close packed (HCP) (OH AND JOHNSON, 1992) materials. For these cases a simple pair-wise potential, namely the Lennard-Jones (LJ) potential, Equation (6.1), is generally used in cohesive zone models, 


$$
\phi_{\text {bulk }}=4 \epsilon\left[\left(\frac{\sigma}{r}\right)^{12}-\left(\frac{\sigma}{r}\right)^{6}\right]
$$

where $\epsilon$ is the depth of the potential well, $\sigma$ is the finite distance when the bulk atomistic potential is equivalent to zero.

Then, the interface potential, $\phi_{\text {int }}$, can be obtained by analytical integration, (IsRAelaCHVILI, 1992), (ZENG AND Li, 2012), (GALVIS AND SOllero, 2014).

$$
\phi_{\text {int }}=\frac{\pi \epsilon}{\sqrt{2}}\left[\frac{1}{45}\left(\frac{r_{0}}{r}\right)^{9}-\frac{1}{3}\left(\frac{r_{0}}{r}\right)^{3}\right]
$$

where $r_{0}=2^{1 / 6} \sigma$ is the equilibrium bond distance in the bulk material.

Recently, a coarse-grained model for epoxy compounds was proposed by (YANG et al., 2014). In this model the epoxy and hardener molecules are represented as short chains of connected beads. The potential parameters are found through an optimization process in which thermo-mechanical properties of the material obtained by experimental tests are considered. Figure(6.1) shows epoxy compound represented by short chains of beads.
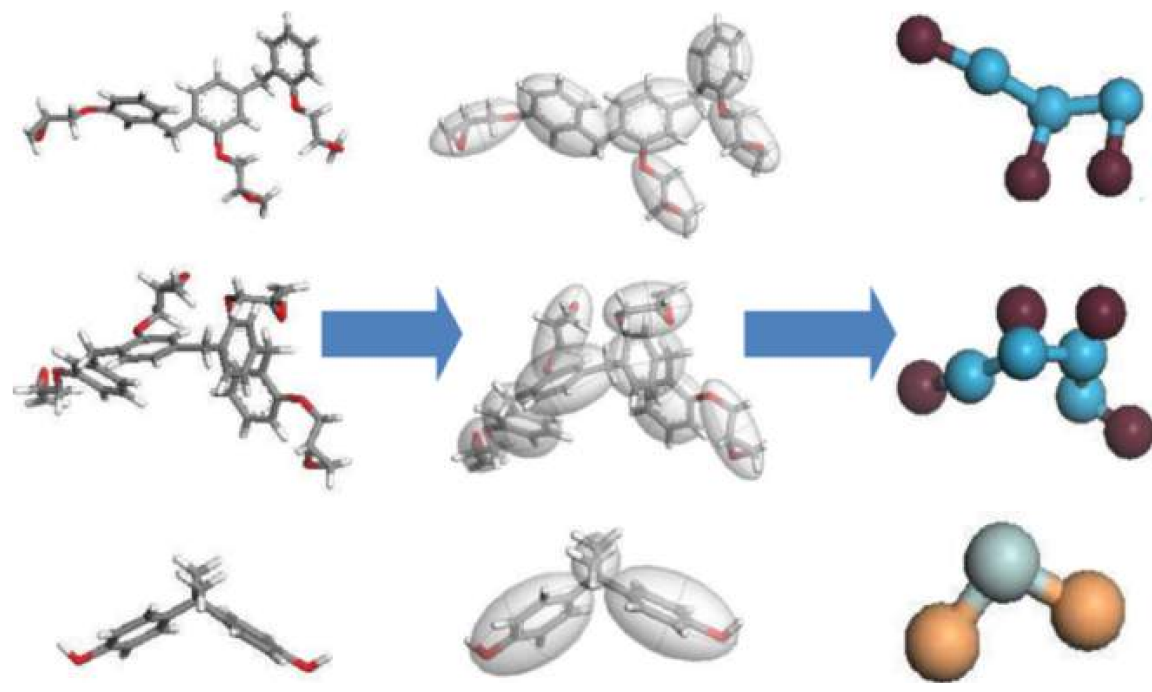

Figure 6.1: Epoxy compound represented as short chains of beads (YANG et al., 2014).

In the model shown by (YANG et al., 2014), the bonds between beads are described by a potential that considers bond breakage. The potential, $\phi_{g}$, is a sum of purely repulsive Lennard- 
Jones interactions, with a cut-off at $2^{1 / 6} \sigma$, and a quartic bond potential (LORENZ et al., 2004),

$$
\begin{aligned}
\phi_{g}(r)= & U_{0}+k\left(r-r_{c}\right)\left(r-b_{1}-r_{c}\right)\left(r-b_{2}-r_{c}\right) H\left(r_{c}-r\right)+ \\
& 4 \epsilon\left[\left(\frac{\sigma}{r}\right)^{12}-\left(\frac{\sigma}{r}\right)^{6}+\frac{1}{4}\right] H\left(2^{1 / 6} \sigma-r\right) H\left(r_{c}-r\right)
\end{aligned}
$$

where $H(x)$ is the Heaviside step function, $k=1434.3 \epsilon / \sigma^{4}, b_{1}=-0.7589 \sigma, b_{2}=0$ and $U_{0}=67.2234 \epsilon$. Figure (6.2) shows the effect of the cut-off, $r_{c}$, on the bond potential. The figure indicates that the equilibrium distance and the potential well depth, $\epsilon$, decreases when $r_{c}$ decreases. Thus, the potential is truncated at $r_{c}$ and this represent an adequate failure criterion for bond breakage. Other parameters could be considered, such as angle bending interaction; however, the study of those parameters is not in the scope of the present work. For more details refer to (LORENZ et al., 2004).

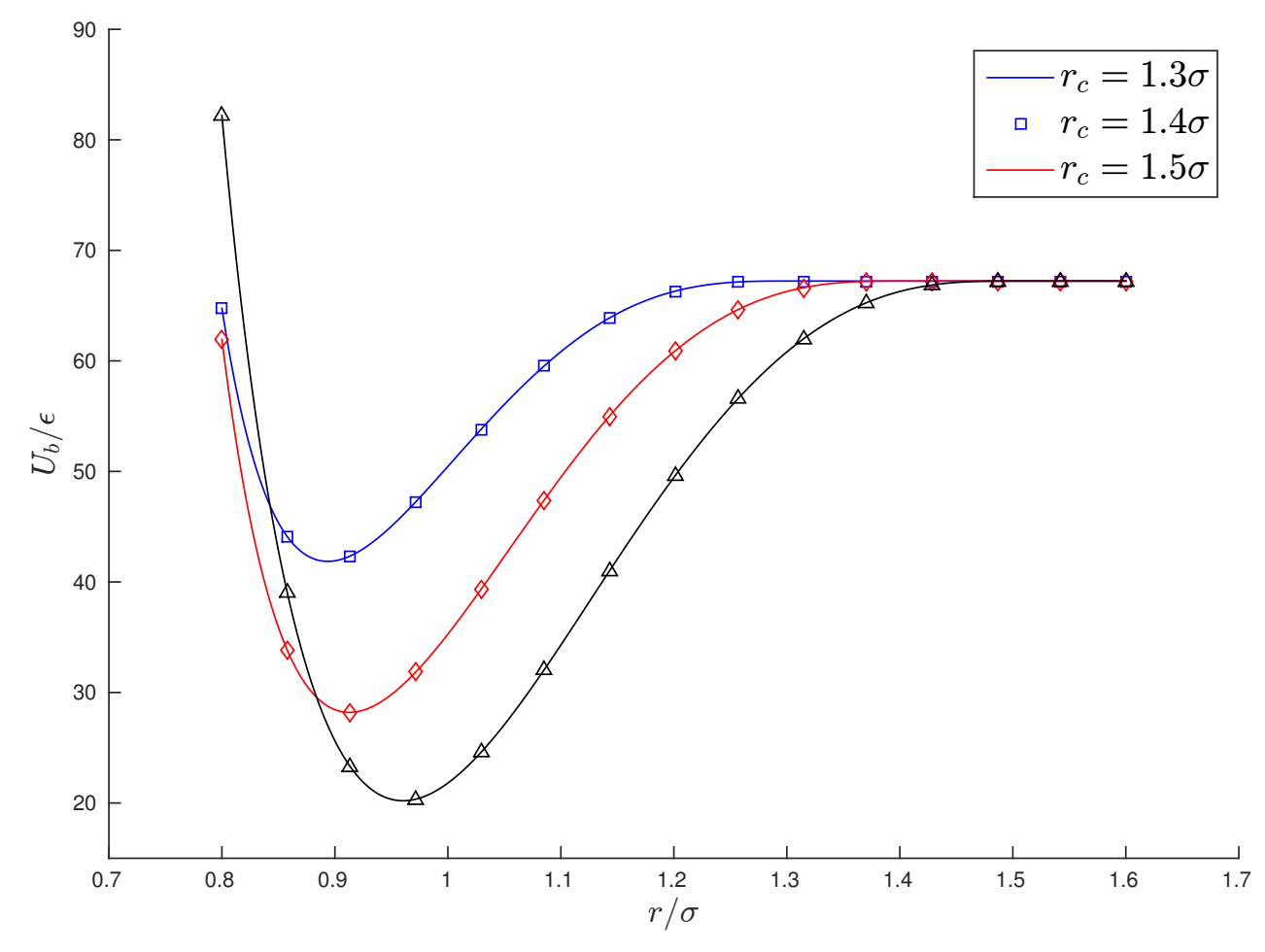

Figure 6.2: Bond potential curves.

The interface potential, $\phi_{i n t}$, is obtained by integration over the half space, as can be seen in Figure (6.3). The interaction energy of a molecule and a planar surface is the sum of its interactions with all the molecules in the body. A circular ring of cross-sectional area $d x d z$ and 
radius $x$ results in a volume of $2 \pi \beta x d x d z$, where $\beta$ is the density of the number of molecules in the solid.

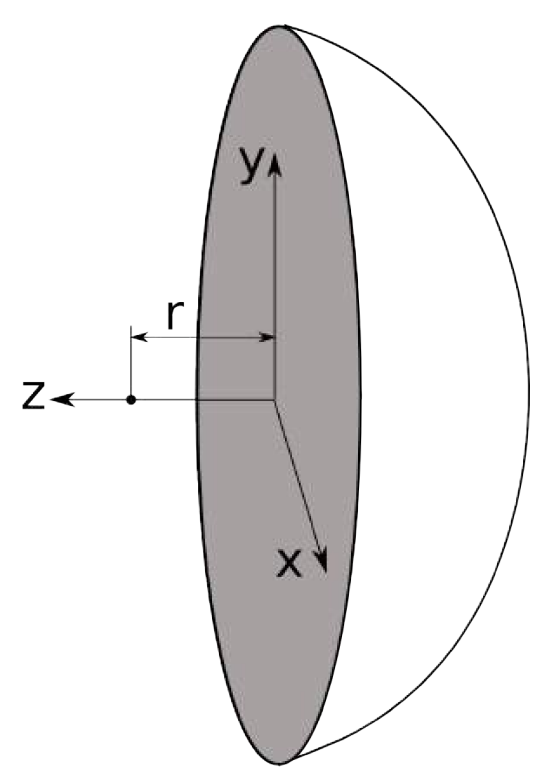

(a)

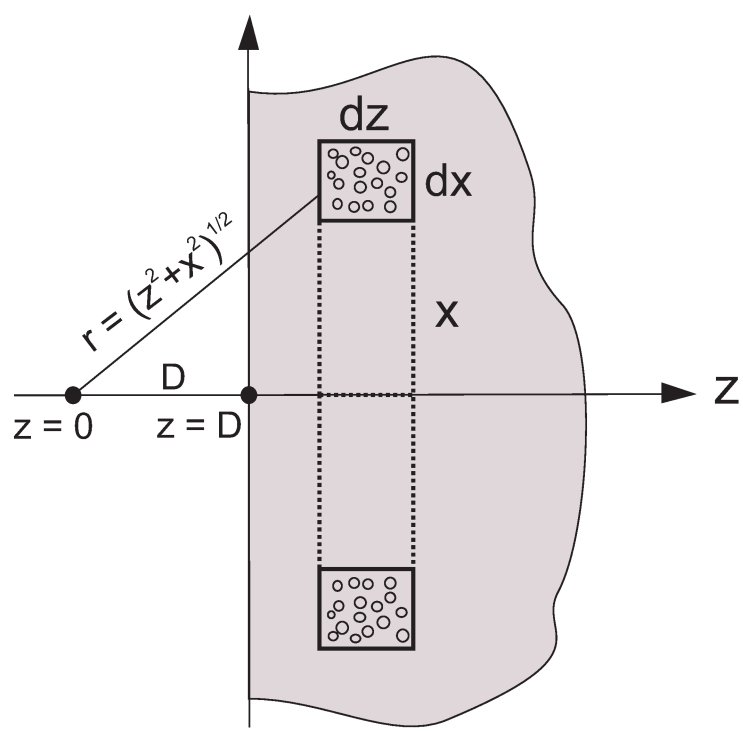

(b)

Figure 6.3: (a) Integration over half-space, (b) Molecule-body interaction in plane xz.

The net interaction energy for a molecule at a distance $D$ away from the surface will be, (ISRAELACHVILI, 1992),

$$
\phi_{\text {int }}=2 \pi \beta \int_{z=D}^{z=\infty} d z \int_{x=0}^{x=\infty} \phi_{g} x d x
$$

From Equation (6.3), the potential $\phi_{g}$ could be numerically integrated by Gaussian quadrature as follows,

$$
\phi_{\text {int }}=2 \pi \beta \int_{r}^{r_{c}} \int_{0}^{r_{c}} \phi_{g}(x, z) x d x d z=2 \pi \beta \sum_{p=1}^{n p} \sum_{q=1}^{n q} w_{p} w_{q} \phi_{g}\left(\xi_{p}, \xi_{q}\right) \xi_{q}
$$

where $n p, w_{p}$ and $\xi_{p}$ are the number of Gauss points, Gauss weights and Gauss coordinates for the $z$ variable, respectively. The same set of variables, but with the $q$ subscripts, represent the $x$ coordinate. 
The variations of the non-dimensional representation of $\phi_{\text {int }}$ with $r / \sigma$ is shown in Figure (6.4).

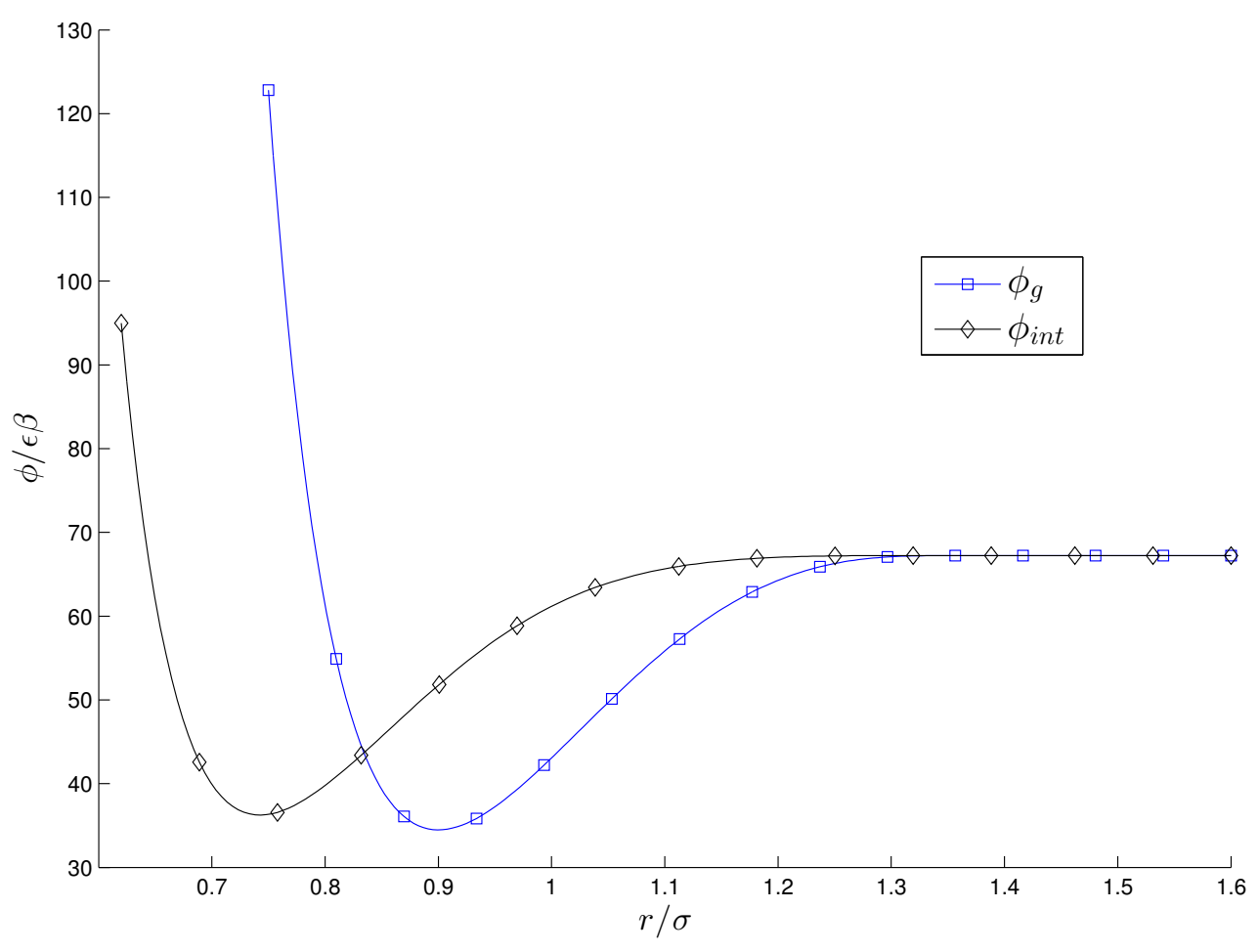

Figure 6.4: Bond potential curves.

The first derivative of the potential, which leads to interfacial forces, could be also obtained numerically. A simple estimation of the first derivative is obtained by applying finite difference, $f^{\prime}(x) \approx \frac{f(x+h)-f(x)}{h}$. Figure (6.5) show the non-dimensional representation of $\phi_{\text {int }}^{\prime}$.

The interfacial or depletion potential approach can be compared to a three-springs model, in which the springs are connected in series. The middle spring representing the cohesive interface. 


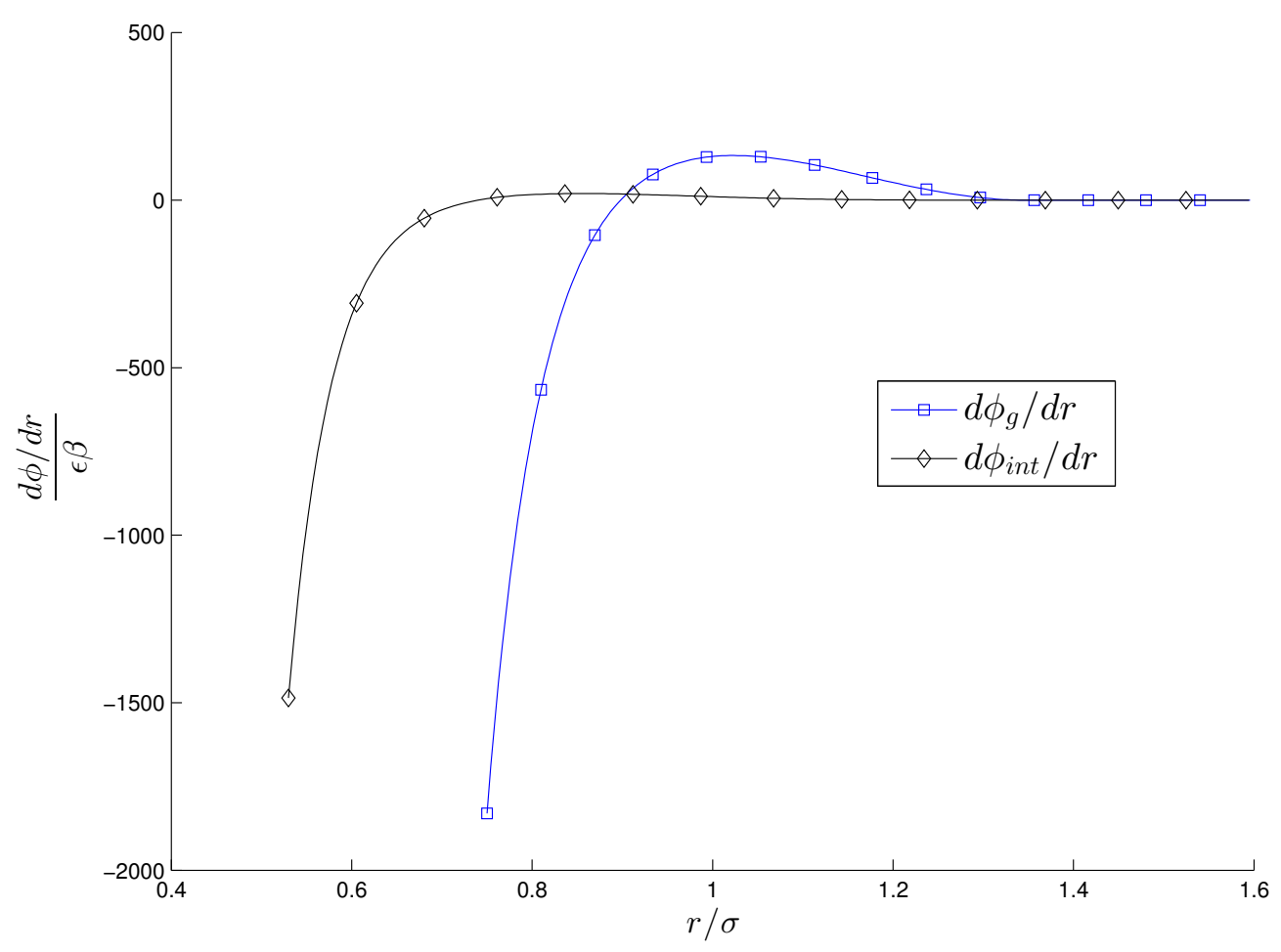

Figure 6.5: Derivative of the bond potential curves.

\subsection{Effective deformation gradient in the cohesive zone}

The cohesive zone is considered to be the weakest link in the homogeneous medium. There is present highly non-uniform local deformations due mainly to the presence of defects. In order to deal with the non-uniform deformation inside the finite-width cohesive zone, it is assumed that the deformation can be separated into two scales, (ZENG AND LI, 2012). The multi-scale displacement can be represented as

$$
\mathbf{u}=\overline{\mathbf{u}}+\mathbf{u}^{\prime}
$$

where $\bar{u}$ is the coarse, or Taylor, displacement field and $u^{\prime}$ is the fine scale fluctuation field. Equation (6.6) may be written for positions as follows,

$$
\mathbf{x}=\mathbf{X}+\mathbf{u}=(\mathbf{X}+\overline{\mathbf{u}})+\mathbf{u}^{\prime}=\overline{\mathbf{x}}+\mathbf{x}^{\prime}
$$


where $\overline{\mathrm{x}}$ and $\mathrm{x}$ are the position vectors of the deformed coarse and fluctuation scale, respectively; while, $\mathbf{X}$ is the position vector in the reference configuration. From Equation (6.7), it can be also deduced that,

$$
\begin{gathered}
\overline{\mathbf{x}}=\mathbf{X}+\overline{\mathbf{u}} \\
\mathbf{x}^{\prime}=\mathbf{u}^{\prime} .
\end{gathered}
$$

The deformation gradient in the coarse scale change more slowly than in the fine scale fluctuation field. Then, the general deformation field can be represented as

$$
\mathbf{x}=\mathbf{F}^{\mathbf{c}} \mathbf{X}+\mathbf{u}^{\prime}
$$

where $\mathbf{F}^{c}$ is the coarse scale deformation gradient, that can be obtained from the continuum displacement field along the cohesive zone. Moreover, $\mathbf{F}^{c}$ can be expressed using the HillMandel homogenization (HILL, 1972)

$$
\mathbf{F}^{c}=<\mathbf{F}>_{\Omega_{0}}=\frac{1}{\left|\Omega_{0}\right|} \int_{\Omega_{0}} \frac{\partial \mathbf{x}}{\partial \mathbf{X}} \mathrm{d} \Omega
$$

where $<\mathbf{F}>_{\Omega_{0}}$ represents the average deformation gradient in the cohesive zone over the volume $\Omega_{0}$. Substituting Equation (6.7) into Equation (6.10) and applying the divergence theorem leads to

$$
\overline{\mathbf{F}}^{c}=\frac{1}{\left|\Omega_{0}\right|} \int_{\Omega_{0}}\left(\frac{\partial \overline{\mathbf{x}}}{\partial \mathbf{X}}+\frac{\partial \mathbf{u}^{\prime}}{\partial \mathbf{X}}\right) d \Omega=\frac{1}{\left|\Omega_{0}\right|} \int_{\partial \Omega_{0}} \overline{\mathbf{x}} \otimes \mathbf{N} d s+\frac{1}{\left|\Omega_{0}\right|} \int_{\partial \Omega_{0}} \mathbf{u}^{\prime} \otimes \mathbf{N} d s
$$

where $\mathbf{N}$ is the normal vector of the cohesive zone boundary $\partial \Omega_{0}$. In order to account for rigid body motion, the value of $\mathbf{u}^{\prime}$ is set to zero for all material points in $\Omega_{0}$. Thus, the second term in Equation (6.11) vanishes. The deformation gradient $\overline{\mathbf{F}}^{c}$ can be calculated using the coarse scale response $\overline{\mathbf{x}}$ of the displacement field, as follows, 


$$
\overline{\mathbf{F}}^{c}=\frac{1}{\left|\Omega_{0}\right|} \int_{\partial \Omega_{0}} \overline{\mathbf{x}} \otimes \mathbf{N d} s
$$

The expression in Equation (6.12) can also be represented by an affine function of coordinates in the coarse scale domain $\Omega$, (ZENG AND LI, 2012)

$$
\overline{\mathbf{F}}=<\mathbf{F}>_{\Omega_{0}}=\overline{\mathbf{F}}^{c}:=\left.\frac{\partial \overline{\mathbf{x}}}{\partial \mathbf{X}}\right|_{\Omega}
$$

$\overline{\mathbf{F}}$ has nine unknown variables in a 3D problem. In order to solve for these unknowns, the deformation of at least three vectors must be known, (REN AND LI, 2013). Defining the coordinates of the undeformed field as $\mathbf{R}_{i}=\left(X_{i}, Y_{i}, Z_{i}\right)$; while the coordinates of the deformed field are $\mathbf{r}_{i}=\left(x_{i}, y_{i}, z_{i}\right)$. All nodal displacements can be calculated from the bulk elements, as shown in Figure (6.6). It is worth to point out that all nodal positions are regarding the physical nodes within our discontinuous quadratic element, as shown in Figure (3.2). Then, the relative position vectors can be easily obtained. Vectors $\mathbf{r}_{16}, \mathbf{r}_{27}$ and $\mathbf{r}_{38}$ are relative position vectors of the deformed position; while, $\mathbf{R}_{16}, \mathbf{R}_{27}$ and $\mathbf{R}_{38}$ are relative position vectors of the undeformed position.

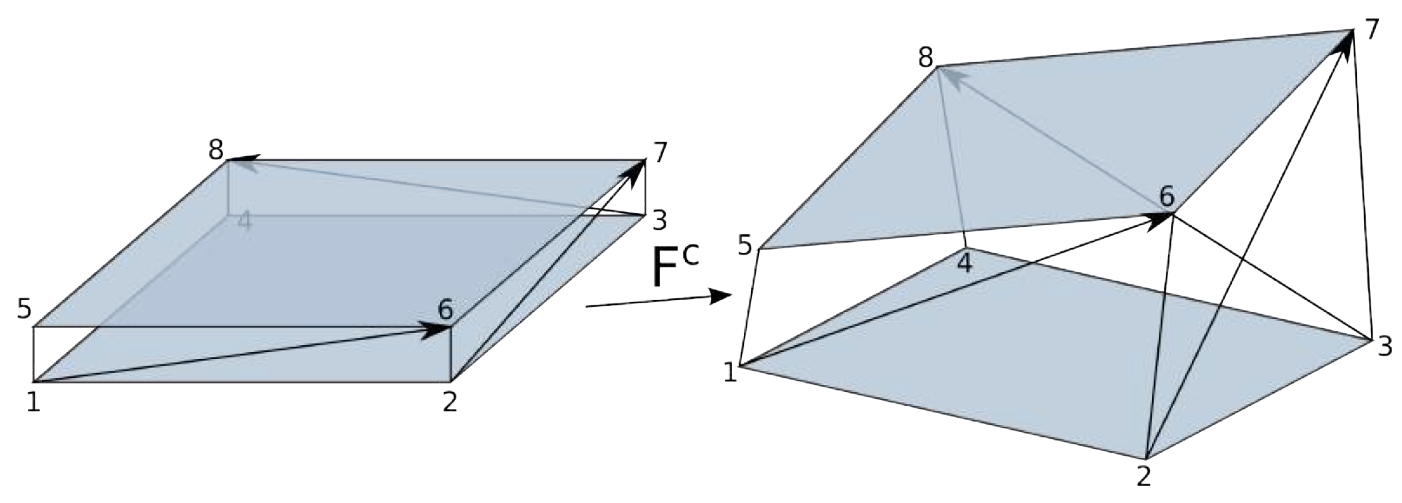

Figure 6.6: Nodal displacements for a interface 3D element.

The following geometric relationships can be obtained,

$$
\begin{aligned}
\mathbf{r}_{16} & =\overline{\mathbf{F}} \mathbf{R}_{16} \\
\mathbf{r}_{27} & =\overline{\mathbf{F}} \mathbf{R}_{27} \\
\mathbf{r}_{38} & =\overline{\mathbf{F}} \mathbf{R}_{38}
\end{aligned}
$$


The components of $\overline{\mathbf{F}}$ can be solved as

$$
\left(\begin{array}{l}
F_{11} \\
F_{12} \\
F_{13} \\
F_{21} \\
F_{22} \\
F_{23} \\
F_{31} \\
F_{32} \\
F_{33}
\end{array}\right)=\left[\begin{array}{ccc}
\mathbf{A}^{-1} & 0 & 0 \\
0 & \mathbf{A}^{-1} & 0 \\
0 & 0 & \mathbf{A}^{-1}
\end{array}\right]\left(\begin{array}{l}
x_{1}-x_{6} \\
x_{2}-x_{7} \\
x_{3}-x_{8} \\
y_{1}-y_{6} \\
y_{2}-y_{7} \\
y_{3}-y_{8} \\
z_{1}-z_{6} \\
z_{2}-z_{7} \\
z_{3}-z_{8}
\end{array}\right)
$$

where $\mathbf{A}$ is a constant second-order tensor in which their components are function of the nodal coordinates in the reference configuration, as follows,

$$
\mathbf{A}=\left[\begin{array}{lll}
\left(X_{1}-X_{6}\right) & \left(Y_{1}-Y_{6}\right) & \left(Z_{1}-Z_{6}\right) \\
\left(X_{2}-X_{7}\right) & \left(Y_{2}-Y_{7}\right) & \left(Z_{2}-Z_{7}\right) \\
\left(X_{3}-X_{8}\right) & \left(Y_{3}-Y_{8}\right) & \left(Z_{3}-Z_{8}\right)
\end{array}\right]
$$

The deformation gradient $\overline{\mathbf{F}}$, as shown above, depends only on the nodal displacements at the continuum coarse scale and can be obtained directly from the results of, e.g., a BEM analysis. Three random relative positions can be considered to solve the system of equations, Equation (6.15). However, in the present project, a more consistent criterion should be considered, such as, selecting the maximum deformation gradient obtained from two interface boundary elements.

\subsection{Cauchy-Born rule}

The Cauchy-Born rule, proposed by (ERICKSEN, 1984), is used to calculate the elastic energy within each element with information at the atomistic scale. It assumes that the deformation is uniform, then, the deformation gradient $\overline{\mathbf{F}}$ is constant in each element. An arbitrary deformed vector $\mathbf{r}_{i}$ in a unit cell or lattice structure can be found by mapping the corresponding reference un-deformed bond vector $\mathbf{R}_{i}$ 


$$
\mathbf{r}_{i}=\overline{\mathbf{F}} \mathbf{R}_{i}, \quad i=1,2, \ldots, n_{b}
$$

where $n_{b}$ is the total number of bonds in a unit cell. The bond vector $\mathbf{r}_{i}$ is the distance between the center atom in the unit cell to one of the atoms at the vertex. thus, the deformed bond length is a function of the deformation gradient in the unit cell and can be represented as $r_{i}=\left|\mathbf{r}_{i}\right|=$ $r_{i}(\overline{\mathbf{F}})$.

Due to the highly non-uniform deformation field, the Cauchy-Born rule cannot be directly applied. For a point in the cohesive zone, the first Piola-Kirchhoff tensor, in the deformed coordinates, can be expressed as, (ZENG AND LI, 2010),

$$
\mathbf{P}=\frac{\partial W}{\partial \overline{\mathbf{F}}}=\frac{\partial W}{\partial \overline{\mathbf{F}}^{c}}+\frac{\partial^{2} W}{\partial \overline{\mathbf{F}}^{c} \partial \overline{\mathbf{F}}^{c}}:\left(\frac{\partial \mathbf{u}^{\prime}}{\partial \mathbf{X}}\right)+O\left(\left|\frac{\partial^{2} \mathbf{u}^{\prime}}{\partial^{2} \mathbf{X}}\right|\right)
$$

Using the Hill-Mandel homogenization in Equation (6.18) leads to, (ZENG AND LI, 2010),

$$
\overline{\mathbf{P}}=\frac{1}{\left|\Omega_{0}\right|} \int_{\Omega_{0}} \mathbf{P} \mathrm{d} \Omega \approx \frac{\partial W}{\partial \overline{\mathbf{F}}^{c}}+\frac{\partial^{2} W}{\partial \overline{\mathbf{F}}^{c} \partial \overline{\mathbf{F}}^{c}}\left(\frac{1}{\left|\Omega_{0}\right|} \int_{\Omega_{0}} \frac{\partial \mathbf{u}^{\prime}}{\partial \mathbf{X}} \mathrm{d} \Omega\right)=\frac{\partial W}{\partial \overline{\mathbf{F}}^{c}}
$$

Applying the virtual work theorem, assuming that $\overline{\mathbf{F}}=\overline{\mathbf{F}}^{c}+\frac{\partial \mathbf{u}^{\prime}}{\partial X}$ and $\mathbf{u}^{\prime}=0$,

$$
\begin{gathered}
\mathbf{P}^{c}: \delta \overline{\mathbf{F}}^{c}=\overline{\mathbf{P}}: \delta \overline{\mathbf{F}}^{c} \\
\overline{\mathbf{P}}=\mathbf{P}^{c}=\frac{\partial W}{\partial \overline{\mathbf{F}}^{c}}
\end{gathered}
$$

where $W$ is the strain energy density. The homogenized Piola-Kirchhoff tensor $\overline{\mathbf{P}}$ can be determined from the homogenized deformation gradient $\overline{\mathbf{F}}^{c}$. Then, the Cauchy-Born rule can be used in the cohesive zones by computing only one unit cell. More details on the homegenization procedure can be found in (GALVIS AND SOLLERO, 2014), (WANG, 2013). The expression for the homogenized first Piola-Kirchhoff tensor in the cohesive zone is given as, (ZENG AND Li, 2010), (Li et al., 2012), (GAlvis AND SOllero, 2014), (GAlvis AND Sollero, 2016), 


$$
\overline{\mathbf{P}}=\frac{\partial W}{\partial \overline{\mathbf{F}}^{c}}=\frac{1}{\Omega_{0}^{c}} \sum_{i=1}^{n_{b}} \frac{\partial \phi_{\text {depl }}}{\partial \bar{r}_{i}} \frac{\mathbf{r}_{\mathbf{i}} \otimes \mathbf{R}_{i}}{\bar{r}_{i}}
$$

where $\Omega_{0}^{c}$ is the volume of the unit cell.

\subsection{Cohesive forces and failure criterion}

The cohesive force is highly dependent on the potential parameters, and as a result, dependent on the type of material. The cohesive traction forces along the boundary of the cohesive zone can be expressed as (ZENG AND LI, 2012),

$$
\mathbf{T}^{\text {cohe }}=\mathbf{P}^{c}\left(\overline{\mathbf{F}}^{c}\right) \cdot \mathbf{N}
$$

where $\mathbf{N}$ is the outward normal vector of two adjacent boundary elements. The failure due to decohesion can be assumed to occur when the cohesive force is less than a pre-set value $T_{\text {cohe }}$. This value can be obtained from imposed displacements - cohesive force curves, see Figure (6.9), Figure (6.10) and Figure (6.11), and when the intermolecular distance is higher than a previously set value $r_{\text {crit }}$ (ZENG AND Li, 2012), (GALVIS AND SOLLERO, 2014),(GALVIS AND SOLLERO, 2016). The latter being the main failure criterion of the present study. A flowchart of the entire failure progression process is shown in Figure 6.7. For convenience, a failure index (FI) is introduced as,

$$
F I=\frac{r}{r_{\text {crit }}}
$$

where values of $F I=1$ means that failure is present. 


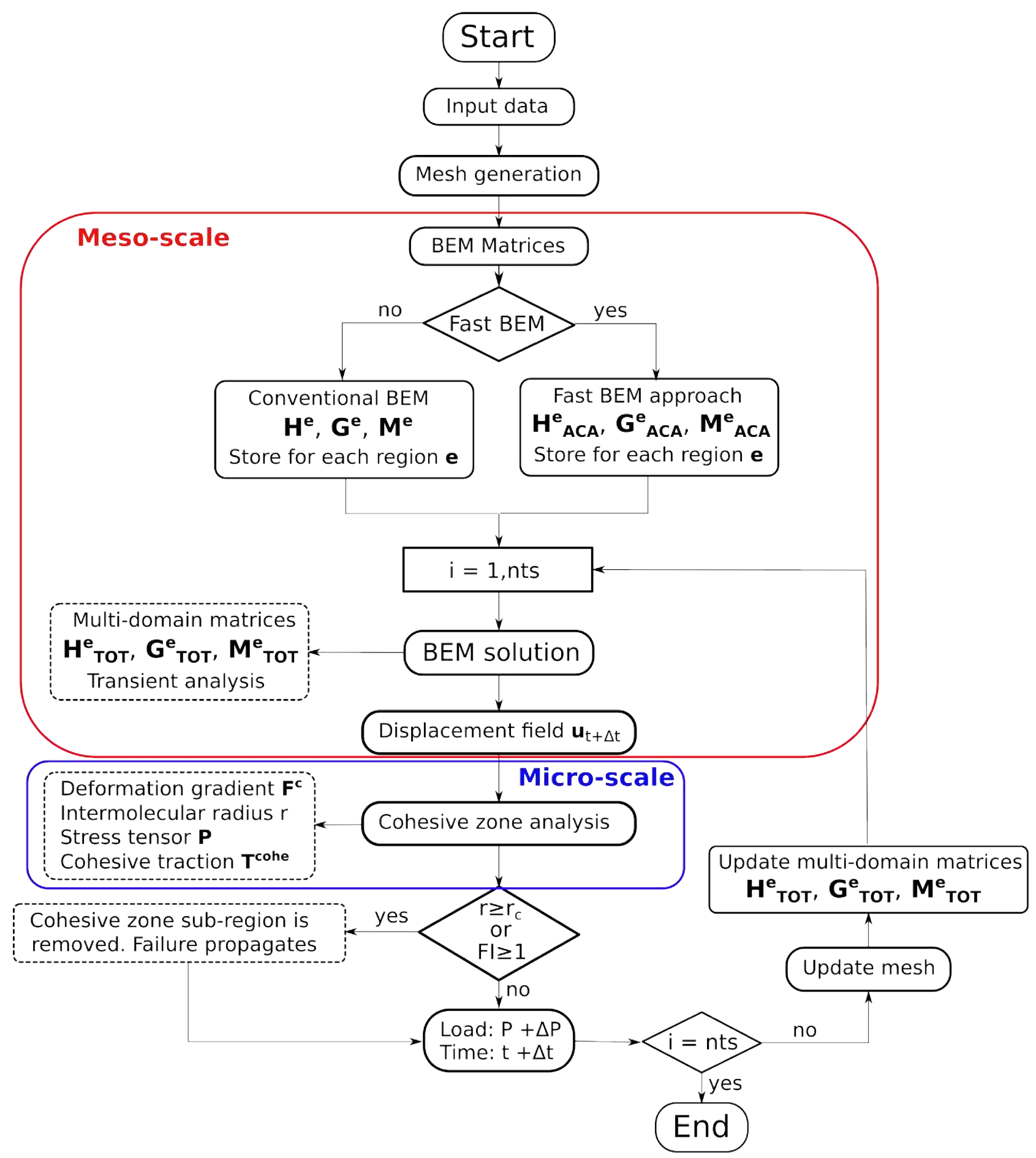

Figure 6.7: Multi-scale analysis flowchart.

The FI is adopted for its simple implementation into the computational code, besides giving a better failure visualization of the results in the post-processing section. 
Moreover, the cohesive traction forces can be obtained from Equation (6.22) for different imposed displacements. For illustration, a cohesive volume of $0.1 \times 0.1 \times 0.05$ and displacements of 0.001 were used. Material properties are the same as presented by (YANG et al., 2014) for an EPN-BPA epoxy. The decomposition of traction forces for three imposed displacements is shown in Figure (6.8).

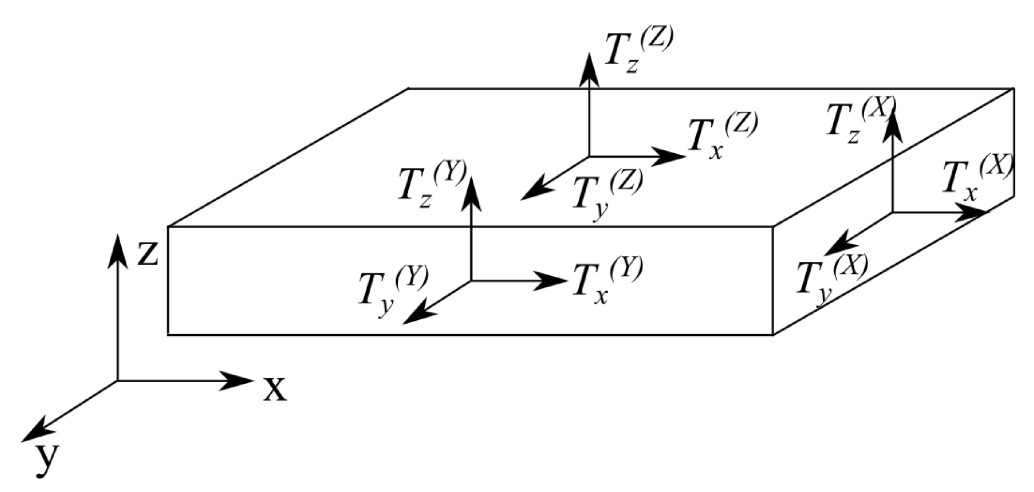

Figure 6.8: Cohesive traction forces components.

where $T_{i}^{j}$ represent the traction force in the $i$ coordinate axis due to an imposed displacement in the $j$ coordinate axis. The displacements in a $j$ coordinate axis are imposed in the corresponding opposite normal planes in the multi-scale cohesive sub-region. The cohesive forces as well as the imposed displacements are shown in: Figure (6.9) for a displacement in the $X$ coordinate axis; Figure (6.10) for a displacement in the $Y$ coordinate axis and Figure (6.11) for a displacement in the $Z$ coordinate axis 


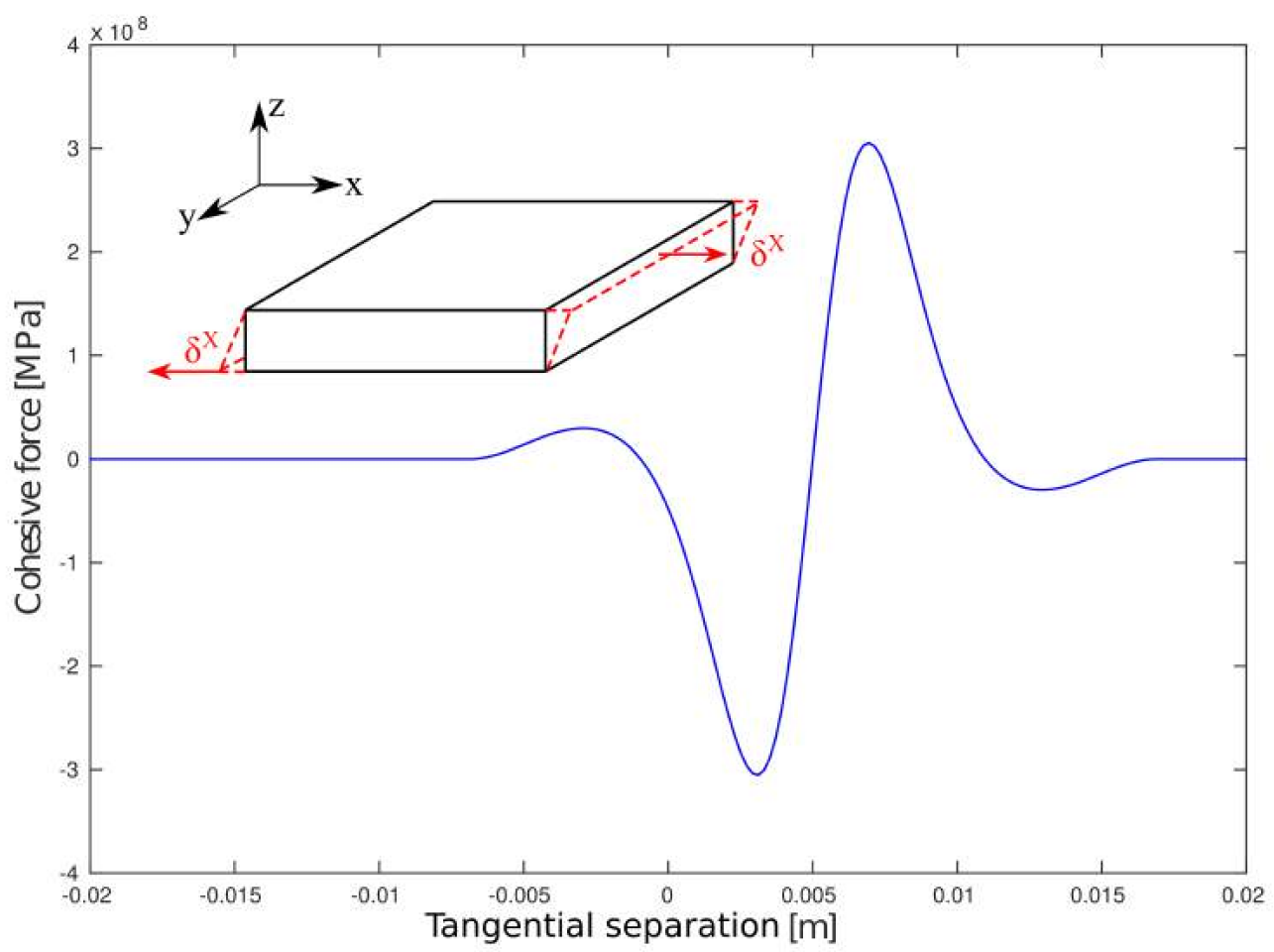

Figure 6.9: Cohesive normal force, when the imposed displacement is in the $X$ coordinate axis. 


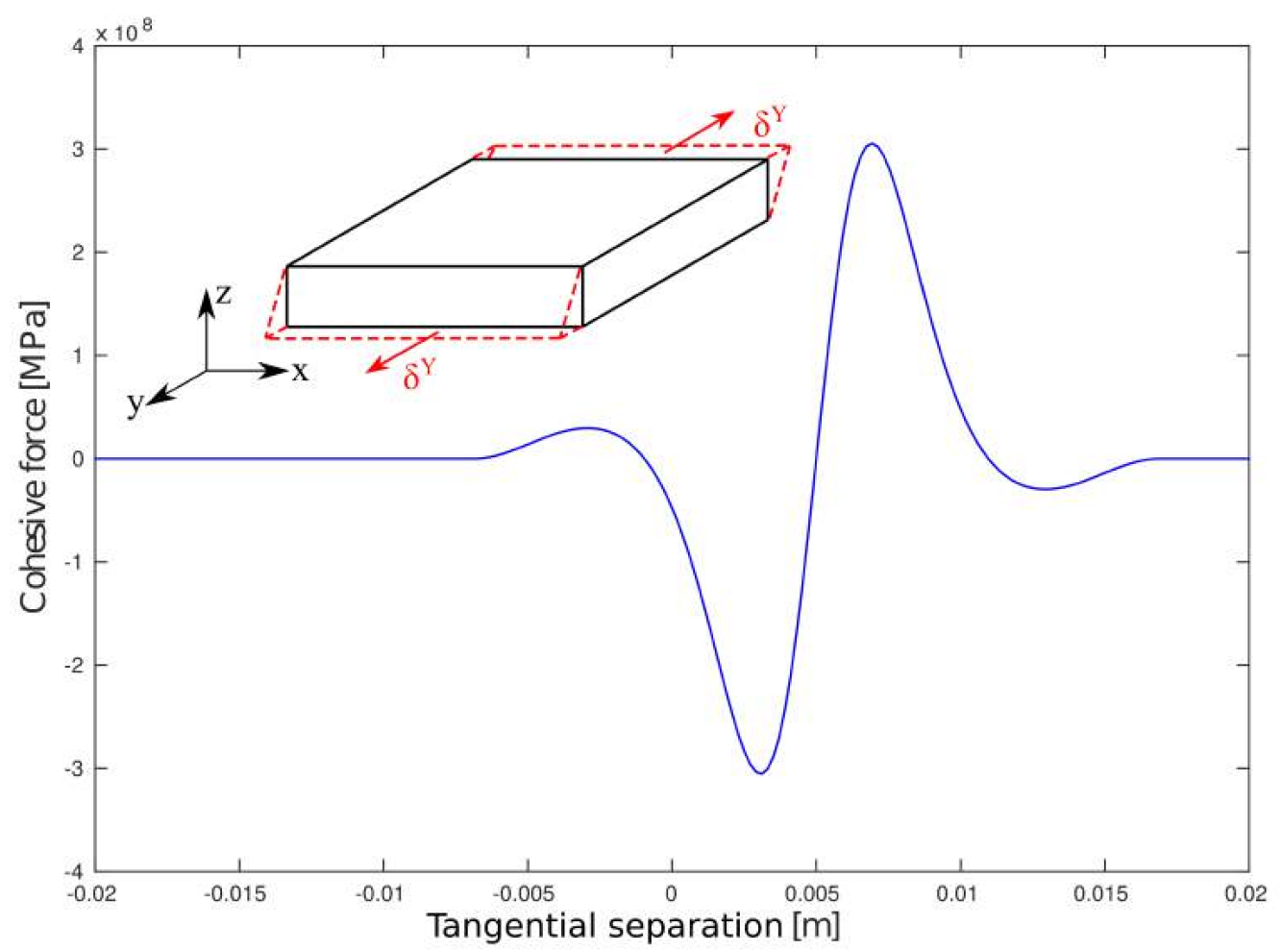

Figure 6.10: Cohesive normal force, when the imposed displacement is in the $Y$ coordinate axis. 


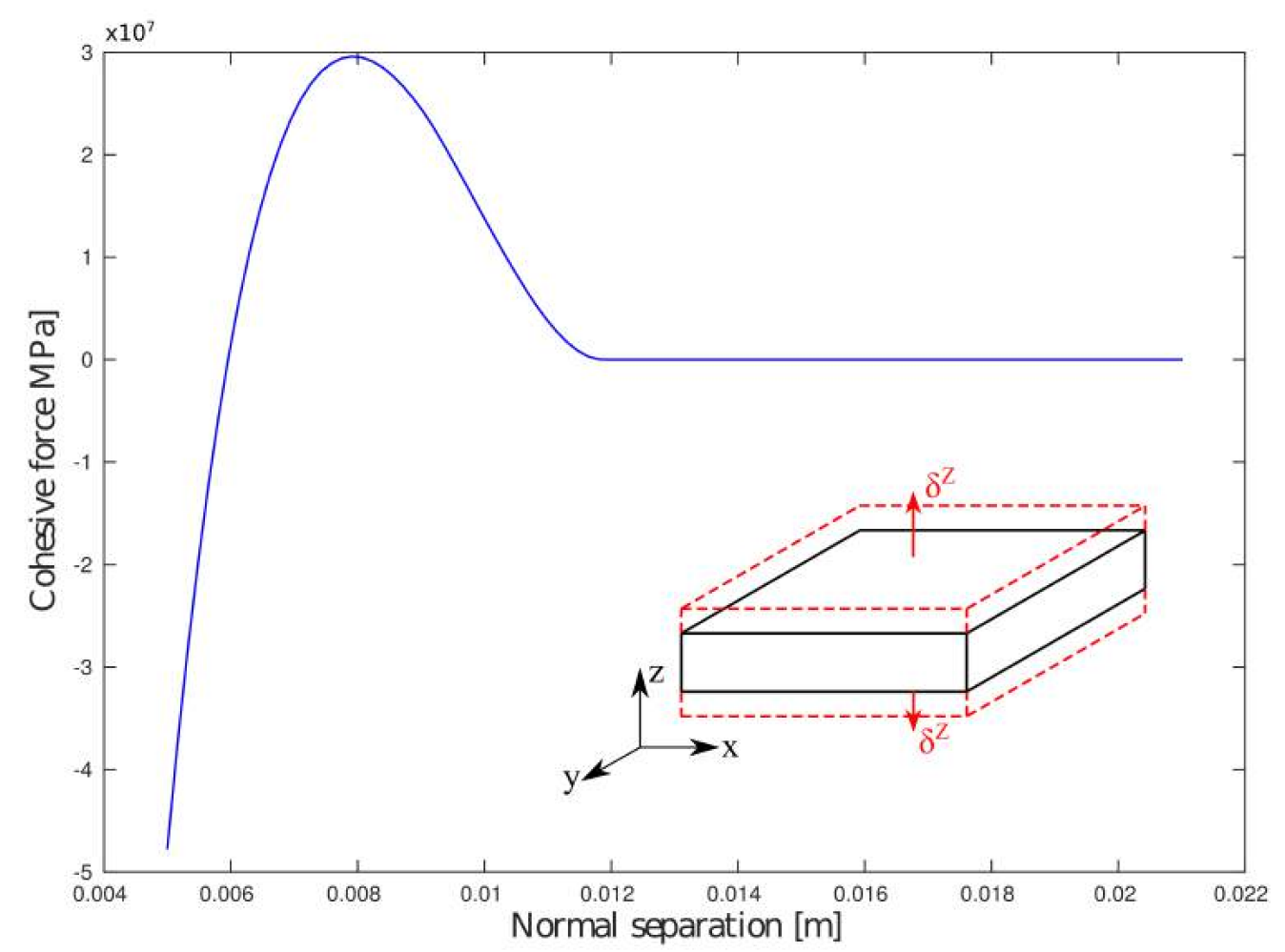

Figure 6.11: Cohesive normal force, when the imposed displacement is in the $Z$ coordinate axis.

\subsection{Numerical examples}

In this section, the laminate composite model, implementation issues and three numerical examples are presented. First, a numerical model for analysing laminate composites is shown. In the model, fibers and the matrix can have different materials; in addition, the generally anisotropic fundamental solutions are used allowing to test real material properties in a laminate composite. Some implementation issues are discussed. Three numerical examples are then analysed in order to show the applicability of the multi-scale failure criterion described in the previous section. The first example, Example A, shows the propagation of a corner initial failure within a $0^{\circ} / 0^{\circ}$ laminate composite, when the load is applied in the fiber plane. The second example, Example B, shows the propagation with a similar initial failure, but applied to a fiber composite. The load is in the transversal direction ( $z$ coordinate axis) in this case. Finally, Example $\mathrm{C}$, shows the propagation of failure in a laminate composite with an initial failure at the center of the interface between the laminae. The load in this last example is along the fiber direction ( $x$ coordinate axis). In all the cases analysed, for the Fourier series representation of the fundamental solutions in the BEM analysis, $\alpha=16$ and $k=64$ were used throughout. It is 
worth to point out that due to computational size restrictions, in the numerical examples, fiber tows are modelled instead of fibers. A fiber tow is an untwisted bundle of continuous filaments, which can reach the numbers of several thousands in a real laminated composite.

\subsubsection{Laminate composite model}

First, the laminate composite model is presented. A general mesh generator for its different components was implemented from scratch. The programming language used was Fortran 90; for compiling the code, the GNU compiler gfortran was used. Moreover, some routines were parallelized using the OpenMP interface. As the multi-domain generates a sparse system matrix, the PARDISO solver was used for solving the system of algebraic expressions.

In this model, the fibers can have the $0^{\circ}$ or $90^{\circ}$ directions on the laminae. The dimensions of the fibers and mesh are totally defined by the user. As a consequence, the fraction volume can also be imposed. The multi-scale criterion is only applied in the interface between laminae. Each multi-scale region is defined by a six-element cube volume in which the multi-scale can be properly applied. Moreover, a re-finement of these regions can be also established. Some of the input variables of the program are,

○ $\mathrm{Mat}_{m}, \mathrm{Mat}_{f}$ : Materials for the matrix and fibers, respectively.

○ $L_{x}, L_{y}, l_{i}$ : Laminae length on the $x$ and $y$ directions; and interface thickness, respectively.

○ $L_{z 1}, L_{z 2}$ : Thickness for the bottom and top laminae, respectively.

○ $r_{a}, L_{a}$ : Fiber radius and length, respectively. The sub-index $a$ indicates if the fibers correspond to the bottom lamina, $a=1$; or the top lamina, $a=2$.

$\circ n_{x}, n_{y}$ : Number of elements for the mesh discretization in the $x, y$ directions, respectively.

○ $n_{z 1}, n_{z 2}$ : Number of elements for the mesh discretization in the $z$ direction for the bottom and top laminae, respectively. As the bottom and top laminae can have different thickness, the discretization in the $z$-direction can also be different.

- $n u m f_{a}$, numz $f_{a}$ : Number of total fibers on the $x y$ plane and number of fiber layers on the $z$ direction, respectively. (Bottom lamina $a=1$, top lamina $a=2$ ).

- ntheta $f_{a}, n z f_{a}$ : Number of elements on the fiber in the radial direction and in the fiber length direction, respectively. (Bottom laminae $a=1$, top laminae $a=2$ ). 
○ $X_{0}, X_{1}$ : Coordinates that define the laminate lengths in the $x$ and $y$ directions.

○ $X r_{0}, X r_{1}$ : Coordinates that define the region to be refined, Figure 6.12 (b).

○ $n i v_{x}$, niv $_{y}$ : Discretization level of the refined section.

Figure 6.12 shows the model applied to a laminate composite with a $0^{\circ} / 90^{\circ}$ distribution and 4 fibers per laminae. The numeric parameters used for this example are,

○ Laminate geometry: $L_{x}=100, L_{y}=100, L_{z 1}=3, L_{z 2}=3, L_{i}=0.3$.

○ Fiber geometry: $r_{1}=r_{2}=0.5, L_{1}=L_{2}=80$.

○ Laminate mesh: $n_{x}=n_{y}=2, n_{z 1}=n_{z 2}=1$.

○ Fiber mesh: num $_{1}=$ num $_{2}=4$, num $_{1}=$ num $_{2}=1$, ntheta $f_{1}=$ ntheta $f_{2}=4$, $n z f_{1}=n z f_{2}=4$.

○ Interface refinement: $X r_{0}=(50,50,0), X r_{1}=(100,100,0)$

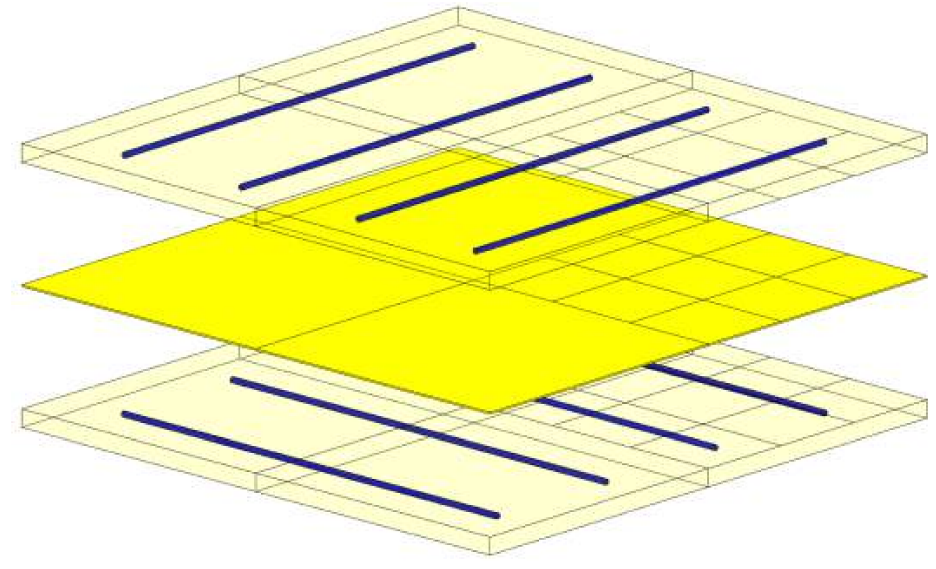

(a)

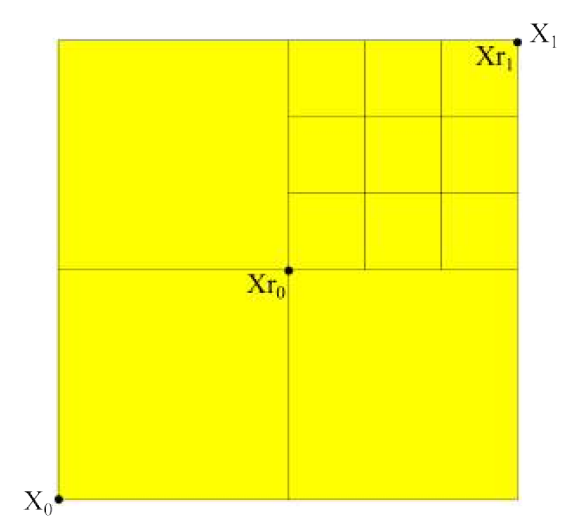

(b)

Figure 6.12: (a) Laminate composite model with a $0^{\circ} / 90^{\circ}$ distribution and 4 fibers per lamina; (b) Discretization of the interface at the laminate composite.

The laminate composite model can be further adapted to account for other types of composite, e.g., short-fiber, long-fiber, non-symmetric laminates, among others. Some examples of the model use as well as the failure criterion, is shown below. 


\subsubsection{Implementation issues}

Special care has to be taken in order to guarantee a correct failure progression on the interface of the composite. The most important modeling issue found was the discretization transition among the elements. A laminate composite encastred on its left side and subjected to a relatively high dynamic load to its opposite side is shown for illustration. The boundary conditions can be observed in Figure 6.13.

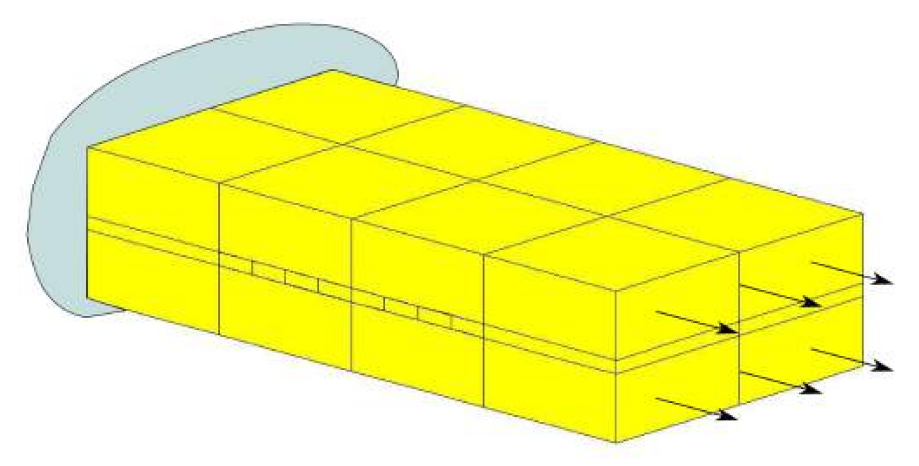

Figure 6.13: Boundary conditions of the composite laminate.

The failure is expected to initiate at the middle of the laminate composite interface. However, high deformation gradients lead to failure at the transition between the discretized area and the non-discretized area within the interface, are as shown in Figure 6.14. 


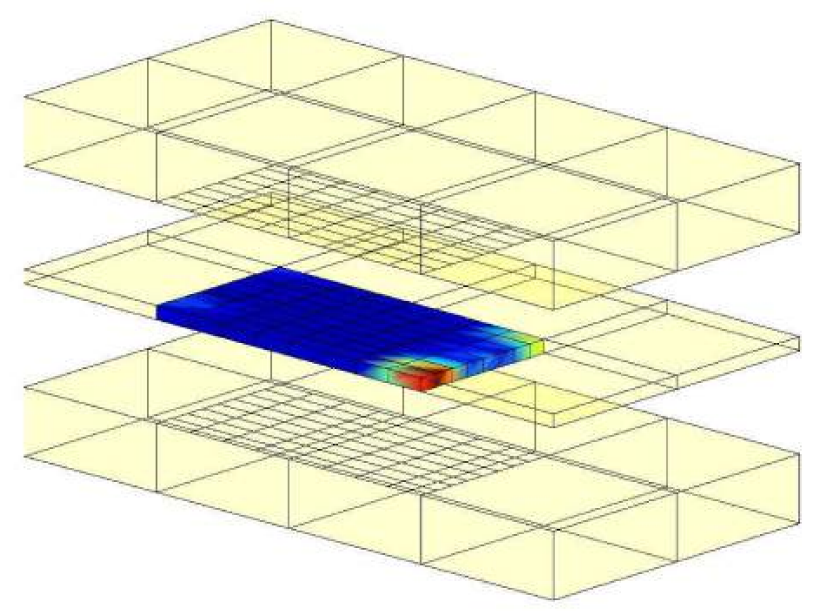

(a)

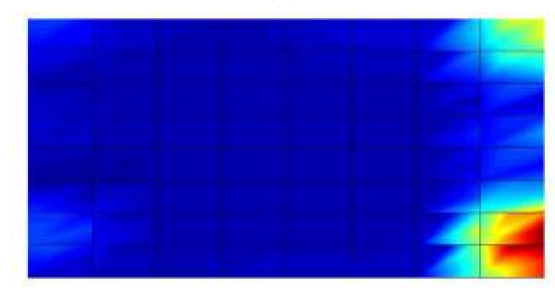

(b)

Figure 6.14: (a) Laminate implementation issue due to discretization, (b) Laminate interface.

A good discretization with a gradual transition of the size of the elements is required in order to correctly analyse the failure. Moreover, it should be noted that proper boundary conditions must be applied in order to guarantee failure progression. For example, failure progression may not be reached if the laminate is loaded in the fiber direction and the load is not high enough. In practice, shear and high loads should be considered in order to reach failure in a faster and more realistic manner.

\subsubsection{Example A: Laminate composite}

In this example the multi-scale failure criterion is tested for a laminate composite with a $0^{\circ} / 0^{\circ}$ fiber distribution. The laminate is loaded in the $x$ and $y$ coordinate directions, the boundary conditions are shown in Figure 6.15(a). An initial failure, by considering material absence, is set at the interface corner of the composite, as can be seen in Figure 6.15(b). An initial load, $P_{0}$, of $1400 \mathrm{MPa}$ and final load, $P_{\max }$, of $6200 \mathrm{MPa}$ are considered. The load process is completed in 187 time steps of $\Delta t=5 \times 10^{-4} \mathrm{~s}$. 


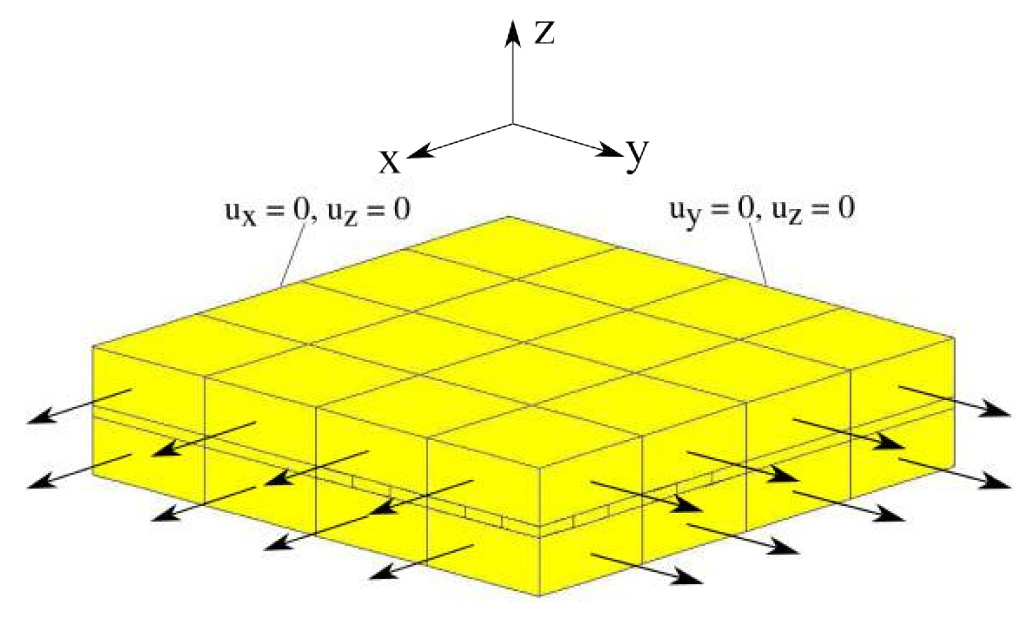

(a)

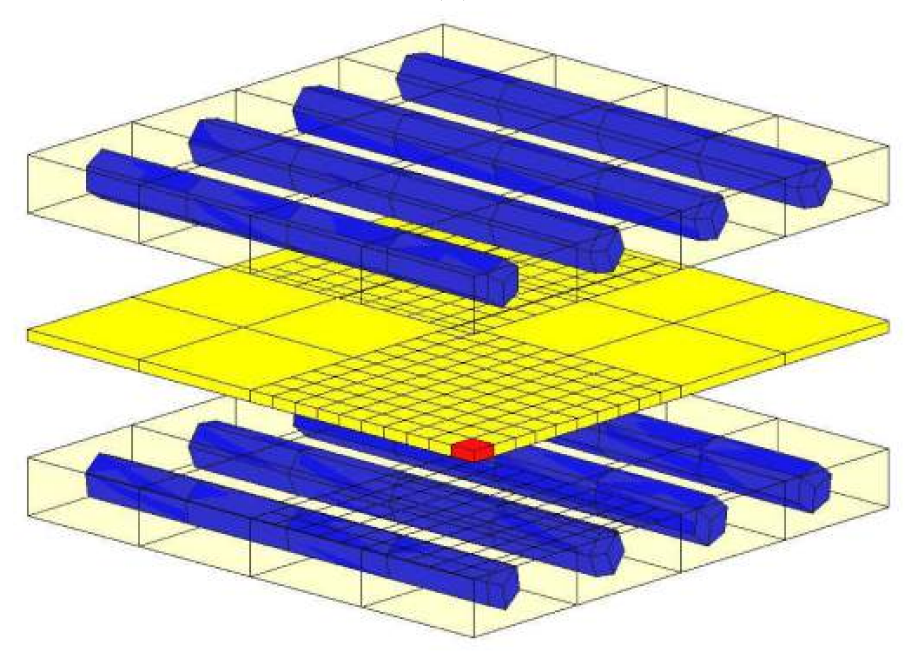

(b)

Figure 6.15: Laminate composite with a $0^{\circ} / 0^{\circ}$ fiber distribution; (a) Boundary conditions, (b) Fiber discretization and initial failure (red).

The matrix material is taken to be an EPN-BPA epoxy. The epoxy is considered as isotropic with an elasticity modulus of $E=1.868 \mathrm{GPa}$ and a Poisson's ratio of $\nu=0.416$. The parameters for the intermolecular potential for this material are defined in (YANG et al., 2014). $\sigma=4.383 \AA, \epsilon=1.519 \mathrm{kcal} / \mathrm{mol}=1.047 \times 10^{-20} \mathrm{~J}\left(1 \AA=10^{-10} \mathrm{~m}, 1 \mathrm{kcal} / \mathrm{mol}=0.043 \mathrm{eV}\right.$, $1 \mathrm{eV}=1.6022 \times 10^{-19} \mathrm{~J}$ ). For the fibers, a Carbon fiber AS4 is considered. The fiber material is considered as transversely isotropic, (SODEN et al., 1998), with the following properties, $E_{1}=225 \mathrm{GPa}, E_{2}=15 \mathrm{GPa}, G_{12}=15 \mathrm{GPa}, \nu_{12}=0.2$ and $G_{23}=7 \mathrm{GPa}$.

The laminate composite was modelled with 1120 quadratic discontinuous elements and 8960 quadratic discontinuous nodes, resulting in 26880 DOFs. A total of 75 regions were cre- 
ated, from which, 64 regions were used for the multi-scale approach application.

The ACA was applied for the generation of the BEM matrices. The ACA error tolerance is set to $\epsilon_{c}=10^{-4}$. The maximum number of elements per cluster was set to 20 and the admissibility parameter, $\eta$, to 0.9 . A total of 68 clusters and 507 blocks were created, from which 200 were admissible pairs. The ACA was applied to three regions: The bottom matrix, the top matrix and the non-discretized area of the laminate composite interface. The ACA does not work as well with a small number of elements, consequently, it cannot be applied to the remaining regions, such as the fibers and the multi-scale interface regions. For this example, the conventional BEM generated all matrices in 4988s, while the ACA generated them in $3662 \mathrm{~s}$, representing $26.6 \%$ reduction in processing time. The total analysis time was approximately 5 hours. This example was performed on the Bigmem node from the Kahuna cluster that belongs to the CEPID project funded by FAPESP. The Bigmem node has 64 cores and 1TB RAM memory. It requested 32 cores for the present example. The OpenMP interface was used for multi-processing among threads.

Figures 6.16, 6.17 and 6.18 show the multi-scale criterion applied to a pre-set discretized area within the laminated composite for different time steps. The value for the criterion goes from 0 (blue) to 1 (red), the latter representing failure. Figure 6.16 (a-c) shows the multi-scale criterion for time steps 1, 50 and 70, respectively; Figure 6.17 (a-c) shows the multi-scale criterion for time steps 100, 111 and 128, respectively and Figure 6.18 (a-c) shows the multi-scale criterion for time steps 148,164 and 180, respectively. It is worth to point out that the progression of the failure tends to follow the direction where the laminate is less resistant, in this case, the transverse direction.

In this example the failure tended to propagate in the $x$ coordinate direction, transverse to the fiber axis direction, $y$ coordinate axis. This failure propagation path is expected due to fiber and load directions. In the case of an homogenized isotropic material, failure would be expected to propagate in the radial direction. The Fast BEM approach result in a reduction of $26.6 \%$ in processing time, being an acceptable reduction time for the size of the problem. Better reductions are expected for bigger problems. As mentioned on the Section 6.6.2, one of the main implementation issues is the correct discretization and transition among neighbour elements. A brief previous mesh study was necessary to ensure the convergence of the results. 

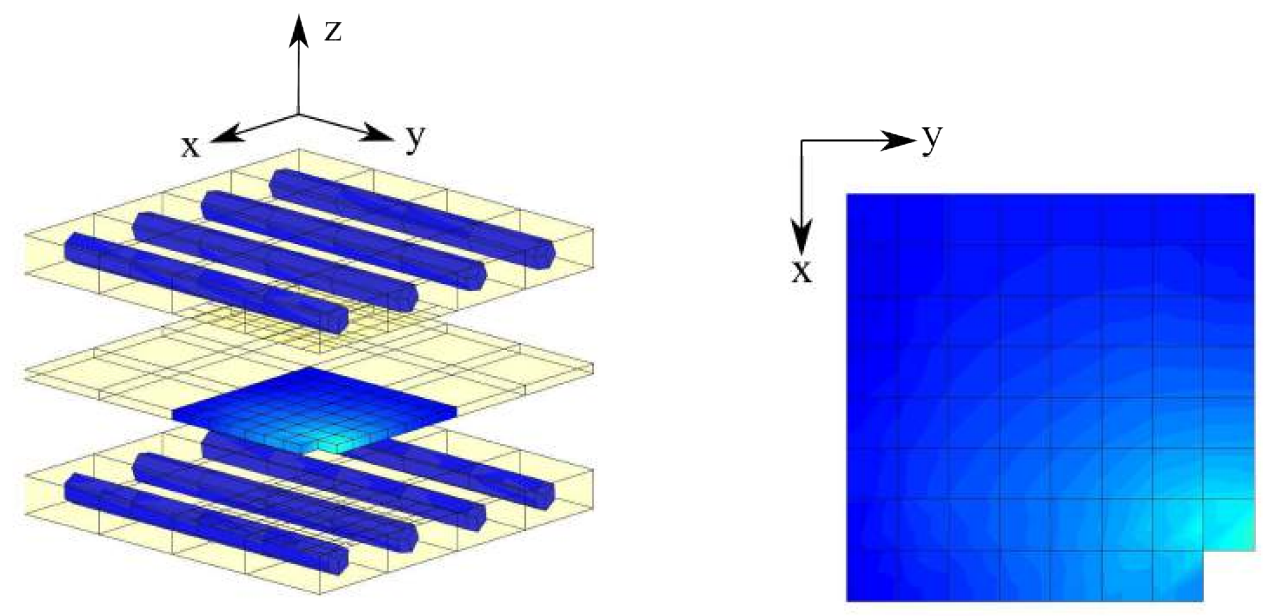

(a)
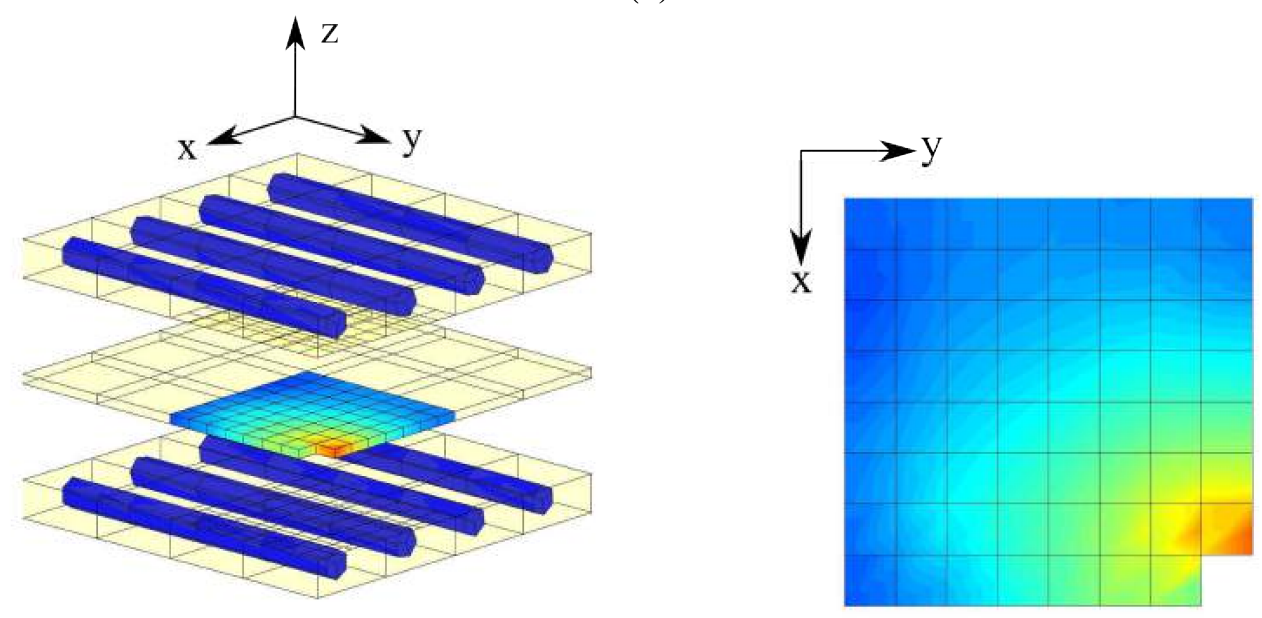

(b)
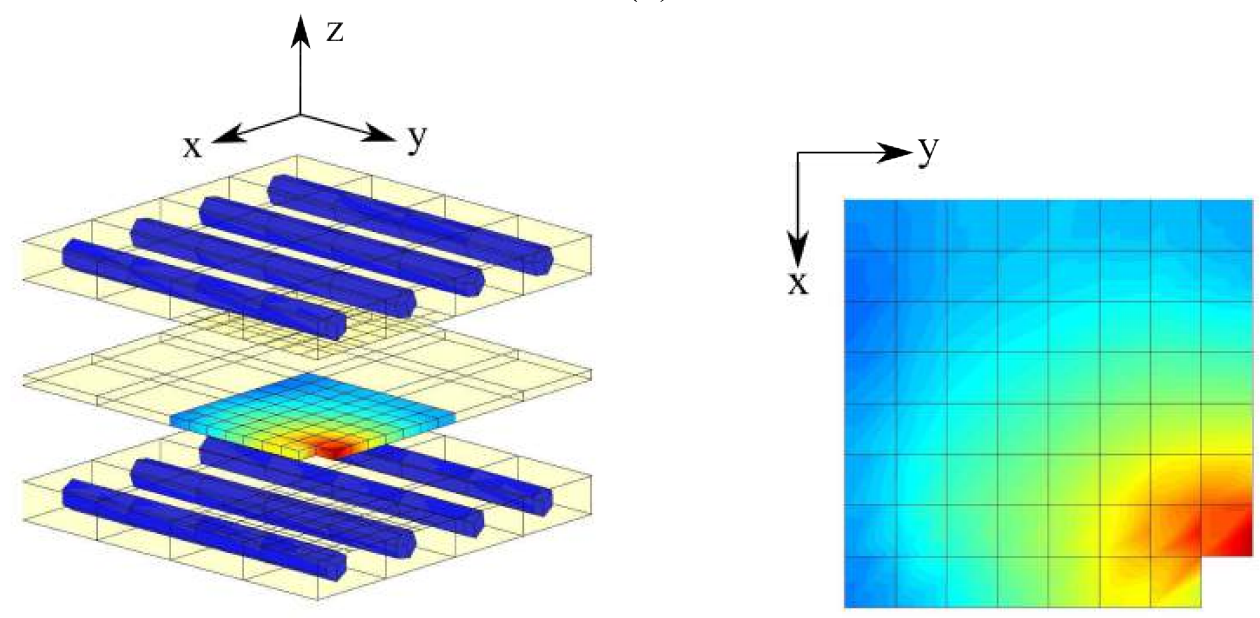

(c)

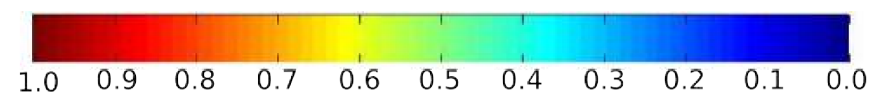

Figure 6.16: Multi-scale failure: (a) Time step 1, $P=1400 \mathrm{MPa}, t=0 \mathrm{~ms}$; (b) Time step 50, $P=2665 \mathrm{MPa}, t=24 \mathrm{~ms}$; (c) Time step 70, $P=3181 \mathrm{MPa}, t=34.5 \mathrm{~ms}$ 

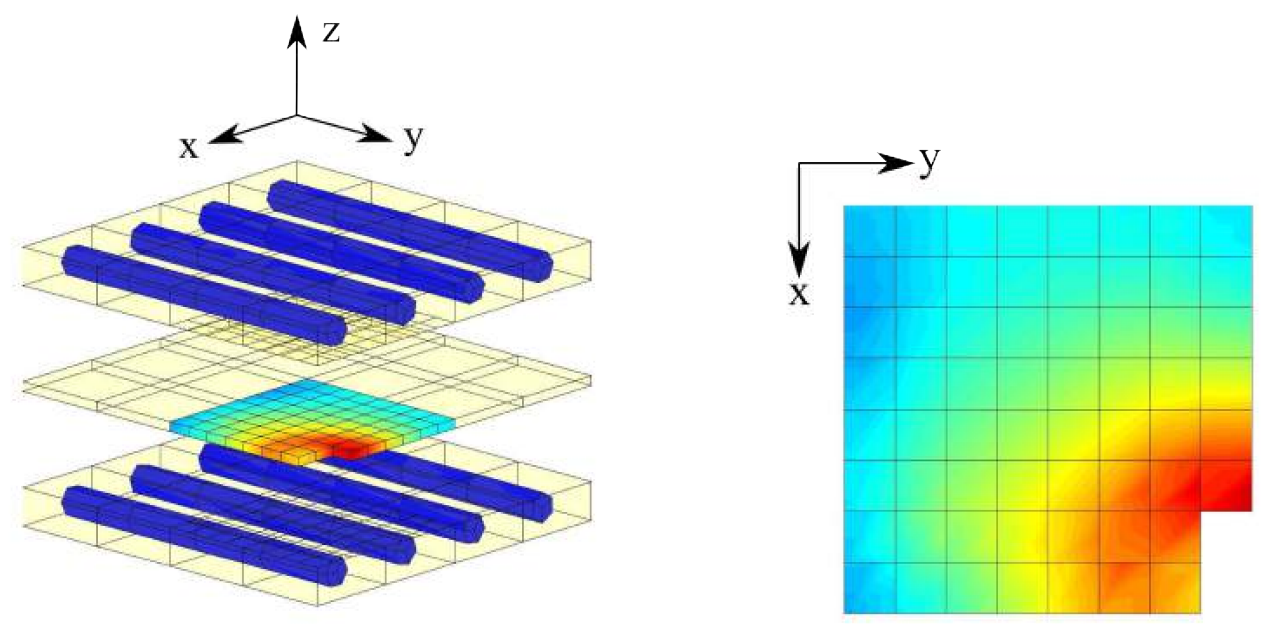

(a)
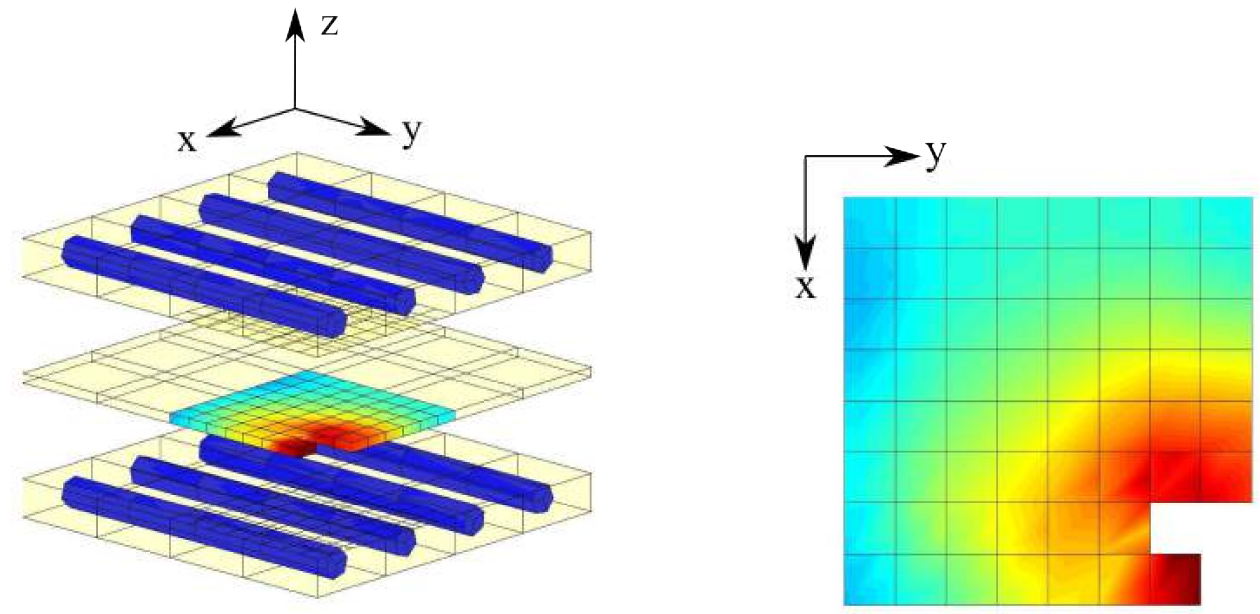

(b)
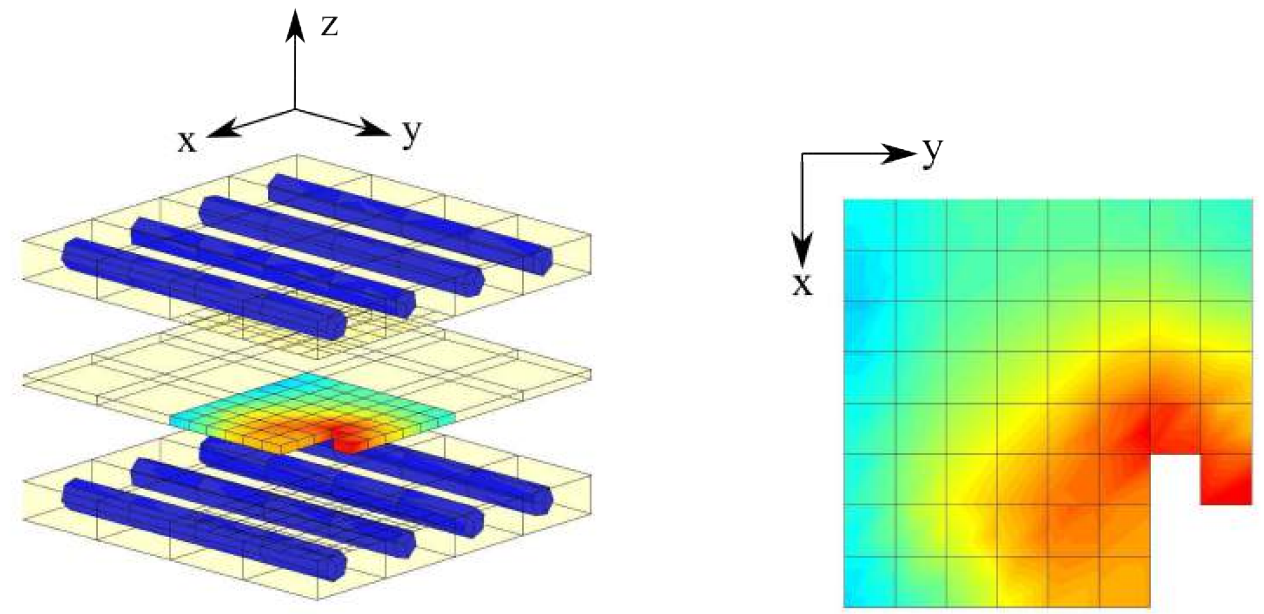

(c)

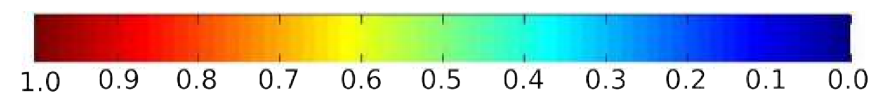

Figure 6.17: Multi-scale failure: (a) Time step 100, $P=3955 \mathrm{MPa}, t=49.5 \mathrm{~ms}$; (b) Time step $111, P=4239 \mathrm{MPa}, t=55 \mathrm{~ms}$; (c) Time step $128, P=4677 \mathrm{MPa}, t=63.5 \mathrm{~ms}$ 

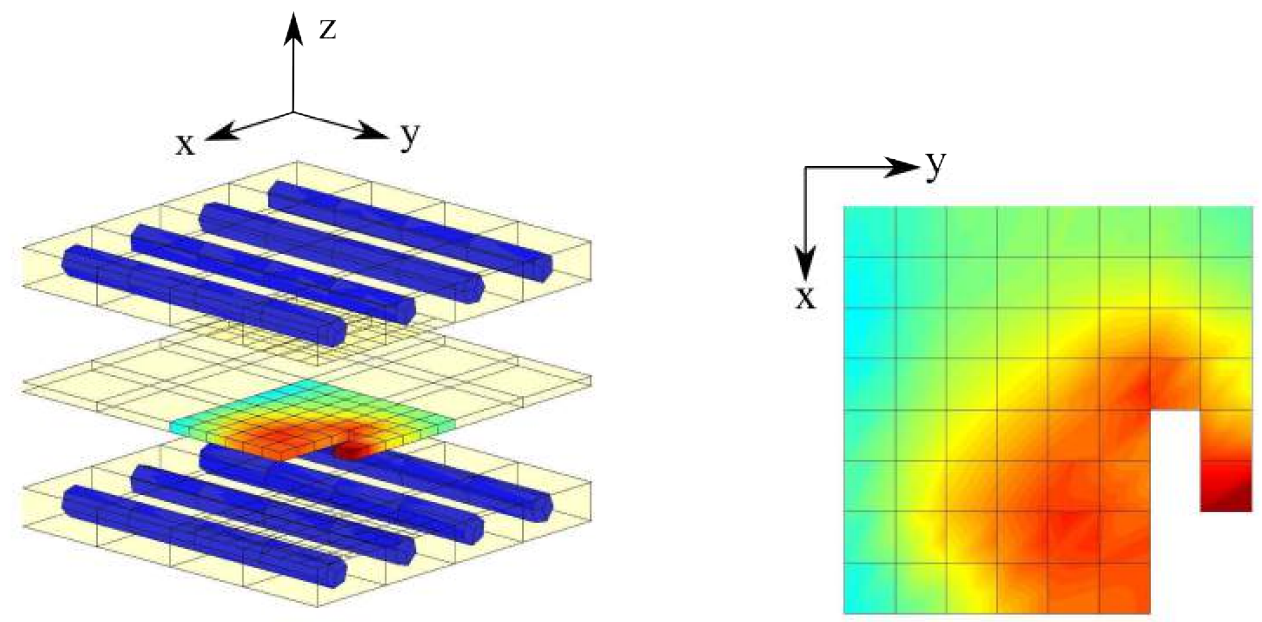

(a)
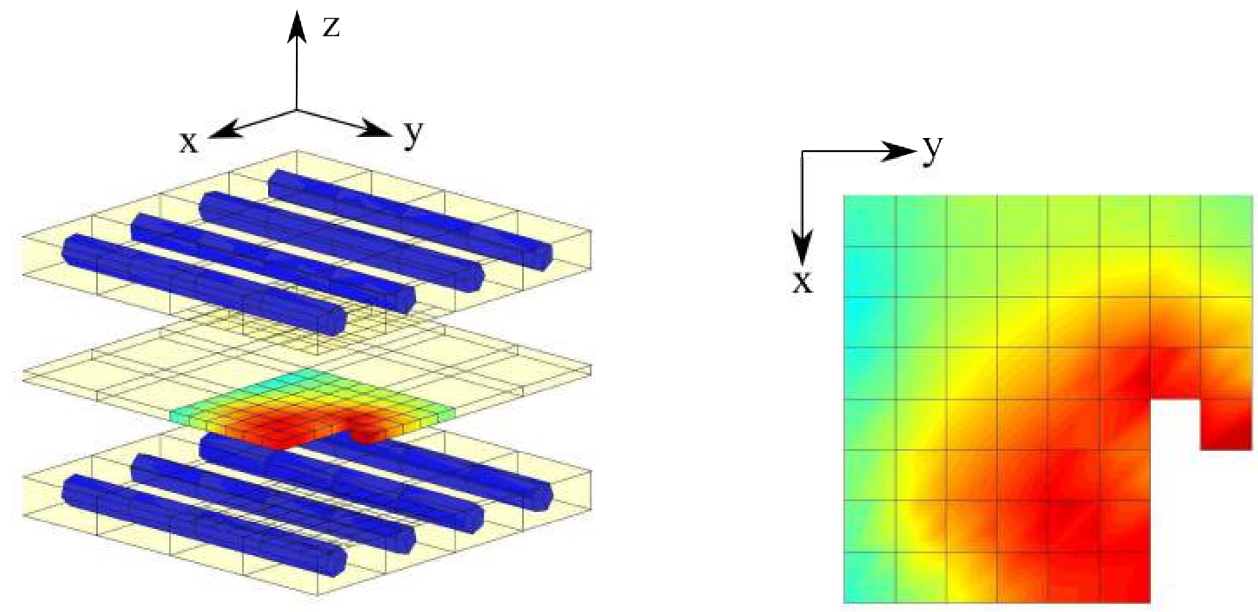

(b)
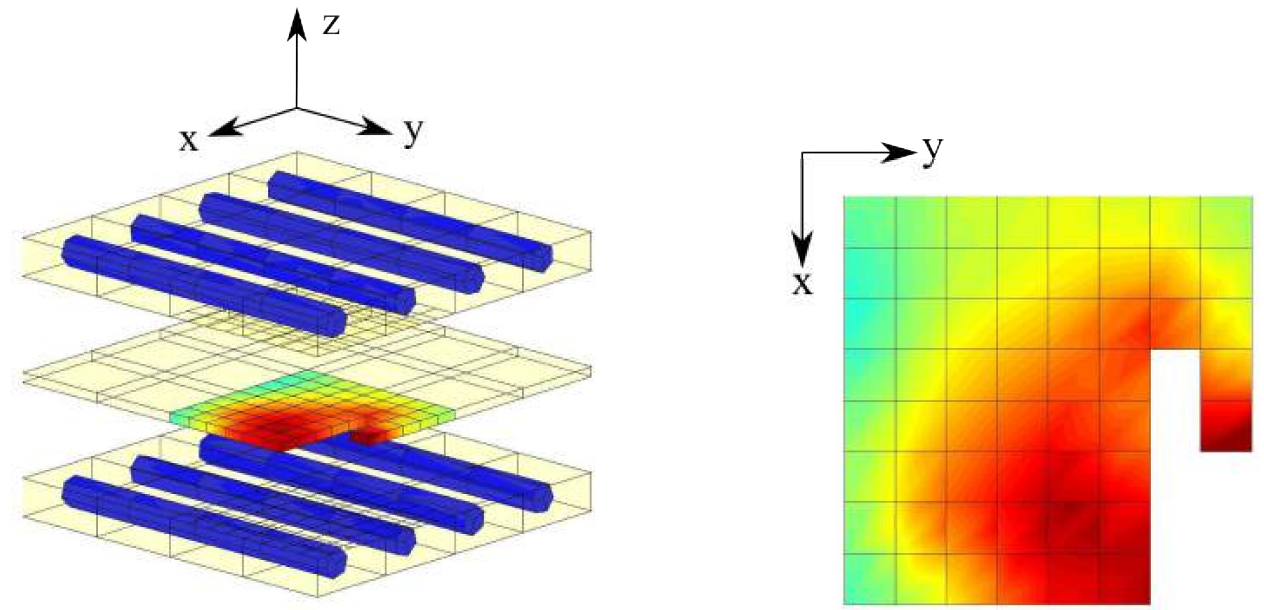

(c)

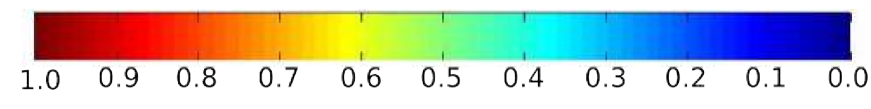

Figure 6.18: Multi-scale failure: (a) Time step 148, $P=5194 \mathrm{MPa}, t=73.5 \mathrm{~ms}$; (b) Time step $164, P=5607 \mathrm{MPa}, t=81.5 \mathrm{~ms}$; (c) Time step 180, $P=6019 \mathrm{MPa}, t=89.5 \mathrm{~ms}$ 


\subsubsection{Example B: Fiber composite model}

In this example the multi-scale failure criterion is tested for a fiber composite. The composite is loaded in the $z$ coordinate direction, as shown in Figure 6.19(b), boundary conditions are also shown in the figure. An initial failure is also assumed in this example. It is set at the corner of the interface layer of the composite, as shown in Figure 6.19(b). An initial load, $P_{0}$, of $3400 \mathrm{MPa}$ and a final load, $P_{\max }$, of $8500 \mathrm{MPa}$ are considered. The load cycle is completed in 100 time steps of $\Delta t=5 \times 10^{-4} \mathrm{~s}$. The materials for the matrix and the fibers are the same as in the previous example, namely, EPN-BPA epoxy for the matrix and Carbon fiber AS4 for the fibers.

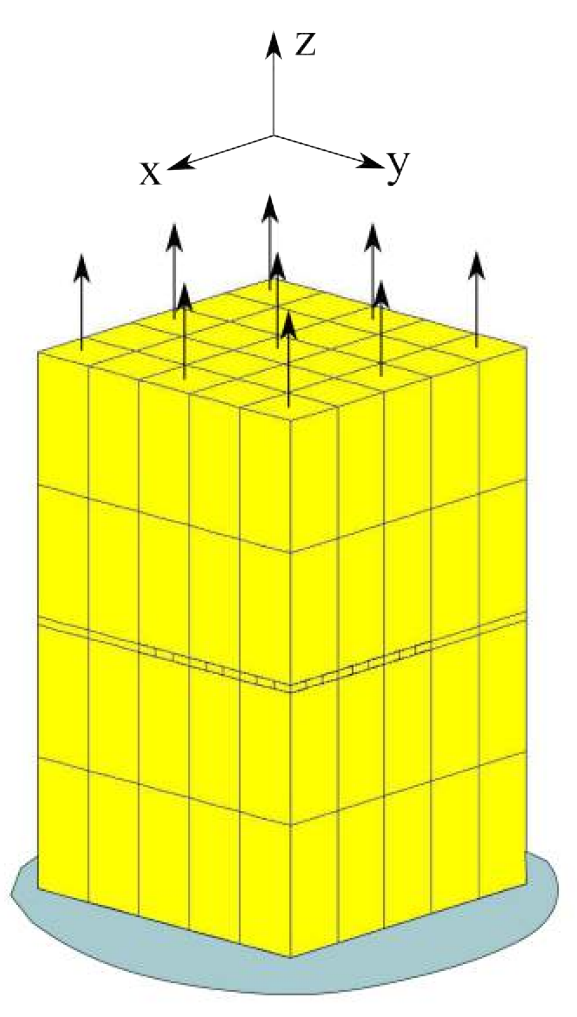

(a)

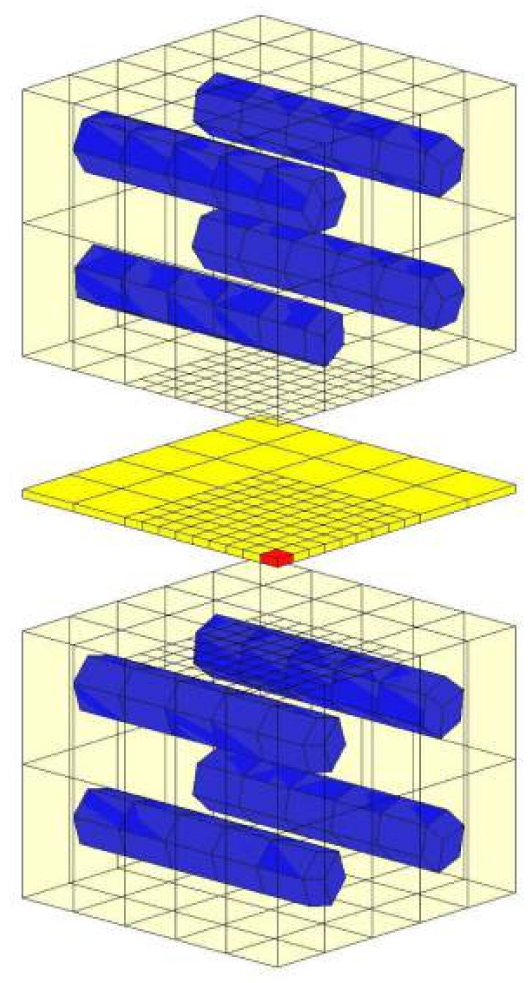

(b)

Figure 6.19: Short-fiber composite example; (a) Boundary conditions, (b) Fiber discretization and initial failure (red).

The composite was modelled with 1342 quadratic discontinuous elements, 10736 quadratic discontinuous nodes, resulting in 32208 DOFs. A total of 94 regions were created, from which, 81 regions were used for the multi-scale approach application. 
The ACA was applied for the generation of the BEM matrices. The ACA error tolerance is set to $\epsilon_{c}=10^{-4}$. The maximum number of elements per cluster was set to 20 and the admissibility parameter, $\eta$, to 0.9 . A total of 101 clusters and 1092 blocks were created, from which 286 were admissible pairs. The ACA was applied to three regions: The bottom matrix, the top matrix and the non-discretized area of the composite interface. For this example, the conventional BEM generated all matrices in $8027 \mathrm{~s}$, while the ACA generated them in $5775 \mathrm{~s}$, representing $28.1 \%$ gain in processing time. The total analysis time was approximately 8 hours. This example was performed on the Bigmem node from the Kahuna cluster that belongs to the CEPID project funded by FAPESP. The Bigmem node has 64 cores and 1TB RAM memory. It was requested 20 cores for the present example. The OpenMP interface was used for multi-processing among threads.

Figures 6.20, 6.21 and 6.22 show the multi-scale criterion applied to a pre-set discretized area within the composite for different time steps. The value for the criterion goes from 0 (blue) to 1 (red), the latter representing failure. Figure 6.20 (a-c) show the multi-scale criterion for time steps 1, 25 and 50, respectively. Figure 6.21 (a-c) show the multi-scale criterion for time steps 70, 71 and 72, respectively. Figure 6.22 (a-c) show the multi-scale criterion for time steps 73,74 and 75 , respectively.

In this example the failure tended to propagate in the in-plane radial direction. This failure propagation path is expected due to the symmetry of the geometry and load conditions. The Fast BEM approach resulted in a reduction of $28.1 \%$ in processing time, being an acceptable reduction time for the size of the problem. The mesh transition among elements is smoother than in the previous example. However, it was perhaps also necessary to conduct a mesh refinement study to ensure the convergence of the results. 

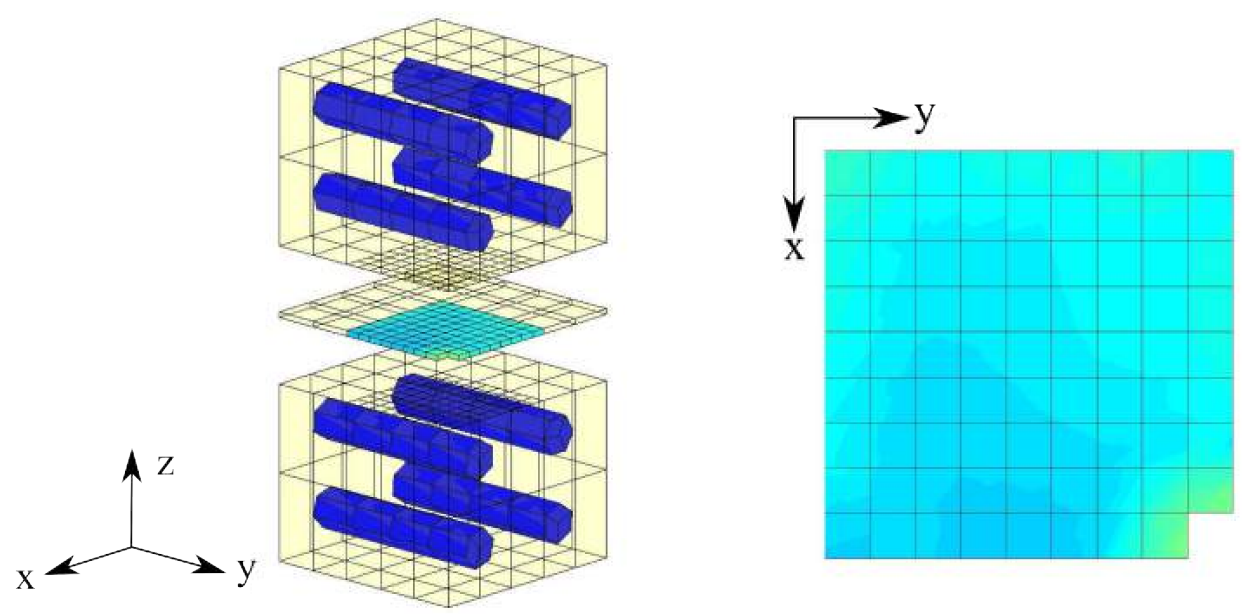

(a)
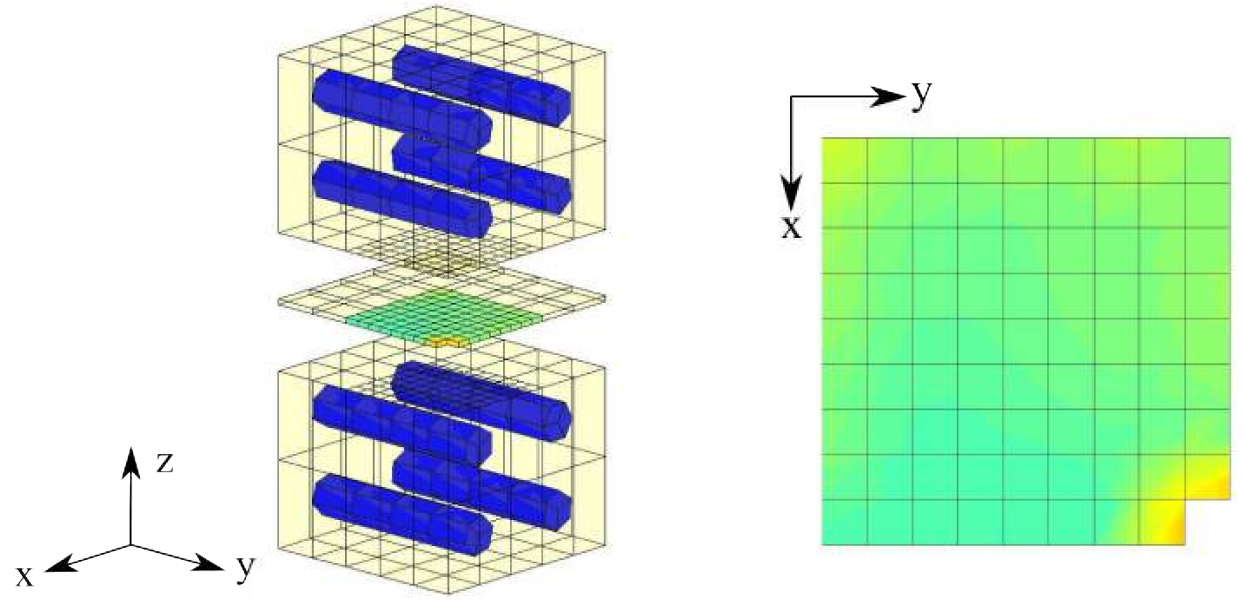

(b)
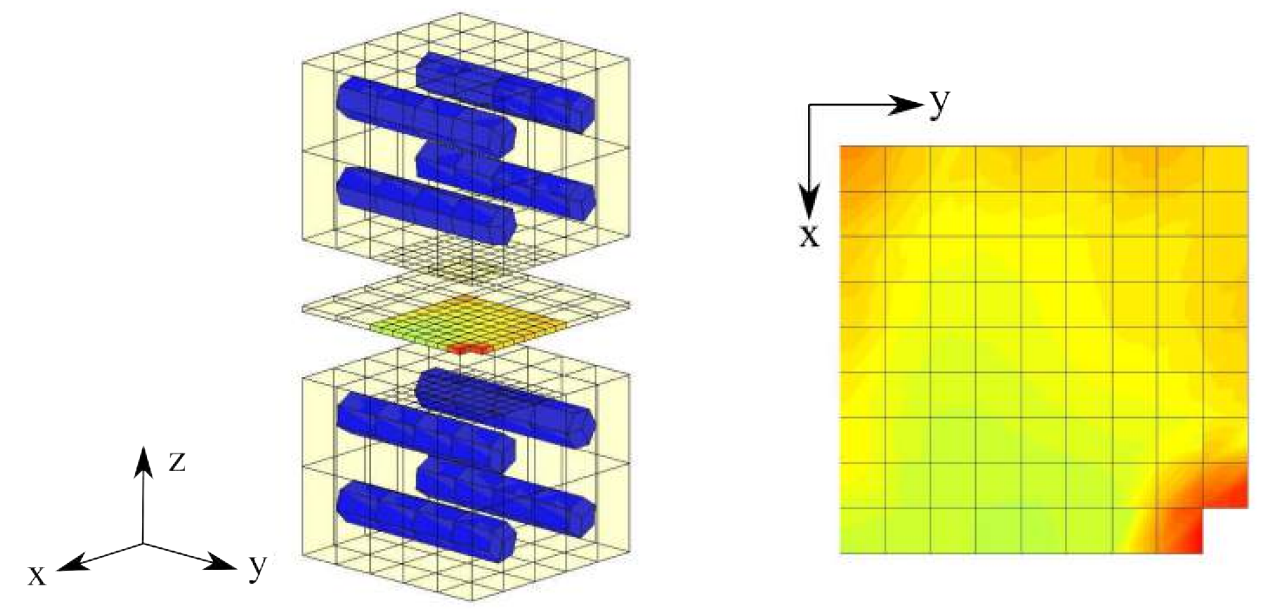

(c)

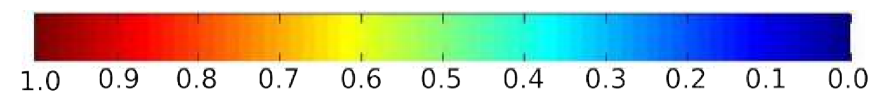

Figure 6.20: Multi-scale failure: (a) Time step 1, $P=3400 \mathrm{MPa}, t=0 \mathrm{~ms}$; (b) Time step 25, $P=4636 \mathrm{MPa}, t=12 \mathrm{~ms}$; (c) Time step 50, $P=5924 \mathrm{MPa}, t=24.5 \mathrm{~ms}$ 

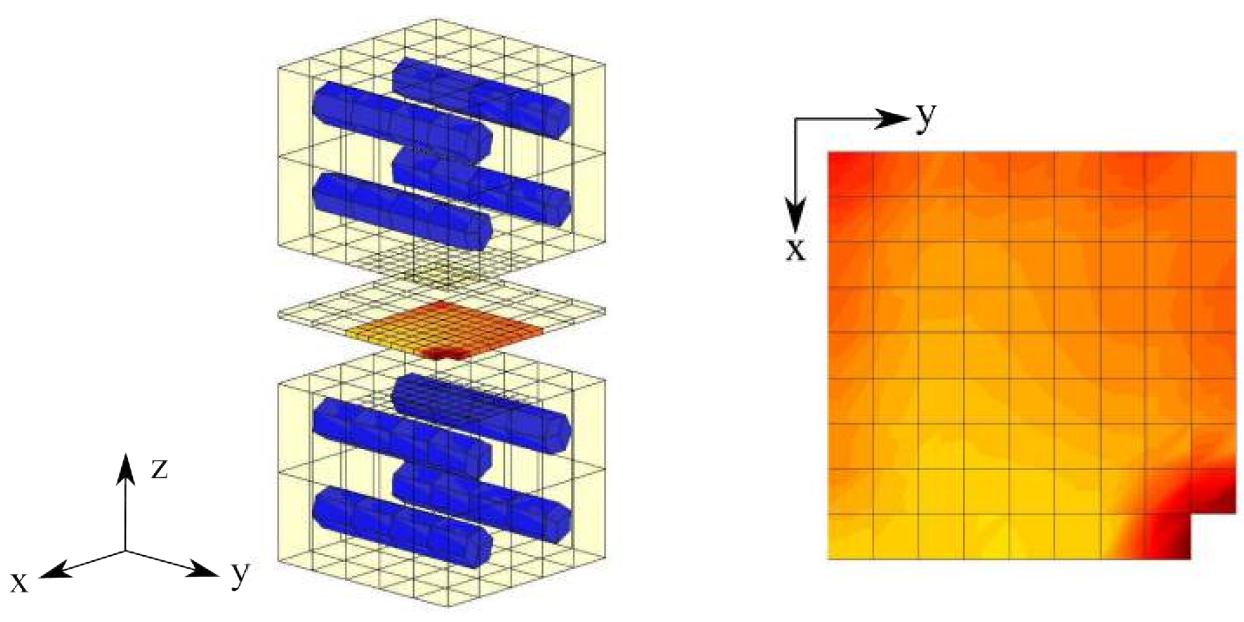

(a)
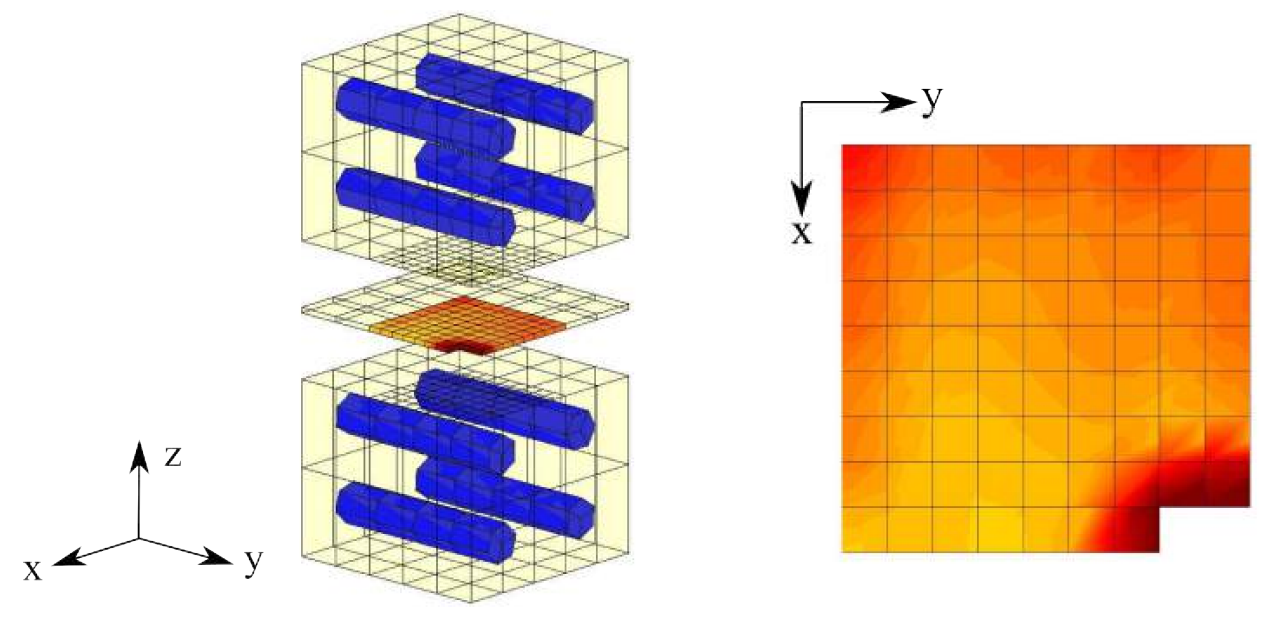

(b)

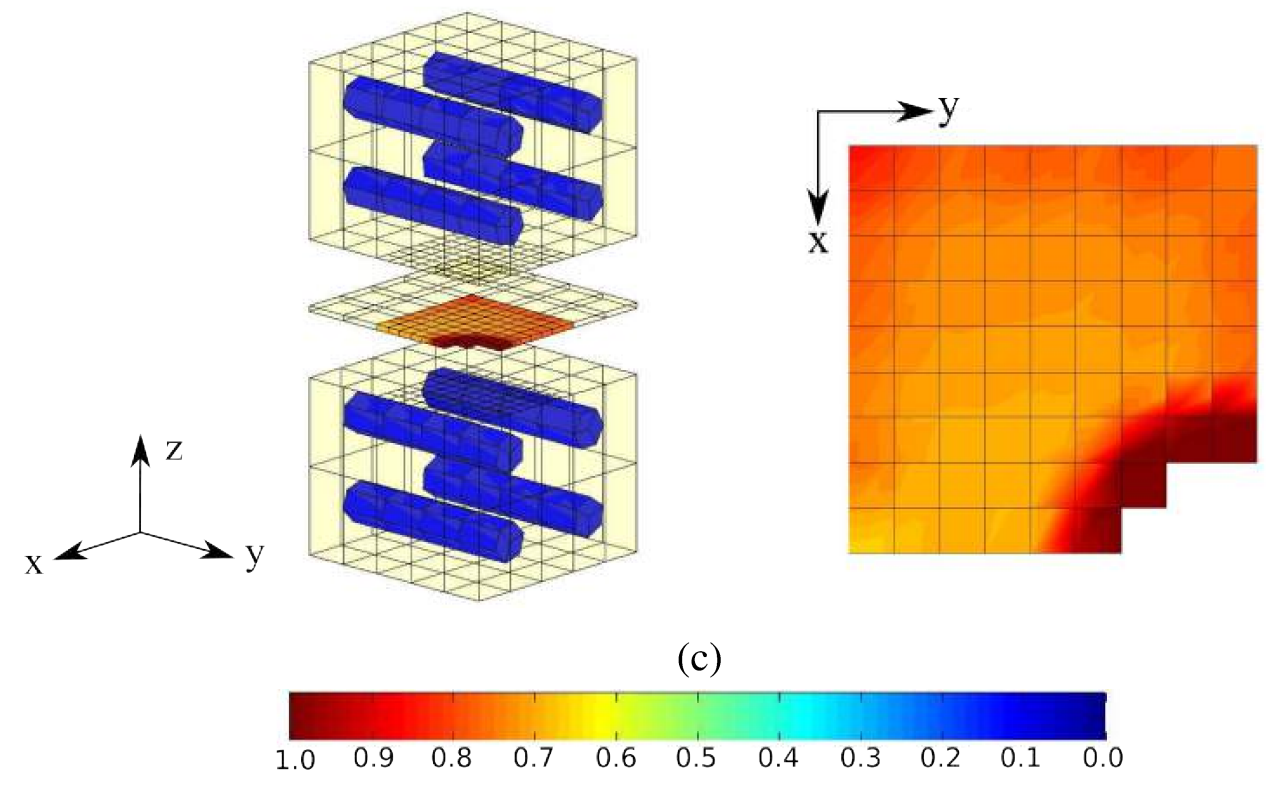

Figure 6.21: Multi-scale failure: (a) Time step 70, $P=6954 \mathrm{MPa}, t=34.5 \mathrm{~ms}$; (b) Time step $71, P=7006 \mathrm{MPa}, t=35 \mathrm{~ms}$; (c) Time step 72, $P=7057 \mathrm{MPa}, t=35.5 \mathrm{~ms}$ 

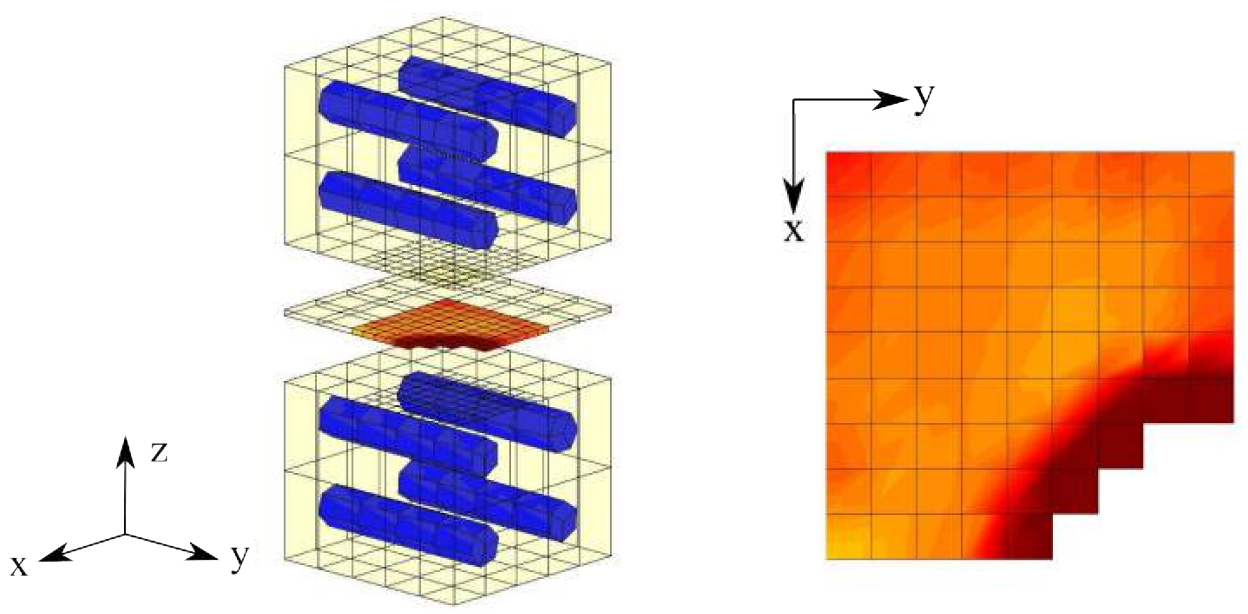

(a)
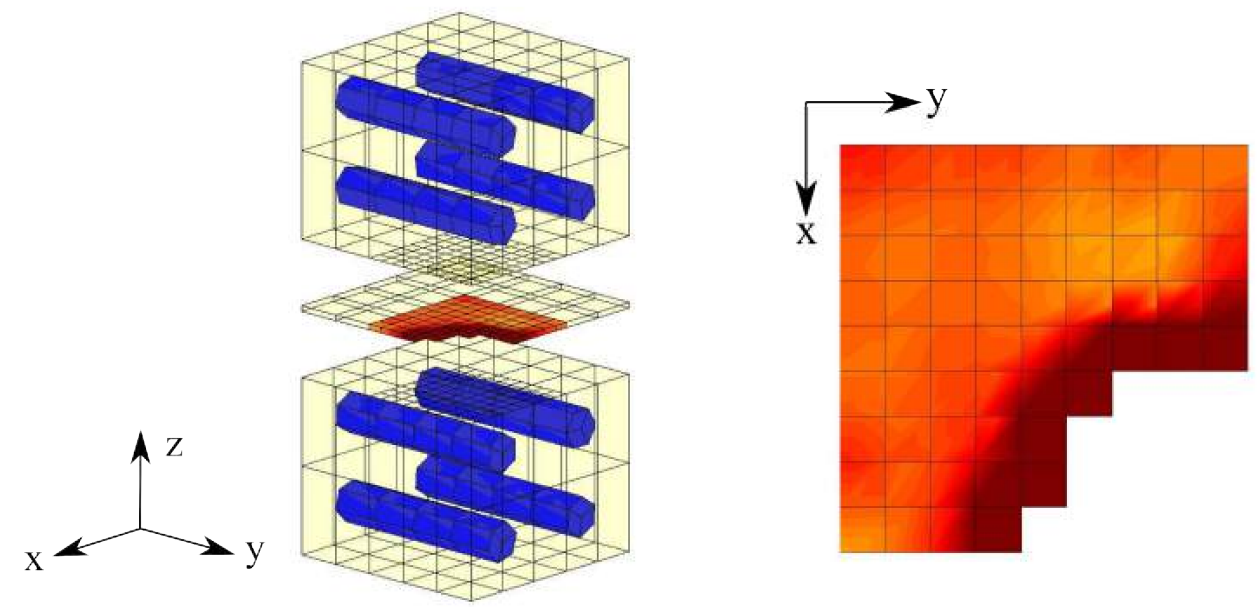

(b)
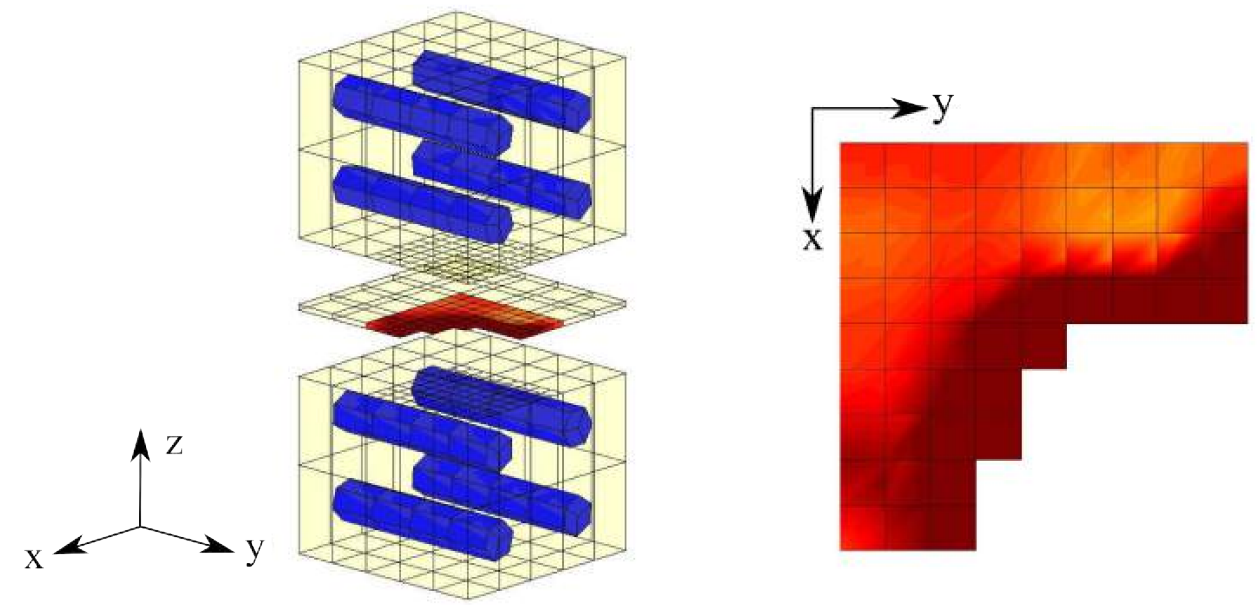

(c)

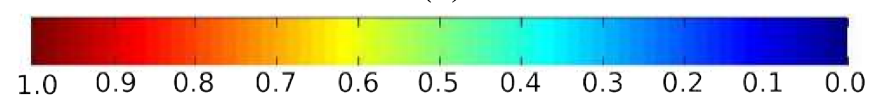

Figure 6.22: Multi-scale failure: (a) Time step 73, $P=7109 \mathrm{MPa}, t=36 \mathrm{~ms}$; (b) Time step 74, $P=7160 \mathrm{MPa}, t=36.5 \mathrm{~ms}$; (c) Time step 75, $P=7212 \mathrm{MPa}, t=37 \mathrm{~ms}$ 


\subsubsection{Example C: Laminate Composite with a central flaw}

In this example the multi-scale failure criterion is tested for a laminate composite with a $0^{\circ} / 0^{\circ}$ fiber distribution and containing a central defect. The laminate is loaded in the $x$ coordinate. Boundary conditions are shown in Figure 6.23(a). An initial failure, by considering material absence, is set at the center of the interface of the composite, as can be seen in Figure 6.23(b). An initial load, $P_{0}$, of $8100 \mathrm{MPa}$ and final load, $P_{\max }$, of $13500 \mathrm{MPa}$ are considered. The load process is completed in 100 time steps of $\Delta t=5 \times 10^{-4} \mathrm{~s}$. The materials for the matrix and the fibers are the same as in the previous examples.

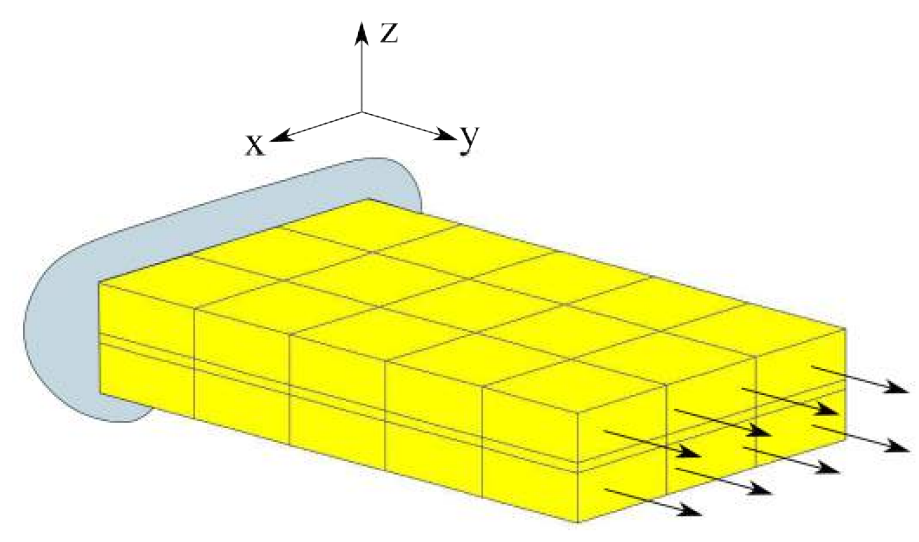

(a)

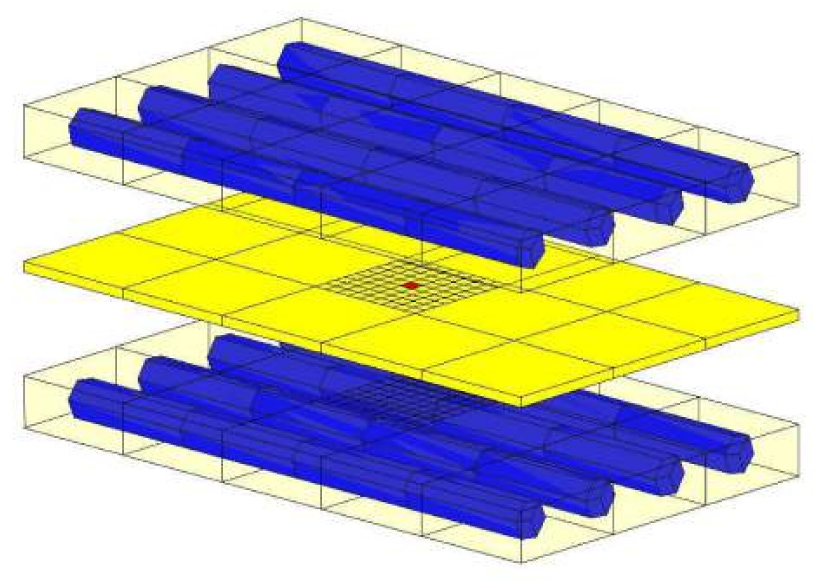

(b)

Figure 6.23: Laminate composite with a $0^{\circ} / 0^{\circ}$ fiber distribution; (a) Boundary conditions, (b) Fiber discretization and initial failure (red).

The composite was modelled with 1298 quadratic discontinuous elements, 10384 quadratic discontinuous nodes, resulting in 31152 DOFs. A total of 91 regions were created, 
from which, 81 regions were used for the multi-scale approach application.

The ACA was applied for the generation of the BEM matrices. The ACA error tolerance is set to $\epsilon_{c}=10^{-4}$. The maximum number of elements per cluster was set to 20 and the admissibility parameter, $\eta$, to 0.9 . A total of 109 clusters and 1072 blocks were created, from which 552 were admissible pairs. The ACA was applied to three regions: The bottom matrix, the top matrix and the non-discretized area of the composite interface. For this example, the conventional BEM generated all matrices in 5223s, while the ACA generated them in 4682s, representing only $10.4 \%$ gain in processing time. The total analysis time was approximately 5 hours and 30 minutes. This example, as before, was performed on the Bigmem node from the Kahuna cluster. It requested 14 cores for the present example. The OpenMP interface was used for multi-processing among threads.

Figures 6.24, 6.25 and 6.26 show the multi-scale criterion applied to a pre-set discretized area within the composite for different time steps. The value for the criterion goes from 0 (blue) to 1 (red), the latter representing failure. Figure 6.24 (a-c) show the multi-scale criterion for time steps 1, 25 and 50, respectively; Figure 6.25 (a-c) show the multi-scale criterion for time steps 58, 59 and 60, respectively; and Figure 6.26 (a-c) show the multi-scale criterion for time steps 61,62 and 63 , respectively.

In this example the failure tended to propagate in the fiber direction, $y$ coordinate axis. This failure propagation path is somewhat expected, as the stresses in the matrix of the fiber reinforced laminae will be lower when compared with those in the cohesive layer; the fibers will carry most of the load applied in the same direction of the fiber alignment. The Fast BEM approach resulted in a reduction of $10.4 \%$ in matrix generation processing time. This low reduction is mainly due the more irregular geometry, as the same ACA parameters of the previous examples were used. A more in-depth study of the ACA parameters is necessary to achieve the best reduction in cpu time; likewise of the choice of the mesh design to ensure convergence of the results. For the present purpose, it has been clearly shown here that a numerical tool using the fast BEM approach of ACA has been successfully developed for the multi-scale failure analysis of anisotropic laminates. 

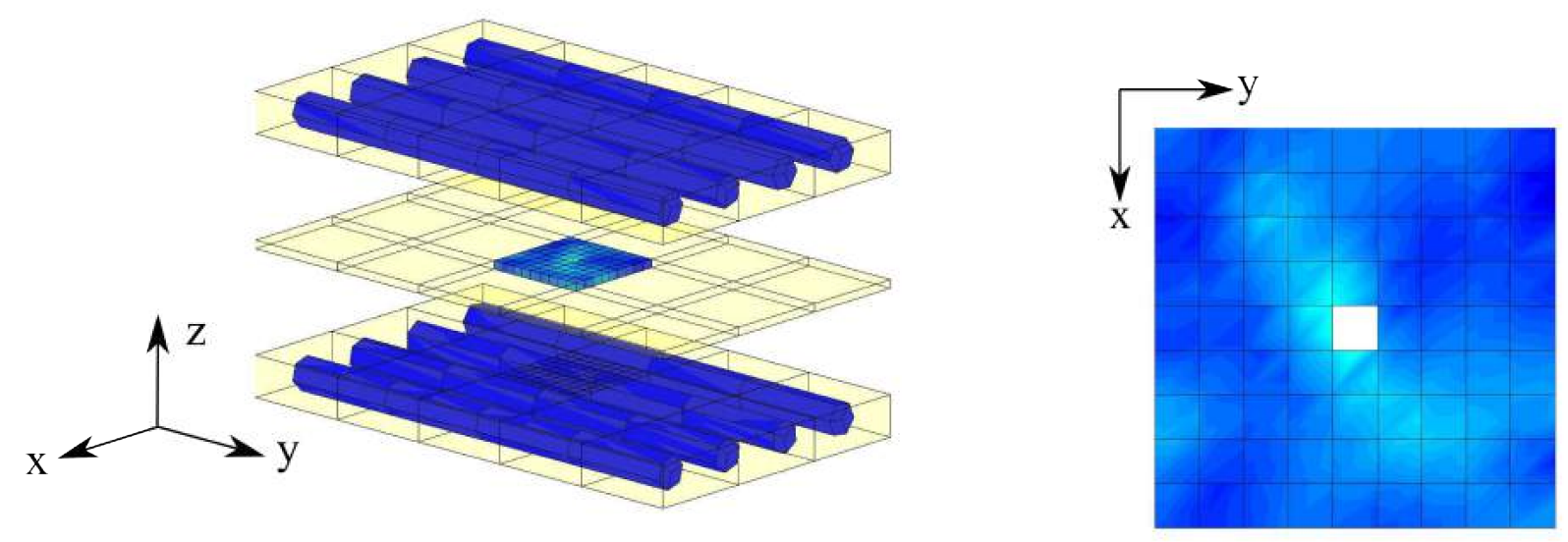

(a)
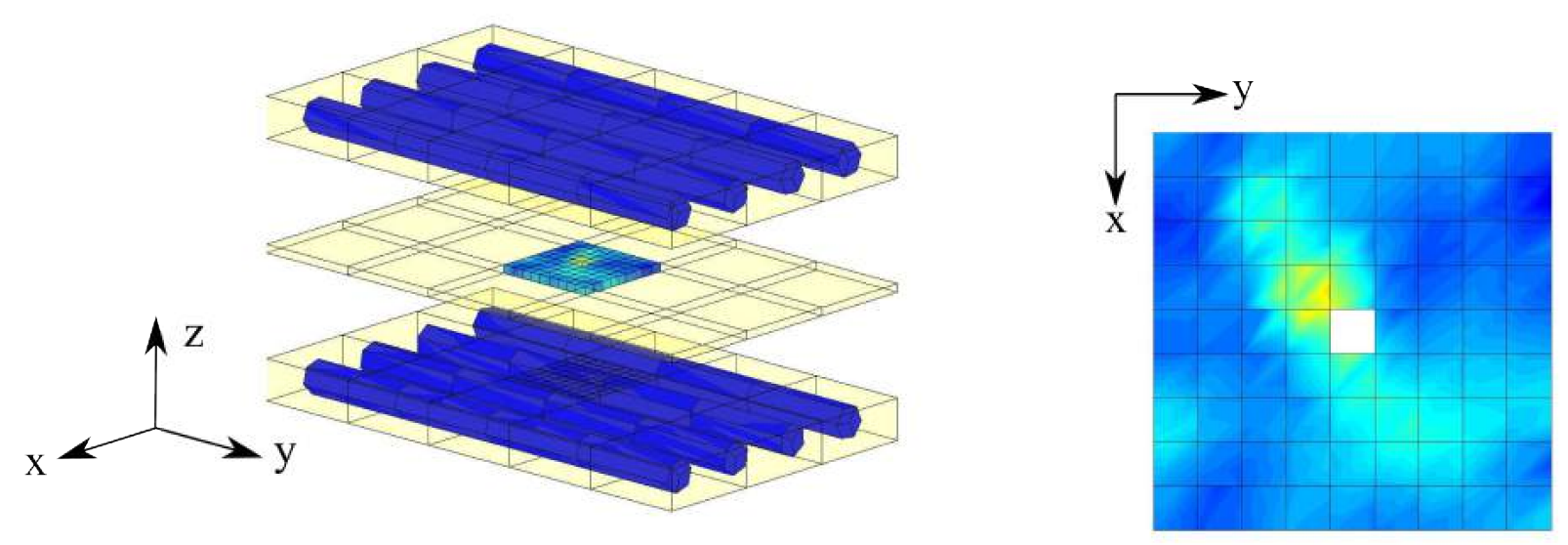

(b)
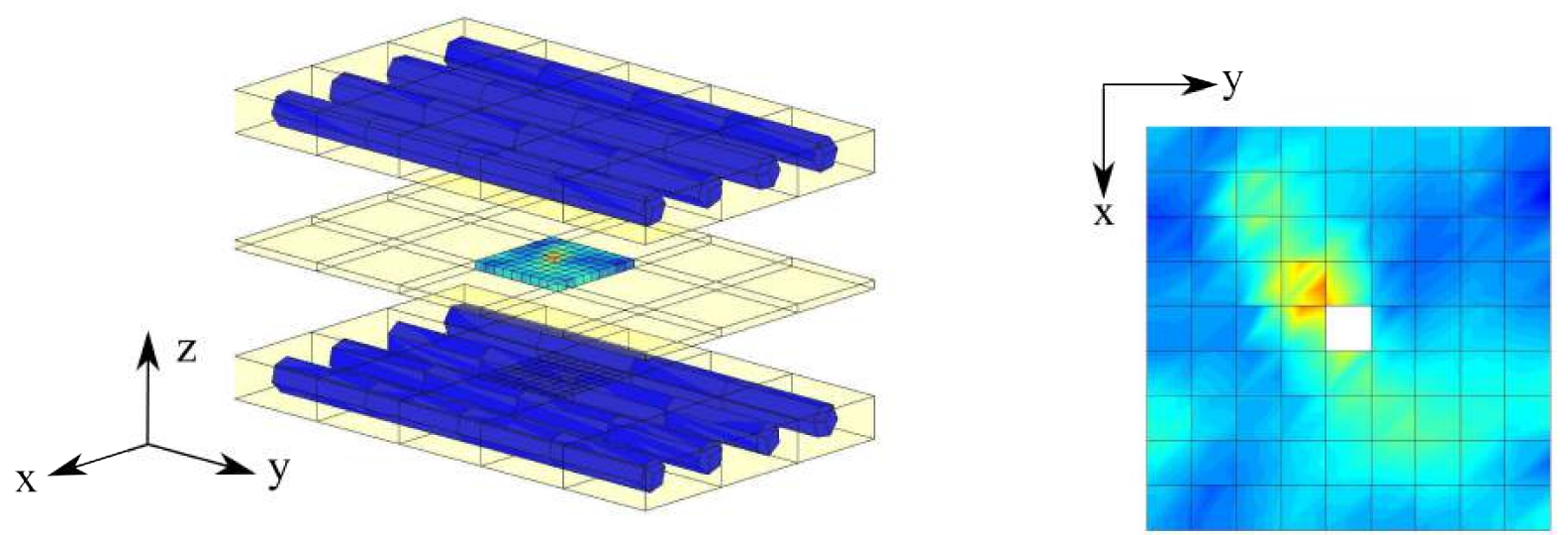

(c)

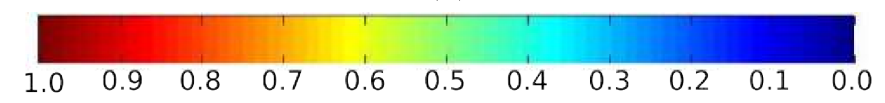

Figure 6.24: Multi-scale failure: (a) Time step 1, $P=8100 \mathrm{MPa}, t=0 \mathrm{~ms}$; (b) Time step 25, $P=9409 \mathrm{MPa}, t=12 \mathrm{~ms}$; (c) Time step 50, $P=10773 \mathrm{MPa}, t=24.5 \mathrm{~ms}$ 

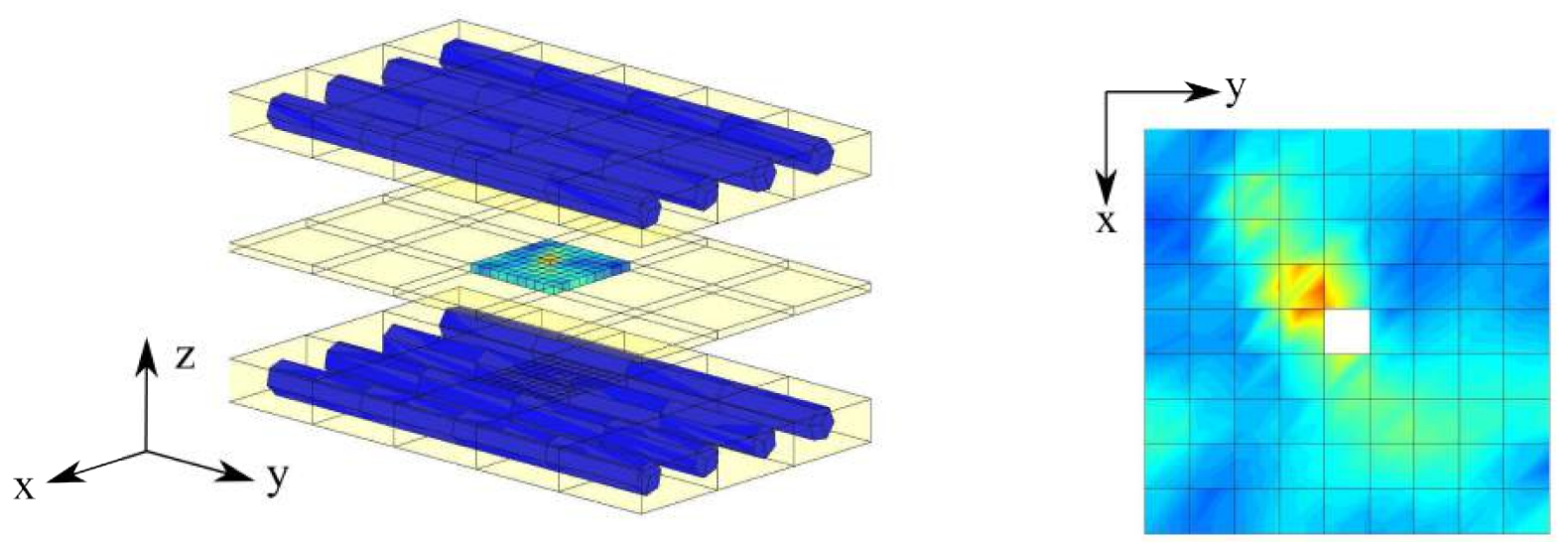

(a)
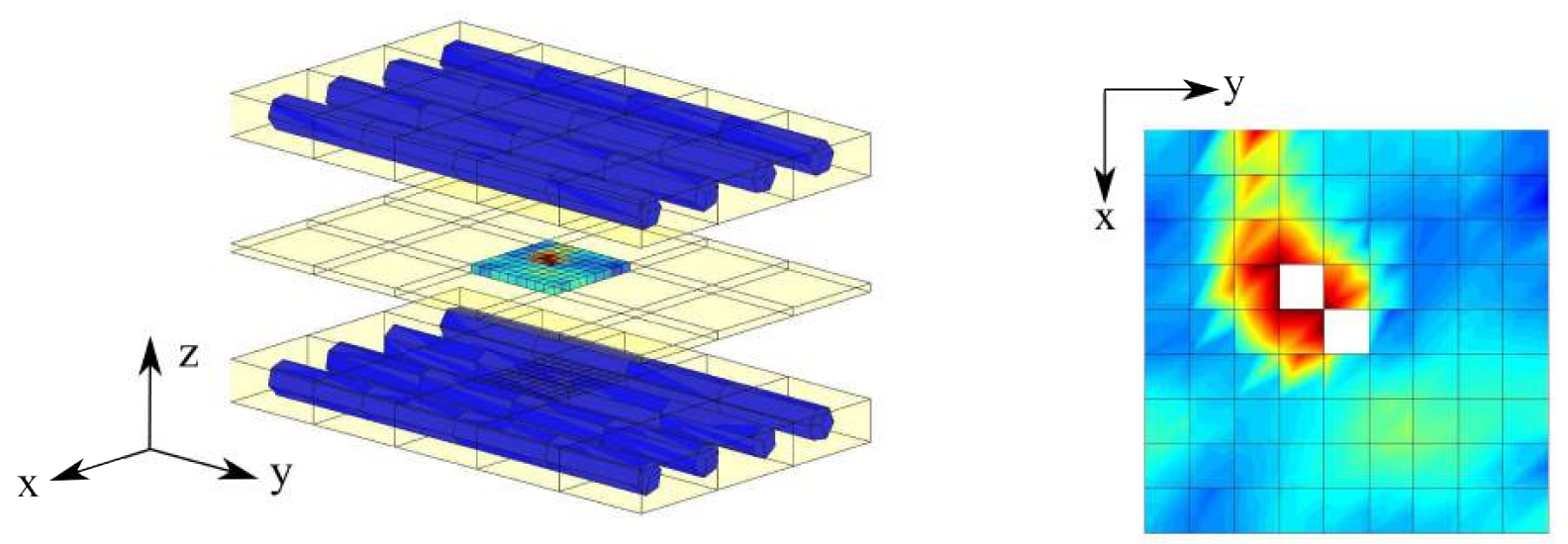

(b)
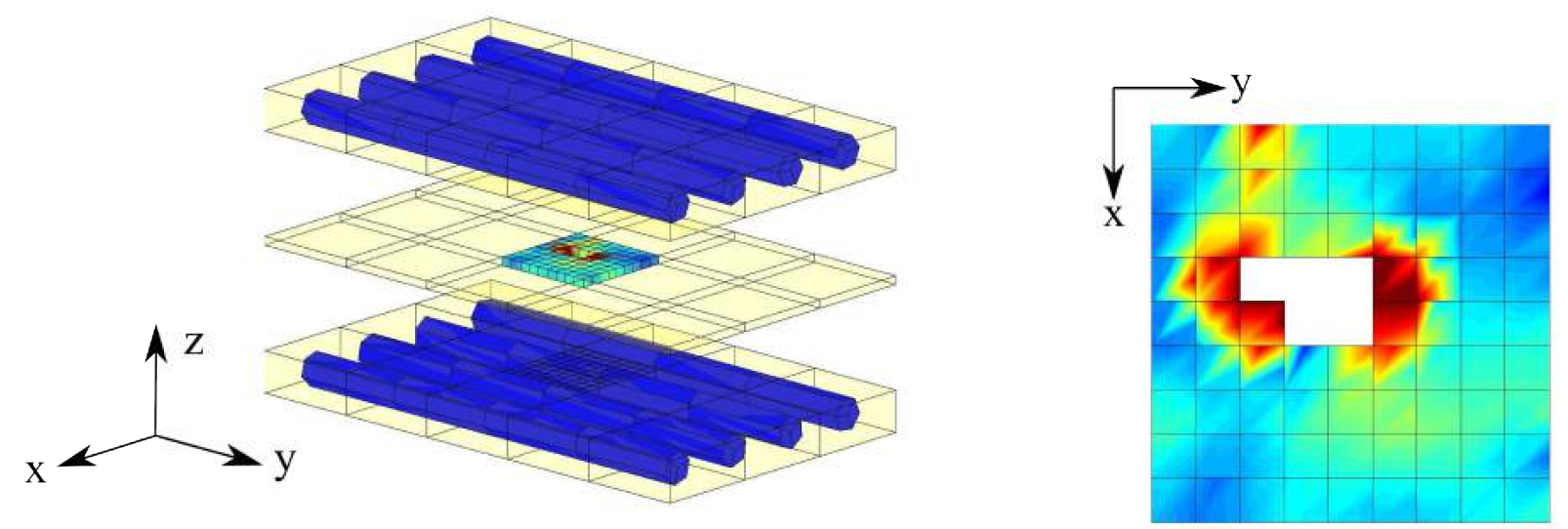

(c)

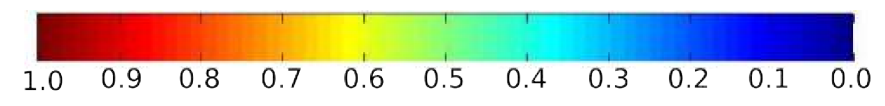

Figure 6.25: Multi-scale failure: (a) Time step 58, $P=11209 \mathrm{MPa}, t=28.5 \mathrm{~ms}$; (b) Time step 59, $P=11264 \mathrm{MPa}, t=29 \mathrm{~ms}$; (c) Time step 60, $P=11318 \mathrm{MPa}, t=29.5 \mathrm{~ms}$ 

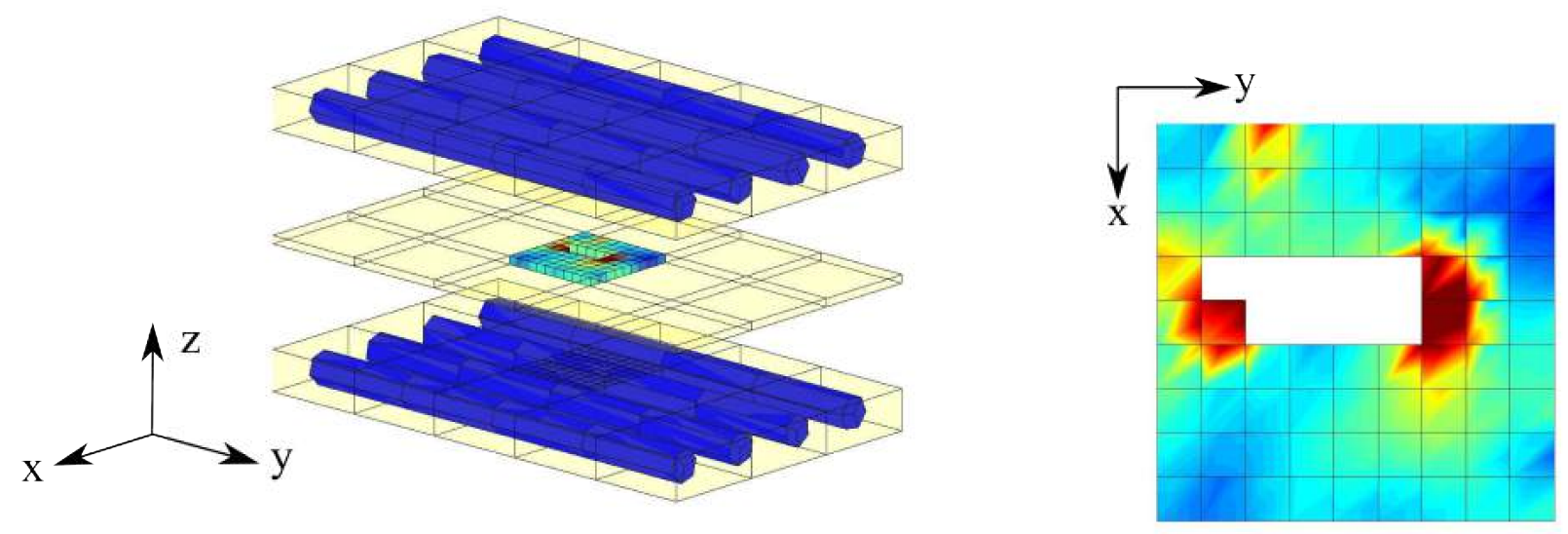

(a)
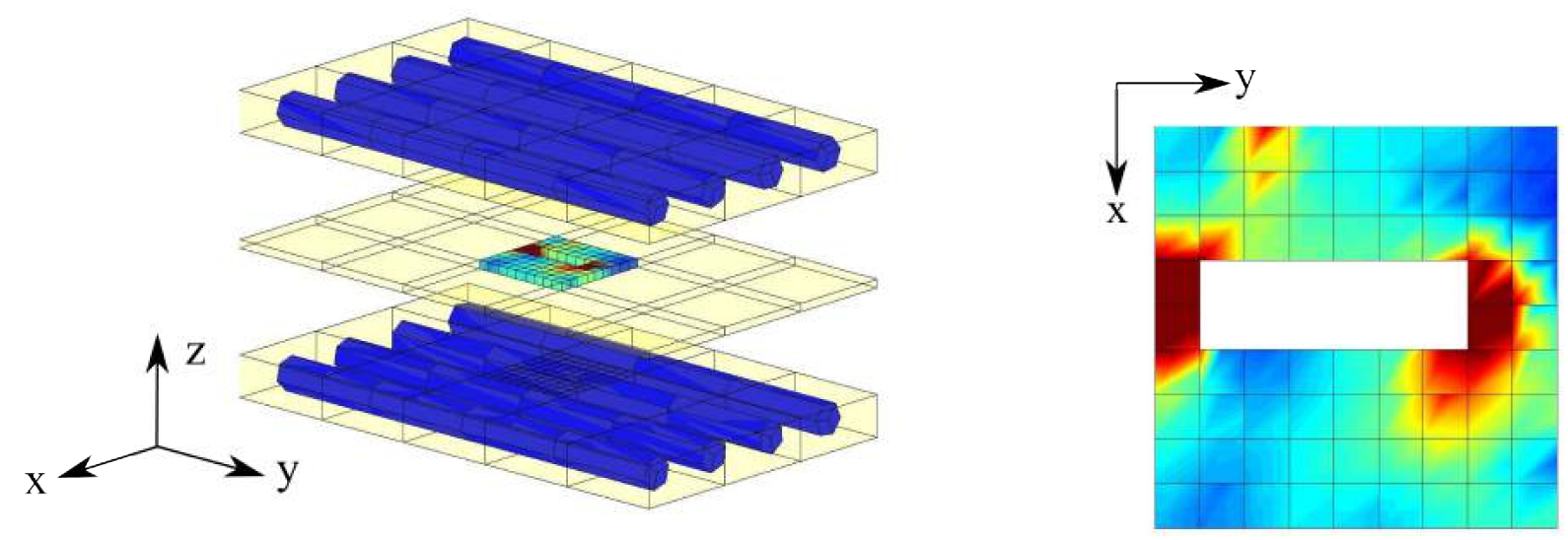

(b)
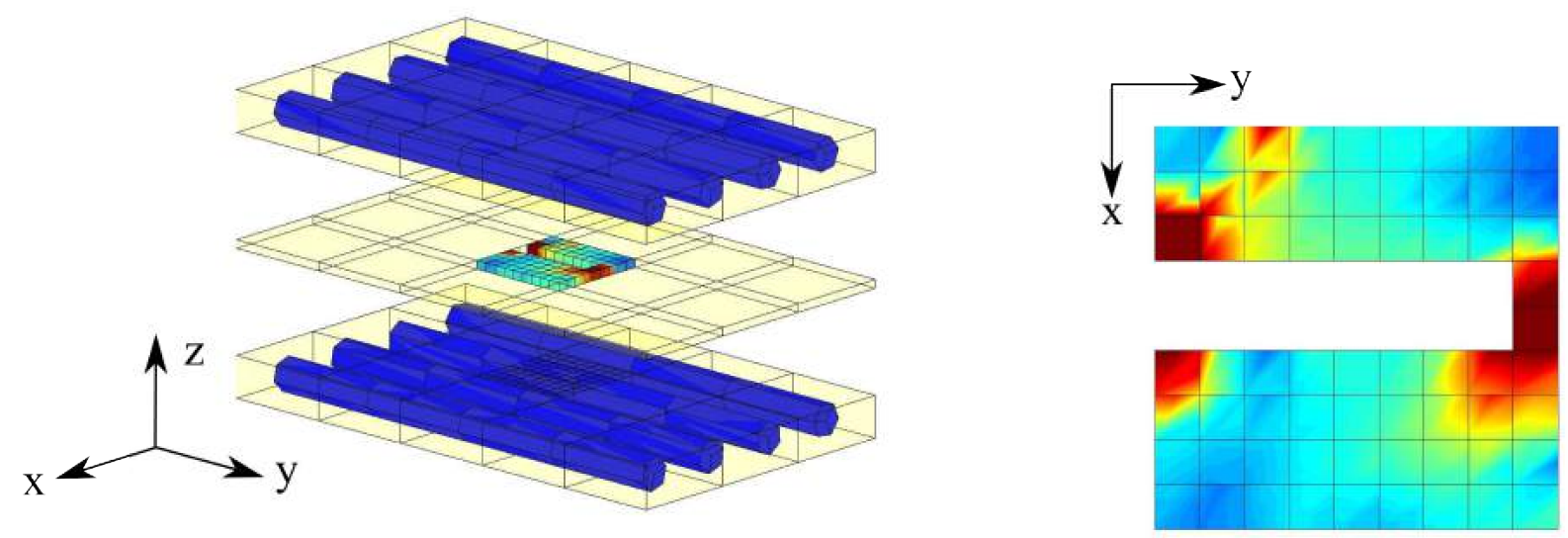

(c)

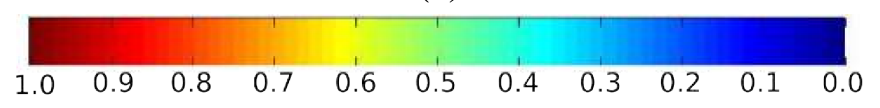

Figure 6.26: Multi-scale failure: (a) Time step 61, $P=11373 \mathrm{MPa}, t=30 \mathrm{~ms}$; (b) Time step 62, $P=11427 \mathrm{MPa}, t=30.5 \mathrm{~ms}$; (c) Time step 63, $P=11482 \mathrm{MPa}, t=31 \mathrm{~ms}$ 


\section{Concluding Remarks}

\subsection{Final conclusions}

Some particular conclusions have been already presented in the numerical result sections of the respective chapters. As general conclusions, the following may be cited,

A state-of-the art of multi-scale analysis of composites using BEM and acceleration methods has been presented in the study. The constitutive and equilibrium equations for anisotropic materials have been briefly reviewed in the first two chapters. This is with the intention of providing a basic background for the understanding of the subsequent chapters.

The 3D fundamental solution for generally anisotropic solids has been implemented according to the theory developed by (TAN et al., 2013). Taking advantage of the periodic nature of Green's function when expressed in spherical coordinates, they can be represented by double Fourier series. The most significant advantage of using this Fourier series representation of the Green's function and its derivatives is that the Fourier coefficients need to be evaluated just once for a given material. This reduced the computational efforts, besides the relatively simplicity of the implementation into a BEM code. In the BEM analysis, the laminate composite model was constructed using the multi-domain method, due to the necessity of modeling different types of material at the same time.

A fast BEM approach based on hierarchical clustering and low rank approximations, by means of the Adaptive Cross Approximation (ACA), has also been implemented. The fast BEM approach was first tested by successfully applying it to a $2 \mathrm{D}$ multiple inclusion potential $2 \mathrm{D}$ problem. The fast BEM approach has also been applied to 3D single-domain, multi-domain, short fibers-matrix models and composite laminates. A significant reduction in CPU processing time was observed in all cases analysed, while maintaining the pre-set accuracy.

In BEM analysis of dynamic problems a domain integral exists in the primary formulation of the BIE. This domain integral needs to be transformed into surface ones. To this end, the Dual Reciprocity (DRM) and the Radial Integration Method (RIM) have been implemented in this study as well. This has allowed time-dependent problems to be analysed using the BEM, in which the integration through time was carried out using the Houbolt algorithm. In general, it was observed that, small time intervals lead to a more accurate dynamic response, but they increase the risk of instability. The RIM was found to be more accurate and stable than the 
DRM, even with a relatively coarser mesh and smaller time steps. However, it demands high computational requirements leading to prohibitive times when analysing problems with high number of DOFs. Hence, the DRM was used in the dynamic failure multi-scale model.

A multi-scale failure criterion has also been implemented and applied to simple laminate and fiber composites. Failure was considered to occur at the interface in all cases treated. For the micro-scale analysis, a recent theory on potential between molecules was used, (YANG et al., 2014). This potential is applicable to epoxy, used as the matrix material in composites. The Cauchy-Born rule has been used to couple the scales on the 3D multi-scale model. The failure progression depends on several material and geometry parameters, as well as, the boundary conditions. Failure progression was observed to occur at sites where high shear stresses and deformation gradients were present.

The numerical implementation of the methods and models which were the foundation of the present thesis are not exact, and are subjected to the usual numerical approximations. They include: the Double Fourier representation of the fundamental solutions; numerical integration of the Fourier coefficients; low rank approximation of admissible blocks within the Fast BEM approach; approximation of continuous models by the multi-domain approach; numerical transformation of the domain integrals in the BIE; epoxy novel potential for the multi-scale approach implementation based on Molecular Dynamics and experimental data; numerical differentiation and integration of the potential. One other limitation is directly related with the size of the problem that is possible to analyse. Computational resources were one of the main constraints for the analysis of bigger and more realistic problems to be carried out.

In this study, the computational code was implemented in Fortran 90, using the GNU com-

piler gfortran. The multiprocessor parallelization of some parts of the code was accomplished using the OpenMP interface. The visualization was obtained using MATLAB. Moreover, the National Center for High Performance Computing in São Paulo (CENAPAD) and the Centros de Pesquisa, Inovação e Difusão (CEPID) provided the conditions to test the multi-scale problems herewith presented.

\subsection{Suggestions for further research}

The present work has been a successful attempt to implement an integrated approach of carrying out multi-scale analysis of composites failure, as summarized above. It can be further improved upon and extended in several respects, some of which are as follows: 
(a) Inclusion of thermoelastic effects in the model. A new formulation has been recently proposed by (SHIAH AND TAN, 2014), in which the generally anisotropic fundamental solution based on Fourier series was used to deal with the domain integral without the need of using an approximation method such as the DRM or the RIM. This formulation is based on exact analytical domain-to-boundary integral transformation and lead also to more accurate and efficient algorithms.

(b) Optimize the RIM to achieve the same efficiency as DRM for the same accuracy, so this scheme continues to be employed.

(c) Investigate more general theories for the atomic/molecular potential applicable to polymers. The lack of material properties makes the model dependent on Molecular Dynamics (MD) results. A more in-depth study should be make to this end.

(d) Consider the use of Message Passing Interface (MPI) to permit the parallelization among a big number of computer nodes and do not be restricted to a single node. This will allow larger multi-scale models to be treated.

(e) At the macro-scale, laminates are thin-bodies, as engineering structures or components. Thin-body problems present some challenges of near singularity of the integral equation in BEM formulations. Implementation of algorithms that can resolve those issues will allow the multi-scale analysis of even larger, practical problems. 


\section{References}

ABOUDI, J. Micromechanical analysis of composites by the method of cells. Appl. Mech. Rev., v. 42, 193-221, 1989.

ABOUDI, J. The generalized method of cells and high-fidelity generalized method of cells micromechanical model - a review. Mechanics of Advanced Materials and Structures, v. 11, n. 4-5, 329-366, 2004.

URL: http://dx.doi.org/10.1080/15376490490451543

AGNANTIARIS, J.P.; POLYZOS, D. and BESKOS, D.E. Some studies on dual reciprocity BEM for elastodynamic analysis. Computational Mechanics, v. 17, 270 - 277, 1996.

ALBUQUERQUE, E. and SOLLERO, P. The boudary element method applied to transient dynamic anisotropic problems. In A. Kassab; C. Brebbia and M. Chopra, editors, Proc. Boundary Element Method XX, pp. 617-624. Orlando - USA, 1998.

ALBUQUERQUE, E.; SOlLERO, P. and ALIABADI, M. Computation of dynamic stress intensity factor in anisotopic materials using the boundary element method. In P. Pimenta; R. Brasil and E. Almeida, editors, Proc. XX Cilamce. Sao Paulo - Brazil, 1999a.

ALBUQUERQUE, E.; SOLLERO, P. and ALIABADI, M. The dual boundary element formulation applied to dynamic fracture mechanics in anisotropic materials. In M. Aliabadi, editor, Proc. Boundary Element Techniques, pp. 23-29. London - UK, 1999 b.

ALBUQUERQUE, E.; SOLLERO, P. and ALIABADI, M. The boundary element method applied to time dependent problems in anisotropic materials. International Journal of Solids and Structures, v. 39, n. 5, 1405-1422, 2002.

ALBUQUERQUE, E.; SOLLERO, P. and ALIABADI, M. Dual boundary element method for 
anisotropic dynamic fracture mechanics. International Journal for Numerical Methods in Engineering, v. 59, 1187-1205, 2004.

ALBUQUERQUE, E.; SOLLERO, P. and FEDELINSKI, P. Analysis of anisotropic dynamic crack problems using the dual reciprocity boundary element method in Laplace domain. In Proc. ECCM. Krakow - Poland, 2001.

ALBUQUERQUE, E.; SOLLERO, P. and FEDELINSKI, P. Dual reciprocity boundary element method in Laplace domain applied to anisotropic dynamic crack problems. Computers and Structures, v. 81, 1703-1713, 2003a.

ALBUQUERQUE, E.; SOLLERO, P. and FEDELINSKI, P. Free vibration analysis of anisotropic material structures using the boundary element method. Engineering Analysis with Boundary Elements, v. 27, 977-985, 2003b.

ALBUQUERQUE, E.; SOLLERO, P. and PORTILHO DE PAIVA, W. The radial integration method applied to dynamic problems of anisotropic plates. Communications in Numerical Methods in Engineering, v. 23, n. 1, 805-818, 2007a.

ALBUQUERQUE, E.; SOLLERO, P.; VENTURINI, W. and ALIABADI, M. Boundary element method analysis of anisotropic Kirchhoff plates. International Journal of Solids and Structures, v. 43, 4029-4046, 2006.

ALBUQUERQUE, E.L.; SOLLERO, P. and DE PAIVA, W.P. The BEM and the RIM in the dynamic analysis of symmetric laminate composite plates. In M. Alves and H.S. da Costa Mattos, editors, Mechanics of Solids in Brazil 2007. Brazilian Society of Mechanical Sciences and Engineering, Sao Paulo, Brazil, 2007b.

ALIABADI, M. The Boundary Element Method, vol2: Applications in Solids and Structures. John Wiley \& Sons, 2002.

ANDRIANOV, I.V.; DANISHEVS'KYY, V.V. and WEICHERT, D. Simple estimation on effective transport properties of a random composite material with cylindrical fibres. Z. angew. Math. Phys., v. 59, 889-903, 2008. 
ASHTON, J.E.; HALPIN, J.C. and PETIT, P.H. Primer on Composite Materials: Analysis. Technomic Publishing AG, 1968.

ATKINSON, K.E. The numerical evaluation of particular solutions for Poisson's equation. IMA Journal of Numerical Analysis, v. 5, 319 - 338, 1985.

BEBENDORF, M. Approximation of boundary element matrices. Numerishce Mathematik, v. $86,565-589,2000$.

BEBENDORF, M. and GRZHIBOVSKIS, R. Accelerating Galerkin BEM for linear elasticity using ACA. Mathematical Methods in the Applied Sciences, v. 29, 1721 - 1747, 2006.

BEBENDORF, M. and RJASANOW, S. Adaptive low-rank approximation of collocation matrices. Computing, v. 70, $1-24,2003$.

BELSKY, V.; BEALL, M.; FISH, J.; SHEPHARD, M. and GOMAA, S. Computer-aided multiscale modeling tools for composite materials and structures. Computing Systems in Engineering, v. 6, n. 3, $213-223,1995$.

URL: http://www.sciencedirect.com/science/article/pii/095605219500019V

BENEDETTI, I. and ALIABADI, M. A fast hierarchical dual boundary element method for three-dimensional elastodynamic crack problems. International Journal for Numerical Methods in Engineering, v. 84, n. 9, 1038-1067, 2010.

BENEDETTI, I.; ALIABADI, M. and DAVI, G. A fast 3D dual boundary element method based on hierarchical matrices. Solids and Structures, v. 45, 2355 - 2376, 2008.

BENEDETTI, I.; MILAZZO, A. and ALIABADI, M. A fast dual boundary element method for 3D anisotropic crack problems. International Journal for Numerical Methods in Engineering, v. 80, $1356-1378,2009$.

BENEDETTI, I.; MILAZZO, A. and ALIABADI, M. Fast hierarchical boundary element method for large-scale 3-D elastic problems. In: Boundary element methods in Engineering and Sciences, vol 4. Imperial College Press, 2011. 
BERLYAND, L. and MITYUSHEV, V. Generalized clausius-mossotti formula for random composite with circular fibres. J. Stat. Phys., v. 102, 115-145, 2001.

BERLYAND, L. and MITYUSHEV, V. Increase and decrease of the effective conductivity of two phase composites due to polydispesity. J. Stat. Phys., v. 118, 481-509, 2005.

BORM, S.; GRASEDICK, L. and HACKBUSCH, W. Introduction to hierarchical matrices with applications. Engineering Analysis with Boundary Elements, v. 27, 405-422, 2003.

BRANCATI, A.; ALIABADI, M. and BENEDETTI, I. Hierarchical adaptive cross approximation GMRES technique for solution of acoustic problems using the boundary element method. CMES, v. 43, 149-172, 2009.

CARRER, J.; FLEISCHFRESSER, S.; GARCIA, L. and MANSUR, W. Dynamic analysis of Timoshenko beams by the boundary element method. Engineering Analysis with Boundary Elements, v. 37, 1602-1616, 2013.

CLOUGH, R.W. and PENZIEN, J. Dynamics of Structures. Computer \& Structures, Inc., third edition ed., 2003.

CURGUL, S.; J., K.; VLIET, V. and RUTLEDGE, G.C. Molecular dynamics simulation of sizedependent structural and thermal properties of polymer nanofibers. Macromolecules, v. 40, n. 23, 8483-8489, 2007.

URL: http://dx.doi.org/10.1021/ma0714666

DAW, M.S. and BASKES, M.I. Semiempirical, quantum mechanical calculation of hydrogen embrittlement in metals. Physical Review Letters, v. 50, n. 17, 1285-1288, 1983.

DAW, M.S. and BASKES, M.I. Embedded-atom method: derivation and application to impurities, surfaces, and other defects in metals. Physical Review B, v. 29, n. 12, 6443-6453, 1984.

DOMINGUEZ, J. Boundary elements in dynamics. Computational Mechanics Publications, Southampton, Boston, 1993. 
ERICKSEN, J.L. The Cauchy and Born hypotheses for crystals, in: Phase Transformations and Material Instabilities in Solids. New York: Academics, 1984.

FISH, J. and SHEK, K. Multiscale analysis of composite materials and structures. Composites Science and Technology, v. 60, n. 12, 2547 - 2556, 2000.

URL: http://www.sciencedirect.com/science/article/pii/S0266353800000488

FISH, J. and YU, Q. Multiscale damage modelling for composite materials: theory and computational framework. International Journal for Numerical Methods in Engineering, v. 52, n. 1-2, 161-191, 2001.

URL: http://dx.doi.org/10.1002/nme.276

FU, Z. and POPOV, V. The ACA-BEM approach with a binary-key mosaic partitioning for modelling multiple bubble dynamics. Engineering Analysis with Boundary Elements, v. 50, n. 1, 169-179, 2015.

GALVIS, A. and SOLLERO, P. 2D analysis of intergranular dynamic crack propagation in polycrystalline materials a multiscale cohesive zone model and dual reciprocity boundary elements. Computers \& Structures, v. 164, 1-14, 2016.

GALVIS, A.F. and SOLLERO, P. Boundary element analysis of crack problems in polycrystalline materials. Procedia Materials Science, v. 3, 1928 - 1933, 2014.

GAO, X. and PENG, H. Numerical evaluation of arbitrary singular domain integrals based on radial integration method. Engineering Analysis with Boundary Elements, v. 35, n. 3, 587-593, 2011.

GAO, X.; ZHENG, B.; YANG, K. and ZHANG, C. Radial integration BEM for dynamic coupled thermoelastic analysis under thermal shock loading. Computers \& Structures, v. 158, n. 1, 140-147, 2015.

GAO, X.W. Boundary only integral equations in boundary element analysis. In Proceedings of the International Conference on Boundary Element Techniques. 2001. 
GAO, X.W. The radial integration method for evaluation of domain integrals with boundaryonly discretization. Engineering Analysis with Boundary Elements, v. 26, 905 - 916, 2002.

GAUL, L.; KÖGL, M. and WAGNER, M. Boundary Element Methods for Engineers and Scientists. Springer, 2003.

GIEBERMAN, K. Multilevel approximation of boundary integral operators. Computing, v. 67, $183-207,2001$.

GOLBERG, M.A. The numerical evaluation of particular solutions in the BEM - a review. Boundary Element Communications, v. 6, 99 - 106, 1995.

GOSH, A. and CHAUDHURI, P. Computational modeling of fracture in concrete using a meshfree meso-macro-multiscale method. Computational Materials Science, v. 69, 204-215, 2013.

GRASEDYCK, L. Adaptive recompression of H-matrices for BEM. Computing, v. 74, 205$223,2005$.

GRASEDYCK, L. and HACKBUSCH, W. Construction and arithmetics of H-matrices. Computing, v. 70, 295-334, 2003.

GRUNDEMANN, H. A general procedure transferring domain integrals onto boundary integrals in BEM. Engineering Analysis with Boundary Elements, v. 6, n. 4, 214 - 222, 1989.

HACKBUSCH, W. A sparse matrix arithmetic based on h-matrices. part I. Computing, v. 62, 89-108, 1999.

HACKBUSCH, W.; KHOROMSKIJ, B. and KRIEMANN, R. Hierarchical matrices based on weak admissibility criterion. Computing, v. 73, 207-243, 2004.

HACKBUSCH, W. and NOWAK, Z. A sparse H-matrix arithmetic. part II. application to multidimensional problems. Computing, v. 64, 21-47, 2000. 
HAHN, H. and TSAI, S. On the behavior of composite laminates after initial failures. Journal of Composite Materials, v. 8, n. 3, 288-305, 1974.

URL: http://jcm.sagepub.com/content/8/3/288.abstract

HALPIN, J.; KOPF, J.R. and GOLDBERG, W. Time dependent static strength and reliability for composites. Journal of Composite Materials, v. 4, n. 4, 462-474, 1970.

URL: http://jcm.sagepub.com/content/4/4/462.abstract

HALPIN, J.C. Structure - property relations and reliability concepts. Journal of Composite Materials, v. 6, n. 2, 208-231, 1972.

URL: http://jcm.sagepub.com/content/6/2/208.abstract

HALPIN, J.C. and KARDOS, J.L. The halpin-tsai equations: A review. Polymer Engineering and Science, v. 16, 344-352, 1976.

HASSANI, B. A review of homogenization and topology opimization ii - analytical and numerical solution of homogenization equations. Computers \& Structures, v. 69, n. 6, 719-738, December 1998.

HASSANI, B. and HINTON, E. A review of homogenization and topology optimization i homogenization theory for media with periodic structure. Computers \& Structures, v. 69, n. 6, 707-717, 1998a.

HASSANI, B. and HINTON, E. A review of homogenization and topology optimization iii topology optimization using optimality criteria. Computers \& structures, v. 69, n. 6, 739-756, 1998b.

HILL, R. Continuum micro-mechanics of elastoplastic polycrystals. Journal of the Mechanics and Physics of Solids, v. 13, n. 2, 89 - 101, 1965.

URL: http://www.sciencedirect.com/science/article/pii/0022509665900232

HILL, R. On constitutive macro-variables for heterogeneous solids at finite strain. Proc. R. Soc. London, v. 326, 131 - 147, 1972. 
HORSTEMEYER, M. chapter 4: Multiscale Modeling: A review. Springer, 2009.

HOUBOLT, J.C. A recurrence matrix solution for the dynamic response of elastic aircraft. Journal of Aeronautical Sciences, v. 17, 540 - 550, 1950.

HUNTINGTON, E.B. The elastic constants of crystals. New York: Academic Press, 1958.

ISMAIL, A.E.; RUTLEDGE, G.C. and STEPHANOPOULOS, G. Using wavelet transforms for multiresolution materials modeling. Computers \& Chemical Engineering, v. 29, n. 4, 689 - 700, 2005. Control of Multiscale and Distributed Process Systems.

URL: http://www.sciencedirect.com/science/article/pii/S0098135404002777

ISRAELACHVILI, J. Intermolecular \& surface forces. Academic Press, 1992.

JIN, K.; HUANG, Y.; LEE, Y. and HA, S. Distribution of micro stresses and interfacial tractions in unidirectional composites. Journal of Composite Materials, v. 42, 1825-1849, 2008.

JOHNSON, R.A. and OH, D.J. Analytic embedded atom method model for bcc metals. Journal of Materials Research, v. 4, 1195-1201, 1989.

KALAMKAROV, A.L.; ANDRIANOV, I.V. and DANISHEVS'KYY, V.V. Asymptotic homogenization of composite materials and structures. Applied Mechanics Reviews, v. 62, 1-20, 2009.

KANE, J.H. Boundary Element Analysis in Engineering Continuum Mechanics. Prentice Hall, 1994.

KASTNER, M.; MULLER, S. and ULBRICHT, V. XFEM modelling of inelastic material behavior and interface failure in textile-reinforced composites. Procedia Materials Science 2, v. 2, 43-51, 2013.

KELLER, J.B.; RUBENFELD, L.A. and MOLYNEUX, J.E. Extremum principles for slow viscous flows with applications to suspensions. J. Fluid Mech., v. 30, 97-125, 1967. 
KÖGL, M. and GAUL, L. A 3D boundary element method for dynamic analysis of anisotropic elastic solids. Computational Modeling in Engineering \& Sciences, v. 1, n. 4, 27 - 43, 2000a.

KÖGL, M. and GAUL, L. A boundary element method for transient piezoelectric analysis. Engineering Analysis with Boundary Elements, v. 24, 591-598, 2000b.

KÖGL, M. and GAUL, L. Free vibration analysis of anisotropic solids with the boundary element method. Engineering Analysis with Boundary Elements, v. 27, 107-114, 2003.

KOLZLOV, G. Geometrical aspects of averaging. Russ. Math. Surveys, v. 44, 91-144, 1989.

KURZ, S.; RAIN, O. and RJASANOW, S. Fast boundary element methods in computational Electromagnetism. Springer, 2007.

LEE, V. Explicit expression of derivatives of elastic Green's functions for general anisotropic materials. Mech. Res. Comm., v. 30, 241-249, 2003.

LEE, V.G. Derivatives of the three-dimensional Green's function for anisotropic materials. International Journal for Solids and Structures, v. 46, 3471-3479, 2009.

LEKHNITSKII, S.G. Theory of Elasticity of an Anisotropic Elastic Body. Holden-day, 1963.

LEKHNITSKII, S.G. Theory of Elasticity of an Anisotropic Body. Mir, 1981.

LI, S.; ZENG, X.; BO, R.; QIAN, J.; ZHANG, J. and JHA, A.K. An atomistic-based interphase zone model for crystalline solids. Computer Methods in Applied Mechanics and Engineering, v. 223, $87-109,2012$.

LIFSHITZ, I.M. and ROZENZWEIG, L.N. Construction of the green tensor fot the fundamental equation of elasticity theory in the case of unbounded elastic anisotropic medium. Zh. Eksp. Teor. Fiz., v. 17, 783-791, 1947.

LIU, Y. Fast Multipole Boundary Element Method. Cambridge University Press, 2009. 
LOEFFLER, C.F. and MANSUR, W.J. Analysis of time integration schemes for boundary element applications to transient wave propagation problems. In: Boundary Element Techniques: Applications in Stress Analysis and Heat transfer. Southampton, Boston: Computational Mechanics Publications, 1987.

LORENZ, C.D.; STEVENS, M.J. and WOOL, R.P. Fracture behavior of triglyceride-based adhesives. Journal of Polymer Science, Part B: Polymer Physics, v. 1, n. 42, 3333-3343, 2004.

MAITI, A. Multiscale modeling with carbon nanotubes. Microelectron. J., v. 39, 208-221, 2008.

MALLARDO, V.; ALIABADI, M.; BRANCATI, A. and MARANT, V. An accelerated BEM for simulation of noise control in the aircraft cabin. Aerospace Science and Technology, v. 23, n. 1, 418-428, 2012.

MATOUS, K.; KULKARNI, M.G. and GEUBELLE, P.H. Multiscale cohesive failure modeling of heterogeneous adhesives. Journal of the Mechanics and Physics of Solids, v. 56, n. 4, 1511 - 1533, 2008.

URL: http://www.sciencedirect.com/science/article/pii/S0022509607001718

MATSUMOTO, T.; GUZIK, A. and TANAKA, M. A boundary element method for analysis of thermoelastic deformations in materials with temperature dependent properties. International Journal for Numerical Methods in Engineering, v. 64, n. 11, 1432-1458, 2005.

MCCARTNEY, L.N. Physically based damage models for laminated composites. Proc. Instn Mech. Engrs, v. 217, 163-199, 2003.

MEDEIROS, G. and PARTRIDGE, P. The method of fundamental solutions with dual reciprocity for some linear elastic problems in 3D. International Journal of Computational Methods, v. 4, n. 3, 459-474, 2007.

MILAZZO, A.; BENEDETTI, I. and ALIABADI, M. Hierarchical fast BEM for anisotropic time-harmonic 3-D elastodynamics. Computers \& Structures, v. 96, n. 1, 9-24, 2012. 
MOLCHANOV, S.A. Ideas in the theory of random media. Acta Appl. Math., v. 22, 139-282, 1991.

NARDINI, D. and BREBBIA, C.A. A New approach to free vibration analysis using boundary elements. Springer, 1982.

NATALINI, B. and POPOV, V. On the optimal implementation of the boundary element dual reciprocity method-multi-domain approach for 3D problems. Engineering Analysis with Boundary Elements, v. 31, n. 3, 275-287, 2007.

NEWMARK, N.M. A method of computation for structural dynamics. Journal of Engineering Mechanics Division, v. 85, 67 - 94, 1959.

OH, D.J. and JOHNSON, R.A. Simple embedded atom method model for FCC and HCP metals. Journal of Materials Research, v. 3, n. 3, 471-478, 1992.

PALEY, M. and ABOUDI, J. Micromechanical analysis of composites by the generalized cells model. Mechanics of Materials, v. 14, n. 2, 127 - 139, 1992.

URL: http://www.sciencedirect.com/science/article/pii/016766369290010B

PARK, K.C. An improved stiffly stable method for direct integration of nonlinear structural dynamic equations. ASME Journal of Applied Mechanics, v. 42, 464 - 470, 1975.

PARTRIDGE, P.W.; BREBBIA, C.A. and WROBEL, L.C. The Dual Reciprocity Boundary Element Method. Elsevier, 1992.

PERRINS, W.T.; MCKENZIE, D.R. and MCPHEDRAN, R.C. Transport properties of regular array cylinders. Proc. R. Soc. Lond. A, v. 369, 207-225, 1979.

PHAN, P.V.; GRAY, L.J. and KAPLAN, T. On the residue calculus evaluation of the $3 \mathrm{~d}$ anisotropic elastic green's function. Commun. Numer. Methods Eng., v. 20, 335-341, 2004.

QU, S.; LI, S.; CHEN, H. and QU, Z. Radial integration boundary element method for acoustic eigenvalue problems. Engineering Analysis with Boundary Elements, v. 37, 1043-1051, 
2013.

QUIAN, D.; WAGNER, G.J.; LIU, W.K.; YU, M.F. and RUOFF, R.S. Mechanics of carbon nanotubes. Appl. Mech. Rev., v. 55, n. 6, 495-533, 2002.

REN, B. and LI, S. A three-dimensional atomistic-based process zone model simulation of fragmentation in polycrystalline solids. International Journal for Numerical Methods in Engineering, v. 93, 989 - 1014, 2013.

RJASANOW, S. and WEGGLER, L. ACA accelerated high order BEM for maxwell problems. Computational Mechanics, v. 51, n. 4, 431-441, 2013.

RODRÍGUEZ, R.; GALVIS, A.F.; SOLLERO, P. and ALBUQUERQUE, E. Analysis of multiple inclusion potential problems by the adaptive cross approximation method. Computational Modeling in Engineering \& Sciences, v. 96, 259-274, 2013.

RODRÍGUEZ, R.; SOLLERO, P. and ALBUQUERQUE, E. Analysis of anisotropic symmetric plates by the adaptive cross approximation. In Advances in Boundary Element \& Meshless Techniques, pp. 303-310. 2012.

ROKHLIN, H. Rapid solution of integral equation of classical potential theory. Journal of Computational Physics, v. 60, 187-207, 1985.

RUBENFELD, L.A. and KELLER, J.B. Bounds on the elastic moduli of composite media, siam. J. Appl. Math., v. 17, 495-510, 1969.

SAEZ, A. and DOMINGUEZ, J. BEM analysis of wave scattering in transversely isotropic solids. International Journal for Numerical Methods in Engineering, v. 44, 1283-1300, 1999.

SAFAEI, M.; SHEIDAEI, A.; BANIASSADI, M.; AHZI, S.; MASHHADI, M.M. and POURBOGHRAT, F. An interfacial debonding-induced damage model for graphite nanoplatelet polymer composites. Computational Materials Science, v. 96, 191-199, 2015. 
SALES, M.A. and GRAY, L.J. Evaluation of the anisotropic green's function and its derivatives. Comput. Struct., v. 69, 247-254, 1998.

SCHCLAR, N.A. Anisotropic Analysis Using Boundary Elements. Computational Mechanics Publications, 1994.

SHIAH, Y.C. and TAN, C.L. The boundary integral equation for 3D general anisotropic thermoelasticity. Computer Modeling in Engineering \& Sciences, v. 102, n. 6, 425-447, 2014.

SHIAH, Y.C.; TAN, C.L. and LEE, R.F. Internal point solutions for displacements and stresses in 3D anisotropic elastic solids using the boundary element method. CMES, v. 69, 167-197, 2010 .

SHIAH, Y.C.; TAN, C.L. and LEE, V.G. Evaluation of explicit-formfundamental solutions for displacements and stresses in 3d anisotropic elastic solids. CMES, v. 34, 205-226, 2008.

SHIAH, Y.C.; TAN, C.L. and WANG, C.Y. Efficient computation of the green function and its derivatives for three-dimensional anisotropic elasticity in BEM analysis. Engineering Analysis with Boundary Elements, v. 36, 1746-1755, 2012a.

SHIAH, Y.C.; TAN, C.L. and WANG, C.Y. An efficient numerical scheme for the evaluation of the fundamental solution and its derivatives in 3D generally anisotropic elasticity. In Advances In Boundary Element and Meshless Techniques XIII, pp. 190-199. 2012 b.

SODEN, P.D.; HINTON, M.J. and KADDOUR, A.S. Lamina properties, lay-up configurations and loading conditions for a range of fibre-reinforced composite laminates. Composite Science and Technology, v. 58, 1011 - 1022, 1998.

TAN, C.L.; SHIAH, Y.C. and LIN, C.W. Stress analysis of 3D generally anisotropic elastic solids using the boundary element method. CMES, v. 41, 195-214, 2009.

TAN, C.L.; SHIAH, Y.C. and WANG, C.Y. Boundary element elastic stress analysis of 3D generally anisotropic solids using fundamental solutions based on fourier series. International Journal of Solids and Structures, v. 50, 2701-2711, 2013. 
TAVARA, L.; ORTIZ, J.E.; MANTIC, V. and PARIS, R. Unique real-variable eexpression of displacement and traction fundamental solutions covering all transversely isotropic materials for 3D bem. Int. J. Numer. Methods Eng., v. 74, 776-798, 2008.

THEODUROU, D.N. Hierarchical modeling of amorphous polymers. Comp. Phys. Comm., v. 169, 82-88, 2005.

TIMOSHENKO, S. and GOODIER, J. Theory of elasticity. McGraw-Hill, 3rd edition ed., 1970.

TING, T.C.T. Anistropic Elasticity - Theory and Applications. Oxford University Press, 1996.

TING, T.C.T. and LEE, V.G. The three-dimensional elastostatic Green's function for general anisotropic linear elastic solids. J. Mech. Appl. Math., v. 50, 407-426, 1997.

TONON, F.; PAN, E. and AMADEI, B. Green's functions and boundary element method formulation for 3d anisotropic media. Comput. Struct., v. 79, 469-482, 2001.

TORQUATO, S. Random Heterogeneous Materials. Springer, 2002.

TORQUATO, S. and RUBINSHTEIN, J. Improved bounds on the effective conductivity of high contrast suspensions. J. Appl. Phys., v. 69, 7118-7125, 1991.

UGURLU, B. A dual reciprocity boundary element solution method for the free vibration analysis of fluid-coupled kirchhoff plates. Journal of Sound and Vibration, v. 340, n. 1, 190$210,2015$.

USECHE, J. Boundary element analysis of cracked Reissners plates repaired with adhesively bonded composite patches. 2007. PhD Thesis. University of Campinas.

USECHE, J. and HARNISH, C. A boundary element method formulation for modal analysis of doubly curved thick shallows shells. Applied Mathematical Modelling, v. 40, 3591-3600, 2016. 
VENTURINI, W. A study of boundary element method and its application on engineering problems. Professorial thesis, Sao Carlos, University of Sao Paulo, 1988.

VIGUERAS, G.; SKET, F.; SAMANIEGO, C.; WU, L.; NOELS, L.; TJAHJANTO, D.; CASONI, E.; HOUZEAUX, G.; MAKRADI, A.; MOLINA-ALDAREGUIA, J.M.; VAZQUEZ, M. and JERUSALEM, A. An XFEM/CZM implementation for massively parallel simulations of composites fracture. Composite Structures, v. 125, 542-557, 2015.

WANG, C. and ACHENBACH, J. 3D time harmonic elastodynamic green's function for anisotropic solids. Proc. of Royal Society of London, v. A449, 441-458, 1995.

WANG, C. and ACHENBACH, J. Two dimensional time domain BEM for scattering of elastic wave in solids of general anisotropy. International Journal of Solids and Structures, v. 33, 3843-3864, 1996.

WANG, C.Y. and DENDA, M. 3D bem for general anisotropic elasticity. Int. J. Solids Struct., v. 44, 7073-7091, 2007.

WANG, J. Investigating some technical issues on cohesive zone modeling of fracture. Journal of Engineering Materials and Technology, v. 135, 1 - 9, 2013.

WESCOTT, J. Atomistic, mesoscale and finite element simulation of nanofube dispersion in polymers. VDI Berichte, v. 1940, 23-24, 2006.

WILLIAMS, T.O. and TIPPETTS, T.B. Materials damage prognosis. In Proceedings of a Sym. of the Matls. Sci. and Tech. Conf., pp. 95-101. 2004.

WILSON, R. and CRUSE, T. Efficient implementation of anisotropic three dimensional boundary-integral equation stress analysis. International Journal for Numerical Methods in Engineering, v. 12, 1383-1397, 1978.

WOOD, W.L. Practical Time Stepping Schemes. Oxford: Clarendon Press, 1990.

WROBEL, L.C. and BREBBIA, C.A. The dual reciprocity boundary element formulation for 
non-linear diffusion problems. Computer Methods in Applied Mechanics and Engineering, v. 65, n. $2,147-164,1987$.

YAN, F.; MIAO, Y. and CHEUNG, Y. Dual reciprocity hybrid boundary node method for free vibration analysis. Journal of Sound and Vibration, v. 321, n. 3, 1036-1057, 2009.

YANG, D.; SHENG, Y.; YE, J. and TAN, Y. Dynamic simulation of crack initiation and propagation in cross-ply laminates by DEM. Composites Science and Technology, v. 71, 1410$1418,2011$.

YANG, K. and GAO, X. Radial integration BEM for transient heat conduction problems. Engineering Analysis with Boundary Elements, v. 34, n. 6, 557-563, 2010.

YANG, S.; CUI, Z. and QU, J. A coarse-grained model for epoxy molding compound. The Journal of Physical Chemistry B, v. 1, n. 118, 1660-1669, 2014.

YANOVSKY, Y.G. Multiscale modeling of polymer composite properties. International Journal for Multiscale Computational Engineering, v. 3, n. 2, 199-225, 2005.

YAO, W.; YAO, H. and YU, B. Radial integration BEM for solving non-fourier heat conduction problems. Engineering Analysis with Boundary Elements, v. 60, n. 1, 18-26, 2015.

YU, B.; YAO, W.; GAO, X. and ZHANG, S. Radial integration bem for one-phase solidification problems. Engineering Analysis with Boundary Elements, v. 39, n. 1, 36-43, 2014.

YU, Q. and FISH, J. Multiscale asymptotic homogenization for multiphysics problems with multiple spatial and temporal scales: a coupled thermo-viscoelastic example problem. International Journal of Solids and Structures, v. 39, n. 26, 6429 - 6452, 2002.

URL: http://www.sciencedirect.com/science/article/pii/S002076830200255X

YUAN, X.J.; CHEN, N.X.; SHEN, J. and HU, W. Embedded-atom-method interatomic potentials from lattice inversion. Journal of Physics: Condensed Matter, v. 22, n. 37, 375-503, 2010. 
ZENG, X. and LI, S. A multiscale cohesive zone model and simulations of fractures. Comput. Methods Appl. Mech. Eng., v. 199, 547 - 556, 2010.

ZENG, X. and LI, S. Application of a multiscale cohesive zone method to model composite materials. Journal of Multiscale Computational Engineering, v. 10, n. 5, 391 - 405, 2012.

ZHANG, C. Transient elastodynamic antiplane crack analysis of anisotropic solids. International Journal of Solids and Structures, v. 37, 6107-6130, 2000.

ZHEN, J.Q. and BREBBIA, C.A. Boundary element analysis of composite materials. In Proceedings International Conference Computer Aided Design in Composite Material Technology, pp. 475 - 498. 1988. 